\title{
Generic coarse geometry of leaves
}

\author{
Jesús A. Álvarez López \\ Alberto Candel \\ Departamento de Xeometría e Topoloxía, Facultade de Matemáticas, \\ Campus Vida, Universidade de Santiago de Compostela, 15782 Santi- \\ ago de Compostela, Spain \\ E-mail address: jesus.alvarez@usc.es \\ Department of Mathematics, California State University at Northridge, \\ 18111 Nordhoff Street, Northridge, CA 91330, U.S.A. \\ E-mail address: alberto.candel@csun.edu
}


2010 Mathematics Subject Classification. Primary 57R30

Key words and phrases. Foliated space, leaf, holonomy, pseudogroup, orbit, minimal, transitive, metric space, coarse quasi-isometry, coarse quasi-symmetric, growth, growth symmetric, amenable, amenably symmetric, recurrence

Research of the authors supported by Spanish MICINN grants MTM2011-25656 and MTM2008-02640. 


\section{Contents}

Chapter 1. Introduction 1

Chapter 2. Coarse quasi-isometries 11

2.1. Notation, conventions and terminology 11

2.2. Coarse quasi-isometries

2.3. Coarse composites 13

2.4. A coarsely quasi-isometric version of Arzela-Ascoli theorem

2.5. Large scale Lipschitz maps

2.6. Coarse and rough maps 20

Chapter 3. Some classes of metric spaces

3.1. Graphs 25

3.2. Metric spaces of coarse bounded geometry 27

3.3. Coarsely quasi-symmetric metric spaces 29

3.4. Coarsely quasi-convex metric spaces

Chapter 4. Growth of metric spaces 33

4.1. Growth of non-decreasing functions 33

4.2. Growth of metric spaces 34

4.3. Growth symmetry 36

Chapter 5. Amenability of metric spaces 37

5.1. Amenability 37

5.2. Amenable symmetry 40

Chapter 6. Coarse ends 43

6.1. Ends 43

6.2. Coarse connectivity 43

6.3. Coarse ends 45

6.4. Functoriality of the space of coarse ends 49

6.5. Coarse end space of a class of metric spaces 51

Chapter 7. Higson corona and asymptotic dimension

7.1. Compactifications

7.2. Higson compactification

7.3. Asymptotic dimension 61

Chapter 8. Pseudogroups 63

8.1. Pseudogroups 63

8.2. Coarse quasi-isometry type of orbits 64

8.3. A version of local Reeb stability 66 
8.4. Topological dynamics 69

Chapter 9. Generic coarse geometry of orbits

9.1. Coarsely quasi-isometric orbits

9.2. Growth of the orbits 78

9.3. Amenable orbits 83

9.4. Asymptotic dimension of the orbits 85

9.5. Highson corona of the orbits 87

9.6. Measure theoretic versions 91

Chapter 10. Generic coarse geometry of leaves

10.1. Foliated spaces 93

10.2. Saturated sets 95

10.3. Coarse quasi-isometry type of the leaves

10.4. Higson corona of the leaves 100

10.5. Algebraic asymptotic invariants

10.6. Versions with quasi-invariant currents 104

10.7. There is no measure theoretic version of recurrence 106

Chapter 11. Examples and open problems 109

11.1. Foliated spaces defined by suspensions

11.2. Foliated spaces defined by locally free actions of Lie groups

11.3. Inverse limits of covering spaces 110

11.4. Bounded geometry and leaves 111

11.5. Graph spaces

11.6. Case of Cayley graphs 1114

11.7. Construction of limit-aperiodic functions 116

11.8. Graph matchbox manifolds 117

11.9. Concrete examples of graph matchbox manifolds 119

11.10. Foliations of codimension one 123

11.11. Open problems

Bibliography 133 
Abstract. A compact Polish foliated space is considered. Part of this work studies coarsely quasi-isometric invariants of leaves in some residual saturated subset when the foliated space is transitive. In fact, we also use "equi-" versions of this kind of invariants, which means that the definition is satisfied with the same constants by some given set of leaves. For instance, the following properties are proved.

Either all dense leaves without holonomy are equi-coarsely quasi-isometric to each other, or else there exist residually many dense leaves without holonomy such that each of them is coarsely quasi-isometric to meagerly many leaves. Assuming that the foliated space is minimal, the first of the above alternatives holds if and if the leaves without holonomy satisfy a condition called coarse quasi-symmetry.

A similar dichotomy holds for the growth type of the leaves, as well as an analogous characterization of the first alternative in the minimal case, involving a property called growth symmetry. Moreover some classes of growth are shared, either by residually many leaves, or by meagerly many leaves.

If some leaf without holonomy is amenable, then all dense leaves without holonomy are equi-amenable, and, in the minimal case, they satisfy a property called amenable symmetry.

Residually many leaves have the same asymptotic dimension.

If the foliated space is minimal, then any pair of nonempty open sets in the Higson coronas of the leaves with holonomy contain homeomorphic nonempty open subsets.

Another part studies limit sets of leaves at points in the coronas of their compactifications, defined like their usual limit sets at their ends. These sets are nonempty and compact, but they may not be saturated. The following properties are shown.

The limit sets are saturated for compactifications less or equal than the Higson compactification of the leaves. This establishes a relation between the coarse geometry of the leaves and the structure of closed saturated sets.

For any given leaf, its limit set at every point in its Higson corona is the whole space if and only if the foliated space is minimal.

For some dense open subset of points in the Higson corona of any leaf, the corresponding limit sets are minimal sets. 

Contents 



\section{CHAPTER 1}

\section{Introduction}

The work presented in this monograph is about the metric and topological properties of Riemannian manifolds that arise as leaves of compact foliated spaces, and more generally about the metric and topological porperties of orbits of pseudogroups compactly generated pseudogroups of homeomorphisms of topological spaces.

Since the inception of foliation theory as a proper mathematical subject of research, with Reeb and Ehreshman and Haefliger, the problem of which manifolds can be leaves has been one of fundamental interest and motive of research, even if not explicitly stated as such. In its purest essence, a foliation of a manifold is a decomposition of the manifold into equidimensional submanifolds, the leaves, and it is a natural question to understand how they are assembled, and how the nature of the ambient space affects their structure. In fact, it could, in retrospect, be said that the problem appeared even before the first foliation of the three dimensional sphere was explicitly known: because of algebraic topological reasons, a two dimensional leaf of a foliation of $S^{3}$ must be a torus. Reeb constructed the first foliation of $S^{3}$, and what it has since called a Reeb component: a foliated solid torus with one compact leaf and a bundle of planes each having linear, rather than quadratic, area growth (later Novikov proved that any foliation of $S^{3}$ must contain at least one Reeb component).

The problems thus suggested by the structure of the Reeb foliation, and by the structure of the topology of flows (Poincaré-Bendixon theory, the Denjoy flow and the Cherry flow of the torus) took a definite position in mathematical research in the early 1970's with the work of many on many topics, for example: growth and invariant measures (Hirsch, Thurston, Plante, Mossu-Pelletier, Anosov, RuelleSullivan), realizability of manifolds as leaves (Sullivan, Sondow, Cantwell-Conlon, Raymond), non-leaves (Ghys, Inaba et al.), limit sets of leaves (Duminy). Sullivan posed the explicit problem in [96]: Problem 8. What do 2-dimensional leaves in $S^{3}$ look like?

It is not our intention to give a complete historical account of these developments because they are well surveyed in the paper of Hurder [68, which also introduced to the study of foliation theory many of the concepts studied in the present work.

We would also like to make a explitic mention of the paper of D. Cass [31] who gave the first published results relating the recurrence properties of leaves of foliations with their quasi-isometry types, and who quoted an unpublished result of Gromov that we have developed in [7].

Paraphrasing a referee, the work to be presented can be considered as the complete development of the ideas behind Cass work, the continuation of our work on 
the descriptive set theory aspects of foliations $[\mathbf{8}$ and $[\mathbf{9}$, and the further applications of these ideas to the properties of the coarse geometry of open complete manifolds which arise as leaves of foliations of compact manifolds.

We consider (compact) Polish foliated spaces. The original question that motivated our work, here and elsewhere, was not the original Problem 8 of Sullivan cited above: what do the leaves of a foliation look like?, but rather: when do all leaves of a foliated space look alike? (the quantifier "all leaves" is taken in the relaxed sense of category theory or measure theory). By "look alike" we not only mean the strongest possibility of "being quasi-isometric," but also that possibility of sharing a common quasi-isometry invariant, like having the same growth type, or the same end-point compactification, for example. In fact, we also use "equi-" versions of this kind of invariants, which means that the definition is satisfied with the same constants by some given set of leaves. For instance, the following properties are proved.

We prove the following complete characterization of the when and how all leaves "look alike." Either all dense leaves without holonomy are equi-coarsely quasi-isometric to each other, or else there exist residually many dense leaves without holonomy such that each of them is coarsely quasi-isometric to meagerly many leaves. Assuming that the foliated space is minimal, the first of the above alternatives holds if and if the leaves without holonomy satisfy a condition called coarse quasi-symmetry.

We also show that a similar dichotomy holds for the growth type of the leaves, as well as an analogous characterization of the first alternative in the minimal case, involving a property called growth symmetry. Moreover some classes of growth are shared, either by residually many leaves, or by meagerly many leaves.

Other quasi-isometry invariants that we study are amenability: if some leaf without holonomy is amenable, then all dense leaves without holonomy are equiamenable (in the minimal case, they satisfy a property called amenable symmetry), and asymptotic dimension: residually many leaves have the same asymptotic dimension.

Limit sets of leaves of foliated spaces have always been a fundamental topic of research (originating with the Poincaré-Bendixon theory for planar differential equations). The limits sets that we study here arise from points in the coronas of compactifications of leaves. Tradicionally the compactification studied has been the end-point compactification, but from the point of view of quasi-isometry, the Higson compactification is more relevant (as we have shown elsewhere, this compactification characterizes quasi-isometric spaces).

First we show that, in certain sense, all leaves look alike at infinity: if the foliated space is minimal, then any pair of nonempty open sets in the Higson coronas of the leaves with holonomy contain homeomorphic nonempty open subsets.

After that we study limit sets of leaves at points in the coronas of their compactifications, defined like their usual limit sets at their ends. These sets are nonempty and compact, but they may not be saturated (union of leaves). The following properties are shown: (a) The limit sets are saturated for compactifications less or equal than the Higson compactification of the leaves (this establishes a relation between the coarse geometry of the leaves and the structure of closed saturated sets); (b) for any given leaf, its limit set at every point in its Higson corona is the whole space if and only if the foliated space is minimal; and (c) for some dense open subset of 
points in the Higson corona of any leaf, the corresponding limit sets are minimal sets.

To be more precise, let $(X, \mathcal{F})$ be a compact Polish foliated space; i.e., this foliated space is compact, Hausdorff and second countable. A regular foliated atlas, $\mathcal{U}$, for $(X, \mathcal{F})$ induces a coarse metric, $d_{\mathcal{U}}$, on each leaf $L$ [68: for $x, y \in L, d_{\mathcal{U}}(x, y)$ is the minimum number of $\mathcal{U}$-plaques whose union is connected and contains $x$ and $y$. A metric $d_{\mathcal{U}}^{*}$ on $L$ can be obtained by modifying $d_{\mathcal{U}}$ on the diagonal of $L \times L$, where $d_{\mathcal{U}}^{*}$ is declared to be zero. By the compactness of $X$ and the regularity of $\mathcal{U}$, it follows that the coarse quasi-isometry type of the leaves with $d_{\mathcal{U}}^{*}$ (in the sense of Gromov [55) is independent of the choice $\mathcal{U}$. In this way, the leaves are equipped with a coarse quasi-isometry type (of metrics) determined by $(X, \mathcal{F})$. The leaves of $(X, \mathcal{F})$ belong to a class of metric spaces for which the coarse quasi-isometry type equals the coarse type [85, 86. Thus terms like "coarse equivalence" and "coarse type" could be used as well in our statements. Moreover Roe [86 extended to coarse spaces many of the quasi-isometric invariants and properties we use (bounded geometry, growth, amenability, Higson corona and asymptotic dimension).

Let $(X, \mathcal{F})$ be leafwise differentiable of class $C^{3}\left(C^{k}\right.$ smoothness is defined in 23 ), and $g$ be a $C^{2}$ leafwise Riemannian metric on $X$. Then the coarse quasiisometry type of the leaves is also represented by the Riemannian distance induced on them by $g$. The restrictions of $g$ also define a differentable quasi-isometry type of the leaves, which depends only on $(X, \mathcal{F})$.

The determination of the coarse quasi-isometry type of the leaves of a foliated space obtained in the present work is in fact slightly stronger than stated above. A regular foliated atlas $\mathcal{U}$ for $(X, \mathcal{F})$ determines a set of metric spaces $\left\{\left(L, d_{\mathcal{U}}^{*}\right)\right\}$, where $\{L\}$ is the set of leaves of $(X, \mathcal{F})$. If $\mathcal{U}$ and $\mathcal{V}$ are regular foliated atlases for $(X, \mathcal{F})$, then the set of metric spaces $\left\{\left(L, d_{\mathcal{U}}^{*}\right)\right\}$ is equi-coarsely quasi-isometric to the set of metric spaces $\left\{\left(L, d_{\mathcal{V}}^{*}\right)\right\}$ in the sense that all the coarse quasi-isometries between each pair of metric spaces $\left(L, d_{\mathcal{U}}^{*}\right)$ and $\left(L, d_{\mathcal{V}}^{*}\right)$ share common distortion constants (a coarsely quasi-isometric version of equicontinuity). This kind of terminology will be used with several concepts of metric spaces, with a similar meaning, when their definition involves constants.

The first goal of this work is to study the coarse quasi-isometry properties of the generic leaf of $(X, \mathcal{F})$, that is, to study what quasi-isometry properties are generic in the topological sense that there is a residual saturated subset of the foliated space all whose leaves share that quasi-isometry property. Thus we will focus on the generic coarse properties of the leaves. For the existence of interesting generic properties of the leaves, it will be often required that the foliated space be transitive (some leaf is dense) or even minimal (all leaves are dense); these conditions, transitivity and minimality, are the topological counterparts of ergodicity. For example, the closure of any leaf of $(X, \mathcal{F})$ is a transitive foliated subspace, and any minimal subset of $(X, \mathcal{F})$ is a minimal foliated space. The condition on $X$ to be compact is also useful because it determines the quasi-isometric types of the leaves.

This part may be thus be placed in the area of Ghys [50, and Cantwell and Conlon 28, who have studied the topology of the generic leaf, where "generic" was used in a measure theoretic sense by Ghys, and in a topological sense by Cantwell and Conlon. Indeed, it was Ghys who raised the problem of studying the quasiisometry type of the generic leaf. 
Recall that a set in a topological space is said to be residual if it contains a countable intersection of open dense subsets. The complements of residual sets are called meager. The Borel sets (respectively, Baire sets) are the elements of the the smallest $\sigma$-algebra containing all open sets (respectively, all open sets and all meager sets). Being "generic" in the topological sense refers to a property that holds on a residual set.

For a Polish foliated space $(X, \mathcal{F})$, the following notation will be standing for the rest of this work. The leaf of $(X, \mathcal{F})$ that contains $x \in X$ is denoted by $L_{x}$. The subset of $X$ consisting of the union of all leaves that have no holonomy is denoted by $X_{0}$, and the subset of $X$ consisting of all the leaves that are dense and have no holonomy is denoted by $X_{0, \mathrm{~d}}$.

Hector 61 and Epstein-Millet-Tischler [42] have proved that that $X_{0}$ is a residual subset of $X$, and that, if $(X, \mathcal{F})$ is transitive, then $X_{0, \mathrm{~d}}$ is also residual in $X$. This suggests that, when $(X, \mathcal{F})$ is transitive, residually many leaves might have other properties, besides having no holonomy and being dense. Concerning quasiisometric invariants, we often find out that there is a dichotomy: either residually many leaves share a common value of the invariant, or else every possible value of the invariant is shared by meagerly many leaves. Of course, only the first of these alternatives can happen if the invariant has a countable number of possible values. We also consider relations in $X$ defined by being in leaves with a common value of an invariant, and show that these relations are Borel in $X_{0} \times X_{0}$ and Baire in $X \times X$.

In most of the results of this work, the generic leaf is a leaf in $X_{0}$ or in $X_{0, \mathrm{~d}}$. The reason is that an essential tool of the proof is the local Reeb stability theorem, which involves the holonomy of the leaves.

The main theorems are stated presently.

Theorem 1.1. Let $(X, \mathcal{F})$ be a compact Polish foliated space. The equivalence relation " $x \sim y$ if and only if the leaf $L_{x}$ is coarsely quasi-isometric to the leaf $L_{y}$ " has a Borel relation set in $X_{0} \times X_{0}$, and has a Baire relation set in $X \times X$; in particular, it has Borel equivalence classes in $X_{0}$, and Baire equivalence classes in $X$.

TheOrem 1.2. Let $(X, \mathcal{F})$ be a transitive compact Polish foliated space. Then the following dichotomy holds:

(i) Either all leaves in $X_{0, \mathrm{~d}}$ are equi-coarsely quasi-isometric to each other; or else

(ii) every leaf in $X$ is coarsely quasi-isometric to meagerly many leaves; in particular, in this case, there are uncountably many coarse quasi-isometry types of leaves in $X_{0, \mathrm{~d}}$.

In Theorem 1.2 the alternative (ii) means that there exists a generic quasiisometry type of leaves. This holds for instance in the case of equicontinuous foliated spaces under some mild conditions [10.

The next theorem characterizes the alternative (i) of Theorem 1.2 by using a property of metric spaces called coarse quasi-symmetry. A metric space is coarsely quasi-symmetric if it admits a set of equi-coarsely quasi-isometric transformations that is transitive in the sense that, for every pair of points of the metric space, there is a coarse quasi-isometry in that set mapping one point onto the other. (Equi-) coarse quasi-symmetry is invariant by (equi-) coarse quasi isometries. 
THEOREM 1.3. The following properties hold for a compact Polish foliated space $(X, \mathcal{F})$ :

(i) Suppose that $(X, \mathcal{F})$ is transitive. If one leaf is coarsely quasi-symmetric, then the alternative (i) of Theorem 1.2 holds.

(ii) Suppose that $(X, \mathcal{F})$ is minimal. If the alternative (i) of Theorem 1.2 holds, then all leaves in $X_{0}$ are equi-coarsely quasi-symmetric.

Roe [84, Proposition 2.25] notes that the number of ends is a coarse invariant for some class of metric spaces, which includes the leaves of $X$. Furthermore Bridson and Haefliger have proved that the end space is a coarsely quasi-isometric invariant of proper geodesic spaces [21, Proposition 8.29]. Indeed the end space is a coarse invariant of each leaf; this is proved by introducing the coarse end space of any metric space, and showing that it equals the usual end space under certain conditions.

The space of ends of the generic leaf plays an important role in the indicated studies of its topology; in particular, a slight simplification of [28, Theorem A] states that, if $(X, \mathcal{F})$ is minimal, then residually many leaves without holonomy have zero, one, two or a Cantor space of ends, simultaneously. Since the end space is a coarse invariant, we directly get the following slight improvement of that result when alternative (i) of Theorem 1.2 holds.

Corollary 1.4. Suppose that $(X, \mathcal{F})$ is minimal and satisfies the alternative (i) of Theorem 1.2. Then all leaves in $X_{0}$ have zero, one, two or a Cantor space of ends, simultaneously.

Another proof of Corollary 1.4 is also given, showing that, under certain mild conditions, any coarsely quasi-symmetric metric space has zero, one, two or a Cantor space of coarse ends (Theorem 6.39).

Corollary 1.4 is useful to give examples of minimal compact Polish foliated spaces satisfying the alternative (iii) of Theorem 1.2. For instance, this alternative is satisfied by the foliated space of Ghys-Kenyon [51] because it has a leaf without holonomy with four ends. Lozano 75 gives variations of the example of GhysKenyon, producing minimal foliated spaces with leaves without holonomy that have any finite number $\geq 3$ of ends; they also must satisfy alternative (ii) of Theorem 1.2 by Corollary 1.4 .

Blanc [19, Thèoreme 1] has shown that, if $\mathcal{F}$ is minimal and residually many leaves have two ends, then all leaves without holonomy are coarsely quasi-isometric to $\mathbb{Z}$, and the leaves with holonomy are coarsely quasi-isometric to $\mathbb{N}$ and have holonomy group $\mathbb{Z} / 2 \mathbb{Z}$. This completely describes the coarse quasi-isometry type of the leaves in the case with two ends of Corollary 1.4. In particular, it follows that $\mathcal{F}$ satisfies alternative (ii) of Theorem 1.2 if it is minimal and has a leaf with two ends that is not coarsely quasi-isometric to $\mathbb{Z}$. For instance, the Ghys-Kenyon foliated space also contains leaves with two ends that are not quasi-isometric to $\mathbb{Z}$ 1 .

Observe that Theorems $1.1,1.3$ and Corollary 1.4 cannot be directly extended to the leaves with finite holonomy groups by the theorem of Blanc quoted above. In that case, the leaves with holonomy are not coarsely quasi-isometric to the leaves with holonomy, and they are not coarsely quasi-symmetric. However they have a common coarse quasi-isometric type, which could be true with more generality. 
One of the classic coarsely quasi-isometric invariants of a leaf $L$ of a compact Polish foliated space $(X, \mathcal{F})$ is its growth type. It can be defined as the growth type of the function $r \mapsto v_{\mathcal{U}}(x, r)(r>0)$, for any regular foliated atlas $\mathcal{U}$ and $x \in L$, where $v_{\mathcal{U}}(x, r)$ is the number of plaques of $\mathcal{U}$ that meet the $d_{\mathcal{U}}^{*}$-ball of center $x$ and radius $r$-this growth type is independent of $\mathcal{U}$ and $x$. If the functions $r \mapsto v_{\mathcal{U}}(x, r)$ $(x \in L)$ have equi-equivalent growth, then $L$ is called growth symmetric. If $(X, \mathcal{F})$ is $C^{3}$ and $g$ is a $C^{2}$ leafwise Riemannian metric on $X$, then the growth type of $L$ equals the usual growth type of the connected Riemannian manifold $L$ with the restriction of $g$.

Block and Weinberger 20] introduced the class of metric spaces of coarse bounded geometry, and defined the growth type for any metric space in this class; it agrees with the above definition for leaves of $\mathcal{F}$, which are included in this class. Growth symmetry can be also defined with this generality. (Equi-) growth type and (equi-) growth symmetry are invariant by (equi-) coarse quasi-isometries.

TheOrem 1.5. Let $(X, \mathcal{F})$ be a compact Polish foliated space. The equivalence relation " $x \sim y$ if and only if $L_{x}$ has the same growth type as $L_{y}$ " has a Borel relation set in $X_{0} \times X_{0}$, and has a Baire relation set in $X \times X$; in particular, it has Borel equivalence classes in $X_{0}$, and Baire equivalence classes in $X$.

TheOrem 1.6. Let $(X, \mathcal{F})$ be a transitive compact Polish foliated space. Then the following dichotomy holds:

(i) Either all leaves in $X_{0, \mathrm{~d}}$ have equi-equivalent growth; or else

(ii) the growth type of each leaf in $X$ is comparable with the growth type of meagerly many leaves; in particular, in this second case, there are uncountably many growth types of leaves in $X_{0, \mathrm{~d}}$.

The alternative (i) of Theorem 1.6 is the case where there is a generic growth type.

TheOrem 1.7. Let $(X, \mathcal{F})$ be a compact Polish foliated space. The following properties hold:

(i) Suppose that $(X, \mathcal{F})$ is transitive. If there is a growth symmetric leaf in $X_{0, \mathrm{~d}}$, then the alternative (i) of Theorem 1.6 holds.

(ii) Suppose that $(X, \mathcal{F})$ is minimal. If the alternative (i) of Theorem 1.6 holds, then all leaves in $X_{0}$ are equi-growth symmetric.

Theorem 1.8. Suppose that $(X, \mathcal{F})$ is a transitive compact Polish foliated space. Then there are $a_{1}, a_{3} \in[1, \infty], a_{2}, a_{4} \in[0, \infty)$ and $p \geq 1$ such that

$$
\begin{array}{cc}
\limsup _{r \rightarrow \infty} \frac{\log v(x, r)}{\log r}=a_{1}, & a_{2} \leq \liminf _{r \rightarrow \infty} \frac{\log v(x, r)}{r} \leq p a_{2}, \\
\liminf _{r \rightarrow \infty} \frac{\log v(x, r)}{\log r}=a_{3}, & \limsup _{r \rightarrow \infty} \frac{\log v(x, r)}{r}=a_{4}
\end{array}
$$

for residually many points $x$ in $X$. Moreover

$$
\liminf _{r \rightarrow \infty} \frac{\log v(x, r)}{\log r} \geq a_{3}, \quad \limsup _{r \rightarrow \infty} \frac{\log v(x, r)}{r} \leq a_{4}
$$

for all $x \in X_{0, \mathrm{~d}}$.

The conditions used in the following result are defined by requiring that the superior and inferior limits used in Theorem 1.8 be $<\infty,>0$ or $\leq 0$ (see Sections 4.1 
and 4.2. All of those terms are standard except pseudo-quasi-polynomial, which is introduced here. It is well known that the growth type of all non-compact leaves of $\mathcal{F}$ is at least linear and at most exponential.

Corollary 1.9. Let $(X, \mathcal{F})$ be a transitive compact Polish foliated space. Then the following sets are either meager or residual in $X$ :

(i) the union of leaves in $X_{0}$ with polynomial growth;

(ii) the union of leaves in $X_{0}$ with exponential growth;

(iii) the union of leaves in $X_{0}$ with quasi-polynomial growth;

(iv) the union of leaves in $X_{0}$ with quasi-exponential growth; and

$(v)$ the union of leaves in $X_{0}$ with pseudo-quasi-polynomial growth.

Moreover,

(a) if the set (iii) is residual in $X$, then it contains $X_{0, \mathrm{~d}}$; and,

(b) if one of the sets (iv) or (v) is meager in $X$, then it does not meet $X_{0, \mathrm{~d}}$.

Hector 62 constructed a remarkable example of a $C^{\infty}$ foliation $\mathcal{F}$ of codimension one on $X=M \times S^{1}$ (where $M$ is the closed oriented surface of genus two), which is transverse to the factor $S^{1}$, satisfying the following properties:

- For each integer $n \geq 0$, there is exactly one proper leaf of exactly polynomial growth of degree $n$. Every other leaf has non-polynomial growth and is dense.

- Each non-polynomial growth class of leaves has the cardinality of the continuum.

- It has one growth class of leaves with exponential growth.

- The set of growth classes which are non-polynomial but quasi-polynomial (respectively, non-quasi-polynomial but non-exponential) has the cardinality of the continuum.

It will be shown that this example satisfies the alternative (ii) of Theorem 1.6 . In fact, the following stronger property will be proved. In Hector's example, the relation of being in leaves with the same growth type is generically ergodic with respect to the isomorphism relation on countable models (Theorem 11.6). The proof uses our work [9] on turbulent relations. This suggests that, in the alternatives Theorem 1.2-(ii) and Theorem 1.6-(ii), it might be true that the relations involved are turbulent, and therefore generically ergodic with respect to the isomorphism relation on countable models (Problem 3).

Cantwell and Conlon 26 have proved that there is a set $G$ of growth types, containing a continuum of distinct quasi-polynomial but non-polynomial types and a continuum of distinct non-exponential but non-quasipolynomial types, together with the exponential type, such that, for any closed $C^{\infty}$ 3-manifold $M$ and $\gamma \in G$, there is a $C^{\infty}$ foliation $\mathcal{F}$ on $M$ with a local minimal set $U$ of locally dense type so that:

- $\bar{U} \backslash U$ is a finite union of totally proper leaves;

- the leaves of $\mathcal{F}$ in $U$ have trivial holonomy and are diffeomorphic one another; and

- each leaf of $\left.\mathcal{F}\right|_{U}$ has growth type $\gamma$.

Then the foliated space $X=\bar{U}$, with the restriction of $\mathcal{F}$, satisfies the alternative (i) of Theorem 1.6 because $X_{0}=X_{0, d} \supset U$ and all leaves have the same growth type on $U$. It also satisfies the alternative (i) of Theorem 1.2 (Section 11.10.5.1). 
Let $\mathcal{U}$ be a regular foliated atlas for $(X, \mathcal{F})$, and let $S$ be a set of $\mathcal{U}$-plaques in the same leaf $L$. The boundary $\partial S$ is the set of $\mathcal{U}$-plaques in $L$ that meet $\mathcal{U}$-plaques in $S$ and outside $S$. It is said that $L$ is $\mathcal{U}$-Følner when there is a sequence of finite sets $S_{n}$ of $\mathcal{U}$-plaques such that $\left|S_{n}\right| /\left|\partial S_{n}\right| \rightarrow 0$ as $n \rightarrow \infty$, where $\left|S_{n}\right|$ is the cardinal of $S_{n}$. This condition is independent of the choice of $\mathcal{U}$, and $L$ is called amenable when it is satisfied. If $(X, \mathcal{F})$ is $C^{3}$ and $g$ is a $C^{2}$ leafwise Riemannian metric on $(X, \mathcal{F})$, then a leaf $L$ is amenable if and only if it is Følner as Riemannian manifold with the restriction of $g$. Amenable symmetry is also introduced for a leaf $L$; roughly speaking, it means that the Følner condition is satisfied uniformly close to any point of $L$, and with the same rate of convergence to zero; the precise statement of this definition is indeed very involved. We will also use a property stronger than equi-amenable symmetry called joint amenable symmetry.

The concept of amenability was extended to arbitrary metric spaces of coarse bounded geometry by Block and Weinberger [20. All of the above variants of amenability can be also defined in this setting, becoming (equi-) coarsely quasiisometric invariants.

Theorem 1.10. Let $(X, \mathcal{F})$ be a compact Polish foliated space. The following properties hold:

(i) If $(X, \mathcal{F})$ is transitive and some leaf in $X_{0}$ is amenable, then all leaves in $X_{0, \mathrm{~d}}$ are equi-amenable.

(ii) If $(X, \mathcal{F})$ is minimal and some leaf in $X_{0}$ is amenable, then all leaves in $X_{0}$ are jointly amenably symmetric.

A foliated space is called amenable when it has an invariant mean on the holonomy pseudogroup, which is not equivalent to amenability (or Følner property) of the leaves [70, 3]. We remark that the concept of amenable foliated space is not used in Theorem 1.10 we only use amenability of the leaves, and some "uniform" versions of amenability of the leaves.

Another coarse invariant of a metric space $M$ is its Higson corona $\nu M$. When $M$ is proper, it is the corona of the Higson compactification $M^{\nu}$, which is defined by applying the Gelfand-Naimark theorem to the bounded continuous functions on $M$ whose variation vanish at infinity. The Higson corona plays an important role in coarse geometry $\left[\mathbf{8 4},\left[\mathbf{8 6}\right.\right.$; indeed, a weak version of the germ of $\nu M$ in $M^{\nu}$ contains all coarse information of $M$ (Proposition 7.4 see also 11]). The semi weak homogeneity used in the following theorem means that, given any pair of nonempty open sets, there is a non-empty open subset of one of them homeomorphic to some open subset of the other one - this is weaker than weak homogeneity [4], which means that, for all points $x$ and $y$, there is a pointed homeomorphism $(U, x) \rightarrow(V, y)$ for some open neighborhoods, $U$ of $x$ and $V$ of $y$.

Theorem 1.11. Let $(X, \mathcal{F})$ be a compact Polish foliated space. If $\mathcal{F}$ is minimal, then the space $\bigsqcup_{L} \nu L$, with $L$ running in the set of all leaves in $X_{0}$, is semi weakly homogeneous.

A numerical coarsely quasi-isometric invariant of metric spaces is their asymptotic dimension, introduced by Gromov [55. It can be defined by using covers by uniformly bounded open sets, in a way dual to the definition of Lebesgue covering dimension (by coarsening the covers instead of refining them). The relevance of this invariant of a metric space is illustrated by a theorem of Dranishnikov, Keesling and Uspenkij $[\mathbf{3 8}$, stating that $\operatorname{dim} \nu M \leq \operatorname{asdim} M$ for all proper metric 
space $M$, which is complemented by another theorem of Dranishnikov [37, stating that $\operatorname{dim} \nu M=\operatorname{asdim} M$ if $\operatorname{asdim} M<\infty$. Moreover Yu 99 related the asymptotic dimension to the Novikov conjecture.

Theorem 1.12. Let $(X, \mathcal{F})$ be a transitive compact Polish foliated space. Then residually many leaves have de same asymptotic dimension.

The following theorem is a coarsely quasi-isometric version of the "Proposition fondamentale" of Ghys [50, p. 402], using generic leaves in a topological sense.

Theorem 1.13. Suppose that $(X, \mathcal{F})$ is a minimal compact Polish foliated space, and let $B$ be a Baire subset of $X$. Then:

(i) either the $\mathcal{F}$-saturation of $B$ is meager; or else

(ii) the intersections of residually many leaves with $B$ are equi-nets in those leaves.

Due to the relevance of Ghys' result in the description of the topology of the generic leaf, we expect that this theorem will be relevant to describe the differentiable quasi-isometry type of generic leaves. We will give a counterexample showing that the measure theoretic version of Theorem 1.13 is false. Thus there may be more difficulties to study the measure theoretic generic differentiable quasi-isometric type of leaves. However weaker measure theoretic versions of Theorems 1.2 1.6 and 1.8 will be proved, involving an ergodic harmonic measure [47] so that $X \backslash X_{0}$ has measure zero.

Another goal of this work is to study the limit sets $\lim _{\mathbf{e}} L$ of a leaf $L$ at a point $\mathbf{e}$ in the corona of any compactification of $L$, which is a straightforward generalization of the usual limit set of $L$, or its e-limit for any end $\mathbf{e}$ of $L$. For a general compactification of $L$, the corresponding limit sets of $L$ are closed in $X$ and nonempty, but they may not be $\mathcal{F}$-saturated. However we will mainly consider the Higson compactification $L^{\nu}$, or compactifications $\bar{L} \leq L^{\nu}$; i.e., $\operatorname{id}_{L}$ has a continuous extension $L^{\nu} \rightarrow \bar{L}$. Then the following theorem gives a bridge between the coarse geometry of the leaves and the structure of closed saturated subsets.

Theorem 1.14. Let $(X, \mathcal{F})$ be a compact Polish foliated space. Let $\bar{L}$ be a compactification of a leaf $L$, with corona $\partial L$. If $\bar{L} \leq L^{\nu}$, then $\lim _{\mathbf{e}} L$ is $\mathcal{F}$-saturated for all $\mathbf{e} \in \partial L$.

As defined by Cantwell and Conlon [28, a leaf $L$ of $\mathcal{F}$ is totally recurrent if $\lim _{\mathbf{e}}=X$ for all end $\mathbf{e}$ of $L$. They showed that the set of totally recurrent leaves of $\mathcal{F}$, if non-empty, is residual. Correspondingly, using the Higson corona, $L$ is said to be Higson recurrent if $\lim _{\mathbf{e}} L=X$ for all $\mathbf{e} \in \nu L$. Higson recurrence behaves in the following manner.

Theorem 1.15. Let $(X, \mathcal{F})$ be a compact Polish foliated space. A leaf is Higson recurrent if and only if $\mathcal{F}$ is minimal.

For each $\mathcal{F}$-minimal set $Y$ and every leaf $L$, let $\nu_{Y} L=\left\{\mathbf{e} \in \nu L \mid \lim _{\mathbf{e}} L=Y\right\}$.

Theorem 1.16. Let $(X, \mathcal{F})$ be a compact Polish foliated space. For any leaf $L$, the space $\cup_{Y} \operatorname{Int}_{\nu L}\left(\nu_{Y} L\right)$, where $Y$ runs in the family of $\mathcal{F}$-minimal sets, is dense in $\nu L$.

The proofs of the main theorems of this work will be carried out in the context of the holonomy pseudogroup of $(X, \mathcal{F})$. For a regular foliated atlas $\mathcal{U}$ for $(X, \mathcal{F})$, the set $E$ of the transverse components of the changes of coordinates generate a 
pseudogroup $\mathcal{H}$ on a space $Z$, which is called a representative of the holonomy pseudogroup of $(X, \mathcal{F})$. In turn, $E$ defines a metric $d_{E}$ on the $\mathcal{H}$-orbits, by setting $d_{E}(x, y)$ to be the smallest number of elements of $E$ whose composition is defined at $x$ and maps $x$ to $y$. Then the $\mathcal{F}$-leaves with $d_{\mathcal{U}}^{*}$ are coarsely quasi-isometric to the $\mathcal{H}$-orbits with $d_{E}$. In this way, our main theorems are easy consequences of their versions for pseudogroups. Most of the work is devoted to prove those pseudogroup versions, as well as to develop the needed tools about metric spaces.

In the case of Theorem 1.13, an alternative direct proof is also given because it is very short and conceptually interesting. Also, this second proof is representative of the type of direct proofs that could be given for other results. However the pseudogroup versions of these theorems have their own interest; for instance, they can be directly applied to orbits of finitely generated group actions on compact Polish spaces.

The structure of this work is the following. Chapters 277 recall the needed concepts and results about coarse quasi-isometric invarints, and gives new concepts and results that will be required in the subsequent chapters. Chapter 8 contains preliminaries about pseudogroups, including the pseudogroup version of Theorem 1.13 and a quasi-isometric version of the Reeb local stability for pseudogroups. Chapter 9 contains proofs of the pseudogroup versions of all other main theorems. In Chapter 10, the needed preliminaries on foliated spaces are recalled, and the main results are obtained from their pseudogroup versions. It also includes a section with the measure theoretic versions of Theorems 1.2, 1.6 and 1.8, and Corollary 1.9, and a section showing that the measure theoretic version of Theorem 1.13 fails. Chapter 11 contains a section devoted to examples, and another section with open problems. 


\section{CHAPTER 2}

\section{Coarse quasi-isometries}

In Chapters 2 7, we recall basic concepts and results about coarse geometry on metric spaces, which will be needed in the study of leaves. Some new concepts and results are also given. To begin with, this chapter is devoted to the study of coarse quasi-isometries, and other related classes of maps. They define the primary relation we want to explore on a compact foliated space, being in coarsely quasiisometric leaves.

\subsection{Notation, conventions and terminology}

The contents of this section applies to Chapters 27.

Symbols $M, M^{\prime}$ and $M^{\prime \prime}$ will denote metric spaces with attendant metrics $d$, $d^{\prime}$ and $d^{\prime \prime}$, respectively; $\left\{M_{i}\right\}_{i \in I}$ and $\left\{M_{i}^{\prime}\right\}$ will denote classes of metric spaces with the same index $I=\{i\}$. Unless otherwise stated, a subset of a metric space becomes a metric space with the induced metric.

Let $r, s \geq 0, x \in M$ and $S, T \subset M$. The open and closed balls in $M$ of center $x$ and radius $r$, defined by $d_{M}(x, \cdot)<r$ and $d_{M}(x, \cdot) \leq r$, are denoted by $B_{M}(x, r)$ and $\bar{B}_{M}(x, r)$, respectively; in particular, $\bar{B}_{M}(x, 0)=\{x\}$, and $B_{M}(x, 0)=\varnothing$. The penumbrd ${ }^{1}$ of $S$ of radius $r$ is the set

$$
\operatorname{Pen}_{M}(S, r)=\bigcup_{y \in S} \bar{B}_{M}(y, r) .
$$

In particular, $\bar{B}_{M}(x, r)=\operatorname{Pen}_{M}(\{x\}, r)$. The terms open/closed $r$-ball and $r$ penumbra will be also used to indicate the radius $r$. Obviously,

$$
\operatorname{Pen}_{M}(S \cap T, r) \subset \operatorname{Pen}_{M}(S, r) \cap \operatorname{Pen}_{M}(T, r) .
$$

Moreover, by the triangle inequality,

$$
\operatorname{Pen}_{M}\left(\operatorname{Pen}_{M}(S, r), s\right) \subset \operatorname{Pen}_{M}(S, r+s)
$$

for all $r, s>0$, and

$$
r<s \Longrightarrow \operatorname{Pen}_{M}(\bar{S}, r) \subset \overline{\operatorname{Pen}_{M}(S, r)} \subset \operatorname{Pen}_{M}(S, s),
$$

where the first inclusion is an equality if $M$ is proper ${ }^{2}$. The $r$-boundary ${ }^{3}$ of $S$ is the set

$$
\partial_{r}^{M} S=\operatorname{Pen}_{M}(S, r) \cap \operatorname{Pen}_{M}(M \backslash S, r) ;
$$

in particular, $\partial_{0}^{M} S=\varnothing$. The notation $B(x, r), \bar{B}(x, r), \operatorname{Pen}(S, r)$ and $\partial_{r} S$ can be also used if it is clear which metric space is being considered. The inclusion

$$
\partial_{r} \operatorname{Pen}(S, s) \subset \partial_{r+s} S
$$

\footnotetext{
${ }^{1}$ This is slightly different from the definition of this concept given in $\mathbf{8 5}$.

${ }^{2}$ Recall that $M$ is called proper if its closed balls are compact

${ }^{3}$ This is also slightly different from the definition of this concept given in $\mathbf{2 0}$.
} 
can be proved as follows. For each $x \in \partial_{r} \operatorname{Pen}(S, s)$, there are $y \in \operatorname{Pen}(S, s)$ and $z \in M \backslash \operatorname{Pen}(S, s) \subset M \backslash S$ such that $d(x, y) \leq r$ and $d(x, z) \leq r$. Then there is $y_{0} \in S$ such that $d\left(y, y_{0}\right) \leq s$, obtaining $d\left(x, y_{0}\right) \leq r+s$ by the triangle inequality. Thus $x \in \partial_{r+s} S$.

\subsection{Coarse quasi-isometries}

A map $f: M \rightarrow M^{\prime}$ is Lipschitz if there is some $C>0$ such that $d^{\prime}\left(f(x), f\left(x^{\prime}\right)\right) \leq$ $C d(x, y)$ for all $x, y \in M$. Such a $C$ will be called a Lipschitz distortion of $f$. The map $f$ is called bi-Lipschitz when there is $C \geq 1$ such that

$$
\frac{1}{C} d(x, y) \leq d^{\prime}\left(f(x), f\left(x^{\prime}\right)\right) \leq C d(x, y)
$$

for all $x, y \in M$. In this case, $C$ will be called a bi-Lipschitz distortion of $f$. The term $C$-(bi)-Lipschitz may be also used for a (bi-)Lipschitz map with (bi-)Lipschitz distortion $C$. A 1-Lipschitz map is called non-expanding. A class of (bi-)Lipschitz maps is called equi-(bi-)Lipschitz when they have some common (bi-)Lipschitz distortion. Two metrics $d_{1}$ and $d_{2}$ on a set $S$ are called Lipschitz equivalent if the identity map $\left(S, d_{1}\right) \rightarrow\left(S, d_{2}\right)$ is bi-Lipschitz. Let $\left\{S_{i}\right\}$ be a class of sets each endowed with two metrics $d_{i, 1}$ and $d_{i, 2}$; the class $\left\{d_{i, 1}\right\}$ is equi-Lipschitz equivalent to $\left\{d_{i, 2}\right\}$ if, for all $i$, the identity maps $\left(S_{i}, d_{i, 1}\right) \rightarrow\left(S_{i}, d_{i, 2}\right)$ are equi-bi-Lipschitz.

REMARK 2.1. Any bi-Lipschitz map is injective. If $f: M \rightarrow M^{\prime}$ and $f^{\prime}: M^{\prime} \rightarrow$ $M^{\prime \prime}$ are (bi-)Lipschitz maps with respective (bi-)Lipschitz distortions $C$ and $C^{\prime}$, then $f^{\prime} f: M \rightarrow M^{\prime \prime}$ is a (bi-)Lipschitz map with (bi-)Lipschitz distortion $C C^{\prime}$.

A subset $A$ of $M$ is called a net in $M$ if there is $K \geq 0$ such that $\operatorname{Pen}_{M}(A, K)=$ $M$. A subset $A$ of $M$ is said to be separated if there is $\delta>0$ such that $d(x, y)>\delta$ for every $x \neq y$ in $A$. The terms $K$-ne $\rrbracket$ and $\delta$-separated will be also used. If $\left\{M_{i}\right\}$ is a class of metric spaces, a class of subsets $\left\{A_{i} \subset M_{i}\right\}$ is called an equi-net if there is $K \geq 0$ such that every $A_{i}$ is a $K$-net in $M_{i}$.

Remark 2.2. If $A$ is a $K$-net in $M$, then it is a $K$-net in any subset of $M$ that contains $A$. By the triangle inequality, if $A_{1}$ is a $K_{1}$-net in $M$, and $A_{2}$ is a $K_{2}$-net in $A_{1}$, then $A_{2}$ is a $\left(K_{1}+K_{2}\right)$-net in $M$. If $f: M \rightarrow M^{\prime}$ is a bi-Lipschitz bijection with bi-Lipschitz distortion $C$, and $A$ is a $K$-net in $M$, then $f(A)$ is a $C K$-net in $M^{\prime}$.

Lemma 2.3 (Álvarez-Candel [1, Lemma 2.1]). Let $K>0$ and $x_{0} \in M$. There is some $K$-separated $K$-net $A$ of $M$ so that $x_{0} \in A$.

Remark 2.4. In [11, Lemma 2.1], it is not explicitly stated that $x_{0} \in A$. In that proof, $A$ is a maximal element of the family of $K$-separated subsets of $M$. But the proof works as well using the family of $K$-separated subsets of $M$ that contain $x_{0}$, obtaining Lemma 2.3 In fact, it can be similarly proved that there is some $K$-separated $K$-net containing any given $K$-separated subset.

Definition 2.5 (Gromov [55). A coarse quasi-isometry of $M$ to $M^{\prime}$ is a biLipschitz bijection $f: A \rightarrow A^{\prime}$, where $A$ and $A^{\prime}$ are nets in $M$ and $M^{\prime}$, respectively; in this case, $M$ and $M^{\prime}$ are said to have the same coarse quasi-isometry type or to be coarsely quasi-isometric. If $A$ and $A^{\prime}$ are $K$-nets, and $C$ is a bi-Lipschitz

\footnotetext{
${ }^{4}$ The definition of $K$-net is slightly different from the definition used in [1]. Our arguments become simpler in this way.
} 
distortion of $f$, then the pair $(K, C)$ is called a coarse distortion of $f$; the term $(K, C)$-coarse quasi-isometry may be also used in this case. It is said that a map $M \rightarrow M^{\prime}$ induces a coarse quasi-isometry when its restriction to some subsets of $M$ and $M^{\prime}$ is a coarse quasi-isometry of $M$ to $M^{\prime}$. A coarse quasi-isometry of $M$ to itself will be called a coarsely quasi-isometric transformation of $M$.

REMARK 2.6. If $f: A \rightarrow A^{\prime}$ is a $(K, C)$-coarse quasi-isometry of $M$ to $M^{\prime}$, then $f^{-1}: A^{\prime} \rightarrow A$ is a $(K, C)$-coarse quasi-isometry of $M^{\prime}$ to $M$. If moreover $g: B^{\prime} \rightarrow B^{\prime \prime}$ is a $(K, C)$-coarse quasi-isometry of $M^{\prime}$ to $M^{\prime \prime}$, and $A^{\prime} \subset B^{\prime}$, then, using Remarks 2.1 and 2.2 , it is easy to check that $g f: A \rightarrow g\left(A^{\prime}\right)$ is a coarse quasi-isometry of $M$ to $M^{\prime \prime}$ with coarse distortion $\left(K+C K, C^{2}\right)$.

Definition 2.7. Let $f_{i}$ be a coarse quasi-isometry of each $M_{i}$ to $M_{i}^{\prime}$. If all of them have a common coarse distortion, then $\left\{f_{i}\right\}$ is called a family of equicoarse quasi-isometries. In this case, $\left\{M_{i}\right\}$ and $\left\{M_{i}^{\prime}\right\}$ are called equi-coarsely quasiisometric.

Definition 2.8. Two coarse quasi-isometries $f: A \rightarrow A^{\prime}$ and $g: B \rightarrow B^{\prime}$ of $M$ to $M^{\prime}$ are said to be close if there are $r, s \geq 0$ such that, for all $x \in A$, there is some $y \in B$ with $d(x, y) \leq r$ and $d^{\prime}(f(x), g(y)) \leq s$. (In this case, it may be also said that $f$ and $g$ are $(r, s)$-close, or that $f$ is $(r, s)$-close to $g$.)

Proposition 2.9. "Being close" is an equivalence relation on the set of coarse quasi-isometries of $M$ to $M^{\prime}$.

Proof. The relation "Being close" is obviously reflexive. To prove that it is symmetric, let $f: A \rightarrow A^{\prime}$ and $g: B \rightarrow B^{\prime}$ be coarse quasi-isometries of $M$ to $M^{\prime}$ such that $f$ is $(r, s)$-close to $g$. Let $(K, C)$ be a coarse distortion for $g$. For any $y \in B$, there is some $x \in A$ such that $d(x, y) \leq K$. Then there is some $y^{\prime} \in B$ such that $d\left(x, y^{\prime}\right) \leq r$ and $d^{\prime}\left(f(x), g\left(y^{\prime}\right)\right) \leq s$. It follows that

$$
\begin{aligned}
d^{\prime}(f(x), g(y)) \leq d^{\prime}\left(f(x), g\left(y^{\prime}\right)\right)+d^{\prime}( & \left.g\left(y^{\prime}\right), g(y)\right) \leq s+C d\left(y^{\prime}, y\right) \\
& \leq s+C\left(d\left(y^{\prime}, x\right)+d(x, y)\right) \leq s+C(r+K),
\end{aligned}
$$

obtaining that $g$ is $(K, s+C(r+K))$-close to $f$.

To prove that the relation "Being close" is transitive, let $f$ and $g$ be as above, and let $h: D \rightarrow D^{\prime}$ be a coarse quasi-isometry of $M$ to $M^{\prime}$ that is $(t, u)$-close to $g$. By the triangle inequality, it easily follows that $f$ is $(r+t, s+u)$-close to $h$.

\subsection{Coarse composites}

Let $f: A \rightarrow A_{1}^{\prime}$ and $f^{\prime}: A_{2}^{\prime} \rightarrow A^{\prime \prime}$ be coarse quasi-isometries of $M$ to $M^{\prime}$ and of $M^{\prime}$ to $M^{\prime \prime}$, respectively, and let $(K, C)$ be a coarse distortion for both. The following definition makes sense by Remark 2.6 .

Definition 2.10. A coarse composite of $f$ and $f^{\prime}$ is any coarse quasi-isometry of $M$ to $M^{\prime \prime}$ that is close to the composite $g^{\prime} g$, where $g$ (respectively, $g^{\prime}$ ) is a coarse quasi-isometry of $M$ to $M^{\prime}$ (respectively, of $M^{\prime}$ to $M^{\prime \prime}$ ) close to $f$ (respectively, $f^{\prime}$ ) such that im $g \subset \operatorname{dom} g^{\prime}$.

Proposition 2.11. Every two coarse composites of $f$ and $f^{\prime}$ are close.

Proof. Let $g$ and $h$ be coarse quasi-isometries of $M$ to $M^{\prime}$ close to $f$, and let $g^{\prime}$ and $h^{\prime}$ be coarse quasi-isometries of $M^{\prime}$ to $M^{\prime \prime}$ close to $f^{\prime}$. Suppose that 
$\operatorname{im} g \subset \operatorname{dom} g^{\prime}$ and $\operatorname{im} h \subset \operatorname{dom} h^{\prime}$. Then the composites $g^{\prime} g: \operatorname{dom} g \rightarrow g^{\prime}(\operatorname{im} g)$ and $h^{\prime} h: \operatorname{dom} h \rightarrow h^{\prime}(\operatorname{im} h)$ are coarse quasi-isometries of $M$ to $M^{\prime \prime}$ (Remark 2.6). By Proposition 2.9 there are $r, s, t, u \geq 0$ such that $g$ is $(r, s)$-close to $h$, and $g^{\prime}$ is $(t, u)$ close to $h^{\prime}$. Thus, for each $x \in \operatorname{dom} g$, there is some $y \in \operatorname{dom} h$ so that $d(x, y) \leq r$ and $d(g(x), h(y)) \leq s$. Then there is some $z^{\prime} \in \operatorname{dom} h^{\prime}$ such that $d^{\prime}\left(g(x), z^{\prime}\right) \leq t$ and $d^{\prime \prime}\left(g^{\prime} g(x), h^{\prime}\left(z^{\prime}\right)\right) \leq u$. Let $C$ be a bi-Lipschitz distortion of $h^{\prime}$. We get

$$
\begin{aligned}
& d^{\prime \prime}\left(g^{\prime} g(x), h^{\prime} h(y)\right) \leq d^{\prime \prime}\left(g^{\prime} g(x), h^{\prime}\left(z^{\prime}\right)\right)+d^{\prime \prime}\left(h^{\prime}\left(z^{\prime}\right), h^{\prime} h(y)\right) \\
& \quad \leq u+C d^{\prime}\left(z^{\prime}, h(y)\right) \leq u+C\left(d^{\prime}\left(z^{\prime}, g(x)\right)+d^{\prime}(g(x), h(y))\right) \leq u+C(t+s) .
\end{aligned}
$$

This shows that $g^{\prime} g$ is $(r, u+C(t+s))$-close to $h^{\prime} h$.

The existence of coarse composites is guaranteed by the following result.

Proposition 2.12. There is a coarse composite $g: B \rightarrow B^{\prime \prime}$ of $f$ and $f^{\prime}$ with coarse distortion $\left(K(5 C+1), 5 C^{2}\right)$ such that $B$ is a $5 K C$-net of $A, B^{\prime \prime}$ is a $3 K C$ net of $A^{\prime \prime}$, and $d^{\prime}\left(f(x), f^{\prime-1} g(x)\right) \leq 2 K$ for all $x \in B$. Furthermore, if $x_{1} \in A$ and $x_{2}^{\prime} \in A_{2}^{\prime}$ are given so that $d^{\prime}\left(f\left(x_{1}\right), x_{2}^{\prime}\right) \leq 2 K$, then $g$ can be chosen such that $x_{1} \in B$, $f^{\prime}\left(x_{2}^{\prime}\right) \in B^{\prime \prime}$ and $g\left(x_{1}\right)=f^{\prime}\left(x_{2}^{\prime}\right)$.

The following lemma will be used to prove Proposition 2.12

Lemma 2.13. For $K>0$, let $A_{1}$ and $A_{2}$ be $K$-nets of $M$. Then there is a $(6 K, 5)$-coarsely quasi-isometric transformation $h: B_{1} \rightarrow B_{2}$ of $M$ such that $B_{1}$ is a $5 K$-net of $A_{1}, B_{2}$ is a $3 K$-net of $A_{2}$, and $d(x, h(x)) \leq 2 K$ for all $x \in B_{1}$. Moreover, if $x_{1} \in A_{1}$ and $x_{2} \in A_{2}$ are given so that $d\left(x_{1}, x_{2}\right) \leq 2 K$, then $h$ can be chosen so that $x_{1} \in B_{1}, x_{2} \in B_{2}$ and $h\left(x_{1}\right)=x_{2}$.

Proof. By Lemma 2.3. $A_{k}$ has some $K$-separated $K$-net $A_{k}^{\prime}$ for $k \in\{1,2\}$. Then $A_{k}^{\prime}$ is a $2 K$-net of $M$ because $A_{k}$ is $K$-net of $M$ (Remark 2.2). So, for each $x \in A_{1}^{\prime}$, there is some point $h(x) \in A_{2}^{\prime}$ such that $d(x, h(x)) \leq 2 K$. A map $h: A_{1}^{\prime} \rightarrow A_{2}^{\prime}$ is defined in this way, and let $B_{2}$ denote its image. Choose some point $g(y) \in h^{-1}(y)$ for each $y \in B_{2}$, defining a map $g: B_{2} \rightarrow A_{1}^{\prime}$, whose image is denoted by $B_{1}$. Then the restriction $h: B_{1} \rightarrow B_{2}$ is bijective with inverse equal to the restriction $g: B_{2} \rightarrow B_{1}$.

According to Lemma 2.3 , given points $x_{k} \in A_{k}$ with $d\left(x_{1}, x_{2}\right) \leq 2 K$, we can take $A_{k}^{\prime}$ so that $x_{k} \in A_{k}^{\prime}$, and we can choose $h\left(x_{1}\right)=x_{2}$ and $g\left(x_{2}\right)=x_{1}$, obtaining that $x_{k} \in B_{k}$.

For each $z \in A_{1}$, there is $x \in A_{1}^{\prime}$ with $d(z, x) \leq K$. Then $h(x) \in B_{2}, g h(x) \in B_{1}$, and

$$
\begin{gathered}
d(z, h(x)) \leq d(z, x)+d(x, h(x)) \leq 3 K, \\
d(z, g h(x)) \leq d(z, h(x))+d(h(x), g h(x)) \leq 5 K .
\end{gathered}
$$

Thus $B_{1}$ is a $5 K$-net of $A_{1}$, and $B_{2}$ is a $3 K$-net of $A_{2}$. It follows that $B_{1}$ and $B_{2}$ are $6 K$-nets of $M$ because $A_{1}$ and $A_{2}$ are $K$-nets of $M$ (Remark 2.2). 
Since $B_{1}$ and $B_{2}$ are $K$-separated and $h: B_{1} \rightarrow B_{2}$ is bijective, for $x \neq y$ in $B_{1}$, we have

$$
\begin{aligned}
d(h(x), h(y)) \leq d(h(x), x)+d(x, y)+ & d(y, h(y)) \\
\leq d(x, y)+ & 4 K<5 d(x, y), \\
d(x, y) \leq d(x, h(x))+ & d(h(x), h(y))+d(h(y), y) \\
\leq & d(h(x), h(y))+4 K<5 d(h(x), h(y)) .
\end{aligned}
$$

of Proposition 2.12, By Lemma 2.13 there is a $(6 K, 5)$-coarsely quasi-isometric transformation $h: B_{1}^{\prime} \rightarrow B_{2}^{\prime}$ of $M^{\prime}$ such that $B_{1}^{\prime}$ is a $5 K$-net of $A_{1}^{\prime}, B_{2}^{\prime}$ is a $3 K$-net of $A_{2}^{\prime}$, and $d^{\prime}\left(x^{\prime}, h\left(x^{\prime}\right)\right) \leq 2 K$ for all $x^{\prime} \in B_{1}^{\prime}$. By Remark 2.2. $B=f^{-1}\left(B_{1}^{\prime}\right)$ is a $5 K C$-net of $A$, and $B^{\prime \prime}=f^{\prime}\left(B_{2}^{\prime}\right)$ is a $3 K C$-net of $A^{\prime \prime}$. Thus, by Remark $2.2, B$ and $B^{\prime}$ are $K(5 C+1)$-nets in $M$ and $M^{\prime}$ because $A$ and $A^{\prime \prime}$ are $K$-nets in $M$ and $M^{\prime \prime}$, respectively. Moreover the composite

$$
B \stackrel{f}{\longrightarrow} B_{1}^{\prime} \stackrel{h}{\longrightarrow} B_{2}^{\prime} \stackrel{f^{\prime}}{\longrightarrow} B^{\prime \prime}
$$

is a $5 C^{2}$-bi-Lipschitz bijection (Remark 2.1), denoted by $g: B \rightarrow B^{\prime \prime}$, which satisfies

$$
d^{\prime}\left(f(x), f^{\prime-1} g(x)\right)=d^{\prime}(f(x), h f(x)) \leq 2 K
$$

for each $x \in B$.

Observe that the coarse quasi-isometry $f: B \rightarrow B_{1}^{\prime}$ is $(0,0)$-close to $f: A \rightarrow A_{1}^{\prime}$ because $B \subset A$.

It will be now shown that the coarse quasi-isometry $f^{\prime} h: B_{1}^{\prime} \rightarrow B^{\prime \prime}$ is close to $f^{\prime}: A_{2}^{\prime} \rightarrow A^{\prime \prime}$. For each $x^{\prime} \in B_{1}^{\prime}$ there is $y^{\prime} \in A_{2}^{\prime}$ such that $d^{\prime}\left(x^{\prime}, y^{\prime}\right) \leq K$ because $A_{2}^{\prime}$ is a $K$-net in $M^{\prime}$. Furthermore

$$
d^{\prime \prime}\left(f^{\prime} h\left(x^{\prime}\right), f^{\prime}\left(y^{\prime}\right)\right) \leq C d^{\prime}\left(h\left(x^{\prime}\right), y^{\prime}\right) \leq C\left(d^{\prime}\left(h\left(x^{\prime}\right), x^{\prime}\right)+d^{\prime}\left(x^{\prime}, y^{\prime}\right)\right) \leq 3 K C,
$$

obtaining that $f^{\prime} h: B_{1}^{\prime} \rightarrow B^{\prime \prime}$ is $(K, 3 K C)$-close to $f^{\prime}: A_{2}^{\prime} \rightarrow A^{\prime \prime}$.

Fix $x_{1} \in A$ and $x_{2}^{\prime} \in A_{2}^{\prime}$ so that $d^{\prime}\left(f\left(x_{1}\right), x_{2}^{\prime}\right) \leq 2 K$. By Lemma 2.13, we can choose $h$ such that $f\left(x_{1}\right) \in B_{1}^{\prime}, x_{2}^{\prime} \in B_{2}^{\prime}$ and $h f\left(x_{1}\right)=x_{2}^{\prime}$. Hence $x_{1} \in B, f^{\prime}\left(x_{2}^{\prime}\right) \in B^{\prime \prime}$ and $g\left(x_{1}\right)=f^{\prime}\left(x_{2}^{\prime}\right)$.

According to Propositions 2.9, 2.12 and 2.11, the closeness classes of coarse quasi-isometries between metric spaces form a category of isomorphisms with the operation induced by coarse composite. The following direct consequence is well known.

COROllary 2.14. "Being coarsely quasi-isometric" is an equivalence relation on metric spaces.

\subsection{A coarsely quasi-isometric version of Arzela-Ascoli theorem}

The following proposition will be useful to produce coarse quasi-isometries. It is a version of the Arzela-Ascoli theorem for coarse quasi-isometries.

Proposition 2.15. Let $\left\{F_{n} \subset M\right\}$ and $\left\{F^{\prime}{ }_{n} \subset M^{\prime}\right\}$ be increasing sequences of finite subsets such that $\cup_{n} F_{n}$ and $\cup_{n} F^{\prime}{ }_{n}$ are L-nets in $M$ and $M^{\prime}$, respectively. For each $n$, let $f_{n}$ be a $(K, C)$-coarse quasi-isometry of $F_{n}$ to $F_{n}^{\prime}$, such that $f_{n}\left(F_{m} \cap\right.$ $\left.\operatorname{dom} f_{n}\right)=F_{m}^{\prime} \cap \operatorname{im} f_{n}$ if $m<n$. Then there is a $(K+L, C)$-coarse quasi-isometry $g$ of 
$M$ to $M^{\prime}$, which is the combination of restrictions $f_{n_{m}}: F_{m} \cap \operatorname{dom} f_{n_{m}} \rightarrow F_{m}^{\prime} \cap \operatorname{im} f_{n_{m}}$ for some subsequence $f_{n_{m}}$ with $n_{m} \geq m$.

Proof. For each $m$, let $\mathcal{C}_{m}$ be the set of restrictions $f_{n}: F_{m} \cap \operatorname{dom} f_{n} \rightarrow$ $F_{m}^{\prime} \cap \operatorname{im} f_{n}$ for all $n \geq m$. Define a graph structure on $\mathcal{C}=\sqcup_{m} \mathcal{C}_{m}$ by placing an edge between each $f \in \mathcal{C}_{m+1}$ and its restriction $F_{m} \cap \operatorname{dom} f \rightarrow F_{m}^{\prime} \cap \operatorname{im} f$, which is well defined and belongs to $\mathcal{C}_{m}$; such a $\mathcal{C}$ is an infinite tree. Each $\mathcal{C}_{m}$ is finite because so are the sets $F_{m}$ and $F_{m}^{\prime}$, and thus each vertex of $\mathcal{C}$ meets a finite number of edges. Therefore $\mathcal{C}$ contains an infinite ray with vertices $g_{m} \in \mathcal{C}_{m}$; every $g_{m}$ is a restriction $f_{n_{m}}: F_{m} \cap \operatorname{dom} f_{n_{m}} \rightarrow F_{m}^{\prime} \cap \operatorname{im} f_{n_{m}}$ with $n_{m} \geq n$. All maps $g_{m}$ can be combined to define a map $g: \bigcup_{m} \operatorname{dom} g_{m} \rightarrow \cup_{m} \mathrm{im} g_{m}$, which is a $(K, C)$-coarse quasi-isometry of $\cup_{n} F_{n}$ to $\cup_{n} F^{\prime}{ }_{n}$. Since $\cup_{n} F_{n}$ and $\cup_{n} F^{\prime}{ }_{n}$ are $L$-nets in $M$ and $M^{\prime}$, respectively, this $g$ is a $(K+L, C)$-coarse quasi-isometry of $M$ to $M^{\prime}$ (Remark 2.2.

REMARK 2.16. In Proposition 2.15, observe that, if $x \in \bigcap_{n} \operatorname{dom} f_{n}$ and $f_{n}(x)=y$ for all $n$, then $x \in \operatorname{dom} g$ and $g(x)=y$.

\subsection{Large scale Lipschitz maps}

Definition 2.17. Two maps, $f, g: S \rightarrow M$, of a set, $S$, into a metric space, $M$, are said to be $\operatorname{clos} \epsilon^{5}$ if there is some $c \geq 0$ such that $d(f(x), g(x)) \leq c$ for all $x \in S$; it may be also said that $f$ and $g$ are $c$-close, or that $f$ is $c$-close to $g$.

REMARK 2.18. "Being close" is an equivalence relation on the set of maps of a set to a metric space.

Definition 2.19 (Gromov [55]). A man ${ }^{6} \phi: M \rightarrow M^{\prime}$ is said to be large scale Lipschitz if there exist $\lambda>0$ and $b \geq 0$ such that

$$
d^{\prime}(\phi(x), \phi(y)) \leq \lambda d(x, y)+b
$$

for all $x, y \in M$; in this case, the pair $(\lambda, b)$ is called a large scale Lipschitz distortion of $\phi$, and $\phi$ is said to be $(\lambda, b)$-large scale Lipschitz. The map $\phi$ is said to be large scale bi-Lipschitz if there exist constants $\lambda>0$ and $b \geq 0$ such that

$$
\frac{1}{\lambda}(d(x, y)-b) \leq d^{\prime}(\phi(x), \phi(y)) \leq \lambda d(x, y)+b
$$

for all $x, y \in M$; in this case, the pair $(\lambda, b)$ is called a large scale bi-Lipschitz distortion of $\phi$, and $\phi$ is said to be $(\lambda, b)$-large scale bi-Lipschitz.

A map $\phi: M \rightarrow M^{\prime}$ is said to be a large scale Lipschitz equivalence if it is large scale Lipschitz and there is another large scale Lipschitz map $\psi: M^{\prime} \rightarrow M$ so that $\psi \phi$ and $\phi \psi$ are close to $\operatorname{id}_{M}$ and $\operatorname{id}_{M^{\prime}}$, respectively. In this case, if $(\lambda, b)$ is a large scale Lipschitz distortion of $\phi$ and $\psi$, and $\psi \phi$ and $\phi \psi$ are $c$-close to the identity maps, then $(\lambda, b, c)$ is called a large scale Lipschitz equivalence distortion of $\phi$; it may be also said that $\phi$ is a $(\lambda, b, c)$-large scale Lipschitz equivalence. In this case, $M$ and $M^{\prime}$ are said to be large scale Lipschitz equivalent. Two metrics $d_{1}$ and $d_{2}$ on a set $S$ are called large scale Lipschitz equivalent if the identity map $\left(S, d_{1}\right) \rightarrow\left(S, d_{2}\right)$ is large scale bi-Lipschitz.

\footnotetext{
${ }^{5}$ This terminology is used in $\mathbf{6 5}$. Other terms used to indicate the same property are coarsely equivalent 85, parallel [55, bornotopic 84, and uniformly close [20.

${ }^{6}$ Continuity is not assumed here.
} 
Definition 2.20. Let $\phi_{i}: M_{i} \rightarrow M_{i}^{\prime}$ for each $i$. The class $\left\{\phi_{i}\right\}$ is said to be equi-large scale (bi-)Lipschitz if all the maps $\phi_{i}$ are large scale (bi-)Lipschitz with a common large scale (bi-)Lipschitz distortion. The class $\left\{\phi_{i}\right\}$ is said to be equi-large scale Lipschitz equivalences if the maps $\phi_{i}$ are large scale Lipschitz equivalences and have a common large scale Lipschitz equivalence distortion; in this case, $\left\{M_{i}\right\}$ and $\left\{M_{i}^{\prime}\right\}$ are called equi-large scale Lipschitz equivalent. Given a class of sets $\left\{S_{i}\right\}$, and metrics $d_{i, 1}$ and $d_{i, 2}$ on each $S_{i},\left\{d_{i, 1}\right\}$ is said to be equi-large scale Lipschitz equivalent to $\left\{d_{i, 2}\right\}$ if the identity maps $\left(S_{i}, d_{i, 1}\right) \rightarrow\left(S_{i}, d_{i, 2}\right)$ are equi-large scale bi-Lipschitz.

The qualitative content of the following lemmas is well known, but we keep track of the constants involved.

Lemma 2.21. If $\phi: M \rightarrow M^{\prime}$ is a $(\lambda, b, c)$-large scale Lipschitz equivalence, then $\phi$ is $(\lambda, b+2 c)$-large scale bi-Lipschitz.

Proof. Let $\psi: M^{\prime} \rightarrow M$ be a $(\lambda, b)$-large scale Lipschitz map such that $\psi \phi$ and $\phi \psi$ are $c$-close to $\operatorname{id}_{M}$ and $\operatorname{id}_{M^{\prime}}$, respectively. Then, for all $x, y \in M$,

$$
d(x, y) \leq d(\psi \phi(x), \psi \phi(y))+2 c \leq \lambda d^{\prime}(\phi(x), \phi(y))+b+2 c,
$$

by the triangle inequality.

Lemma 2.22. Let $\phi: M \rightarrow M^{\prime}$ be $(\lambda, b)$-large scale bi-Lipschitz. If $\phi(M)$ is a $c$-net in $M^{\prime}$, then $\phi$ is a $(\lambda, b+2 \lambda c, \max \{b, c\})$-large scale Lipschitz equivalence.

Proof. Because $\phi(M)$ is a $c$-net in $M^{\prime}$, a map $\psi: M^{\prime} \rightarrow M$ can be constructed by choosing, for each $x^{\prime} \in M^{\prime}$, one point $\psi\left(x^{\prime}\right) \in M$ such that $d^{\prime}\left(x^{\prime}, \phi \psi\left(x^{\prime}\right)\right) \leq c$, and furthermore so that, if $x^{\prime} \in \phi(M)$, then $\phi \psi\left(x^{\prime}\right)=x^{\prime}$; i.e., $\phi \psi \phi=\phi$.

Then, for all $x^{\prime}, y^{\prime} \in M^{\prime}$ and $x \in M$,

$$
\begin{aligned}
d(x, \psi \phi(x)) & \leq \lambda d^{\prime}(\phi(x), \phi \psi \phi(x))+b=\lambda d^{\prime}(\phi(x), \phi(x))+b=b, \\
d^{\prime}\left(x^{\prime}, y^{\prime}\right) & \leq d^{\prime}\left(x^{\prime}, \phi \psi\left(x^{\prime}\right)\right)+d^{\prime}\left(\phi \psi\left(x^{\prime}\right), \phi \psi\left(y^{\prime}\right)\right)+d^{\prime}\left(\phi \psi\left(y^{\prime}\right), y^{\prime}\right) \\
& \leq \lambda d\left(\psi\left(x^{\prime}\right), \psi\left(y^{\prime}\right)\right)+b+2 c, \\
d\left(\psi\left(x^{\prime}\right), \psi\left(y^{\prime}\right)\right) & \leq \lambda d^{\prime}\left(\phi \psi\left(x^{\prime}\right), \phi \psi\left(y^{\prime}\right)\right)+b \\
& \leq \lambda\left(d^{\prime}\left(\phi \psi\left(x^{\prime}\right), x^{\prime}\right)+d^{\prime}\left(x^{\prime}, y^{\prime}\right)+d^{\prime}\left(y^{\prime}, \phi \psi\left(y^{\prime}\right)\right)\right)+b \\
& \leq \lambda d^{\prime}\left(x^{\prime}, y^{\prime}\right)+b+2 \lambda c .
\end{aligned}
$$

REMARK 2.23. According to Lemmas 2.21 and 2.22, (equi-) large scale Lipschitz equivalences are just (equi-) large scale bi-Lipschitz maps whose images are (equi-) nets.

Lemma 2.24. Let $\phi: M \rightarrow M^{\prime}$ and $\phi^{\prime}: M^{\prime} \rightarrow M^{\prime \prime}$ be maps. The following properties hold:

(i) If $\phi$ and $\phi^{\prime}$ are $(\lambda, b)$-large scale Lipschitz, then $\phi^{\prime} \phi$ is $\left(\lambda^{2}, \lambda b+b\right)$-large scale Lipschitz.

(ii) If $\phi$ and $\phi^{\prime}$ are $(\lambda, b, c)$-large scale Lipschitz equivalences, then $\phi^{\prime} \phi$ is a $\left(\lambda^{2}, \lambda b+\right.$ $b, 2 c)$-large scale Lipschitz equivalence. 
Proof. Property (i) is true because, for all $x, y \in M$,

$$
d^{\prime \prime}\left(\phi^{\prime} \phi(x), \phi^{\prime} \phi(y)\right) \leq \lambda d^{\prime}(\phi(x), \phi(y))+b \leq \lambda^{2} d(x, y)+\lambda b+b .
$$

To prove (ii), take $(\lambda, b)$-large scale Lipschitz maps $\psi: M^{\prime} \rightarrow M$ and $\psi^{\prime}: M^{\prime \prime} \rightarrow$ $M^{\prime}$ such that $\psi \phi$ is $c$-close to $\operatorname{id}_{M}, \phi \psi$ and $\psi^{\prime} \phi^{\prime}$ are $c$-close to $\operatorname{id}_{M^{\prime}}$, and $\phi^{\prime} \psi^{\prime}$ is $c$-close to $\operatorname{id}_{M^{\prime \prime}}$. By (i), $\phi^{\prime} \phi$ and $\psi \psi^{\prime}$ are $\left(\lambda^{2}, \lambda b+b\right)$-large scale Lipschitz. Moreover

$$
d\left(\psi \psi^{\prime} \phi^{\prime} \phi(x), x\right) \leq d\left(\psi \psi^{\prime} \phi^{\prime} \phi(x), \phi^{\prime} \phi(x)\right)+d\left(\phi^{\prime} \phi(x), x\right) \leq 2 c
$$

for all $x \in M$, obtaining that $\psi \psi^{\prime} \phi^{\prime} \phi$ is $2 c$-close to $\mathrm{id}_{M}$. Similarly, $\phi^{\prime} \phi \psi \psi^{\prime}$ is $2 c$-close to $\operatorname{id}_{M^{\prime \prime}}$.

Lemma 2.25. Let $\phi, \psi: M \rightarrow M^{\prime}$ and $\phi^{\prime}, \psi^{\prime}: M^{\prime} \rightarrow M^{\prime \prime}$ be $(\lambda, b)$-large scale Lipschitz maps. If $\phi$ and $\phi^{\prime}$ are $R$-close to $\psi$ and $\psi^{\prime}$, respectively, then $\phi^{\prime} \phi$ is $(\lambda R+b+R)$-close to $\psi^{\prime} \psi$.

Proof. For all $x \in M$,

$$
\begin{aligned}
d^{\prime \prime}\left(\phi^{\prime} \phi(x), \psi^{\prime} \psi(x)\right) \leq d^{\prime \prime}\left(\phi^{\prime} \phi(x), \psi^{\prime} \phi(x)\right)+d^{\prime \prime}\left(\psi^{\prime} \phi(x), \psi^{\prime} \psi(x)\right) & \\
& \leq R+\lambda d^{\prime}(\phi(x), \psi(x))+b \leq \lambda R+b+R .
\end{aligned}
$$

By Lemmas 2.24 and 2.25, the closeness classes of large scale Lipschitz maps between metric spaces form a category, whose isomorphisms are the classes represented by large scale Lipschitz equivalences.

It is well known that two metric spaces are coarsely quasi-isometric if and only if they are isomorphic in the category whose objects are metric spaces and whose morphisms are closeness equivalence classes of large scale Lipschitz maps. This is part of the content of the following two results, where the constants involved are specially analyzed.

Proposition 2.26 (Álvarez-Candel [11, Proposition 2.2]). Any $(K, C)$-coarse quasi-isometry $f: A \rightarrow A^{\prime}$ of $M$ to $M^{\prime}$ is induced by a $(C, 2 C K, K)$-large scale Lipschitz equivalence $\phi: M \rightarrow M^{\prime}$.

Proposition 2.27 (Álvarez-Candel [11, Proposition 2.3]). For each $\varepsilon>0$ and $x_{0} \in M$, every $(\lambda, b, c)$-large scale Lipschitz equivalence $\phi: M \rightarrow M^{\prime}$ induces a $(K, C)$-coarse quasi-isometry $f: A \rightarrow A^{\prime}$ of $M$ to $M^{\prime}$ such that $x_{0} \in A$, where

$$
K=c+2 \lambda c+\lambda b+\lambda \varepsilon+b, \quad C=\lambda+\frac{\lambda}{\varepsilon}(2 c+b) .
$$

REMARK 2.28. In [11, Proposition 2.3], it is not explicitly stated that $A$ contains any given point $x_{0}$. But, in the proof of that proposition, the set $A$ is any $(2 c+b+\varepsilon)$-separated $(2 c+b+\varepsilon)$-net of $M$, and so, using Lemma 2.3 , it may be further impossed that $x_{0} \in A$.

REMARK 2.29. According to Propositions 2.26 and 2.27, $\left\{M_{i}\right\}$ and $\left\{M_{i}^{\prime}\right\}$ are equi-coarsely quasi-isometric if and only if they are equi-large scale Lipschitz equivalent.

Proposition 2.30. Let $\phi, \psi: M \rightarrow M^{\prime}$ be $(\lambda, b)$-large scale Lipschitz equivalences, and let $f: A \rightarrow A^{\prime}$ and $g: B \rightarrow B^{\prime}$ be $(K, C)$-coarse quasi-isometries of $M$ to $M^{\prime}$ induced by $\phi$ and $\psi$, respectively. The following properties hold:

(i) If $\phi$ is $R$-close to $\psi$, then $f$ is $(K, R+\lambda K+b)$-close to $g$. 
(ii) If $f$ is $(r, s)$-close to $g$, then $\phi$ is $(\lambda(r+2 K)+s+2 b)$-close to $\psi$.

Proof. (i) For all $x \in A$, there is some $y \in B$ so that $d(x, y) \leq K$. Then

$$
\begin{aligned}
d^{\prime}(f(x), g(y))=d^{\prime}(\phi(x), \psi(y)) \leq d^{\prime}(\phi(x), \psi(x)) & +d^{\prime}(\psi(x), \psi(y)) \\
\leq & R+\lambda d(x, y)+b \leq R+\lambda K+b .
\end{aligned}
$$

(ii) For any $x \in M$, there is some $y \in A$ such that $d(x, y) \leq K$. Then there is some $z \in B$ so that $d(y, z) \leq r$ and $d^{\prime}(f(y), g(z)) \leq s$. Hence

$$
\begin{aligned}
d^{\prime}(\phi(x), \psi(x)) \leq d^{\prime}(\phi(x), \phi(y))+d^{\prime}(f(y), g(z))+d^{\prime}(\psi(z), \psi(x)) & \\
\leq \lambda d(x, y)+s+\lambda d(z, x)+2 b \leq \lambda K+s+\lambda(d(z, y) & +d(y, x))+2 b \\
& \leq \lambda(r+2 K)+s+2 b .
\end{aligned}
$$

By Propositions 2.26, 2.27 and 2.30, the category whose objects are metric space and whose morphisms are closeness equivalence classes of coarse quasi-isometries between metric spaces can be identified to the subcategory of isomorphisms of the category of closeness classes of large scale Lipschitz maps between metric spaces. Therefore coarse quasi-isometries and large scale Lipschitz equivalences are equivalent concepts. We will often use large scale Lipschitz equivalences in the proofs because they become simpler. However direct proofs for coarse quasi-isometries may produce better constants. This will be indicated in remarks.

Proposition 2.31. Let $\phi: M \rightarrow M^{\prime}, x_{0} \in M, x_{0}^{\prime} \in M^{\prime}$ and $R \geq d^{\prime}\left(\phi\left(x_{0}\right), x_{0}^{\prime}\right)$, and let $\bar{\phi}: M \rightarrow M^{\prime}$ be defined by $\bar{\phi}\left(x_{0}\right)=x_{0}^{\prime}$ and $\bar{\phi}(x)=\phi(x)$ if $x \neq x_{0}$. The following properties hold:

(i) If $\phi$ is $(\lambda, b)$-large scale Lipschitz, then $\bar{\phi}$ is $(\lambda, b+R)$-large scale Lipschitz.

(ii) If $\phi$ is $a(\lambda, b, c)$-large scale Lipschitz equivalence, then $\bar{\phi}$ is $a(\lambda, \bar{b}, \bar{c})$-large scale Lipschitz equivalence, where $\bar{b}=b+R$ and $\bar{c}=\lambda R+b+2 c$.

Proof. Since $\bar{\phi}$ equals $\phi$ on $M \backslash\left\{x_{0}\right\}$, it is enough to check (i) for $x_{0}$ and any $x \neq x_{0}$ in $M$ :

$$
\begin{aligned}
d^{\prime}\left(\bar{\phi}\left(x_{0}\right), \bar{\phi}(x)\right)=d^{\prime}\left(x_{0}^{\prime}, \phi(x)\right) \leq d^{\prime}\left(x_{0}^{\prime}, \phi\left(x_{0}\right)\right)+d^{\prime}\left(\phi\left(x_{0}\right), \phi(x)\right) & \\
& \leq R+\lambda d\left(x_{0}, x\right)+b .
\end{aligned}
$$

To prove (ii), take a $(\lambda, b)$-large scale Lipschitz map $\psi: M^{\prime} \rightarrow M$ so that $\psi \phi$ and $\phi \psi$ are $c$-close to $\operatorname{id}_{M}$ and $\operatorname{id}_{M^{\prime}}$, respectively. Let $\bar{\psi}: M^{\prime} \rightarrow M$ be defined by $\bar{\psi}\left(x_{0}^{\prime}\right)=x_{0}$ and $\bar{\psi}\left(x^{\prime}\right)=\psi\left(x^{\prime}\right)$ if $x^{\prime} \neq x_{0}^{\prime}$. Then $\bar{\phi}$ and $\bar{\psi}$ are $(\lambda, b+R)$-large scale Lipschitz by (i). Moreover $\phi$ and $\psi$ are $(\lambda, b+2 c)$-large scale bi-Lipschitz by Lemma 2.21. Since $\phi$ and $\bar{\psi}$ equal $\phi$ and $\psi$ on $M \backslash\left\{x_{0}\right\}$ and $M^{\prime} \backslash\left\{x_{0}^{\prime}\right\}$, respectively, to check that $\bar{\psi} \bar{\phi}$ is $(\lambda R+b+2 c)$-close to $\operatorname{id}_{M}$, the only non-trivial case is at every point $x \in M$ with $\phi(x)=x_{0}^{\prime}$ :

$$
d(x, \bar{\psi} \bar{\phi}(x))=d\left(x, x_{0}\right) \leq \lambda d^{\prime}\left(\phi(x), \phi\left(x_{0}\right)\right)+b+2 c \leq \lambda R+b+2 c .
$$

Similarly, we get that $\bar{\phi} \bar{\psi}$ is $(\lambda R+b+2 c)$-close to $\operatorname{id}_{M^{\prime}}$.

Remark 2.32. In Proposition 2.31, note that $\bar{\phi}$ is $R$-close to $\phi$. 
Corollary 2.33. Let $f: A \rightarrow A^{\prime}$ be a $(K, C)$-coarse quasi-isometry of $M$ to $M^{\prime}$. Let $R>0, x_{0} \in M$ and $x_{0}^{\prime} \in M^{\prime}$ such that there is some $y_{0} \in A$ with $d\left(x_{0}, y_{0}\right) \leq R$ and $d^{\prime}\left(x_{0}^{\prime}, f\left(y_{0}\right)\right) \leq R$. Then, for all $\varepsilon>0$, there is a $(\bar{K}, \bar{C})$-coarse quasi-isometry $\bar{f}: B \rightarrow B^{\prime}$ of $M$ to $M^{\prime}(r, s)$-close to $f$ such that $x_{0} \in B, x_{0}^{\prime} \in B^{\prime}$ and $f\left(x_{0}\right)=x_{0}^{\prime}$, where

$$
\begin{gathered}
\bar{K}=\bar{c}+2 C \bar{c}+C \bar{b}+C \varepsilon+\bar{b}, \quad \bar{C}=C+\frac{C}{\varepsilon}(2 \bar{c}+\bar{b}), \\
r=\bar{K}, \quad s=C R+2 C K+R+\bar{C} \bar{K}+\bar{b} .
\end{gathered}
$$

Proof. By Proposition 2.26, $f$ is induced by a $(C, 2 C K, K)$-large scale Lipschitz equivalence $\phi: M \rightarrow M^{\prime}$. We have

$$
\begin{aligned}
d^{\prime}\left(\phi\left(x_{0}\right), x_{0}^{\prime}\right) \leq d^{\prime}\left(\phi\left(x_{0}\right), \phi\left(y_{0}\right)\right)+ & d^{\prime}\left(\phi\left(y_{0}\right), x_{0}^{\prime}\right) \\
& \leq C d\left(x_{0}, y_{0}\right)+2 C K+R \leq C R+2 C K+R .
\end{aligned}
$$

According to Proposition 2.31 and Remark 2.32, $\phi$ is $(C R+2 C K+R)$-close to a $(C, \bar{b}, \bar{c})$-large scale Lipschitz equivalence $\phi: M \rightarrow M^{\prime}$ with $\bar{\phi}\left(x_{0}\right)=x_{0}^{\prime}$. By Propositions 2.27 and 2.30 (1), for each $\varepsilon>0, \bar{\phi}$ induces a $(\bar{K}, \bar{C})$-coarse quasiisometry $\bar{f}: \bar{B} \rightarrow B^{\prime}$ of $\bar{M}$ to $M^{\prime}(\bar{K}, s)$-close to $f$ such that $x_{0} \in B$.

REMARK 2.34. Another version of Corollary 2.33, where

$$
\bar{K}=2 C^{2} R+2 C R+R+K, \quad \bar{C}=2 C+1, \quad r=s=R,
$$

can be proved without passing to large scale Lipschitz equivalences, with more involved arguments. These constants are simpler, but the constants of Corollary 2.33 give the following extra information: we can get $\bar{C}$ as close to $C$ as desired at the expense of increasing $\bar{K}$ (by taking $\varepsilon$ large enough).

\subsection{Coarse and rough maps}

Definition 2.35. A map $f: M \rightarrow M^{\prime}$ is called:

- uniformly expansive 7 if, for each $r \geq 0$, there is some $s_{r} \geq 0$ such that

$$
d(x, y) \leq r \Longrightarrow d^{\prime}(f(x), f(y)) \leq s_{r}
$$

for all $x, y \in M$;

- metrically proper ${ }^{8}$ if $f^{-1}(B)$ is bounded in $M$ for each bounded subset $B \subset M^{\prime}$

- uniformly metrically proper ${ }^{9}$ if, for each $r \geq 0$, there is some $t_{r} \geq 0$ so that

$$
d^{\prime}(f(x), f(y)) \leq r \Longrightarrow d(x, y) \leq t_{r}
$$

for all $x, y \in M$;

- coarse ${ }^{10}$ if it is uniformly expansive and metrically proper; and

- rough if it is uniformly expansive and uniformly metrically proper.

\footnotetext{
7 This name is taken from $[\mathbf{8 5}$. Other terms used to denote the same property are uniformly bornologous 84 and coarsely Lipschitz [20.

${ }^{8}$ This term is used in $\mathbf{8 5}$.

${ }^{9}$ This term is used in $\mathbf{8 5}$. Another term used to denote the same property is effectively proper 20 .

${ }^{10}$ This is a particular case of coarse maps between general coarse spaces $\mathbf{8 5}, \mathbf{6 5}$.
} 
If $f$ satisfies the conditions of uniform expansiveness and uniform metric properness with respective mappings $r \mapsto s_{r}$ and $r \mapsto t_{r}$ (simply denoted by $s_{r}$ and $t_{r}$ ), then $\left(s_{r}, t_{r}\right)$ is called a rough distortion of $f$; the term $\left(s_{r}, t_{r}\right)$-rough map may be also used. When $s_{r}=t_{r}$, we simply say that $s_{r}$ is a rough distortion of $f$, or $f$ is an $s_{r}$-rough map. If $f$ is a coarse map and there is a coarse map $g: M^{\prime} \rightarrow M$ such that $g f$ and $f g$ are close to $\operatorname{id}_{M}$ and $\mathrm{id}_{M^{\prime}}$, respectively, then $f$ is called a coarse equivalence. If $f$ is an $s_{r}$-rough map and there is an $s_{r}$-rough map $g: M^{\prime} \rightarrow M$ such that $g f$ and $f g$ are $c$-close to $\mathrm{id}_{M}$ and $\operatorname{id}_{M^{\prime}}$, respectively, then $f$ is called an $\left(s_{r}, c\right)$-rough equivalence, and $\left(s_{r}, c\right)$ is called a rough equivalence distortion of $f$. If there is a coarse (respectively, rough) equivalence $M \rightarrow M^{\prime}$, then $M$ and $M^{\prime}$ are coarsely (respectively, roughly ${ }^{11}$ equivalent. A coarse (respectively, rough) equivalence $M \rightarrow M$ is called a coarse (respectively, rough) transformation of $M$.

Two metrics $d_{1}$ and $d_{2}$ on the same set $S$ are called coarsely (respectively, roughly) equivalent if the identity map $\left(S, d_{1}\right) \rightarrow\left(S, d_{2}\right)$ is a coarse (respectively, rough) equivalence. When $S$ is equipped with a coarse (respectively, rough) equivalence class of metrics, it is called a metric coarse space ${ }^{12}$ (respectively, rough space). The metric coarse space and rough space induced by the metric space $M$ is denoted by $[M]$. The condition on a map $M \rightarrow M^{\prime}$ to be coarse (respectively, rough) depends only on the metric coarse spaces (respectively, rough spaces) $[M]$ and $\left[M^{\prime}\right]$. Any composition of coarse (respectively, rough) maps is (respectively, rough); more precisely, if $f: M \rightarrow M^{\prime}$ and $f^{\prime}: M^{\prime} \rightarrow M^{\prime \prime}$ are $s_{r}$-rough maps, then $f^{\prime} f$ is $s_{s_{r}}$-rough. Moreover the composite of coarse/rough maps is compatible with the closeness relation in an obvious sense. So the closeness classes of coarse (respectively, rough) maps between metric coarse spaces (respectively, rough spaces) form a category called metric coarse category ${ }^{13}$ (respectively, rough category). Thus rough equivalences are the maps that induce isomorphisms in the rough category. There are interesting differences between the rough category and the metric coarse category, cf. [85, but the following result shows that they have the same isomorphisms.

Proposition 2.36 (Álvarez-Candel [1, Proposition 3.8]). Any coarse equivalence between metric spaces is uniformly metrically proper. Moreover the definition of uniform metric properness is satisfied with constants that depend only on the constants involved in the definition of coarse equivalence.

Observe that, if $f: M \rightarrow M^{\prime}$ is an $\left(s_{r}, c\right)$-rough equivalence, then $f(M)$ is a $c$-net in $M^{\prime}$.

Proposition 2.37. If $f: M \rightarrow M^{\prime}$ is $s_{r}$-rough and $f(M)$ is a $K$-net in $M^{\prime}$, then $f$ is a $\left(\bar{s}_{r}, c\right)$-rough equivalence, where $\bar{s}_{r}=\max \left\{s_{r+2 K}, s_{r}+2 K\right\}$ and $c=$ $\max \left\{K, s_{K}\right\}$.

Proof. Let $g: M^{\prime} \rightarrow M$ be defined by choosing, for each $x^{\prime} \in M^{\prime}$, a point $g\left(x^{\prime}\right) \in M$ so that $d^{\prime}\left(x^{\prime}, f g\left(x^{\prime}\right)\right) \leq K$. Thus $f g$ is $K$-close to $\operatorname{id}_{M^{\prime}}$.

For $x \in M$, we have $d^{\prime}(f(x), f g f(x)) \leq K$, giving $d(x, g f(x)) \leq s_{K}$. Hence $g f$ is $s_{K}$-close to $\operatorname{id}_{M}$.

\footnotetext{
${ }^{11}$ The term uniform closeness is used in [20] when two metric spaces are roughly equivalent.

${ }^{12}$ This notion of metric coarse space is equivalent to the concept of coarse space induced by a metric 85, 86.

${ }^{13}$ This is a subcategory of the coarse category [85, 65.
} 
For $x^{\prime}, y^{\prime} \in M^{\prime}$ with $d^{\prime}\left(x^{\prime}, y^{\prime}\right) \leq r$, we have

$$
d\left(f g\left(x^{\prime}\right), f g\left(y^{\prime}\right)\right) \leq d\left(f g\left(x^{\prime}\right), x^{\prime}\right)+d^{\prime}\left(x^{\prime}, y^{\prime}\right)+d^{\prime}\left(y^{\prime}, f g\left(x^{\prime}\right) \leq r+2 K,\right.
$$

obtaining $d\left(g\left(x^{\prime}\right), g\left(y^{\prime}\right)\right) \leq s_{r+2 K}$. Thus $g$ is $s_{r+2 K}$-uniformly expansive.

Suppose now that $d\left(g\left(x^{\prime}\right), g\left(y^{\prime}\right)\right) \leq r$. Then

$$
d^{\prime}\left(x^{\prime}, y^{\prime}\right) \leq d^{\prime}\left(x^{\prime}, f g\left(x^{\prime}\right)\right)+d^{\prime}\left(f g\left(x^{\prime}\right), f g\left(y^{\prime}\right)\right)+d^{\prime}\left(f g\left(y^{\prime}\right), y^{\prime}\right) \leq s_{r}+2 K .
$$

So $g$ is also $\left(s_{r}+2 K\right)$-uniformly metrically proper.

The following is a direct consequence of Propositions 2.36 and 2.37

Corollary 2.38. For a map $f: M \rightarrow M^{\prime}$, the following conditions are equivalent:

(i) $f$ is rough.

(ii) $f: M \rightarrow f(M)$ is a rough equivalence.

(iii) $f: M \rightarrow f(M)$ is a coarse equivalence.

According to Corollary 2.38, coarse maps can be also properly called coarse embeddings ${ }^{14}$.

Proposition 2.39 (Álvarez-Candel [11, Proposition 3.13]). The following properties are true:

(1) Any $(\lambda, b)$-large scale Lipschitz map satisfies the condition of uniform expansiveness with $s_{r}=\lambda r+b$.

(2) Any $(\lambda, b, c)$-large scale Lipschitz equivalence is an $\left(s_{r}, c\right)$-rough equivalence, where $s_{r}=\lambda r+b+2 c$.

REMARK 2.40. In fact, the proof of Proposition 2.39 is elementary: (1) is obvious, and (2) follows from Lemma 2.21

Definition 2.41. A class of maps, $\left\{f_{i}: M_{i} \rightarrow M_{i}^{\prime}\right\}$, is said to be a class of:

- equi-uniformly expansive maps if they satisfy the condition of uniform expansiveness with the same mapping $r \mapsto s_{r}$.

- equi-metrically proper maps if they satisfy the condition of metric properness with the same mapping $r \mapsto t_{r}$.

- equi-rough maps (or equi-coarse embeddings) if they are rough with a common rough distortion; and

- equi-rough equivalences (or equi-coarse equivalences) if they are rough equivalences with a common rough equivalence distortion.

It is not possible to define "equi-coarse maps," but the concept of "equi-rough equivalences" makes sense according to Proposition 2.36 Given a family of sets $\left\{S_{i}\right\}$, and metrics $d_{i, 1}$ and $d_{i, 2}$ on each $S_{i}$, it is said that $\left\{d_{i, 1}\right\}$ is equi-roughly equivalent (or equi-coarsely equivalent) to $\left\{d_{i, 2}\right\}$ if the identity maps $\left(S_{i}, d_{i, 1}\right) \rightarrow$ $\left(S_{i}, d_{i, 2}\right)$ are equi-rough equivalences.

EXAMPLE 2.42. If the metric spaces $M_{i}$ are bounded, they are equi-coarsely equivalent to the singleton metric space if and only $\sup _{i} \operatorname{diam} M_{i}<\infty$.

Remark 2.43. (i) By Proposition 2.37, families of equi-rough maps whose images are equi-nets are equi-rough equivalences.

${ }^{14}$ This concept is generalized to arbitrary coarse spaces as maps that define a coarse equivalence to their image [86 Section 11.1]. 
(ii) By Proposition 2.39, families of equi-large scale Lipschitz maps are equiuniformly expansive, and families of equi-large scale Lipschitz equivalences are equi-rough equivalences.

There are rough equivalences that are not large scale Lipschitz equivalences, cf. [11, Example 3.14].

Proposition 2.44. If there are disjoint unions, $M=\cup_{i=0}^{\infty} M_{i}$ and $M^{\prime}=\bigcup_{i=0}^{\infty} M_{i}^{\prime}$, such that:

- $M_{i}$ and $M_{i}^{\prime}$ are bounded for all $i$;

- $\min _{i<j} d\left(M_{i}, M_{j}\right) \rightarrow \infty$ and $\min _{i<j} d\left(M_{i}^{\prime}, M_{j}^{\prime}\right) \rightarrow \infty$ as $j \rightarrow \infty$ and

- $\left\{M_{i}\right\}$ are equi-coarsely equivalent to $\left\{M_{i}^{\prime}\right\}$;

then $M$ is coarsely equivalent to $M^{\prime}$.

Proof. There are sequences $u_{i}, v_{i} \rightarrow \infty$ such that:

- $d\left(M_{i}, M_{j}\right) \geq u_{j}$ and $d\left(M_{i}^{\prime}, M_{j}^{\prime}\right) \geq u_{j}$ if $i<j$; and

- $\operatorname{diam}\left(\cup_{i \leq j} M_{i}\right) \leq v_{j}$ and $\operatorname{diam}\left(\cup_{i \leq j} M_{i}^{\prime}\right) \leq v_{j}$ for all $j$.

Moreover there are rough equivalences $f_{i}: M_{i} \rightarrow M_{i}^{\prime}$ with a common rough equivalence distortion $\left(s_{r}, c\right)$. Thus there are $s_{r}$-rough maps $g_{i}: M_{i}^{\prime} \rightarrow M_{i}$ such that $g_{i} f_{i}$ and $f_{i} g_{i}$ are $c$-close to $\operatorname{id}_{M_{i}}$ and $\operatorname{id}_{M_{i}^{\prime}}$, respectively. Since the sets $M_{i}$ are disjoint from each other, the maps $f_{i}$ can be combined to define a map $f: M \rightarrow M^{\prime}$. Similarly, the maps $g_{i}$ can be also combined to define a map $g: M^{\prime} \rightarrow M$.

Clearly, $g f$ and $f g$ are $c$-close to $\mathrm{id}_{M}$ and $\operatorname{id}_{M^{\prime}}$, respectively. Thus it only remains to prove that $f$ and $g$ are rough.

For $r \geq 0$, let

$$
\bar{s}_{r}=\max \left\{s_{r}, v_{j} \mid u_{j} \leq r\right\} .
$$

Suppose that $d(x, y) \leq r$ for some $x, y \in M$ and $r \geq 0$. If $x, y \in M_{i}$ for some $i$, then $d^{\prime}(f(x), f(y)) \leq s_{r} \leq \bar{s}_{r}$. If $x \in M_{i}$ and $y \in M_{j}$ for some $i<j$, then $u_{j} \leq d(x, y) \leq r$, and therefore $d^{\prime}(f(x), f(y)) \leq v_{j} \leq \bar{s}_{r}$.

Now, suppose that $d(f(x), f(y)) \leq r$ for some $x, y \in M$ and $r \geq 0$. If $x, y \in M_{i}^{\prime}$ for some $i$, then $d(x, y) \leq s_{r} \leq \bar{s}_{r}$. If $x \in M_{i}$ and $y \in M_{j}$ for some $i<j$, then $u_{j} \leq d^{\prime}(f(x), f(y)) \leq r$, and therefore $d(x, y) \leq v_{j} \leq \bar{s}_{r}$. Thus $f$ is $\bar{s}_{r}$-rough. In the same way, we get that $g$ is $\bar{s}_{r}$-rough. 



\section{CHAPTER 3}

\section{Some classes of metric spaces}

In this chapter, we recall some classes of metric spaces: graphs, metric spaces of coarse bounded geometry, and coarsely quasi-convex metric spaces. They contain the coarse quasi-isometric types of leaves of compact foliated spaces. We also introduce the class of coarsely quasi-symmetric metric spaces, which plays a relevant role in one of our main results, Theorem 1.3 .

\subsection{Graphs}

Suppose that $M$ is the set of vertices of a connected graph $G$, equipped with the metric $d$ defined by setting $d(x, y)$ equal to the minimum number of consecutive edges of $M$ needed to joint $x$ and $y$ (being 0 if $x=y$ ). When equipped with this metric, $M$ is called the metric space of vertices of the connected graph $G$. Observe that $M$ (equipped with $d$ ) and $G$ determine each other. Since $d$ has values in $\mathbb{N}$, it will be enough to consider (open, closed) $r$-balls, $r$-penumbras, $r$-boundaries, $K$-nets and $\delta$-separated sets with $r, K, \delta \in \mathbb{N}$.

ExAmple 3.1. Let $\Gamma$ be a group and let $S$ be a generating set for $\Gamma$. The Cayley graph of $\Gamma$ relative to $S, G=G(\Gamma, S)$, is the graph that has one vertex for each element of $\Gamma$ and one edge joining $\gamma_{1}$ and $\gamma_{2}$ if $\gamma_{1} \gamma_{2}^{-1} \in S \cup S^{-1}$. The vertex set of $G$ is the set of elements of $\Gamma$, and the metric induced on $\Gamma$ is the word metric relative to $S$, denoted by $d_{S}$. For every $\gamma \in \Gamma$, let $|\gamma|$ denote its length ${ }^{1}$ with respect to $S$. Then $d_{S}\left(\gamma_{1}, \gamma_{2}\right)=\left|\gamma_{1} \gamma_{2}^{-1}\right|$. It is well known that if $\Gamma$ is finitely generated then all those metrics that are induced by finite generating sets for $\Gamma$ are in the same Lipschitz class. The group operation of $\Gamma$ induces an action on the right ${ }^{2}$ of $\Gamma$ on $G$, and the metric $d_{S}$ is invariant under this action: $d_{S}\left(\gamma_{1} \gamma, \gamma_{2} \gamma\right)=d_{S}\left(\gamma_{1}, \gamma_{2}\right)$.

EXAmPle 3.2. With the notation of Example 3.1. if $\Gamma_{0}$ is a subgroup of $\Gamma$ (not necessarily a normal subgroup), the right invariant graph structure and metric $d_{S}$ on $\Gamma$ induces a graph structure and the corresponding metric on the homogeneous space of right cosets $\Gamma / \Gamma_{0}$. This graph is called Schreier coset graph.

EXAmPle 3.3. Let $\Gamma$ be a group. Let $S$ be a generating set for $\Gamma$ and let $d_{S}$ be the right invariant metric induced by $S$ on $\Gamma$.

Let $\Gamma$ act on the left on a space $X$ and denote the action by $x \mapsto \gamma x$. There is a natural bijection between the orbit, $\Gamma(x)$ of a point $x$, consisting of all the points $\gamma(x), \gamma \in \Gamma$, and the space of right cosets of $\Gamma$ relative to the subgroup of $\Gamma$ that fixes $x$ : If $\Gamma_{x}$ is the set of $\gamma \in \Gamma$ such that $x \gamma=x$, then the assignment $[\gamma] \in \Gamma / \Gamma_{x} \mapsto \gamma x$

\footnotetext{
${ }^{1}$ The minimum number of elements in $S \cup S^{-1}$ whose product is $\gamma$.

${ }^{2}$ The definition of Cayley graph can be modified so that left translations are isometries, by declaring that the existence one edge joining $\gamma_{1}$ and $\gamma_{2}$ means $\gamma_{1}^{-1} \gamma_{2} \in S \cup S^{-1}$.
} 
is independent of the representative of the class $[\gamma]$, for if $\gamma^{\prime} \in[\gamma]$, then $\gamma^{-1} \gamma^{\prime}$ fixes $x$, so $\gamma^{\prime} x=\gamma x$.

The metric $d_{S}$ induced by $S$ on $\Gamma$ in turn induces a metric on the orbit $\Gamma(x)$. This metric is actually independent of the chosen point in the orbit: if $y \in X$ is in the same orbit as $x$, then $\gamma_{y} x=y$ for some $\gamma_{y} \in \Gamma$. The stabilizer subgroup of $y$ is related to that of $x$ via the conjugation $\Gamma_{y} \gamma_{y}=\gamma_{y} \Gamma_{x}$, and so the homogeneous spaces $\Gamma / \Gamma_{y}$ and $\Gamma / \Gamma_{x}$ are isometric (with the metric induced by $d_{S}$ ) via right multiplication by $\gamma_{y}$.

The reverse inclusion of 2.2 also holds with natural numbers ${ }^{3}$

$$
\operatorname{Pen}(S, r+s)=\operatorname{Pen}(\operatorname{Pen}(S, r), s)
$$

for $S \subset M$ and $r, s \in \mathbb{N}$; more precisely,

$$
\operatorname{Pen}(S, r+s) \backslash S=\operatorname{Pen}(\operatorname{Pen}(S, r) \backslash S, s) \backslash S .
$$

Note that (3.1) follows from $(3.2$ and 2.2 . The inclusion " $\supset$ " of 3.2 is given by 2.2. To prove the reverse inclusion, assume that $r, s>0$ (if one of $r=0$ or $s=0$ there is nothing to prove). If $x \in \operatorname{Pen}(S, r+s) \backslash S$, then there is a finite sequence $z_{0}, z_{1}, \ldots, z_{k}=x$ in $M$ with $z_{0} \in S, k \leq r+s$, and $d\left(z_{l-1}, z_{l}\right)=1$ for all $l \in\{1, \ldots, k\}$, and furthermore that $z_{l} \in M \backslash S$ for $l \geq 1$. If $k \leq r$, then $x \in \operatorname{Pen}(S, r) \backslash S \subset$ $\operatorname{Pen}(\operatorname{Pen}(S, r) \backslash S, s) \backslash S$; and if $k>r$, then $z_{r} \in \operatorname{Pen}(S, r)$ and $d\left(x, z_{r}\right) \leq k-r \leq s$. This implies that $x \in \operatorname{Pen}(\operatorname{Pen}(S, r) \backslash S, s) \backslash S$, which concludes the proof of 3.2 .

Lemma 3.4. Let $A$ be a $K$-net in $M$ for some $K \in \mathbb{N}$. Then, for every $S \subset M$ and all natural $r \geq K$, the set $\operatorname{Pen}(S, r) \cap A$ is a $2 K$-net in $\operatorname{Pen}(S, r)$; in particular, for every $x \in M, \bar{B}(x, r) \cap A$ is a $2 K$-net in $\bar{B}(x, r)$.

Proof. For each $x \in \operatorname{Pen}(S, r)$ there is $y \in \operatorname{Pen}(S, r-K)$ with $d(x, y) \leq K$ by (3.1), and there is $z \in A$ with $d(y, z) \leq K$ since $A$ is a $K$-net. So $z \in \operatorname{Pen}(S, r) \cap A$ by $(3.1)$, and $d(x, z) \leq 2 K$ by the triangle inequality.

Recall that the degree (or valence) of a vertex $x$ of $G$ is the number of edges that meet at $x$, which is denoted by $\operatorname{deg}(x)=\operatorname{deg}_{G}(x)$. Suppose from now on that $G$ is of finite type in the sense that there is some $K \in \mathbb{N}$ such that each vertex is of degree $\leq K$, and assume that $K \geq 2$ (if $K=0$, then $G$ has just one vertex and no edges; if $K=1$, then $G$ has at most two vertices and one edge joining them). For each $r \in \mathbb{N}$, let

$$
\Lambda_{K, r}= \begin{cases}1+K \frac{(K-1)^{r}-1}{K-2} & \text { if } K>2 \\ 1+2 r & \text { if } K=2\end{cases}
$$

Then

$$
|\bar{B}(x, r)| \leq 1+K+K(K-1)+\cdots+K(K-1)^{r-1}=\Lambda_{K, r}
$$

for all $x \in M$ and $r \in \mathbb{N}$. Therefore

$$
|\operatorname{Pen}(S, r)| \leq \Lambda_{K, r}|S|
$$

for any $S \subset M$ and $r \in \mathbb{N}$.

The growth type of the function $r \mapsto|B(x, r)|(r \geq 1)$ is independent of the choice of $x \in M$, and is called the growth type of $M$ (as set of vertices of a connected graph 4 , or of $G$ (as graph).

\footnotetext{
${ }^{3}$ On complete path metric spaces, these equality holds for all $r, s \geq 0$.

${ }^{4}$ This definition is indeed valid for any metric space with finite balls.
} 
The boundary of a subset $S \subset M$ is $\partial S=\partial_{1} S$. The sets $\partial S \cap S$ and $\partial S \backslash S$ are respectively called inner and outer boundaries. Since

$$
\partial S \cap S \subset \operatorname{Pen}(\partial S \backslash S, 1) \text {, and } \partial S \backslash S \subset \operatorname{Pen}(\partial S \cap S, 1),
$$

it follows by (3.5) that

$$
\frac{1}{\Lambda_{K, 1}}|\partial S \backslash S| \leq|\partial S \cap S| \leq \Lambda_{K, 1}|\partial S \backslash S| .
$$

Lemma 3.5. $\partial_{r} S=\operatorname{Pen}(\partial S, r-1)$ for all $r \in \mathbb{Z}^{+}$.

Proof. By 3.2 ,

$$
\begin{aligned}
\partial_{r} S= & \operatorname{Pen}(S, r) \cap \operatorname{Pen}(M \backslash S, r) \\
= & ((\operatorname{Pen}(S, r) \cap \operatorname{Pen}(M \backslash S, r)) \backslash S) \\
& \cup(\operatorname{Pen}(S, r) \cap \operatorname{Pen}(M \backslash S, r) \cap S) \\
= & (\operatorname{Pen}(S, r) \backslash S) \cup(\operatorname{Pen}(M \backslash S, r) \cap S) \\
= & (\operatorname{Pen}(\operatorname{Pen}(S, 1) \backslash S, r-1) \backslash S) \\
& \cup(\operatorname{Pen}(\operatorname{Pen}(M \backslash S, 1) \cap S, r-1) \cap S) \\
= & (\operatorname{Pen}(\partial S \backslash S, r-1) \backslash S) \cup(\operatorname{Pen}(\partial S \cap S, r-1) \cap S) \\
\subset & \operatorname{Pen}(\partial S, r-1) .
\end{aligned}
$$

On the other hand, by (2.1) and (3.1),

$$
\begin{aligned}
\operatorname{Pen}(\partial S, r-1) & =\operatorname{Pen}(\operatorname{Pen}(S, 1) \cap \operatorname{Pen}(M \backslash S, 1), r-1) \\
\subset \operatorname{Pen}(\operatorname{Pen}(S, 1), r-1) \cap \operatorname{Pen}(\operatorname{Pen}(M \backslash S, 1), r-1) & =\operatorname{Pen}(S, r) \cap \operatorname{Pen}(M \backslash S, r)=\partial_{r} S .
\end{aligned}
$$

Lemma 3.5 and 3.5 give

$$
\left|\partial_{r} S\right| \leq \Lambda_{K, r-1}|\partial S|
$$

The metric space $M$ (as set of vertices of a connected graph) is called Følner if it contains a sequence of finite subsets $S_{n}$ such that $\left|\partial S_{n}\right| /\left|S_{n}\right| \rightarrow 0$ as $n \rightarrow \infty$.

Let $\left\{G_{i}\right\}$ be a class of connected graphs, and let $\left\{M_{i}\right\}$ be the class of metric spaces defined by their vertices. Then $\left\{G_{i}\right\}$ is said to be of equi-finite type if there is $K \in \mathbb{N}$ such that each vertex at each $G_{i}$ meets at most $K$ edges. The class $\left\{M_{i}\right\}$ is called equi-Følner if each $M_{i}$ has a Følner sequence $S_{i, n}$ such that, for some $a>0$, $\left|\partial S_{i, n}\right| /\left|S_{i, n}\right| \leq a\left|\partial S_{j, n}\right| /\left|S_{j, n}\right|$ for all $i$ and $j$.

\subsection{Metric spaces of coarse bounded geometry}

Definition 3.6 (Block-Weinberger [20]). A quasi-lattice $\Gamma$ of $M$ is an $R$-net of $M$ for some $R \geq 0$ such that $|\Gamma \cap \bar{B}(x, r)| \leq Q_{r}$ for every $x \in M$, where $r \mapsto Q_{r}$ $\left(r, Q_{r} \geq 0\right)$ is a mapping independent of $x$; the term $\left(R, Q_{r}\right)$-quasi-lattice may be also used. It is said that $M$ is of coarse bounded geometry if it has an $\left(R, Q_{r}\right)$ quasi-lattice for some $\left(R, Q_{r}\right)$; in this case, $\left(R, Q_{r}\right)$ is called a coarse bound of $M$. 
EXAMPLE 3.7 (Block-Weinberger [20]). (i) If $M$ is the metric space of vertices of any connected graph $G$, then $M$ is of coarse bounded geometry if and only if $G$ is of finite type; indeed, if each vertex meets at most $K$ edges, then $M$ is a $\left(0, \Lambda_{K, r}\right)$-quasi-lattice in itself by (3.4), where $\Lambda_{K, r}$ is given by 3.3 .

(ii) If $M$ is a connected complete Riemannian manifold with a positive invectivity radius and whose Ricci curvature is bounded from below, then it is of coarse bounded geometry. Recall that a Riemannian manifold is said to be of bounded geometry if it has a positive injectivity radius and each covariant derivative of arbitrary order of its curvature tensor is uniformly bounded. Thus $M$ is of coarse bounded geometry if it is of bounded geometry.

Definition 3.8. The class $\left\{M_{i}\right\}$ is said to be of equi-coarse bounded geometry when the metric spaces $M_{i}$ are of coarse bounded geometry with a common coarse bound $\left(R, Q_{r}\right)$. In this case, a class $\left\{\Gamma_{i} \subset M_{i}\right\}$ of $\left(R, Q_{r}\right)$-quasi-lattices is called a class of equi-quasi-lattices.

EXAMPLE 3.9. If $\left\{M_{i}\right\}$ is the class of metric spaces of vertices of a class of corresponding connected graphs $\left\{G_{i}\right\}$, then $\left\{M_{i}\right\}$ is of equi-coarse bounded geometry if and only if $\left\{G_{i}\right\}$ is of equi-finite type. If a class of connected complete Riemannian manifolds have injectivity radius bounded from below by a common positive constant, and Ricci curvature uniformly bounded from below by a common constant, then they are of equi-coarse bounded geometry.

Proposition 3.10. Suppose that $\Gamma$ is an $\left(R, Q_{r}\right)$-quasi-lattice of $M, f: M \rightarrow$ $M^{\prime}$ is an $\left(s_{r}, t_{r}\right)$-rough map, and that there is a rough map $g: M^{\prime} \rightarrow M$ such that $g f$ and $f g$ are c-close to $\operatorname{id}_{M}$ and $\operatorname{id}_{M^{\prime}}$, respectively. Then $\Gamma^{\prime}=f(\Gamma)$ is an $\left(R^{\prime}, Q_{r}^{\prime}\right)$-quasi-lattice of $M^{\prime}$, with $R^{\prime}=c+s_{R}$ and $Q_{r}^{\prime}=Q_{t_{r+R^{\prime}}}$.

Proof. For each $x^{\prime} \in M^{\prime}$, there is $y \in \Gamma$ such that $d\left(g\left(x^{\prime}\right), y\right) \leq R$. Then $y^{\prime}=f(y) \in \Gamma^{\prime}$ and

$$
d^{\prime}\left(x^{\prime}, y^{\prime}\right) \leq d^{\prime}\left(x^{\prime}, f g\left(x^{\prime}\right)\right)+d^{\prime}\left(f g\left(x^{\prime}\right), y^{\prime}\right) \leq c+s_{R} .
$$

Moreover

$$
\Gamma^{\prime} \cap \bar{B}_{M^{\prime}}\left(x^{\prime}, r\right) \subset \bar{B}_{\Gamma^{\prime}}\left(y^{\prime}, r+R^{\prime}\right) \subset f\left(\bar{B}_{\Gamma}\left(y, t_{r+R^{\prime}}\right)\right)
$$

for all $r \geq 0$, obtaining

$$
\left|\Gamma^{\prime} \cap \bar{B}_{M^{\prime}}\left(x^{\prime}, r\right)\right| \leq\left|\Gamma \cap \bar{B}_{M}\left(y, t_{r+R^{\prime}}\right)\right| \leq Q_{t_{r+R^{\prime}}} .
$$

Corollary 3.11. If $\Gamma$ is an $\left(R, Q_{r}\right)$-quasi-lattice of $M$, and $\phi: M \rightarrow M^{\prime}$ is a $(\lambda, b, c)$-large scale Lipschitz equivalence, then $\Gamma^{\prime}=\phi(\Gamma)$ is an $\left(R^{\prime}, Q_{r}^{\prime}\right)$-quasi-lattice of $M^{\prime}$, where $R^{\prime}=\lambda R+b+c$ and $Q_{r}^{\prime}=Q_{\lambda\left(r+R^{\prime}\right)+b+2 c}$.

Proof. By Proposition 2.39, we can apply Proposition 3.10 with $s_{r}=\lambda r+b$ and $t_{r}=\lambda r+b+2 c$.

COROllary 3.12. If $M$ is of coarse bounded geometry with coarse bound $\left(R, Q_{r}\right)$, and there is a $(K, C)$-coarse quasi-isometry of $M$ to $M^{\prime}$, then $M^{\prime}$ is of coarse bounded geometry with coarse bound $\left(R^{\prime}, Q_{r}^{\prime}\right)$, where

$$
R^{\prime}=K+C R+2 C K, \quad Q_{r}^{\prime}=Q_{C\left(r+R^{\prime}\right)+2 C K+2 K} .
$$


Proof. By Proposition 2.26, there is a $(C, 2 C K, K)$-large scale Lipschitz equivalence $\phi: M \rightarrow M^{\prime}$. Let $\Gamma$ be an $\left(R, Q_{r}\right)$-quasi-lattice of $M$. Then $\phi(\Gamma)$ is an $\left(R^{\prime}, Q_{r}^{\prime}\right)$-quasi-lattice of $M^{\prime}$ by Corollary 3.11

Remark 3.13. According to Corollary 3.12 , equi-coarse bounded geometry is preserved by equi-coarse quasi-isometries.

REMARK 3.14. Another version of Corollary 3.12, with $R^{\prime}=6 C \max \{R, K\}$ and $Q_{r}^{\prime}=Q_{C\left(r+R^{\prime}\right)}$, can be proved without passing to large scale equivalences.

\subsection{Coarsely quasi-symmetric metric spaces}

DEFINITION 3.15. Let $\mathcal{T}$ be a set of coarsely quasi-isometric transformations of $M$, let $\Phi$ be a set of maps $M \rightarrow M$, and let $R \geq 0$. The set $\mathcal{T}$ is called:

- transitive when, for all $x, y \in M$, there is some $f \in \mathcal{T}$ such that $x \in \operatorname{dom} f$, $y \in \operatorname{im} f$ and $f(x)=y$; and

- R-quasi-transitive if, for all $x, y \in M$, there is some $f \in \mathcal{T}$ and $z \in \operatorname{dom} f$ such that $d(x, z) \leq R$ and $d(f(z), y) \leq R$.

The set $\Phi$ is called:

- transitive when, for all $x, y \in M$, there is some $\phi \in \Phi$ with $\phi(x)=y$; and

- R-quasi-transitive if, for all $x, y \in M$, some $\phi \in \Phi$ satisfies $d(f(x), y) \leq R$.

Definition 3.16. A metric space $M$ is called coarsely quasi-symmetric if there is a transitive set of equi-coarsely quasi-isometric transformations of $M$.

Definition 3.17. A class $\left\{M_{i}\right\}$ is called equi-coarsely quasi-symmetric if, for some $K \geq 0$ and $C \geq 1$, there is a transitive class of $(K, C)$-coarsely quasi-isometric transformations of every $M_{i}$.

LEMma 3.18. The following statements are equivalent:

(i) $\left\{M_{i}\right\}$ is equi-coarsely quasi-symmetric.

(ii) For some $R, K \geq 0$ and $C \geq 1$, there is an $R$-quasi-transitive class of $(K, C)$ quasi-isometric transformations of each $M_{i}$.

(iii) For some $\lambda \geq 1$ and $b, c \geq 0$, there is a transitive class of $(\lambda, b, c)$-large scale Lipschitz transformations of each $M_{i}$.

(iv) For some $R, b, c \geq 0$ and $\lambda \geq 1$, there is an $R$-quasi-transitive class of $(\lambda, b, c)$ large scale Lipschitz transformations of each $M_{i}$.

Proof. This follows from Propositions 2.26, 2.27 and 2.31, and Corollary 2.33,

Proposition 3.19. (Equi-)coarse quasi-symmetry is preserved by (equi-)coarse quasi-isometries.

Proof. Assume that there is some transitive set $\Phi$ of $(\lambda, b, c)$-large scale Lipschitz transformations of $M$, and there is a $(\lambda, b, c)$-large scale Lipschitz equivalence $\xi: M \rightarrow M^{\prime}$. Let $\zeta: M^{\prime} \rightarrow M$ be a $(\lambda, b)$-large scale Lipschitz map so that $\zeta \xi$ and $\xi \zeta$ are $c$-close to $\mathrm{id}_{M}$ and $\mathrm{id}_{M^{\prime}}$, respectively. By Lemma 2.24 , it follows that $\Phi^{\prime}:=\{\xi \phi \zeta \mid \phi \in \Phi\}$ is a family of $\left(\lambda^{\prime}, b^{\prime}, c^{\prime}\right)$-large scale Lipschitz transformations of $M^{\prime}$, where $\left(\lambda^{\prime}, b^{\prime}, c^{\prime}\right)$ depends only on $(\lambda, b, c)$. For all $x^{\prime}, y^{\prime} \in M^{\prime}$, there is some 
$\phi \in \Phi$ such that $\phi \zeta\left(x^{\prime}\right)=\zeta\left(y^{\prime}\right)$. Thus $\phi^{\prime}:=\xi \phi \zeta \in \Phi^{\prime}$ satisfies

$$
\begin{aligned}
d^{\prime}\left(\phi^{\prime}\left(x^{\prime}\right), y^{\prime}\right) \leq d^{\prime}\left(\phi^{\prime}\left(x^{\prime}\right), \xi \zeta\left(y^{\prime}\right)\right)+d^{\prime}\left(\zeta \xi\left(y^{\prime}\right), y^{\prime}\right) & \\
& \leq \lambda d\left(\phi \zeta\left(x^{\prime}\right), \zeta\left(y^{\prime}\right)\right)+b+c=b+c,
\end{aligned}
$$

obtaining that $\Phi^{\prime}$ is $(b+c)$-quasi-transitive. Hence the result follows from Lemma 3.18 and Proposition 2.26 .

REMARK 3.20. A more involved proof can be given by using coarse composites of coarse quasi-isometries, whose coarse distortion is controlled by Proposition 2.12 .

\subsection{Coarsely quasi-convex metric spaces}

Definition 3.21. A metric space, $M$, is said to be coarsely quasi-convex if there are $a, b, c \geq 0$ such that, for each $x, y \in M$, there exists a finite sequence of points $x=x_{0}, \ldots, x_{n}=y$ in $M$ such that $d\left(x_{k-1}, x_{k}\right) \leq c$ for all $k \in\{1, \ldots, n\}$, and

$$
\sum_{k=1}^{n} d\left(x_{k-1}, x_{k}\right) \leq a d(x, y)+b .
$$

A class of metric spaces is said to be equi-coarsely quasi-convex if all of them satisfy this condition with the same constants $a, b$, and $c$.

REMARK 3.22. Coarse quasi-convexity is a coarsely quasi-isometric version of the following condition introduced by Gromov: For each $x, y \in M$ and $\varepsilon>0$, there is some $z \in M$ such that

$$
\max \{d(x, z), d(y, z)\}<\frac{1}{2} d(x, y)+\varepsilon .
$$

This property may be called approximate convexity because a subset of $\mathbf{R}^{n}$ satisfies it precisely when said subset has a convex closure. Gromov has shown that a complete metric space is a path metric space if and only if it is approximately convex [56, Theorem 1.8].

REMARK 3.23. The property of coarse quasi-convexity is slightly weaker than the property of monogenicity for coarse spaces defined by metrics [86] (monogenicity means that the condition of Definition 3.21 is satisfied with $a=1$ and $b=0$ ).

EXAMPLE 3.24. Any class of metric spaces, each being the space of vertices of a connected graph, is equi-coarsely quasi-convex (they satisfy the condition of Definition 3.21 with $a=1, b=0$ and $c=1$ ). Of course, any class of connected complete Riemannian manifolds is also coarsely quasi-convex since they are path metric spaces.

Proposition 3.25 (Álvarez-Candel 11, Theorem 3.11]). A metric space, $M$, is coarsely quasi-convex if and only if there exists a coarse quasi-isometry of $M$ to the metric space of vertices of some connected graph. A class, $\left\{M_{i}\right\}$, is equi-coarsely quasi-convex if and only if $\left\{M_{i}\right\}$ is equi-coarsely quasi-isometric to a family of metric spaces of vertices of connected graphs.

REMARK 3.26. In [11, Theorem 3.11], the result was stated using complete path metric spaces instead of graphs, but indeed a graph is constructed in its proof.

REMARK 3.27. Proposition 3.25 is a coarsely quasi-isometric version of [86, Proposition 2.57], which asserts that the monogenic coarse structures are those that are coarsely equivalent to path metric spaces. 
REMARK 3.28. As a consequence of Proposition 3.25, we get that (equi-) coarse quasi-convexity is invariant by (equi-) coarse quasi-isometries.

Proposition 3.29 (Álvarez-Candel [11, Proposition 3.15]). (Equi-)uniformly expansive maps with (equi-) coarsely convex domains are (equi-) large scale Lipschitz.

COROLlary 3.30 (Álvarez-Candel [11, Corollary 3.16]). (Equi-)coarse equivalences with (equi-) coarsely convex domains are (equi-)large scale Lipschitz equivalences. 



\section{CHAPTER 4}

\section{Growth of metric spaces}

In this chapter, we recall or introduce the concepts and properties about growth on metric spaces that are needed to show some of our main results, Theorems 1.5 1.8 and Corollary 1.9 . Specially relevant is the role played by growth symmetry in Theorem 1.7 .

\subsection{Growth of non-decreasing functions}

Given non-decreasing functions $\unlhd^{1} u, v: \mathbb{R}^{+} \rightarrow \mathbb{R}^{+}$, the function $u$ is said to be dominated by the function $v$, written $u \leqslant v$, if there are $a, b \geq 1$ and $c>0$ such that $u(r) \leq a v(b r)$ for all $r \geq c$. For all $a, b \geq 1, c>0$ and $e \geq 0$, we have

$$
\begin{aligned}
b^{\prime}>b \quad \& \quad u(r) \leq a v(b r+e) \forall r & \geq c \\
& \Longrightarrow u(r) \leq a v\left(b^{\prime} r\right) \forall r \geq \max \left\{c, \frac{e}{b^{\prime}-b}\right\} .
\end{aligned}
$$

If $u \leqslant v \leqslant u$, then $u$ and $v$ represent the same growth type (or growth class) or have equivalent growth; this is an equivalence relation and "ミ" defines a partial order relation between growth types called domination. The growth type of $u$ may be denoted by $\operatorname{gr}(u)$, and we may write $\operatorname{gr}(u) \leq \operatorname{gr}(v)$ when $u \leqslant v$; then $\operatorname{gr}(u)<\operatorname{gr}(v)$ has the obvious meaning. For a class of pairs of non-decreasing functions $\mathbb{R}^{+} \rightarrow$ $\mathbb{R}^{+}$, equi-domination means that all of those pairs satisfy the above condition of domination with the same constants $a, b$ and $c$. A class of non-decreasing functions $\mathbb{R}^{+} \rightarrow \mathbb{R}^{+}$will be said to have equi-equivalent growth if they equi-dominate one another.

For non-decreasing functions $u, v: \mathbb{R}^{+} \rightarrow \mathbb{R}^{+}$, and constants $a, b \geq 1$ and $c>0$, if $u(r) \leq a v(b r)$ for all $r \geq c$, then

$$
\begin{gathered}
\limsup _{r \rightarrow \infty} \frac{\log u(r)}{\log r} \leq \limsup _{r \rightarrow \infty} \frac{\log v(r)}{\log r}, \\
\liminf _{r \rightarrow \infty} \frac{\log u(r)}{\log r} \leq \liminf _{r \rightarrow \infty} \frac{\log v(r)}{\log r}, \\
\liminf _{r \rightarrow \infty} \frac{\log u(r)}{r} \leq b \liminf _{r \rightarrow \infty} \frac{\log v(r)}{r}, \\
\limsup _{r \rightarrow \infty} \frac{\log u(r)}{r} \leq b \limsup _{r \rightarrow \infty} \frac{\log v(r)}{r} .
\end{gathered}
$$

Thus it makes sense to say that the growth type of $u$ is:

- exactly polynomial of degree $d \in \mathbb{N}$ if it is the growth type of the function $r \mapsto r^{d}$

\footnotetext{
${ }^{1}$ The usual definition of growth type uses functions $\mathbb{Z}^{+} \rightarrow \mathbb{R}^{+}$, but functions $\mathbb{R}^{+} \rightarrow \mathbb{R}^{+}$give rise to an equivalent concept.
} 
- polynomial if it is dominated by a polynomial growth of some exact degree; and

- exponential if it is the growth type of the function $r \mapsto e^{r}$, which is the same as the growth of $r \mapsto a^{r}$ for any $a>1$.

Observe that the growth type of $u$ is:

- polynomial if and only if $\limsup _{r \rightarrow \infty} \frac{\log u(r)}{\log r}<\infty$; and

- exponential if and only if $0<\liminf _{r \rightarrow \infty} \frac{\log u(r)}{r}<\infty$.

It is also said that the growth type of $u$ is:

- quasi-polynomia $2^{2}$ if $\lim \sup _{r \rightarrow \infty} \frac{\log u(r)}{r} \leq 0$;

- quasi-exponential if $0<\lim \sup _{r \rightarrow \infty} \frac{\log u(r)}{r}<\infty$; and

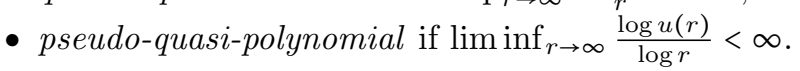

\subsection{Growth of metric spaces}

Suppose that $M$ and $M^{\prime}$ are of coarse bounded geometry, and $\left\{M_{i}\right\}$ and $\left\{M_{i}^{\prime}\right\}$ are of equi-coarse bounded geometry.

For a quasi-lattice $\Gamma$ of $M$ and $x \in \Gamma$, the function $r \mapsto v_{\Gamma}(x, r)=\left|B_{\Gamma}(x, r)\right|$ $(r \geq 1)$ is called the growth function of $M$ induced by $\Gamma$ and $x$.

Proposition 4.1. For $k \in\{1,2\}$, let $\Gamma_{k}$ be an $\left(R_{k}, Q_{r}^{k}\right)$-quasi-lattice of $M$, and $x_{k} \in \Gamma_{k}$. Take any $\delta \geq d\left(x_{1}, x_{2}\right)$. Then, for all $r \geq 1$,

$$
v_{\Gamma_{1}}\left(x_{1}, r\right) \leq Q_{R_{2}}^{1} v_{\Gamma_{2}}\left(x_{2}, r+\delta+R_{2}\right) .
$$

Proof. Since $B_{M}\left(x_{1}, r\right) \subset B_{M}\left(x_{2}, r+\delta\right)$ for all $r \geq 1$, and $\Gamma_{2}$ is an $R_{2}$-net, then

$$
B_{\Gamma_{1}}\left(x_{1}, r\right) \subset \underset{y \in B_{\Gamma_{2}}\left(x_{2}, r+\delta+R_{2}\right)}{\bigcup} \bar{B}_{M}\left(y, R_{2}\right) \cap \Gamma_{1},
$$

which implies the stated inequality.

The following definitions are justified by Proposition 4.1 .

Definition 4.2. The growth type (or growth class) of $M$ is the growth type of $r \mapsto v_{\Gamma}(x, r)$ for any quasi-lattice $\Gamma$ of $M$ and $x \in \Gamma$. We may also say that $M$ and $M^{\prime}$ have equivalent growth when they have the same growth type. The notation $\operatorname{gr}(M)$ may be used for the growth type of $M$.

Definition 4.3. Two classes of metric spaces, $\left\{M_{i}\right\}$ and $\left\{M_{i}^{\prime}\right\}$, have equiequivalent growth when there are equi-quasi-latices, $\Gamma_{i} \subset M_{i}$ and $\Gamma_{i}^{\prime} \subset M_{i}^{\prime}$, and there are points, $x_{i} \in M_{i}$ and $x_{i}^{\prime} \in M_{i}^{\prime}$, such that $r \mapsto v_{\Gamma_{i}}\left(x_{i}, r\right)$ and $r \mapsto v_{\Gamma_{i}^{\prime}}\left(x_{i}^{\prime}, r\right)$ have equi-equivalent growth.

REMARK 4.4. (i) According to Section 4.1 and Definition 4.2, the following notions make sense for the growth type of $M$ : exactly polynomial of degree $d \in \mathbb{N}$, polynomial, exponential, pseudo-quasi-polynomial, subexponential or quasi-polynomial, quasi-exponential and non-exponential.

(ii) For any quasi-lattice $\Gamma$ of $M$ and $x \in M$, the quantities

$$
\limsup _{r \rightarrow \infty} \frac{\log v_{\Gamma}(x, r)}{\log r}, \quad \liminf _{r \rightarrow \infty} \frac{\log v_{\Gamma}(x, r)}{\log r}
$$

depend only on the growth type of $M$ by 4.2 and 4.3 .

\footnotetext{
${ }^{2}$ This property is sometimes called subexponential.
} 
(iii) With the notation of Proposition 4.1 .

$$
\begin{gathered}
\liminf _{r \rightarrow \infty} \frac{\log v_{\Gamma_{1}}\left(x_{1}, r\right)}{r} \leq b \liminf _{r \rightarrow \infty} \frac{\log v_{\Gamma_{2}}\left(x_{2}, r\right)}{r}, \\
\limsup _{r \rightarrow \infty} \frac{\log v_{\Gamma_{1}}\left(x_{1}, r\right)}{r} \leq b \limsup _{r \rightarrow \infty} \frac{\log v_{\Gamma_{2}}\left(x_{2}, r\right)}{r},
\end{gathered}
$$

for any $b>Q_{R_{2}}^{1}$ by 4.1, 4.4 and 4.5.

EXAMPLE 4.5. If $M$ is the metric space of vertices of a connected graph $G$ of finite type, then $M$ is a quasi-lattice in itself, and therefore its growth type as metric space of coarse bounded geometry equals its growth type as metric space of vertices of a connected graph.

EXAMPLE 4.6. Let $M$ be a connected complete Riemannian manifold. Recall that the growth type of $M$, as Riemannian manifold, is the growth type of $r \mapsto$ $\operatorname{vol} B(x, r)$ for any $x \in M$. If $M$ is of bounded geometry, then its growth type as metric space of coarse bounded geometry equals its growth type as Riemannian manifold.

Proposition 4.7. Let $\Gamma$ be a quasi-lattice of $M, \phi: M \rightarrow M^{\prime} a(\lambda, b, c)$-large scale Lipschitz equivalence, and $x \in \Gamma$. Let $\Gamma^{\prime}=\phi(\Gamma)$ and $x^{\prime}=\phi(x)$. Then, for all $r \geq 1$,

$$
v_{\Gamma^{\prime}}\left(x^{\prime}, r\right) \leq v_{\Gamma}(x, \lambda r+b+2 c) .
$$

Proof. The result follows since

$$
B_{\Gamma^{\prime}}\left(x^{\prime}, r\right) \subset \phi\left(B_{\Gamma}(x, \lambda r+b+2 c)\right)
$$

for all $r \geq 1$ because $\phi$ is $(\lambda, b+2 c)$-large scale bi-Lipschitz by Lemma 2.21 .

Remark 4.8. In Proposition 4.7, $\Gamma^{\prime}$ is a quasi-lattice of $M^{\prime}$ by Corollary 3.11 .

Corollary 4.9. Let $\Gamma$ and $\Gamma^{\prime}$ be $\left(R, Q_{r}\right)$-quasi-lattices of $M$ and $M^{\prime}$, respectively, and let $\phi: M \rightarrow M^{\prime}$ be a $(\lambda, b, c)$-large scale Lipschitz equivalence. Take $x \in \Gamma, x^{\prime} \in \Gamma^{\prime}$ and $\delta \geq d^{\prime}\left(\phi(x), x^{\prime}\right)$. Then

$$
v_{\Gamma^{\prime}}\left(x^{\prime}, r\right) \leq p v_{\Gamma}(x, \lambda r+q)
$$

for all $r \geq 1$, where

$$
p=Q_{\lambda R+b+c}, \quad q=\lambda(\delta+\lambda R+b+c)+b+2 c .
$$

Proof. This is a direct consequence of Corollary 3.11 and Propositions 4.7 and 4.1 .

Corollary 4.10. Let $\Gamma$ and $\Gamma^{\prime}$ be $\left(R, Q_{r}\right)$-quasi-lattices of $M$ and $M^{\prime}$, respectively, and let $f: A \rightarrow A^{\prime}$ be a $(K, C)$-coarse quasi-isometry of $M$ to $M^{\prime}$. Take $x \in \Gamma, x^{\prime} \in \Gamma^{\prime}, y \in A, \delta \geq d(x, y)$ and $\delta^{\prime} \geq d^{\prime}\left(x^{\prime}, f(y)\right)$. Then

$$
v_{\Gamma^{\prime}}(x, r) \leq p v_{\Gamma}(x, C r+q)
$$

for all $r \geq 1$, where

$$
p=Q_{C R+2 C K+K}, \quad q=C\left(C \delta+4 C K+2 K+\delta^{\prime}+C R\right)+2 C K+2 K .
$$


Proof. By Proposition 2.26, $f$ is induced by a $(C, 2 C K, K)$-large scale Lipschitz equivalence $\phi: M \rightarrow M^{\prime}$. Moreover

$$
\begin{aligned}
d^{\prime}\left(\phi(x), x^{\prime}\right) \leq d^{\prime}(\phi(x), \phi(y))+d^{\prime}(\phi(y) & \left., x^{\prime}\right) \\
& \leq C d(x, y)+2 C K+\delta^{\prime} \leq C \delta+2 C K+\delta^{\prime} .
\end{aligned}
$$

Then the result follows from Corollary 4.9

Remark 4.11. According to Corollary 4.10, (equi-) coarsely quasi-isometric metric spaces of (equi-) coarse bounded geometry have (equi-) equivalent growth.

REMARK 4.12. The following version of Corollary 4.10 can be proved without using large scale Lipschitz equivalences, but using instead coarse composites (Proposition 2.12): With the hypothesis of Corollary 4.10 if $d(x, y) \leq 2 K^{*}$ and $d^{\prime}\left(x^{\prime}, f(y) \leq 2 K^{*}(5 C+1)\right.$, where $K^{*}=\max \{R, K\}$, then

$$
v_{\Gamma}\left(x_{1}, r\right) \leq Q_{\widehat{K}} \cdot v_{\Gamma^{\prime}}\left(x_{2}^{\prime}, 250 C^{4} r\right)
$$

for all $r \geq \widehat{K}$, where

$$
\widehat{K}=25 K^{\star}(5 C+1) C^{2}+5 K^{\star} C .
$$

\subsection{Growth symmetry}

Definition 4.13. A metric space, $M$, is called growth symmetric if there is a quasi-lattice $\Gamma$ in $M$ so that the growth functions $r \mapsto v_{\Gamma}(x, r)$, for all $x \in \Gamma$, equi-dominate each other.

Remark 4.14. (i) Definition 4.13 is independent of the choice of $\Gamma$ by Proposition 4.1

(ii) From Proposition 4.1, it also follows that, given quasi-lattices $\Gamma_{1}$ and $\Gamma_{2}$ of $M$, if all growth functions $r \mapsto v_{\Gamma_{1}}(x, r)$, with $x \in \Gamma_{1}$, equi-dominate all growth functions $r \mapsto v_{\Gamma_{2}}(y, r)$, with $y \in \Gamma_{2}$, then $M$ is growth symmetric.

Definition 4.15. A class $\left\{M_{i}\right\}$ is called equi-growth symmetric if there are equi-quasi-lattices $\Gamma_{i} \subset M_{i}$ so that the growth functions $r \mapsto v_{\Gamma_{i}}(x, r)$, for all $i$ and $x \in \Gamma_{i}$, equi-dominate one another.

Proposition 4.16. (Equi-)large scale Lipschitz equivalences preserve (equi)growth symmetry.

Proof. Let $\Gamma$ and $\Gamma^{\prime}$ be $\left(R, Q_{r}\right)$-quasi-lattices of $M$ and $M^{\prime}$, respectively. Assume that there are $a, b, c \geq 1$ such that $v_{\Gamma}(x, r) \leq a v_{\Gamma}(y, b r)$ for all $r \geq c$ and $x, y \in \Gamma$. Let $\phi: M \rightarrow M^{\prime}$ and $\psi: M^{\prime} \rightarrow M$ be $(\lambda, b, c)$-large scale Lipschitz equivalences so that $\psi \phi$ and $\phi \psi$ are $c$-close to $\operatorname{id}_{M}$ and $\operatorname{id}_{M^{\prime}}$, respectively. For all $x^{\prime}, y^{\prime} \in \Gamma^{\prime}$, there are $x, y \in \Gamma$ such that $d^{\prime}\left(\phi(x), x^{\prime}\right) \leq R$ and $d\left(y, \psi\left(y^{\prime}\right)\right) \leq R$. Let $p$ and $q$ be the constants defined in Corollary 4.9 with $\delta=R$. Applying Corollary 4.9 to $\phi$ and $\psi$, it follows that

$$
v_{\Gamma^{\prime}}\left(x^{\prime}, r\right) \leq p v_{\Gamma}(x, \lambda r+q) \leq a p v_{\Gamma}(y, b(\lambda r+q)) \leq a p^{2} v_{\Gamma^{\prime}}\left(y^{\prime}, \lambda b(\lambda r+q)+q\right)
$$

if $r \geq \max \left\{1, \frac{c-q}{\lambda}\right\}$.

COROLlaRY 4.17. (Equi-)coarse quasi-isometries preserve (equi-)growth symmetry.

Proof. This follows from Propositions 4.16 and 2.26 . 


\section{CHAPTER 5}

\section{Amenability of metric spaces}

In this chapter, we recall or introduce the concepts and properties about amenability on metric spaces. They needed to show some of our main result Theorems 1.10, where equi-amenability and joint amenability are relevant conditions.

\subsection{Amenability}

Suppose that $M$ and $M^{\prime}$ are of coarse bounded geometry, and $\left\{M_{i}\right\}$ and $\left\{M_{i}^{\prime}\right\}$ are of equi-coarse bounded geometry.

Definition 5.1 (Block-Weinberger [20]). A metric space, $M$, is called amenable if it has a quasi-lattice $\Gamma$ and a sequence of finite subsets $S_{n} \subset \Gamma$ such that $\left|\partial_{r}^{\Gamma} S_{n}\right| /\left|S_{n}\right| \rightarrow 0$ as $n \rightarrow \infty$ for each $r>0$. Such a sequence $S_{n}$ is called a Følner sequence in $\Gamma$.

EXAMPLE 5.2. If $M$ is the metric space of vertices of some connected graph of finite type, then $M$ is amenable if and only it is Følner as metric space of vertices of some connected graph (cf. (3.7)).

EXAMPLE 5.3. Let $M$ is a connected complete Riemannian manifold. Recall that a Riemannian manifold is called $F \varnothing l n e r$ if it has a sequence of smooth compact domains $\Omega_{n}$ such that $\operatorname{vol} \partial \Omega_{n} / \operatorname{vol} \Omega_{n} \rightarrow 0$ as $n \rightarrow \infty$. If $M$ is of bounded geometry, then it is amenable as metric space of coarse bounded geometry if and only if it is Følner as Riemannian manifold.

Definition 5.4. $\left\{M_{i}\right\}$ is called weakly equi-amenable when

- there are equi-quasi-lattices $\Gamma_{i} \subset M_{i}$;

- there are subsets $S_{i, m, n} \subset \Gamma_{i}(m, n \in \mathbb{N})$;

- there is $a \geq 1$, and mappings $r \mapsto p_{r}\left(r, p_{r}>0\right)$ and $t \mapsto q_{t}\left(t, q_{t} \geq 0\right)$; and

- there is a nonempty subset $\mathcal{N}_{i, j, m, n, t} \subset \mathbb{N}$ for every $i, j, m, n$ and $t$; such that:

- for each $i$ and $m$, the sequence $S_{i, m, n}$ is Følner in $\Gamma_{i}$; and,

- for each $i, m$ and $t$, and any Følner sequence $X_{n}$ of $\Gamma_{i}$ with $S_{i, m, n} \subset X_{n} \subset$ $\operatorname{Pen}_{\Gamma_{i}}\left(S_{i, m, n}, t\right)$, there is some $Y_{i, j, m^{\prime}, n} \subset \Gamma_{j}$ for all $j, n$ and $m^{\prime} \in \mathcal{N}_{i, j, m, n, t}$ so that

$$
\begin{gathered}
S_{j, m^{\prime}, n} \subset Y_{i, j, m^{\prime}, n} \subset \operatorname{Pen}_{\Gamma_{i}}\left(S_{j, m^{\prime}, n}, q_{t}\right), \\
\frac{\left|\partial_{r}^{\Gamma_{j}} Y_{i, j, m^{\prime}, n}\right|}{\left|Y_{i, j, m^{\prime}, n}\right|} \leq a \frac{\left|\partial_{p_{r}}^{\Gamma_{i}} X_{n}\right|}{\left|X_{n}\right|},
\end{gathered}
$$

for all $r$.

If moreover the mappings $r \mapsto p_{r}$ and $t \mapsto q_{t}$ can be chosen to be affine, then $\left\{M_{i}\right\}$ is called equi-amenable. 
Proposition 5.5. The following are true:

( $i$ ) (Equi-)rough equivalences preserve (weak equi-) amenability.

(ii) (Equi-) coarse quasi-isometries preserve (equi-)amenability.

Proof. Let $f: M \rightarrow M^{\prime}$ be an $\left(s_{r}, c\right)$-rough equivalence. By Definition 2.35. there is an $s_{r}$-rough map $g: M^{\prime} \rightarrow M$ such that $g f$ and $f g$ are $c$-close to id $M$ and $\operatorname{id}_{M^{\prime}}$, respectively. Let $\Gamma$ be an $\left(R, Q_{r}\right)$-quasi-lattice of $M$, for some coarse bound $\left(R, Q_{r}\right)$. By Proposition 3.10, $\Gamma^{\prime}=f(\Gamma)$ is an $\left(R^{\prime}, Q_{r}^{\prime}\right)$-quasi-lattice of $M^{\prime}$, where $R^{\prime}=c+s_{R}$ and $Q_{r}^{\prime}=Q_{s_{r+R^{\prime}}}$.

Let $S \subset \Gamma$ be finite and let $S^{\prime}=f(S) \subset \Gamma^{\prime}$. Then $S \subset \operatorname{Pen}_{\Gamma}\left(g\left(S^{\prime}\right), c\right)$, and so

$$
|S| \leq Q_{c}\left|S^{\prime}\right|
$$

Claim 1. $\partial_{r}^{\Gamma^{\prime}} S^{\prime} \subset f\left(\partial_{s_{r}}^{\Gamma} S\right)$ for all $r>0$.

Indeed, let $x^{\prime} \in \partial_{r}^{\Gamma^{\prime}} S^{\prime}$ and $x \in \Gamma$ with $f(x)=x^{\prime}$. There are points $y^{\prime} \in S^{\prime}$ and $z^{\prime} \in \Gamma^{\prime} \backslash S^{\prime}$ such that $d^{\prime}\left(x^{\prime}, y^{\prime}\right) \leq r$ and $d^{\prime}\left(x^{\prime}, z^{\prime}\right) \leq r$. If $y \in S$ and $z \in \Gamma \backslash S$ are such that $f(y)=y^{\prime}$ and $f(z)=z^{\prime}$, then $d(x, y) \leq s_{r}$ and $d(x, z) \leq s_{r}$, and so $x \in \partial_{s_{r}}^{\Gamma} S$.

It follows from Claim 1 that

$$
\left|\partial_{r}^{\Gamma^{\prime}} S^{\prime}\right| \leq\left|\partial_{s_{r}}^{\Gamma} S\right|
$$

and combining 5.3 and 5.4 that

$$
\frac{\left|\partial_{r}^{\Gamma^{\prime}} S^{\prime}\right|}{\left|S^{\prime}\right|} \leq Q_{c} \frac{\left|\partial_{s_{r}}^{\Gamma} S\right|}{|S|}
$$

If $S_{n}$ is a Følner sequence in $\Gamma$, then $S_{n}^{\prime}$ is a Følner sequence in $\Gamma^{\prime}$ by $(5.5)$, and therefore $M^{\prime}$ is amenable. This shows the weaker version of (i). Then the weaker version of (ii) follows by Propositions 2.26 and 2.39. (2).

Claim 2. $f\left(\partial_{r}^{\Gamma} \operatorname{Pen}_{\Gamma}(S, 2 c)\right) \subset \partial_{u_{r}}^{\Gamma^{\prime}} S^{\prime}$ for all $r>0$, where $u_{r}=s_{r}+s_{2 c}$

Let $x \in \partial_{r}^{\Gamma} \operatorname{Pen}_{\Gamma}(S, 2 c)$. There are some $y \in \operatorname{Pen}_{\Gamma}(S, 2 c)$ and $z \in \Gamma \backslash \operatorname{Pen}_{\Gamma}(S, 2 c)$ such that $d(x, y) \leq r$ and $d(x, z) \leq r$. Then there is some $y_{0} \in S$ so that $d\left(y, y_{0}\right) \leq 2 c$. Let $x^{\prime}=f(x), y^{\prime}=f(y)$ and $z^{\prime}=f(z)$ in $\Gamma^{\prime}$, and $y_{0}^{\prime}=f\left(y_{0}\right)$ in $S^{\prime}$. We have $z^{\prime} \in \Gamma^{\prime} \backslash S^{\prime}$; otherwise, $z^{\prime}=f\left(z_{0}\right)$ for some $z_{0} \in S$, obtaining

$$
d\left(z, z_{0}\right) \leq d\left(z, g\left(z^{\prime}\right)\right)+d\left(g\left(z^{\prime}\right), z_{0}\right) \leq 2 c,
$$

which is a contradiction because $z \notin \operatorname{Pen}_{\Gamma}(S, 2 c)$. Moreover

$$
d^{\prime}\left(x^{\prime}, y_{0}^{\prime}\right) \leq d^{\prime}\left(x^{\prime}, y^{\prime}\right)+d^{\prime}\left(y^{\prime}, y_{0}^{\prime}\right) \leq s_{r}+s_{2 c}=u_{r}
$$

and $d^{\prime}\left(x^{\prime}, z^{\prime}\right) \leq s_{r}$, giving $x^{\prime} \in \partial_{u_{r}}^{\Gamma^{\prime}} S^{\prime}$, which shows Claim 2 ,

Applying (5.3) to the set $\partial_{r}^{\Gamma} \operatorname{Pen}_{\Gamma}(S, 2 c)$, and using Claim 2, we get

$$
\frac{\left|\partial_{r}^{\Gamma} \operatorname{Pen}_{\Gamma}(S, 2 c)\right|}{\left|\operatorname{Pen}_{\Gamma}(S, 2 c)\right|} \leq Q_{c} \frac{\left|f\left(\partial_{r}^{\Gamma} \operatorname{Pen}_{\Gamma}(S, 2 c)\right)\right|}{|S|} \leq Q_{c} \frac{\left|\partial_{u_{r}}^{\Gamma^{\prime}} S^{\prime}\right|}{\left|S^{\prime}\right|} .
$$

Suppose that $\left\{M_{i}\right\}$ satisfies the condition of weak equi-amenability (Definition 5.4 with a class $\left\{\Gamma_{i}\right\}$ of corresponding $\left(R, Q_{r}\right)$-quasi-lattices, subsets $S_{i, m, n} \subset$ $\Gamma_{i}$, a constant $a \geq 1$, mappings $r \mapsto p_{r}$ and $t \mapsto q_{t}$, and nonempty subsets $\mathcal{N}_{i, j, m, n, t} \subset$ $\mathbb{N}$. Consider a family of equi-rough equivalences $f_{i}: M_{i} \rightarrow M_{i}^{\prime}$ with common rough equivalence distortion $\left(s_{r}, c\right)$. By Proposition 3.10 each $\Gamma_{i}^{\prime}=f_{i}\left(\Gamma_{i}\right)$ is an $\left(R^{\prime}, Q_{r}^{\prime}\right)$ quasi-lattice of $M_{i}^{\prime}$, where $R^{\prime}=c+s_{R}$ and $Q_{r}^{\prime}=Q_{s_{r+R^{\prime}}}$. By [5.5), for each $i$ and 
$m, S_{i, m, n}^{\prime}=f_{i}\left(S_{i, m, n}\right)$ is a Følner sequence in $\Gamma_{i}^{\prime}$. Given $i, m$ and $t^{\prime} \geq 0$, take any Følner sequence $X_{n}^{\prime}$ in any $\Gamma_{i}^{\prime}$ with $S_{i, m, n}^{\prime} \subset X_{n}^{\prime} \subset \operatorname{Pen}_{\Gamma_{i}^{\prime}}\left(S_{i, m, n}^{\prime}, t^{\prime}\right)$. Then

$$
X_{n}:=\operatorname{Pen}_{\Gamma_{i}}\left(f_{i}^{-1}\left(X_{n}^{\prime}\right), 2 c\right) \subset \operatorname{Pen}_{\Gamma_{i}}\left(S_{i, m, n}, s_{t^{\prime}}+2 c\right) ;
$$

in particular, every $X_{n}$ is finite. Moreover $X_{n}$ is a Følner sequence in $\Gamma_{i}$ by (5.6) since $f_{i}\left(f_{i}^{-1}\left(X_{n}^{\prime}\right)\right)=X_{n}^{\prime}$. Thus (5.1) and (5.2) are satisfied with $t=s_{t^{\prime}}+2 c$ and some subsets $Y_{j, m^{\prime}, n} \subset \Gamma_{j}\left(m^{\prime} \in \mathcal{N}_{i, j, m, n}\right)$. Let $q_{t^{\prime}}^{\prime}=s_{q_{t}}$ and $Y_{j, m^{\prime}, n}^{\prime}=f_{j}\left(Y_{j, m^{\prime}, n}\right) \subset \Gamma_{j}^{\prime}$ for $m^{\prime} \in \mathcal{N}_{i, j, m, n}$. We have

$$
S_{j, m^{\prime}, n}^{\prime} \subset Y_{j, m^{\prime}, n}^{\prime} \subset \operatorname{Pen}_{\Gamma_{j}^{\prime}}\left(S_{j, m^{\prime}, n}^{\prime}, q_{t^{\prime}}^{\prime}\right) .
$$

For $r^{\prime}>0$, let $r=s_{r^{\prime}}$ and $p_{r^{\prime}}^{\prime}=u_{p_{r}}$. By (5.5), (5.2) and (5.6),

$$
\frac{\left|\partial_{r^{\prime}}^{\Gamma_{j}^{\prime}} Y_{j, m^{\prime}, n}^{\prime}\right|}{\left|Y_{j, m^{\prime}, n}^{\prime}\right|} \leq Q_{c} \frac{\left|\partial_{r}^{\Gamma_{j}} Y_{j, m^{\prime}, n}\right|}{\left|Y_{j, m^{\prime}, n}\right|} \leq a Q_{c} \frac{\left|\partial_{p_{r}}^{\Gamma_{i}} X_{n}\right|}{\left|X_{n}\right|} \leq a Q_{c}^{2} \frac{\left|\partial_{p_{r^{\prime}}^{\prime}}^{\Gamma_{i}} X_{n}^{\prime}\right|}{\left|X_{n}^{\prime}\right|} .
$$

for all $r^{\prime}$. So $\left\{M_{i}^{\prime}\right\}$ is weakly equi-amenable (the stronger version of (ii)).

The stronger version of (ii) follows like the stronger version of (ii), assuming that the family $\left\{f_{i}\right\}$ is equi-large scale Lipschitz by Propositions 2.26 and 2.27. and using the expression of $s_{r}$ in Proposition 2.39 2 and the expression of $u_{r}$ in Claim 2,

Remark 5.6. In the proof of Proposition 5.5, since the fibers of $f$ are of diameter $\leq s_{0}$, we could use $Q_{s_{0}}$ instead of $Q_{c}$ in (5.3), and Claim 2 could be stated with $s_{0}$ instead of $2 c$.

REMARK 5.7. The weaker version of Proposition 5.5.(i) was proved by Block and Weinberger [20. Their proof has the following three steps. First, they introduce the uniformly finite homology, $H_{\bullet}^{\text {uf }}(M)$; second, they show that $H_{\bullet}^{\text {uf }}(M) \cong$ $H_{\bullet}^{\mathrm{uf}}\left(M^{\prime}\right)$ if $M$ and $M^{\prime}$ are roughly equivalent; and third, they prove that $M$ is amenable if and only if $H_{0}^{\mathrm{uf}}(M) \neq 0$.

Proposition 5.8. If $M$ is amenable, then each quasi-lattice of $M$ has a Følner sequence.

Proof. Let $\Gamma$ be a quasi-lattice of $M$. Since $M$ is amenable, $\Gamma$ is also amenable by Proposition 5.5. Therefore some $\left(R, Q_{r}\right)$-quasi-lattice $\Gamma_{0}$ of $\Gamma$ has a Følner sequence $S_{0, n}$. It has to be shown that $\Gamma$ also has a Følner sequence. Let $S_{n}=$ $\operatorname{Pen}_{\Gamma}\left(S_{0, n}, R\right)$.

Claim 3. $\partial_{r}^{\Gamma} S_{n} \subset \operatorname{Pen}_{\Gamma}\left(\partial_{r+2 R}^{\Gamma_{0}} S_{0, n}, R\right)$ for all $r>0$.

For each $x \in \partial_{r}^{\Gamma} S_{n}$, there are $x_{0} \in \Gamma_{0}, y \in S_{n}$ and $z \in \Gamma \backslash S_{n}$ such that $d\left(x, x_{0}\right) \leq R$, $d(x, y) \leq r$ and $d(x, z) \leq r$. Then there are points $y_{0} \in S_{0, n}$ and $z_{0} \in \Gamma_{0}$ such that $d\left(y, y_{0}\right) \leq R$ and $d\left(z, z_{0}\right) \leq R$. Observe that $z_{0} \notin S_{0, n}$ because $z \notin S_{n}$ and $d\left(z, z_{0}\right) \leq R$. By the triangle inequality, the distances $d\left(x_{0}, y_{0}\right) \leq r+2 R$ and $d\left(x_{0}, z_{0}\right) \leq r+2 R$, and so $x_{0} \in \partial_{r+2 R}^{\Gamma_{0}} S_{0, n}$. Hence $x \in \operatorname{Pen}_{\Gamma}\left(\partial_{r+2 R}^{\Gamma_{0}} S_{0, n}, R\right)$, which completes the proof of Claim 3 .

By Claim 3 ,

$$
\frac{\left|\partial_{r}^{\Gamma} S_{n}\right|}{\left|S_{n}\right|} \leq \frac{\left|\operatorname{Pen}_{\Gamma}\left(\partial_{r+2 R}^{\Gamma_{0}} S_{0, n}, R\right)\right|}{\left|S_{0, n}\right|} \leq Q_{R} \frac{\left|\partial_{r+2 R}^{\Gamma_{0}} S_{0, n}\right|}{\left|S_{0, n}\right|} .
$$

Then $S_{n}$ is a Følner sequence in $\Gamma$ because $S_{0, n}$ is a Følner sequence in $\Gamma_{0}$. 
REMARK 5.9. In the proof of Proposition 5.8, it is easy to check that $\partial_{r}^{\Gamma_{0}}\left(S_{n} \cap\right.$ $\left.\Gamma_{0}\right) \subset \partial_{r}^{\Gamma} S_{n}$ for all $r>0$, and therefore

$$
\frac{\left|\partial_{r}^{\Gamma_{0}}\left(S_{n} \cap \Gamma_{0}\right)\right|}{\left|S_{n} \cap \Gamma_{0}\right|} \leq \frac{\left|\partial_{r}^{\Gamma} S_{n}\right|}{\left|S_{n}\right|} .
$$

\subsection{Amenable symmetry}

Definition 5.10. A metric space, $M$, is called weakly amenably symmetric if there are:

- a quasi-lattice $\Gamma$ of $M$;

- subsets $S_{m, n} \subset \Gamma(m, n \in \mathbb{N})$;

- some $a \geq 1$, and mappings $r \mapsto p_{r}\left(r, p_{r}>0\right)$ and $t \mapsto q_{t}\left(t, q_{t} \geq 0\right)$; and

- a nonempty subset $\mathcal{N}_{m, n, t} \subset \mathbb{N}$ for each $m, n$ and $t$;

such that:

- for each $m$, the sequence $S_{m, n}$ is Følner in $\Gamma_{i}$;

- for each $m, n$ and $t \geq 0, \cup_{m^{\prime} \in \mathcal{N}_{m, n, t}} S_{m^{\prime}, n}$ is a net in $M$; and

- for each $m$ and $t \geq 0$, and any Følner sequence $X_{n}$ of $\Gamma$ with $S_{m, n} \subset X_{n} \subset$ $\operatorname{Pen}_{\Gamma}\left(S_{m, n}, t\right)$, there is some $Y_{m^{\prime}, n} \subset \Gamma$ for each $n$ and $m^{\prime} \in \mathcal{N}_{m, n}$ so that

$$
\begin{gathered}
S_{m^{\prime}, n} \subset Y_{m^{\prime}, n} \subset \operatorname{Pen}_{\Gamma_{i}}\left(S_{m^{\prime}, n}, q_{t}\right), \\
\frac{\left|\partial_{r}^{\Gamma} Y_{m^{\prime}, n}\right|}{\left|Y_{m^{\prime}, n}\right|} \leq a \frac{\left|\partial_{p_{r}}^{\Gamma} X_{n}\right|}{\left|X_{n}\right|},
\end{gathered}
$$

for all $r$.

In this case, $\Gamma$ is called weakly Følner symmetric. If moreover the mappings $r \mapsto p_{r}$ and $t \mapsto q_{t}$ can be chosen to be affine, then $M$ is called amenably symmetric and $\Gamma$ is called Følner symmetric.

ExAmPLE 5.11. Let $M \subseteq \mathbb{R}^{3}$ be the union of a plane and a line orthogonal to the plane. With the subspace metric, $M$ is amenable but not amenably symmetric.

Definition 5.12. A class of metric spaces, $\left\{M_{i}\right\}$, is (weakly) equi-amenably symmetric if every $M_{i}$ satisfies the conditions of (weak) amenable symmetry (Definition 5.10 with equi-quasi-lattices $\Gamma_{i} \subset M_{i}$, subsets $S_{i, m, n} \subset \Gamma_{i}$, the same constant $a \geq 1$, the same mappings $r \mapsto p_{r}$ and $t \mapsto q_{t}$, and subsets $\mathcal{N}_{i, m, n, t} \subset \mathbb{N}$, such that, for some $L_{n}, L_{i, m, n, t} \in \mathbb{N}, \bigcup_{m} S_{i, m, n}$ is an $L_{n}$-net of $\Gamma_{i}$ for all $i$, and $\bigcup_{m^{\prime} \in \mathcal{N}_{i, j, m, n, t}} S_{j, m^{\prime}, n}$ is an $L_{i, m, n, t}$-net of $\Gamma_{j}$ for all $j$.

REMARK 5.13. In Definition 5.12 every $M_{i}$ satisfies the conditions of (weak) amenable symmetry with subsets $\mathcal{N}_{i, m, n, t} \subset \mathbb{N}$ that may depend on $i$.

Proposition 5.14. (i) (Equi-)rough equivalences preserve weak (equi-)amenable symmetry.

(ii) (Equi-) coarse quasi-isometries preserve (equi-)amenable symmetry.

Proof. Let $f: M \rightarrow M^{\prime}$ be a rough equivalence with rough equivalence distortion $\left(s_{r}, c\right)$. Suppose that $M$ satisfies the conditions to be weakly amenably symmetric with a quasi-lattice $\Gamma$, sets $S_{m, n}$, a constant $a \geq 1$, mappings $r \mapsto p_{r}$ and $t \mapsto q_{t}$, and subsets $\mathcal{N}_{m, n, t} \subset \mathbb{N}$; in particular, each union $\bigcup_{m^{\prime} \in \mathcal{N}_{m, n, t}} S_{m^{\prime}, n}$ is a $K_{m, n, t}$-net in $M$ for some $K_{m, n, t} \geq 0$. Let $S_{m, n}^{\prime}=f\left(S_{m, n}\right)$. For each $m$, the sequence $S_{m, n}^{\prime}$ is Følner in $\Gamma^{\prime}$ by 5.5 . Moreover $\cup_{m^{\prime} \in \mathcal{N}_{m, n, t}} S_{m^{\prime}, n}^{\prime}$ is a $\left(s_{K_{m, n, t}}+c\right)$-net in $M^{\prime}$, 
which can be proved as follows. For each $x^{\prime} \in M^{\prime}$, there is some $y \in \cup_{m^{\prime} \in \mathcal{N}_{m, n, t}} S_{m^{\prime}, n}$ such that $d\left(y, g\left(x^{\prime}\right)\right) \leq K_{n}$. Then $f(y) \in \cup_{m^{\prime} \in \mathcal{N}_{m, n, t}} S_{m^{\prime}, n}^{\prime}$ and

$$
d^{\prime}\left(f(y), x^{\prime}\right) \leq d^{\prime}\left(f(y), f g\left(x^{\prime}\right)\right)+d^{\prime}\left(f g\left(x^{\prime}\right), x^{\prime}\right) \leq s_{K_{m, n, t}}+c .
$$

The rest of the proof is analogous to the proof of Proposition 5.5

Proposition 5.15. If $M$ is (weakly) amenably symmetric, then every quasilattice of $M$ is (weakly) Følner symmetric.

Proof. Suppose that $M$ is (weakly) amenably symmetric, and let $\Gamma$ be a quasilattice of $M$. By Proposition 5.14. $\Gamma$ satisfies the condition of (weak) amenable symmetry (Definition 5.10 with some $\left(R, Q_{r}\right)$-quasi-lattice $\Gamma^{\prime}$ of $\Gamma$, a family of Følner sequences $S_{m, n}^{\prime}$ in $\Gamma^{\prime}$, some constant $a^{\prime} \geq 1$, and some mappings $r \mapsto p_{r}^{\prime}$ and $t \mapsto q_{t}^{\prime}$. For each $m$ and $n$, let $S_{m, n}=\operatorname{Pen}_{\Gamma}\left(S_{m, n}^{\prime}, R\right)$. For every $m, S_{m, n}$ is a Følner sequence in $\Gamma$ by (5.7). Given $m$ and $t \geq 0$, let $X_{n}$ be a Følner sequence in $\Gamma$ with $S_{m, n} \subset X_{n} \subset \operatorname{Pen}_{\Gamma}\left(S_{m, n}, t\right)$. Then $X_{n}^{\prime}:=X_{n} \cap \Gamma^{\prime}$ is a Følner sequence in $\Gamma^{\prime}$ by Remark 5.9 and moreover $S_{m, n}^{\prime} \subset X_{n}^{\prime} \subset \operatorname{Pen}_{\Gamma^{\prime}}\left(S_{m, n}^{\prime}, t+R\right)$. Hence $X_{n}^{\prime}$ satisfies (5.8) and (5.9) with some subsets $Y_{m, n}^{\prime} \subset \Gamma^{\prime}$, using $q_{t+R}^{\prime}$. For $Y_{m, n}=\operatorname{Pen}_{\Gamma}\left(Y_{m, n}^{\prime}, R\right)$, we have

$$
\begin{gathered}
S_{m, n} \subset Y_{m, n} \subset \operatorname{Pen}_{\Gamma}\left(\operatorname{Pen}_{\Gamma^{\prime}}\left(S_{m, n}^{\prime}, q_{t+R}^{\prime}\right), R\right) \subset \operatorname{Pen}_{\Gamma}\left(S_{m, n}, q_{t+R}^{\prime}+R\right), \\
\frac{\left|\partial_{r}^{\Gamma} Y_{m, n}\right|}{\left|Y_{m, n}\right|} \leq Q_{R} \frac{\left|\partial_{r}^{\Gamma^{\prime}} Y_{m, n}^{\prime}\right|}{\left|Y_{m, n}^{\prime}\right|} \leq a^{\prime} Q_{R} \frac{\left|\partial_{p_{r}^{\prime}}^{\Gamma^{\prime}} X_{n}^{\prime}\right|}{\left|X_{n}^{\prime}\right|} \leq a^{\prime} Q_{R} \frac{\left|\partial_{p_{r}^{\prime}}^{\Gamma} X_{n}\right|}{\left|X_{n}\right|},
\end{gathered}
$$

by 2.2 and (5.7)-(5.9). Thus the condition of (weak) amenable symmetry of $M$ is satisfied with $\Gamma$.

Definition 5.16. A class of metric spaces, $\left\{M_{i}\right\}$, is jointly weakly amenably symmetric if there are:

- equi-quasi-lattices $\Gamma_{i} \subset M_{i}$;

- subsets $S_{i, m, n} \subset \Gamma_{i}(m, n \in \mathbb{N})$;

- mappings $r \mapsto p_{r}\left(r, p_{r}>0\right)$ and $t \mapsto q_{t}\left(t, q_{t} \geq 0\right)$;

- nonempty subsets $\mathcal{N}_{i, j, m, n, t} \subset \mathbb{N}$, one for each $i, j, m, n$ and $t$;

- numbers $L_{n}, L_{i, m, n, t} \in \mathbb{N}$, one for each $i, m, n$ and $t$; and $a \geq 1$, such that:

- for each $i$ and $m$, the sequence $S_{i, m, n}$ is Følner in $\Gamma_{i}$;

- for each $i$ and $n, \cup_{m} S_{i, m, n}$ is an $L_{n}$-net in $M_{i}$;

- for each $i, j, m, n$ and $t, \cup_{m^{\prime} \in \mathcal{N}_{i, j, m, n, t}} S_{j, m^{\prime}, n}$ is an $L_{i, m, n, t}$-net in $M_{j}$; and

- for each $i, m$ and $t$, and any Følner sequence $X_{n}$ of $\Gamma_{i}$ with $S_{i, m, n} \subset X_{n} \subset$ $\operatorname{Pen}_{\Gamma}\left(S_{i, m, n}, t\right)$, there is some $Y_{i, j, m^{\prime}, n} \subset \Gamma_{j}$ for all $j, n$ and $m^{\prime} \in \mathcal{N}_{m, n}$ so that

$$
\begin{gathered}
S_{j, m^{\prime}, n} \subset Y_{i, j, m^{\prime}, n} \subset \operatorname{Pen}_{\Gamma_{i}}\left(S_{i, m^{\prime}, n}, q_{t}\right), \\
\frac{\left|\partial_{r}^{\Gamma} Y_{i, j, m^{\prime}, n}\right|}{\left|Y_{i, j, m^{\prime}, n}\right|} \leq a \frac{\left|\partial_{p_{r}}^{\Gamma} X_{n}\right|}{\left|X_{n}\right|},
\end{gathered}
$$

for all $r$.

In this case, $\left\{\Gamma_{i}\right\}$ is called jointly weakly Følner symmetric. If moreover the mappings $r \mapsto p_{r}$ and $t \mapsto q_{t}$ can be chosen to be affine, then $\left\{M_{i}\right\}$ is called jointly amenably symmetric and $\left\{\Gamma_{i}\right\}$ jointly Følner symmetric. 
REMARK 5.17. Joint (weak) amenable symmetry is stronger than (weak) equiamenability and (weak) equi-amenable symmetry.

Proposition 5.18. (i) Equi-rough equivalences preserve weak joint amenable symmetry.

(ii) Equi-coarse quasi-isometries preserve joint amenable symmetry.

Proof. Similar to the proof of Proposition 5.14. 


\section{CHAPTER 6}

\section{Coarse ends}

The end space is a topological invariant. In the case of proper geodesic spaces, the end space turns out to be a coarsely quasi-isometric invariant 21, Proposition 8.29]. Proceeding further with this idea, we introduce a version of the end space for metric spaces, which is invariant by coarse equivalences. Indeed it can be directly generalized to arbitrary coarse spaces.

\subsection{Ends}

The end space of a topological space $X$ is constructed as follows. Let $\mathcal{K}$ be the family of compact subsets of $X$. For each $K \in \mathcal{K}$, let $\mathcal{U}_{K}$ be the discrete space of connected components of $X \backslash K$ with non-compact closure. Each inclusion $K \subset L$ in $\mathcal{K}$ induces a map $\eta_{K, L}: \mathcal{U}_{L} \rightarrow \mathcal{U}_{K}$ which assigns to $U \in \mathcal{U}_{L}$ the component $\eta_{K, L}(U) \in \mathcal{U}_{K}$ which contains $U$. These spaces $\mathcal{U}_{K}$ and maps $\eta_{K, L}, K, L \in \mathcal{K}$, form an inverse system, whose inverse limit is the space of ends of $X$, denoted by $\mathcal{E}(X)$, which is Hausdorff and totally disconnected. Thus any $\mathbf{e} \in \mathcal{E}(X)$ can be described as a map defined on $\mathcal{K}$ such that $\mathbf{e}(K) \in \mathcal{U}_{K}$ and $\mathbf{e}(K) \supset \mathbf{e}(L)$ if $K \subset L$ in $\mathcal{K}$. Suppose that $X$ has a an increasing sequence of compact subsets, $\left(K_{n}\right)$, whose interiors cover $X$. Then the topology of $\mathcal{E}(X)$ is induced by the ultrametric $d_{\left(K_{n}\right)}$ defined by

$$
d_{\left(K_{n}\right)}(\mathbf{e}, \mathbf{f})=\exp \left(-\sup \left\{n \in \mathbb{N} \mid \mathbf{e}\left(K_{n}\right)=\mathbf{f}\left(K_{n}\right)\right\}\right) .
$$

The coarse version of these concepts for metric spaces is obtained by replacing compact sets by bounded sets, as shown next.

\subsection{Coarse connectivity}

Definition 6.1. Let $\mu>0$. Two points $x, y \in M$ are coarsely $\mu$-connected if there is, for some $k$, a finite sequence $\left\{z_{l}\right\}_{l=0}^{k}$ in $M$ such that $x=z_{0}, z_{1}, \ldots, z_{k}=y$ and that $d\left(z_{l-1}, z_{l}\right) \leq \mu$ for all $l \in\{1, \ldots, k\}$. This concept defines an equivalence relation on $M$ whose equivalence classes are called coarse $\mu$-connected components. If all points in $M$ are $\mu$-connected, then $M$ is called coarsely $\mu$-connected. If $M$ is coarsely $\mu$-connected for some $\mu>0$, then $M$ is called coarsely connected.

REMARK 6.2. The following properties are elementary:

(i) The coarse $\mu$-connected components are the maximal coarse $\mu$-connected subsets.

(ii) If $M$ is coarsely $\mu$-connected, then it is coarsely $\nu$-connected for all $\nu \geq \mu$.

(iii) If $M$ is coarsely $\mu$-connected, then, for all non-trivial partition of $M$ into two sets, $\{A, B\}$, there is some $x \in A$ and $y \in B$ such that $d(x, y) \leq \mu$.

(iv) If $A$ and $B$ are coarsely $\mu$-connected subsets of $M$, and $d(x, y) \leq \mu$ for some $x \in A$ and $y \in B$, then $A \cup B$ is coarsely $\mu$-connected. 
Remark 6.3. Coarse connectivity of $M$ means that the coarse space $[M]$ is monogenic [86, but we prefer the term coarse connectivity because it plays the same role here as connectivity in the definition of ends.

Lemma 6.4. If $M$ is coarsely $\mu$-connected, $f: M \rightarrow M^{\prime}$ satisfies the condition of uniform expansiveness with the mapping $s_{r}$, and $f(M)$ is a c-net in $M^{\prime}$, then $M^{\prime}$ is coarsely $\mu^{\prime}$-connected, where $\mu^{\prime}=\max \left\{s_{\mu}, c\right\}$.

Proof. For $x^{\prime}, y^{\prime} \in M^{\prime}$, there are points $x, y \in M$ such that $d^{\prime}\left(x^{\prime}, f(x)\right) \leq c$ and $d^{\prime}\left(y^{\prime}, f(y)\right) \leq c$, and there is a finite sequence $x=z_{0}, z_{1}, \ldots, z_{k}=y$ in $M$ such that $d\left(z_{l-1}, z_{l}\right) \leq \mu$ for all $l \in\{1, \ldots, k\}$. Then $d^{\prime}\left(f\left(z_{l-1}\right), f\left(z_{l}\right)\right) \leq s_{\mu}$, obtaining that $x^{\prime}$ is coarsely $\mu^{\prime}$-connected to $y^{\prime}$.

COROLlary 6.5. Coarse connectivity is invariant by coarse equivalences.

Remark 6.6. Corollary 6.5 is indeed trivial by Remark 6.3.

Lemma 6.7. Let $B \subset C \subset M$, and let $U$ be a coarse $\mu$-connected component of $M \backslash B$. Then each coarse $\mu$-connected component of $U \backslash C$ is a coarse $\mu$-connected component of $M \backslash C$.

Proof. Each coarse $\mu$-connected component $V$ of $U \backslash C$ is contained in some coarse $\mu$-connected component $W$ of $M \backslash C$ (Remark 6.2 (ii)). If $V \neq W$, then there are points, $y \in V$ and $z \in W \backslash V$, such that $d(y, z) \leq \mu$ (Remark 6.2 (iii)). Hence $z$ is coarsely $\mu$-connected to $y$ in $M \backslash C$, and therefore in $M \backslash B$. So $z \in U$, and $z$ is coarsely $\mu$-connected to $y$ in $U \backslash C$, giving $z \in V$, a contradiction. Thus $V=W$.

Corollary 6.8. Let $A, B \subset M$, and let $U$ be a coarse $\mu$-connected component of $M \backslash B$ such that $U \cap A=\varnothing$. Then $U$ is a coarse $\mu$-connected component of $M \backslash(A \cup B)$.

Proof. Take $C=A \cup B$ in Lemma 6.7

Lemma 6.9. Suppose that $M$ is coarsely $\mu$-connected. Let $\varnothing \neq B \mp M$, and let $U$ be a coarse $\mu$-connected component of $M \backslash B$. Then there are points $x \in B$ and $y \in U$ such that $d(x, y) \leq \mu$.

Proof. Let $x_{0} \in B$ and $y_{0} \in U \backslash B$. Since $M$ is coarsely $\mu$-connected, there is a finite sequence $x_{0}=z_{0}, z_{1}, \ldots, z_{k}=y_{0}$ such that $d\left(z_{l-1}, z_{l}\right) \leq \mu$ for all $l \in\{1, \ldots, k\}$. Let

$$
p=\min \left\{l \in\{1, \ldots, k\} \mid\left\{z_{l}, \ldots, z_{k}\right\} \subset M \backslash B\right\} .
$$

Then the statement holds with $x=z_{p-1}$ and $y=z_{p}$.

REMARK 6.10. Lemma 6.9 is a refinement of Remark 6.2 (iii).

Corollary 6.11. Suppose that $M$ is coarsely $\mu$-connected. Let $A$ be a $\mu$-net of $M$, let $\varnothing \neq B \subset M$, and let $U$ be a coarse $\mu$-connected component of $M \backslash B$ such that $U \backslash \operatorname{Pen}(B, 2 \mu) \neq \varnothing$. Then $A \cap U \cap \operatorname{Pen}(B, 3 \mu) \neq \varnothing$.

Proof. The set $U \backslash \operatorname{Pen}(B, 2 \mu)$ is a coarse $\mu$-connected component of $M$ 、 $\operatorname{Pen}(B, 2 \mu)$ by Lemma 6.7. Then, by Lemma 6.9, there are points $x \in \operatorname{Pen}(B, 2 \mu)$ and $y \in U \backslash \operatorname{Pen}(B, 2 \mu)$ with $d(x, y) \leq \mu$. Since $x \in M \backslash \operatorname{Pen}(B, \mu)$ by the triangle inequality, we also get $x \in U$. Take some $z \in A$ such that $d(x, z) \leq \mu$. Since $z \in \operatorname{Pen}(B, 3 \mu) \backslash B$ by the triangle inequality, we have $z \in A \cap U \cap \operatorname{Pen}(B, 3 \mu)$. 
Corollary 6.12. Suppose that $M$ is of coarse bounded geometry with coarse bound $\left(R, Q_{r}\right)$, and coarsely $\mu$-connected for some $\mu \geq R$. Let $B$ be a nonempty bounded subset of $M$. Then $M \backslash B$ has at most $Q_{\mathrm{diam}(B)+3 \mu}$ coarse $\mu$-connected components that meet $M \backslash \operatorname{Pen}(B, 2 \mu)$.

Proof. Fix an $\left(R, Q_{r}\right)$-quasi-lattice $\Gamma$ of $M$, and a point $x \in B$. By Corollary 6.11, the number of coarse $\mu$-connected components of $M \backslash B$ that meet $M \backslash \operatorname{Pen}(B, 2 \mu)$ is bounded by

$$
|\Gamma \cap \operatorname{Pen}(B, 3 \mu)| \leq|\Gamma \cap \bar{B}(x, \operatorname{diam}(B)+3 \mu)| \leq Q_{\operatorname{diam}(B)+3 \mu} .
$$

COROLlary 6.13. Suppose that $M$ is of coarse bounded geometry with coarse bound $\left(R, Q_{r}\right)$, and coarsely $\mu$-connected for some $\mu \geq R$. For any nonempty bounded subset $B \subset M$, let $C$ be the union of $B$ and the bounded coarse $\mu$-connected components of $M \backslash B$. Then $C$ is bounded, and the coarse $\mu$-connected components of $M \backslash C$ are the unbounded coarse $\mu$-connected components of $M \backslash B$.

PROOF. This is a consequence of Corollaries 6.12 and 6.8 .

Corollary 6.14. Suppose that $M$ is of coarse bounded geometry with coarse bound $\left(R, Q_{r}\right)$, coarsely $\mu$-connected for some $\mu \geq R$, and unbounded. Then the complement of each bounded subset of $M$ has an unbounded coarse $\mu$-connected component.

Proof. Take any bounded $B \subset M$. We can assume $B \neq \varnothing$ because $M$ is unbounded. Thus, if all coarse $\mu$-connected components of $M \backslash B$ were bounded, then $M$ would be bounded by Corollary 6.13 , a contradiction.

\subsection{Coarse ends}

Let $\mathcal{B}(M)$ (or simply $\mathcal{B}$ ) be the set of bounded subsets of $M$. For all $\mu>0$ and $B \in \mathcal{B}$, let $\mathcal{U}_{\mu, B}(M)$ (or simply $\mathcal{U}_{\mu, B}$ ) denote the discrete space of unbounded coarse $\mu$-connected components of $M \backslash B$. According to Remark 6.2-(ii), for $B \subset C$ in $\mathcal{B}$, we get a map $\eta_{\mu, B, C}: \mathcal{U}_{\mu, B} \rightarrow \mathcal{U}_{\mu, C}$ determined by $\eta_{\mu, B, C}(U) \supset U$. These spaces $\mathcal{U}_{\mu, B}$ and maps $\eta_{\mu, B, C}$ form a projective system (over $\mathcal{B}$ with " $\subset$ "), denoted by $\left\{\mathcal{U}_{\mu, B}, \eta_{\mu, B, C}\right\}$.

Definition 6.15. The projective limit of $\left\{\mathcal{U}_{\mu, B}, \eta_{\mu, B, C}\right\}$, denoted by $\mathcal{E}_{\mu}(M)$, is the space of $\mu$-ends of $M$.

REMARK 6.16. $\mathcal{E}_{\mu}(M)$ is Hausdorff and totally disconnected because each space $\mathcal{U}_{\mu, B}$ is discrete.

Each $\mathbf{e} \in \mathcal{E}_{\mu}(M)$ can be described as a map defined on $\mathcal{B}$ such that $\mathbf{e}(B) \in \mathcal{U}_{\mu, B}$ and $\mathbf{e}(B) \supset \mathbf{e}(C)$ if $B \subset C$. The maps $\eta_{\mu, B}: \mathcal{E}_{\mu}(M) \rightarrow \mathcal{U}_{\mu, B}$ satisfying the universal property of the inverse limit are given by $\eta_{\mu, B}(\mathbf{e})=\mathbf{e}(B)$. Hence, for $B \subset C$ in $\mathcal{B}$,

$$
\eta_{\mu, B, C}(\mathbf{e}(B))=\mathbf{e}(C) .
$$

Remark 6.17. We have $\mathbf{e}(B) \cap \mathbf{e}(C) \neq \varnothing$ for all $\mathbf{e} \in \mathcal{E}_{\mu}(M)$ and $B, C \in \mathcal{B}$, because $\mathbf{e}(B) \cap \mathbf{e}(C) \supset \mathbf{e}(B \cup C)$. 
For each $B \in \mathcal{B}$ and $U \in \mathcal{U}_{\mu, B}$, let

$$
\mathcal{N}_{\mu}(B, U)=\left\{\mathbf{e} \in \mathcal{E}_{\mu}(M) \mid \mathbf{e}(B)=U\right\} .
$$

The family of the sets $\mathcal{N}_{\mu}(B, U)$ is a base of the topology of $\mathcal{E}_{\mu}(M)$. For any fixed $x_{0} \in M$, an ultrametric $d_{\mu, x_{0}}$ inducing the topology of $\mathcal{E}_{\mu}(M)$ is given by

$$
d_{\mu, x_{0}}(\mathbf{e}, \mathbf{f})=\exp \left(-\sup \left\{n \in \mathbb{N} \mid \mathbf{e}\left(B\left(x_{0}, n\right)\right)=\mathbf{f}\left(B\left(x_{0}, n\right)\right)\right\}\right) .
$$

Remark 6.18. Since $\left\{B\left(x_{0}, n\right) \mid n \in \mathbb{N}\right\}$ is cofinal in $\mathcal{B}$, for each nested sequence $U_{0} \supset U_{1} \supset \cdots$ with $U_{n} \in \mathcal{U}_{\mu, B\left(x_{0}, n\right)}$, there is a unique $\mathbf{e} \in \mathcal{E}_{\mu}(M)$ such that $U_{n}=$ $\mathbf{e}\left(B\left(x_{0}, n\right)\right)$ for all $n$.

REMARK 6.19. The Lipschitz equivalence class of $d_{\mu, x_{0}}$ is independent of $x_{0}$; in fact, for another point $x_{1} \in M$ and an integer $N \geq d\left(x_{0}, x_{1}\right)$, we easily get $d_{\mu, x_{1}} \leq e^{N} d_{\mu, x_{0}}$.

According to Remark 6.2 (ii), for $0<\mu \leq \nu$ and $B \in \mathcal{B}$, there is a map $\theta_{\mu, \nu, B}$ : $\mathcal{U}_{\mu, B} \rightarrow \mathcal{U}_{\nu, B}$ determined by $\theta_{\mu, \nu, B}(U) \supset U$. The diagram

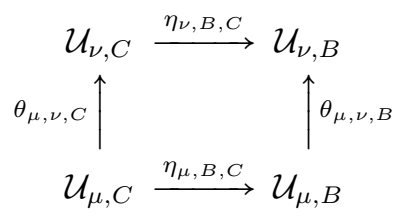

is commutative for $B \subset C$ in $\mathcal{B}$ because, for all $U \in \mathcal{U}_{\mu, B}$,

$$
\begin{gathered}
\eta_{\nu, B, C} \theta_{\mu, \nu, C}(U) \supset \theta_{\mu, \nu, C}(U) \supset U, \\
\theta_{\mu, \nu, B} \eta_{\mu, B, C} \supset \eta_{\mu, B, C}(U) \supset U .
\end{gathered}
$$

Hence the maps $\theta_{\mu, \nu, B}$ induce a continuous map $\theta_{\mu, \nu}: \mathcal{E}_{\mu}(M) \rightarrow \mathcal{E}_{\nu}(M)$ determined by the condition $\eta_{\nu, B} \theta_{\mu, \nu}=\theta_{\mu, \nu, B} \eta_{\mu, B}$ for all $B \in \mathcal{B}$. Observe that

$$
\theta_{\mu, \nu}(\mathbf{e})(B)=\eta_{\nu, B} \theta_{\mu, \nu}(\mathbf{e})=\theta_{\mu, \nu, B} \eta_{\mu, B}(\mathbf{e})=\theta_{\mu, \nu, B}(\mathbf{e}(B)) .
$$

On the other hand, like the commutativity of 6.2 , it can be proved that

$$
\theta_{\mu, \nu, B} \circ \theta_{\lambda, \mu, B}=\theta_{\lambda, \nu, B}
$$

for $0<\lambda \leq \mu \leq \nu$. Hence, by 6.3 ,

$$
\begin{aligned}
& \theta_{\mu, \nu} \theta_{\lambda, \mu}(\mathbf{e})(B)=\theta_{\mu, \nu, B}\left(\theta_{\lambda, \mu}(\mathbf{e})(B)\right)=\theta_{\mu, \nu, B} \theta_{\lambda, \mu, B}(\mathbf{e}(B)) \\
&= \theta_{\lambda, \nu, B}(\mathbf{e}(B))=\theta_{\lambda, \nu}(\mathbf{e})(B),
\end{aligned}
$$

giving $\theta_{\mu, \nu} \theta_{\lambda, \mu}=\theta_{\lambda, \nu}$. Thus the spaces $\mathcal{E}_{\nu}(M)$ and maps $\theta_{\mu, \nu}$ form a direct system of topological spaces, denoted by $\left\{\mathcal{E}_{\mu}(M), \theta_{\mu, \nu}\right\}$.

Definition 6.20. The injective limit of $\left\{\mathcal{E}_{\mu}(M), \theta_{\mu, \nu}\right\}$, denoted by $\mathcal{E}_{\infty}(M)$, is called the space of coarse ends of $M$.

Let $\theta_{\mu}: \mathcal{E}_{\mu}(M) \rightarrow \mathcal{E}_{\infty}(M)$ be the maps that satisfy the universal property of the injective limit.

REMARK 6.21. It is easy to see that

$$
\theta_{\mu, \nu}:\left(\mathcal{E}_{\mu}(M), d_{\mu, x_{0}}\right) \rightarrow\left(\mathcal{E}_{\nu}(M), d_{\nu, x_{0}}\right)
$$

is non-expanding for $\nu \geq \mu$. 
REMARK 6.22. The definition of the space of coarse ends can be generalized to arbitrary coarse spaces as follows. With the terminology of [86, for each entourage $E$ of a coarse space $X$, define the coarse $E$-connected components like the above coarse $\mu$-connected components by using the condition $(x, y) \in E$ instead of $d(x, y) \leq$ $\mu$. Let $\mathcal{B}$ be the family of bounded subsets of $X$ (those $B \subset X$ so that $B \times B$ is an entourage). Then we can define $\mathcal{U}_{E, B}$ and $\mathcal{E}_{E}(X)$ like the above $\mathcal{U}_{\mu, B}$ and $\mathcal{E}_{\mu}(M)$. For entourages $E \subset F$, we get a continuous map $\theta_{E, F}: \mathcal{E}_{E}(X) \rightarrow \mathcal{E}_{F}(X)$ defined like the above $\theta_{\mu, \nu}$ for $\mu \leq \nu$. Then $\left\{\mathcal{E}_{E}(X), \theta_{E, F}\right\}$ is a direct system whose injective limit is $\mathcal{E}_{\infty}(X)$.

REMARK 6.23. Observe that the spaces $\mathcal{E}_{\mu}(M)$ and $\mathcal{E}_{\infty}(M)$ are nonempty if and only if $M$ is unbounded.

Proposition 6.24. If $M$ is of coarse bounded geometry with coarse bound $\left(R, Q_{r}\right)$, and coarsely $\mu$-connected for some $\mu \geq R$, then $\mathcal{E}_{\mu}(X)$ is compact.

Proof. This holds because the spaces $\mathcal{U}_{\mu, B}$ are finite by Corollary 6.12.

Lemma 6.25. If $M$ is of coarse bounded geometry with coarse bound $\left(R, Q_{r}\right)$, and coarsely $\mu$-connected for some $\mu \geq R$, then $\eta_{\mu, B, C}$ is surjective for $\varnothing \neq B \subset C$ in $\mathcal{B}$.

Proof. Take any $U \in \mathcal{U}_{\mu, B}$. By Corollary 6.14, $U \backslash C$ has some unbounded coarse $\mu$-connected component $V$. Then $V \in \mathcal{U}_{\mu, C}$ by Lemma 6.7, and $\eta_{\mu, B, C}(V)=$ $U$.

COROLlaRY 6.26. If $M$ is of coarse bounded geometry with coarse bound $\left(R, Q_{r}\right)$, and coarsely $\mu$-connected for some $\mu \geq R$, then $\eta_{\mu, B}$ is surjective for $\varnothing \neq B \in \mathcal{B}$.

Proof. By inductively applying Lemma 6.25, it follows that for every $U \in \mathcal{U}_{\mu, B}$ there is a nested sequence $U=U_{0} \supset U_{1} \supset \cdots$ such that $U_{n} \in \mathcal{U}_{\mu, \operatorname{Pen}(B, n)}$ for all $n \in \mathbb{N}$. Since $\{\operatorname{Pen}(B, n) \mid n \in \mathbb{N}\}$ is cofinal in $\mathcal{B}$, there is a unique $\mathbf{e} \in \mathcal{E}_{\mu}(M)$ such that $\mathbf{e}(\operatorname{Pen}(B, n))=U_{n}$ for all $n \in \mathbb{N}$; in particular, $U=\mathbf{e}(B)=\eta_{\mu, B}(\mathbf{e})$.

LEMMA 6.27. If $M$ is of coarse bounded geometry with coarse bound $\left(R, Q_{r}\right)$, and coarsely $\mu$-connected for some $\mu \geq R$, then $\theta_{\mu, \nu, B}$ is surjective for nonempty sets $B \in \mathcal{B}$ and $\nu \geq \mu>0$.

Proof. Every $V \in \mathcal{U}_{\nu, B}$ is union of coarse $\mu$-connected components of $M \backslash B$ (Remark 6.2-(ii)). Moreover $M \backslash B$ has a finite number of coarse $\mu$-connected components that meet $M \backslash \operatorname{Pen}(B, 2 \mu)$ by Corollary 6.12 . Since $V$ is unbounded, it follows that $V$ contains some unbounded coarse $\mu$-connected component $U$ of $M \backslash B$. Thus $U \in \mathcal{U}_{\mu, B}$ and $\theta_{\mu, \nu, B}(U)=V$.

LEMMA 6.28. If $M$ is of coarse bounded geometry with coarse bound $\left(R, Q_{r}\right)$, and coarsely $\mu$-connected for some $\mu \geq R$, then $\theta_{\mu, \nu}: \mathcal{E}_{\mu}(M) \rightarrow \mathcal{E}_{\nu}(M)$ has dense image for $\nu \geq \mu$.

Proof. This follows from Corollary 6.26, Lemma 6.27 and [39, Appendix Two, Theorem 2.5-(2), p. 430].

Proposition 6.29. If $M$ is of coarse bounded geometry and coarsely connected, then $\mathcal{E}_{\infty}(M)$ is compact. 
Proof. $\mathcal{E}_{\mu}(M)$ is compact for $\mu$ large enough by Proposition 6.24. Moreover it is also Hausdorff. So $\theta_{\mu, \nu}$ is surjective for $\mu$ large enough and $\nu \geq \mu$ by Lemma 6.28, obtaining that $\theta_{\mu}: \mathcal{E}_{\mu}(M) \rightarrow \mathcal{E}_{\infty}(M)$ is surjective. Therefore $\mathcal{E}_{\infty}(M)$ is compact.

Lemma 6.30. (i) Suppose that $M$ is the metric space of vertices of a connected graph. For $B \in \mathcal{B}$ and $N \in \mathbb{Z}^{+}$, let $\widetilde{B}=\operatorname{Pen}\left(B,\left\lceil\frac{N-1}{2}\right\rceil\right)$. Then every coarse $N$-connected component of $M \backslash \widetilde{B}$ is contained in some coarse 1-connected component of $M \backslash B$.

(ii) Assume that $M$ is a connected complete Riemannian manifold 1 , For every closed $B \in \mathcal{B}$ and $\mu, \varepsilon>0$, let $\widetilde{B}=\operatorname{Pen}\left(B, \frac{\mu+\varepsilon}{2}\right)$. Then every coarse $\mu$-connected component of $M \backslash \widetilde{B}$ is contained in some connected component of $M \backslash B$.

Proof. (i) Let $U$ be a coarse $N$-connected component of $M \backslash \widetilde{B}$. For $x, y \in U$, there are points $x=z_{0}, z_{1}, \ldots, z_{k}=y$ in $U$ such that $d\left(z_{l-1}, z_{l}\right) \leq N$ for all $l \in$ $\{1, \ldots, k\}$. Then there are points, $z_{l-1}=u_{0}^{l}, u_{1}^{l}, \ldots, u_{p_{l}}^{l}=z_{l}$, in $M$ such that $p_{l} \leq N$ and $d\left(u_{q-1}^{l}, u_{q}^{l}\right)=1$ for all $q \in\left\{1, \ldots, p_{l}\right\}$. Observe that $u_{q}^{l} \in \operatorname{Pen}\left(U,\left\lceil\frac{N-1}{2}\right\rceil\right) \subset M \backslash B$. So $x$ is coarsely 1-connected to $y$ in $M \backslash B$. It follows that $U$ is a coarsely 1connected subset of $M \backslash B$, and therefore it is contained in some coarsely 1-connected component of $M \backslash B$.

(ii) For $x, y \in U \in \mathcal{U}_{\mu, \widetilde{B}}$, there are points $x=z_{0}, z_{1}, \ldots, z_{k}=y$ in $U$ such that $d\left(z_{l-1}, z_{l}\right) \leq \mu$ for all $l \in\{1, \ldots, k\}$. Thus there is a smooth path $\alpha_{l}$ from $z_{l-1}$ to $z_{l}$ with length $<\mu+\varepsilon$. Each $\alpha_{l}$ is a path in $\operatorname{Pen}\left(U, \frac{\mu+\varepsilon}{2}\right) \subset M \backslash B$, and so the product path $\alpha_{1} \cdots \alpha_{k}$ joins $x$ to $y$ in $M \backslash B$. Thus $U$ is a connected subset of $M \backslash B$, and hence it is contained in some connected component of $M \backslash B$.

Proposition 6.31. If $M$ is the metric space of vertices of a connected graph $G$ that has finitely many edges abutting on each vertex, then $\mathcal{E}(G) \equiv \mathcal{E}_{1}(M) \approx \mathcal{E}_{\infty}(M)$, canonically.

Proof. The definitions of $\mathcal{E}_{1}(M)$ and $\mathcal{E}(G)$ are canonically equivalent so it has to be shown that $\mathcal{E}_{1}(M) \approx \mathcal{E}_{\infty}(M)$, canonically, which will be a consequence of showing that $\theta_{1, N}$ is a homeomorphism for each $N \in \mathbb{Z}^{+}$. For every $B \in \mathcal{B}$, let $\widetilde{B}=\operatorname{Pen}\left(B,\left\lceil\frac{N-1}{2}\right\rceil\right)$. By Lemma 6.30-(i), a map $\xi_{N, B}: \mathcal{U}_{N, \widetilde{B}} \rightarrow \mathcal{U}_{1, B}$ is determined by $\xi_{N, B}(U) \supset U$. Like in the case of (6.2), it can be easily checked that, for $B \subset C$ in $\mathcal{B}$, the diagrams

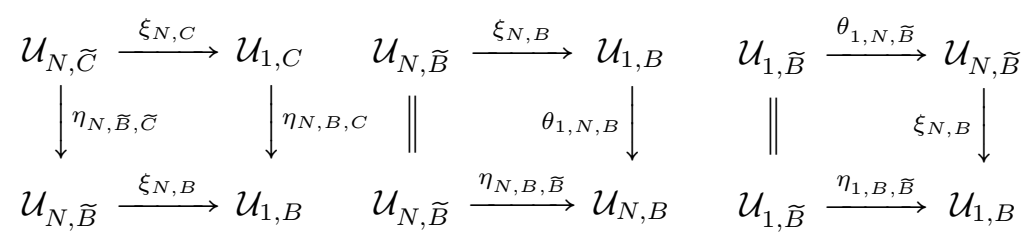

are commutative. So the maps $\xi_{N, B}$ induce a continuous map $\xi_{N}: \mathcal{E}_{N}(M) \rightarrow \mathcal{E}_{1}(M)$ that is inverse of $\theta_{1, N}$. Thus $\theta_{1, N}$ is a homeomorphism.

Proposition 6.32. If $M$ is a connected complete Riemannian manifold, then $\mathcal{E}(M) \approx \mathcal{E}_{\mu}(M) \approx \mathcal{E}_{\infty}(M)$, canonically, for all $\mu>0$.

\footnotetext{
${ }^{1}$ In fact, the proof of this property applies to any complete path metric space, as well as the
} proof of Proposition 6.32 

(iii).

Proof. The proof is similar to that of Proposition 6.31, using Lemma 6.30

\subsection{Functoriality of the space of coarse ends}

Let $f: M \rightarrow M^{\prime}$ be a coarse map; in particular, it satisfies the condition of uniform expansiveness with some mapping $s_{r}$, which can be assumed to be nondecreasing. We have $f^{-1}\left(B^{\prime}\right) \in \mathcal{B}(M)$ for all $B^{\prime} \in \mathcal{B}\left(M^{\prime}\right)$ because $f$ is metric

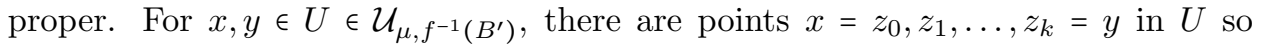
that $d\left(z_{l-1}, z_{l}\right) \leq \mu$ for $l \in\{1, \ldots, k\}$. Since $d^{\prime}\left(f\left(z_{l}\right), f\left(z_{l-1}\right)\right) \leq s_{\mu}$, we get that $f(U)$ is a coarsely $s_{\mu}$-connected subset of $M^{\prime} \backslash B^{\prime}$. Hence $f(U)$ is contained in some coarse $s_{\mu}$-connected component $U^{\prime}$ of $M^{\prime} \backslash B^{\prime}$. Moreover $U^{\prime}$ is unbounded; otherwise, $f^{-1}(f(U))$ is bounded because $f$ is metrically proper, obtaining that $U$ is bounded, a contradiction. Thus there is a map $f_{\mu, B^{\prime}}: \mathcal{U}_{\mu, f^{-1}\left(B^{\prime}\right)}(M) \rightarrow \mathcal{U}_{s_{\mu}, B^{\prime}}\left(M^{\prime}\right)$ determined by $f_{\mu, B^{\prime}}(U) \supset f(U)$. Since $B \subset f^{-1}(f(B))$ for all $B \in \mathcal{B}(M)$, and $f(B) \in \mathcal{B}\left(M^{\prime}\right)$ by the uniform expansiveness of $f$, the set $\left\{f^{-1}\left(B^{\prime}\right) \mid B^{\prime} \in \mathcal{B}\left(M^{\prime}\right)\right\}$ is cofinal in $\mathcal{B}$. Hence the maps $f_{\mu, B^{\prime}}$ induce a continuous map $f_{\mu}: \mathcal{E}_{\mu}(M) \rightarrow \mathcal{E}_{s_{\mu}}\left(M^{\prime}\right)$, determined by the condition $\eta_{\mu, B^{\prime}} \circ f_{\mu}=f_{\mu, B^{\prime}} \circ \eta_{\mu, f^{-1}\left(B^{\prime}\right)}$ for all $B^{\prime} \in \mathcal{B}\left(M^{\prime}\right)$. Thus

$$
f_{\mu}(\mathbf{e})\left(B^{\prime}\right)=\eta_{\mu, B^{\prime}} \circ f_{\mu}(\mathbf{e})=f_{\mu, B^{\prime}} \circ \eta_{\mu, f^{-1}\left(B^{\prime}\right)}(\mathbf{e})=f_{\mu, B^{\prime}}\left(\mathbf{e}\left(f^{-1}\left(B^{\prime}\right)\right)\right)
$$

for all $B^{\prime} \in \mathcal{B}\left(M^{\prime}\right)$. As in the case of 6.2 , it is easy to check that the diagram

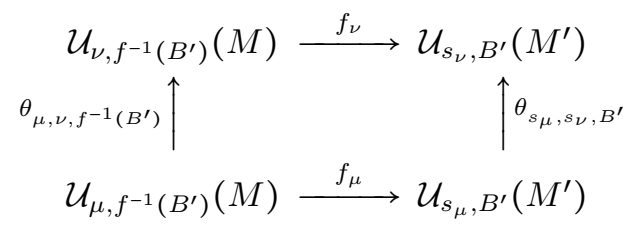

is commutative for $0<\mu<\nu$, and thus the diagram

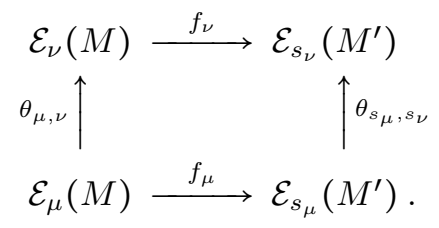

is also commutative. So the maps $f_{\mu}$ induce a continuous map $f_{\infty}: \mathcal{E}_{\infty}(M) \rightarrow$ $\mathcal{E}_{\infty}\left(M^{\prime}\right)$

Lemma 6.33. $f_{\infty}$ is independent of the choice of $s_{r}$.

Proof. Let $\bar{s}_{r} \geq s_{r}$ for each $r \geq 0$, and let

$$
\begin{gathered}
\bar{f}_{\mu, B^{\prime}}: \mathcal{U}_{\mu, f^{-1}\left(B^{\prime}\right)}(M) \rightarrow \mathcal{U}_{\bar{s}_{\mu}, B^{\prime}}\left(M^{\prime}\right), \\
\bar{f}_{\mu}: \mathcal{E}_{\mu}(M) \rightarrow \mathcal{E}_{\bar{s}_{\mu}}\left(M^{\prime}\right), \quad \bar{f}_{\infty}: \mathcal{E}_{\infty}(M) \rightarrow \mathcal{E}_{\infty}\left(M^{\prime}\right)
\end{gathered}
$$

be the maps induced by $f$ and $\bar{s}_{r}$. For $\mu>0$ and $B^{\prime} \in \mathcal{B}\left(M^{\prime}\right)$, the commutativity of the diagram

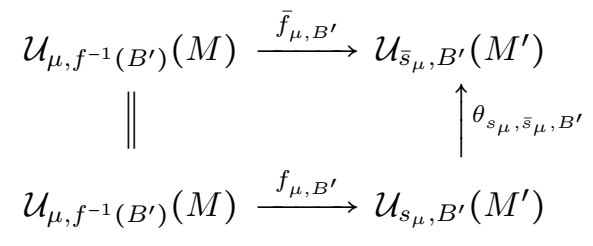


follows like in the case of 6.2 , obtaining the commutativity of

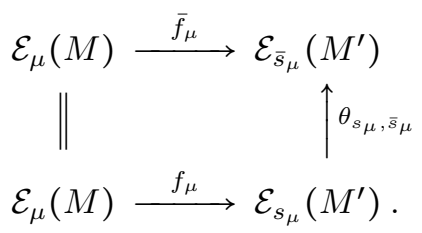

Therefore $\bar{f}_{\infty}=f_{\infty}$.

Proposition 6.34. If $g: M \rightarrow M^{\prime}$ is another coarse map close to $f$, then $g_{\infty}=f_{\infty}$.

Proof. Take some $c \geq 0$ such that $f$ and $g$ are $c$-close. We can suppose that $g$ also satisfies the condition of uniform expansiveness with $s_{r}$. Let $\mu>0$, $B \in \mathcal{B}(M), B^{\prime} \in \mathcal{B}\left(M^{\prime}\right), U \in \mathcal{U}_{\mu, B}(M), V \in \mathcal{U}_{\mu, f^{-1}\left(B^{\prime}\right)}(M)$ and $W \in \mathcal{U}_{\mu, g^{-1}\left(B^{\prime}\right)}(M)$ such that $f^{-1}\left(B^{\prime}\right) \cup g^{-1}\left(B^{\prime}\right) \subset B$ and $U \subset V \cap W$. Therefore $\eta_{\mu, f^{-1}\left(B^{\prime}\right), B}(U)=V$ and $\eta_{\mu, g^{-1}\left(B^{\prime}\right), B}(U)=W$. We know that $f(V)$ and $g(W)$ are coarsely $s_{\mu}$-connected subsets of $M^{\prime} \backslash B^{\prime}$. By Remark 6.2 (iv) and since $d^{\prime}(f(x), g(x)) \leq c$ for all $x \in U$, it follows that $f(V) \cup g(W)$ is a coarsely $\bar{s}_{\mu}$-connected subset of $M^{\prime} \backslash B^{\prime}$, where $\bar{s}_{\mu}=\max \left\{s_{\mu}, c\right\}$. Hence $f(V)$ and $g(W)$ are contained in the same coarse $\bar{s}_{\mu^{-}}$ connected component of $M^{\prime} \backslash B^{\prime}$. This shows that the diagram

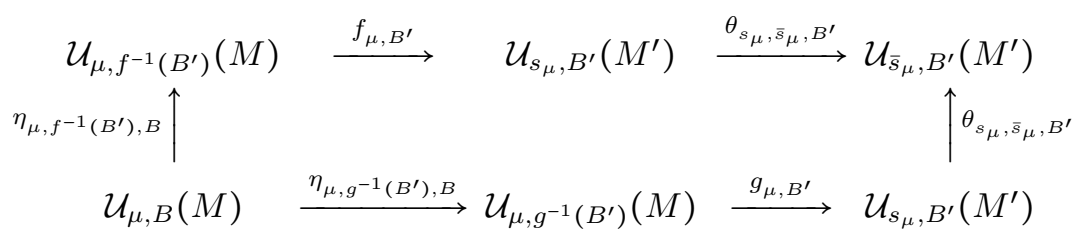

is commutative. So the diagram

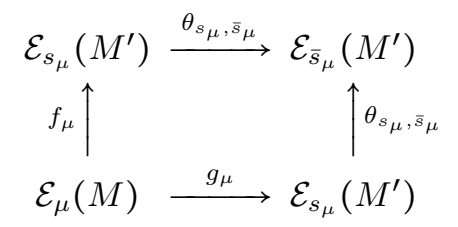

is also commutative, and $f_{\infty}=g_{\infty}$ obtains.

Proposition 6.35. A functor $\mathcal{E}_{\infty}$ of the metric coarse category to the category of continuous maps between topological spaces is defined by $\mathcal{E}_{\infty}([M])=\mathcal{E}_{\infty}(M)$ and $\mathcal{E}_{\infty}([f])=f_{\infty}$.

Proof. Obviously, $\left(\operatorname{id}_{M}\right)_{\mu}=\operatorname{id}_{\mathcal{E}_{\mu}(M)}$ for all $\mu>0$, and therefore $\left(\operatorname{id}_{M}\right)_{\infty}=$ $\operatorname{id}_{\mathcal{E}_{\infty}(M)}$. Suppose that $f^{\prime}: M^{\prime} \rightarrow M^{\prime \prime}$ satisfies the condition of uniform expansiveness with a mapping $s_{r}^{\prime}$. Then $f^{\prime} f$ satisfies the condition of uniform expansiveness with the mapping $s_{s_{r}}^{\prime}$, and, like in the case of 6.2, we get that the composite

$$
\mathcal{U}_{\mu, f^{-1}\left(f^{\prime-1}\left(B^{\prime \prime}\right)\right)}(M) \stackrel{f_{\mu, f^{\prime-1}\left(B^{\prime \prime}\right)}^{\longrightarrow}}{\longrightarrow} \mathcal{U}_{s_{\mu}, f^{\prime-1}\left(B^{\prime \prime}\right)}\left(M^{\prime}\right) \stackrel{f_{s_{\mu}, B^{\prime \prime}}^{\prime}}{\longrightarrow} \mathcal{U}_{s_{s_{\mu}}^{\prime}, B^{\prime \prime}}\left(M^{\prime \prime}\right)
$$

equals $\left(f^{\prime} f\right)_{\mu, B^{\prime \prime}}$ for all $\mu>0$ and $B^{\prime \prime} \in \mathcal{B}\left(M^{\prime \prime}\right)$. So the composite

$$
\mathcal{E}_{\mu}(M) \stackrel{f_{\mu}}{\longrightarrow} \mathcal{E}_{s_{\mu}}\left(M^{\prime}\right) \stackrel{f_{s_{\mu}}^{\prime}}{\longrightarrow} \mathcal{E}_{s_{s_{\mu}}^{\prime}}\left(M^{\prime \prime}\right)
$$


equals $\left(f^{\prime} f\right)_{\mu}$ for all $\mu>0$, obtaining $f_{\infty}^{\prime} f_{\infty}=\left(f^{\prime} f\right)_{\infty}$. This shows that $M \mapsto \mathcal{E}_{\infty}(M)$ and $f \mapsto f_{\infty}$ defines a functor of the category of coarse maps between metric spaces to the category of continuous maps between topological spaces. Then $\mathcal{E}_{\infty}(M)$ depends only on $[M]$. Furthermore $f_{\infty}$ depends only on $[f]$ by Lemma 6.33 and Proposition 6.34.

Remark 6.36. Continuing with the ideas of Remark 6.22, the functor $\mathcal{E}_{\infty}$ can be obviously extended to the whole coarse category.

COROLlary 6.37. If $f: M \rightarrow M^{\prime}$ is a coarse equivalence, then $f_{\infty}$ is a homeomorphism.

Corollary 6.38. The spaces of ends of two coarsely quasi-isometric metric spaces are homeomorphic.

\subsection{Coarse end space of a class of metric spaces}

The following result extends a well known theorem for finitely generated groups (Example 3.1) [48, Theorem 13.5.7].

THEOREM 6.39. Assume that $M$ is of coarse bounded geometry, coarsely quasiconvex and coarsely quasi-symmetric. Then, either $\left|\mathcal{E}_{\infty}(M)\right| \leq 2$, or $\mathcal{E}_{\infty}(M)$ is a Cantor space.

Proof. By Corollaries 3.12 and 6.38, Propositions 3.19 and 3.25 , and Example 3.7. we can assume that $M$ is the metric space of vertices of a connected graph of finite type. Thus $\mathcal{E}_{1}(M) \approx \mathcal{E}_{\infty}(M)$ by Proposition 6.31. Suppose that $\left|\mathcal{E}_{1}(M)\right| \geq 3$, and let us prove that $\mathcal{E}_{1}(M)$ is a Cantor space. Take three distinct elements $\mathbf{e}_{k} \in \mathcal{E}_{1}(M), k \in\{1,2,3\}$. Since $\mathcal{E}_{1}(M)$ is Hausdorff, totally disconnected and compact (Propositions 6.29 and 6.31), it is enough to prove that $\left|\mathcal{N}_{1}(B, U)\right| \geq 2$ for any basic open set $\mathcal{N}_{1}(B, U)$ of $\mathcal{E}_{1}(M)$ (Section 6.3). We can assume that $B$ is coarsely 1-connected and the sets $\mathbf{e}_{k}(B)$ are distinct.

By Propositions 2.26 and 2.39, there is a transitive set $\mathcal{T}$ of equi-rough transformations of $M$, and let $\left(s_{r}, c\right)$ be a common rough equivalence distortion of all maps in $\mathcal{T}$. We can suppose that $s_{r} \uparrow \infty$ as $r \rightarrow \infty$, and $s_{n}, c \in \mathbb{N}$ for all $n \in \mathbb{N}$. Let $N=\max \left\{s_{s_{1}}, c\right\} \geq 1$ and $\widetilde{B}=\operatorname{Pen}\left(B,\left\lceil\frac{N-1}{2}\right\rceil\right)$.

Claim 4. For each $f \in \mathcal{T}$, the sets $f_{1}\left(\mathbf{e}_{k}\right)(\operatorname{Pen}(f(\widetilde{B}), c))$ are distinct.

Given any $f \in \mathcal{T}$, let $g: M \rightarrow M$ be a rough equivalence with rough distortion $s_{r}$ such that $g f$ and $f g$ are $c$-close to id ${ }_{M}$. Let $C=\operatorname{Pen}(\widetilde{B}, c)$. We have $(g f)^{-1}(\widetilde{B}) \subset C$ because, if $x \in(g f)^{-1}(\widetilde{B})$, then $g f(x) \in \widetilde{B}$ and $d(x, g f(x)) \leq c$. Then the diagram

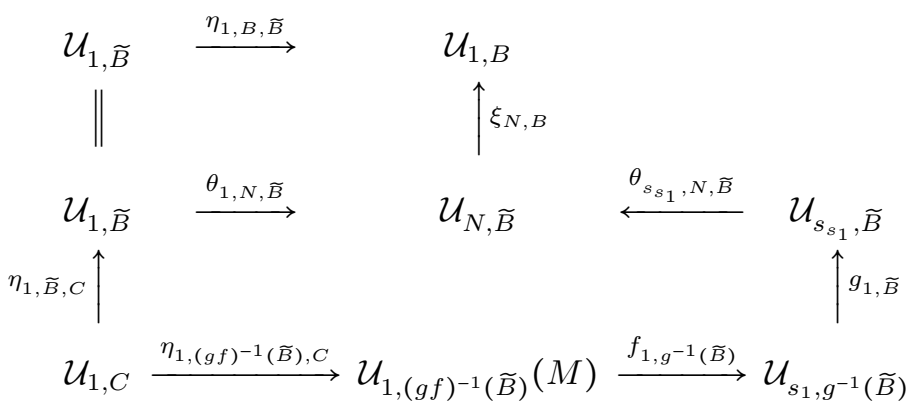


is commutative according to the proofs of Propositions 6.31, 6.34 and 6.35 Hence, by 6.1,

$$
\begin{aligned}
\xi_{N, B} \circ \theta_{s_{s_{1}}, N, \widetilde{B}} \circ g_{1, \widetilde{B}} \circ f_{1, g^{-1}(\widetilde{B})} \circ \eta_{1,(g f)^{-1}(\widetilde{B}), C}\left(\mathbf{e}_{k}(C)\right) \\
=\xi_{N, B} \circ \theta_{1, N, \widetilde{B}} \circ \eta_{1, \widetilde{B}, C}\left(\mathbf{e}_{k}(C)\right)=\xi_{N, B} \circ \theta_{1, N, \widetilde{B}}\left(\mathbf{e}_{k}(\widetilde{B})\right) \\
=\eta_{1, B, \widetilde{B}}\left(\mathbf{e}_{k}(\widetilde{B})\right)=\mathbf{e}_{k}(B),
\end{aligned}
$$

which are distinct sets for $k \in\{1,2,3\}$. Thus the sets

$$
\begin{aligned}
f_{1, g^{-1}(\widetilde{B})} \circ \eta_{1,(g f)^{-1}(\widetilde{B}), C}\left(\mathbf{e}_{k}(C)\right) & =f_{1,(g f)^{-1}(\widetilde{B})}\left(\mathbf{e}_{k}\left((g f)^{-1}(\widetilde{B})\right)\right) \\
& =f_{1}\left(\mathbf{e}_{k}\right)\left(\mathbf{e}_{k}\left(g^{-1}(\widetilde{B})\right)\right)
\end{aligned}
$$

are also distinct, where we have used (6.1) and (6.5). On the other hand, $g^{-1}(\widetilde{B}) \subset$ $\operatorname{Pen}(f(\widetilde{B}), c)$ because, if $x \in g^{-1}(\widetilde{B})$, then $f g(x) \in f(\widetilde{B})$ and $d(f g(x), x) \leq c$. Then Claim 4 follows because

$$
f_{1}\left(\mathbf{e}_{k}\right)\left(\mathbf{e}_{k}\left(g^{-1}(\widetilde{B})\right)\right)=\eta_{1, g^{-1}(\widetilde{B}), \operatorname{Pen}(f(\widetilde{B}), c)} \circ f_{1}\left(\mathbf{e}_{k}\right)\left(\mathbf{e}_{k}(\operatorname{Pen}(f(\widetilde{B}), c))\right) .
$$

Take any integer $R \geq \operatorname{diam} B$. Since $U$ is unbounded and $\mathcal{T}$ transitive, we can fix some $f \in \mathcal{T}$ such that there is some $x \in U \cap f(\widetilde{B})$ with

$$
d(x, B)>c+s_{R+N}+N+1 .
$$

Since $\operatorname{diam} \widetilde{B} \leq R+N$, we have $\operatorname{diam} f(\widetilde{B}) \leq s_{R+N}$, obtaining

$$
d(f(\widetilde{B}), B)>c+N+1 .
$$

Let

$$
\begin{gathered}
B^{\prime}=\operatorname{Pen}(f(\widetilde{B}), c), \quad B^{\prime \prime}=\operatorname{Pen}\left(f(\widetilde{B}), c+\left\lceil\frac{N-1}{2}\right\rceil\right), \\
V_{k}=f_{1}\left(\mathbf{e}_{k}\right)\left(B^{\prime}\right) \in \mathcal{U}_{s_{1}, B^{\prime}}, \quad W_{k}=f_{1}\left(\mathbf{e}_{k}\right)\left(B^{\prime \prime}\right) \in \mathcal{U}_{s_{1}, B^{\prime \prime}} .
\end{gathered}
$$

Using the notation of the proof of Proposition 6.31, the composite

$$
\mathcal{U}_{s_{1}, B^{\prime \prime}} \stackrel{\theta_{s_{1}, N, B^{\prime \prime}}}{\longrightarrow} \mathcal{U}_{N, B^{\prime \prime}} \stackrel{\xi_{N, B^{\prime}}}{\longrightarrow} \mathcal{U}_{1, B^{\prime}}
$$

is defined because $N \geq s_{1}$. So each $W_{k}$ is contained in some $U_{k}^{\prime} \in \mathcal{U}_{1, B^{\prime}}$. Moreover $U_{k}^{\prime} \subset V_{k}$ by Remark 6.2 (ii) because $W_{k} \subset V_{k}$; in particular, the sets $U_{k}^{\prime}$ are disjoint from each other by Claim 4 .

Claim 5. $U$ meets all sets $U_{k}^{\prime}$.

Since $B$ is coarsely 1 -connected, $\operatorname{Pen}\left(B^{\prime \prime}, 1\right)$ is coarsely $s_{1}$-connected. Moreover $\operatorname{Pen}\left(B^{\prime \prime}, 1\right)$ is disjoint from $\widetilde{B}$ by $(6.6)$. So $\operatorname{Pen}\left(B^{\prime \prime}, 1\right)$ is contained in some coarse 1-connected component of $M \backslash B$ by Lemma 6.30 (i) and Remark 6.2 (i) because $N \geq s_{1}$. Since $f(B)$ meets $U$, we get $\operatorname{Pen}\left(B^{\prime \prime}, 1\right) \subset U$. So $U$ meets every $W_{k}$ by Lemma 6.9 and therefore it also meets every $U_{k}^{\prime}$, showing Claim 5 .

Claim 6. $\widetilde{B}$ meets at most one of the sets $U_{k}^{\prime}$.

Indeed, suppose that $\widetilde{B}$ meets two of the sets $U_{k}^{\prime}$, say $U_{1}^{\prime}$ and $U_{2}^{\prime}$. Since $U_{1}^{\prime}, U_{2}^{\prime} \epsilon$ $\mathcal{U}_{1, B^{\prime}}$ and $\widetilde{B}$ is coarsely 1-connected and disjoint from $B^{\prime}$ by $(6.6)$, it follows that $U_{1}^{\prime} \cup U_{2}^{\prime} \cup \widetilde{B}$ is an unbounded coarsely 1-connected subset of $M \backslash B^{\prime}$. Therefore $U_{1}^{\prime}=U_{2}^{\prime}$, a contradiction, confirming Claim 6 . 
According to Claim 6, assume from now on that $U_{1}^{\prime}, U_{2}^{\prime} \subset M \backslash \widetilde{B}$. Since these subsets are coarsely 1-connected, they are contained in coarse 1-connected components of $M \backslash B$ (Remark 6.2 (ii)). Then $U_{1}^{\prime}, U_{2}^{\prime} \subset U$ by Claim 5 By Corollary 6.8. $U_{1}^{\prime}, U_{2}^{\prime} \in \mathcal{U}_{1, B \cup B^{\prime}}$, and, by Corollary 6.26, there are $\mathbf{e}_{1}^{\prime}, \mathbf{e}_{2}^{\prime} \in \mathcal{E}_{1}(M)$ such that $\mathbf{e}_{k}^{\prime}\left(B \cup B^{\prime}\right)=U_{k}^{\prime}$ for $k \in\{1,2\}$. So $\mathbf{e}_{1}^{\prime} \neq \mathbf{e}_{2}^{\prime}$ and $\mathbf{e}_{k}^{\prime}(B) \supset \mathbf{e}_{k}^{\prime}\left(B \cup B^{\prime}\right)=U_{k}^{\prime}$, obtaining $\mathbf{e}_{k}^{\prime}(B)=U$ because $U_{k}^{\prime} \subset U$. Thus $\mathbf{e}_{1}^{\prime}, \mathbf{e}_{2}^{\prime} \in \mathcal{N}_{1}(B, U)$, showing that $\left|\mathcal{N}_{1}(B, U)\right| \geq 2$. 



\section{CHAPTER 7}

\section{Higson corona and asymptotic dimension}

The Higson corona of any coarse space is very important in coarse geometry; roughly speaking, it contains all coarse information. Even though it is a very vast space, some of our main results state that the Higson corona of leaves has some nice properties, Theorems 1.11 and 1.141 .16 .

The asymptotic dimension is also recalled in this chapter. It is strongly related to the topological dimension of the Higson corona, as indicated in Chapter 1 . Our main result Theorem 1.12 is about the asymptotic dimension of leaves.

\subsection{Compactifications}

Recall that a compactification of a topological space $X$ is a pair $(Y, h)$ consisting of a compact Hausdorff ${ }^{1}$ space $Y$ and an embedding $h: X \rightarrow Y$ with dense image. The subspace $Y \backslash h(X) \subset Y$ is called the corona of the compactification. Usually, the notation is simplified by assuming that $X \subset Y$ and $h$ is the inclusion map, which is omitted from the notation; in particular, it will be simply said that $X$ is open in $Y$ when $h$ is an open map. A typical notation is $\bar{X}$ for a compactification and $\partial X$ for the corona, or $X^{\gamma}$ for the compactification and $\gamma X$ for the corona, specially when $\gamma$ refers to some kind of compactification of a class of spaces.

The space $X$ admits a compactification if and only if it is Tychonov. Moreover $X$ is open in some compactification if and only if it is locally compact and Hausdorff; in this case, $X$ is open in any compactification.

Two compactifications of $X,(Y, h)$ and $\left(Y^{\prime}, h^{\prime}\right)$, are equivalent when there is a homeomorphism $\phi: Y^{\prime} \rightarrow Y$ so that $\phi h^{\prime}=h$. The term "compactification" will refer to an equivalence classes of compactifications. In this sense, the set ${ }^{2}$ of compactifications has a partial order relation, "s", defined by declaring $(Y, h) \leq$ $\left(Y^{\prime}, h^{\prime}\right)$ if there is a continuous $\pi: Y^{\prime} \rightarrow Y$ so that $\pi h^{\prime}=h$.

For a locally compact Hausdorff space $X$, let $C_{b}(X)$ denote the commutative $C^{*}$ algebra of bounded $\mathbb{C}$-valued continuous functions on $X$ with the supremum norm, and let $C_{0}(X) \subset C_{b}(X)$ be the closed involutive ideal of continuous functions that vanish at infinity ${ }^{3}$. The closed subalgebra of constant functions on $X$ may be canonicaly identified to $\mathbb{C}$.

The Gelfand-Naimark theorem estates that the assignment $X \mapsto C_{b}(X)$ defines a one-to-one correspondence between the (homeomorphism classes of) compact Hausdorff spaces and the (isomorphism classes of) commutative $C^{*}$ algebras with unit. The compact Hausdorff space $\Delta(\mathcal{A})$ that corresponds to each unital

\footnotetext{
${ }^{1}$ We only consider Hausdorff compactifications.

${ }^{2}$ All compactifications of $X$ are $\leq X^{\beta}$, where $X^{\beta}$ is the Stone-Čech compactification. Thus we can assume that they are quotients of $X^{\beta}$, and therefore they form a set.

${ }^{3}$ Recall that a function $f: X \rightarrow \mathbb{C}$ vanishes at infinity when, for all $\varepsilon>0$, there is a compact $K \subset X$ so that $|f(x)|<\varepsilon$ for all $x \in X \backslash K$.
} 
commutative $C^{*}$ algebra $\mathcal{A}$ is the space of characters $\mathcal{A} \rightarrow \mathbb{C}$ with the topology of pointwise convergence. For a compact Hausdorff space $X$, a canonical homeomorphism $h: X \rightarrow \Delta\left(C_{b}(X)\right)$ is given by evaluation: $h(x)(f)=f(x)$ for all $x \in X$ and $f \in C_{b}(X)$.

More generally, when $X$ is a locally compact Hausdorff space $X$, the assignment $(Y, h) \mapsto h^{*} C(Y)$ is a one-to-one correspondence between (equivalence classes) of Hausdorff compactifications of $X$ and unital closed involutive subalgebras of $C_{b}(X)$ that generate the topology (in the sense that compact sets can be separated from points by functions in the algebra). For each subalgebra $\mathcal{A} \subset C(X)$ of the above type, the corresponding compactification is $(\Delta(\mathcal{A}), h)$, where $h: X \rightarrow \Delta(\mathcal{A})$ is defined by evaluation at each point of $X$, as before. For instance, $C_{b}(X)$ correponds to the Stone-Čech compactification $X^{\beta}$, and $\mathbb{C}+C_{0}(X)$ corresponds to the one-point compactification $X^{*}$.

ExAmple 7.1. Consider the notation of Section 6.1. If $M$ is of coarse bounded geometry and coarsely connected, then $\mathcal{E}_{\infty}(M)$ is compact (Proposition 6.29). If moreover $M$ is proper, then $\mathcal{E}_{\infty}(M)$ is the corona of a compactification of $\overline{M \text {, which }}$ can be seen as follows. To show this property, let us prove first that $\mathcal{E}_{\mu}(M)$ is the corona of a compactification of $M$ for $\mu>0$ large enough, like the usual space of ends of a manifold or a graph. The space $\mathcal{E}_{\mu}(M)$ is compact for $\mu>0$ large enough (Proposition 6.24). Then let $\bar{M}_{\mu}=M \cup \mathcal{E}_{\mu}(M)$ with the topology so that the inclusion map $M \hookrightarrow \bar{M}_{\mu}$ is an open embedding, and a base of neighborhoods in $\bar{M}_{\mu}$ of any $\mathbf{e} \in \mathcal{E}_{\mu}(M)$ is given by the sets $\mathbf{e}(B)$ for $B \in \mathcal{B}(M)$. Using Corollary 6.12, it can be easily seen that $\bar{M}_{\mu}$ is compact, and it is obvious that $M$ is dense in $\bar{M}_{\mu}$. Now, for $\mu<\nu$ large enough, the continuous map $\theta_{\mu, \nu}: \mathcal{E}_{\mu}(M) \rightarrow \mathcal{E}_{\nu}(M)$ is surjective because it has dense image (Lemma 6.28) and these spaces are compact and Hausdorff. The combination of $\theta_{\mu, \nu}$ and $\mathrm{id}_{M}$, denoted by $\bar{\theta}_{\mu, \nu}: \bar{M}_{\mu} \rightarrow \bar{M}_{\nu}$, is continuous by 6.3 and the definition of $\theta_{\mu, \nu, B}$; thus $\bar{M}_{\nu} \leq \bar{M}_{\mu}$. Moreover $\left\{\bar{M}_{\mu}, \bar{\theta}_{\mu, \nu}\right\}$ is a direct system of topological spaces because so is $\left\{\mathcal{E}_{\mu}(M), \theta_{\mu, \nu}\right\}$. Since $\mathcal{E}_{\infty}(M)$ is the injective limit of $\left\{\mathcal{E}_{\mu}(M), \theta_{\mu, \nu}\right\}$, it easily follows that the injective limit of $\left\{\bar{M}_{\mu}, \bar{\theta}_{\mu, \nu}\right\}$ is a compactification $\bar{M}_{\infty}$ of $M$ with corona $\mathcal{E}_{\infty}(M)$.

LEMMA 7.2. Let $X^{\gamma}$ and $X^{\prime \gamma}$ be compactifications of locally compact Hausdorff spaces $X$ and $X^{\prime}$, with coronas $\gamma X$ and $\gamma X^{\prime}$, respectively. Let $\phi: X \rightarrow X^{\prime}$ and $\psi: X^{\prime} \rightarrow X$ be (possibly non-continuous) maps such that:

(i) $\phi$ and $\psi$ have extensions, $\phi^{\gamma}: X^{\gamma} \rightarrow X^{\prime \gamma}$ and $\psi^{\gamma}: X^{\prime \gamma} \rightarrow X^{\gamma}$, which are continuous at the points of $\gamma X$ and $\gamma X^{\prime}$, respectively; and

(ii) $\phi^{\gamma}$ and $\psi^{\gamma}$ restrict to respective homeomorphisms, $\gamma \phi: \gamma X \rightarrow \gamma X^{\prime}$ and $\gamma \psi$ : $\gamma X^{\prime} \rightarrow \gamma X$, which are inverse of each other.

Then there is a bijection $\bar{X} \mapsto \overline{X^{\prime}}$ between the set of compactifications $\bar{X} \leq X^{\gamma}$, with coronas $\partial X$, and the set of compactifications $\overline{X^{\prime}} \leq X^{\prime \gamma}$, with coronas $\partial X^{\prime}$, such that:

(a) $\phi$ and $\psi$ have extensions, $\bar{\phi}: \bar{X} \rightarrow \overline{X^{\prime}}$ and $\bar{\psi}: \overline{X^{\prime}} \rightarrow \bar{X}$, which are continuous at the points of $\partial X$ and $\partial X^{\prime}$, respectively; and

(b) $\bar{\phi}$ and $\bar{\psi}$ restrict to respective homeomorphisms, $\partial \phi: \partial X \rightarrow \partial X^{\prime}$ and $\partial \psi: \partial X^{\prime} \rightarrow$ $\partial X$, which are inverse of each other.

Proof. Take a compactification $\bar{X} \leq X^{\gamma}$, with corona $\partial X$. Thus $\operatorname{id}_{M}$ has a continuous extension $\pi: \gamma X \rightarrow \partial X$, which is an identification. Then the restriction 
$\pi: X^{\gamma} \rightarrow \partial X$ is also an identification. Let $R$ be the equivalence relation on $\gamma X$ whose equivalence classes are the fibers of $\pi$ (thus $\partial X \equiv \gamma X / R$ ), let $R^{\prime}$ be the equivalence relation on $\gamma X^{\prime}$ that corresponds to $R$ via $\gamma \phi$, and let $\partial X^{\prime}=\gamma X^{\prime} / R^{\prime}$. Then $\partial \phi$ induces a homeomorphism $\partial^{\prime} \phi: \partial^{\prime} X \rightarrow \partial^{\prime} Y$. Extend $R^{\prime}$ to $X^{\prime \gamma}$ so that each point of $X^{\prime}$ is only equivalent to itself, and let $\overline{X^{\prime}}=X^{\prime \gamma} / R^{\prime}$. Let $\pi^{\prime}: X^{\prime \gamma} \rightarrow \overline{X^{\prime}}$ be the quotient map, whose restriction $\pi^{\prime}: \gamma X^{\prime} \rightarrow \partial X^{\prime}$ is also the quotient map. Using that $X^{\prime}$ is open in $\overline{X^{\prime}}$, it easily follows that the restriction of $\pi^{\prime}: X^{\prime} \rightarrow \overline{X^{\prime}}$ is an embeddding with dense image. In this way, $\overline{X^{\prime}}$ becomes a compactification of $X^{\prime}$, with corona $\partial X^{\prime}$, satisfying $\overline{X^{\prime}} \leq X^{\prime \gamma}$. This defines the stated mapping $\bar{X} \mapsto \overline{X^{\prime}}$.

The above notation will be kept for the rest of the proof. Extend $R$ to $X^{\gamma}$ so that each point of $X$ is only equivalent to itself. Then, like in the case of $R^{\prime}$, the space $X^{\gamma} / R$ is a compactification of $X$. Moreover the canonical map $X^{\gamma} / R \rightarrow$ $\bar{X}$ is a continuous bijection between compact Hausdorff spaces, and thus it is a homeomorphism, showing that the compactifications $X^{\gamma} / R$ and $\bar{X}$ are equivalent. Since $R$ corresponds to $R^{\prime}$ via $\gamma \psi=(\gamma \phi)^{-1}$, it follows that $\bar{X}$ corresponds to $\overline{X^{\prime}}$ by the mapping defined by $\psi$ in the same way. Hence the mapping defined by $\psi$ is left inverse of the mapping defyned by $\phi$. Reversing also the roles played by $\phi$ and $\psi$, it follows that the mapping $\bar{X} \mapsto \overline{X^{\prime}}$ of the statement is bijective.

Since $\phi^{\gamma}$ is compatible with $R$ and $R^{\prime}$, it induces a map $\bar{\phi}: \bar{X} \rightarrow \overline{X^{\prime}}$. Let us show that $\bar{\phi}$ is continuous at every $\mathbf{e} \in \partial X$. Let $V^{\prime}$ be a neighborhood of $\mathbf{e}^{\prime}:=\bar{\phi}(\mathbf{e})$ in $\overline{X^{\prime}}$. The set $\widetilde{V}^{\prime}:=\pi^{\prime-1}\left(V^{\prime}\right)$ is an $R^{\prime}$-saturated neighborhood of $\pi^{\prime-1}\left(\mathbf{e}^{\prime}\right)$ in $X^{\prime \gamma}$. So, by (i), $\widetilde{V}:=\left(\phi^{\gamma}\right)^{-1}\left(\widetilde{V}^{\prime}\right)=\pi^{-1}\left(\bar{\phi}^{-1}\left(V^{\prime}\right)\right)$ is an $R$-saturated neighborhood of $\widetilde{V}:=$ $\left(\phi^{\gamma}\right)^{-1}\left(\pi^{\prime-1}\left(\mathbf{e}^{\prime}\right)\right)=\pi^{-1}\left(\bar{\phi}^{-1}\left(\mathbf{e}^{\prime}\right)\right)$ in $X^{\gamma}$. Hence $\pi(\widetilde{V})=\bar{\phi}^{-1}\left(V^{\prime}\right)$ is a neighborhood of $\bar{\phi}^{-1}\left(\mathbf{e}^{\prime}\right)$ in $\bar{X}$. Similarly, $\psi$ induces a map $\bar{\psi}: \overline{X^{\prime}} \rightarrow \bar{X}$, which is continuous at the points of $\partial X^{\prime}$. This completes the proof of (a).

Property (b) follows directly from (ii) because $\partial \phi$ and $\partial \psi$ are induced by $\gamma \phi$ and $\gamma \psi$, respectively.

\subsection{Higson compactification}

Suppose that the metric space $M$ is proper. For $R>0$, the $R$-variation a function $f: M \rightarrow \mathbb{C}$ is the function $\vee_{R} f: M \rightarrow \mathbb{C}$ be given by

$$
\mathrm{V}_{R} f(x)=\sup \{|f(x)-f(y)| \mid d(x, y)<R\} .
$$

It is said that $f: M \rightarrow \mathbb{C}$ is a Higson function if it is bounded and $\vee_{R} f$ vanishes at infinity for all $R>0$. The continuos Higson functions on $M$ form a unital closed involutive subalgebra $C_{\nu}(X) \subset C_{b}(X)$ that generates the topology. The compactification of $M$ that corresponds to $C_{\nu}(X)$ is called the Higson compactification, and $\nu M=M^{\nu} \backslash M$ is called the Higson corona of $M$.

REMARK 7.3. (i) The construction of the Higson corona can be extended to the case where $M$ is not proper in the following way. The (possibly non-continuous) Higson functions form a unital closed involutive subalgebra $\mathcal{B}_{\nu}(M)$ of the commutative $C^{*}$ algebra of $\mathbb{C}$-valued bounded functions on $M$ with the supremum norm. Now, it is said that a function $f: M \rightarrow \mathbb{C}$ vanishes at infinity if, for all bounded subset $B \subset M$, there is some $r>0$ such that $|f|<\varepsilon$ on $M \backslash B$. The functions vanishing at infinity form a closed involutive ideal $\mathcal{B}_{0} \subset \mathcal{B}_{\nu}(M)$. Then the Higson corona $\nu M$ is the compact Hausdorff space that corresponds to the unital commutative $C^{*}$ algebra $\mathcal{B}_{\nu}(M) / \mathcal{B}_{0}(M)$; 
this $C^{*}$ algebra is isomorphic to $C_{\nu}(M) / C_{0}(M) \cong C(\nu M)$ when $M$ is proper [86. Lemma 2.40].

(ii) In fact, the Higson compactification can be defined for arbitrary proper coarse spaces, and the Higson corona can be defined for all coarse spaces 86, Section 2.3].

Proposition 7.4 (Álvarez-Candel 11, Corollary 4.14, Proposition 4.15 and Theorem 4.16]). The following properties hold for maps $\phi, \psi: M \rightarrow M^{\prime}$ between proper metric spaces:

(i) $\phi$ is coarse if and only if it has an extension $\phi^{\nu}: M^{\nu} \rightarrow M^{\prime \nu}$ that is continuous at the points of $\nu M$ and such that $\phi^{\nu}(\nu M) \subset \nu M^{\prime}$. In particular, $\phi^{\nu}$ restricts to a continuous map $\nu \phi: \nu M \rightarrow \nu M^{\prime}$.

(ii) $\phi$ is close to $\psi$ if and only if the extensions $\phi^{\nu}$ and $\psi^{\nu}$, given by (i), are equal on $\nu M$.

(iii) $\phi$ is a coarse equivalence if and only if it satisfies the conditions of (i) and $\nu \phi: \nu M \rightarrow \nu M^{\prime}$ is a homeomorphism.

Proposition 7.5. If $\phi: M \rightarrow M^{\prime}$ is rough map, then $\nu \phi: \nu M \rightarrow \nu M^{\prime}$ is an embedding whose image is $\mathrm{Cl}_{M^{\prime \nu}}(\phi(M)) \cap \nu M^{\prime}$.

Proof. By Corollary 2.38 and Proposition 7.4 (iii), it can be assumed that $M$ is a metric subspace of $M^{\prime}$, and $\phi$ is the inclusion map $M \hookrightarrow M^{\prime}$. Since the Higson coronas are compact Hausdorff metric spaces and $\nu \phi$ is continuous (Proposition 7.4. (ii)), it is enough to prove that $\nu \phi$ is injective.

Let $e_{0} \neq e_{1}$ in $\nu M$. Take open subsets $V_{0}, V_{1} \subset M^{\nu}$ such that $e_{i} \in V_{i}(i \in\{0,1\})$ and $\mathrm{Cl}_{M^{\nu}}\left(V_{0}\right) \cap \mathrm{Cl}_{M^{\nu}}\left(V_{1}\right)=\varnothing$. Fix any $x_{0} \in M \backslash \mathrm{Cl}_{M^{\nu}}\left(V_{0} \cup V_{1}\right)$, and let $B_{R}=$ $B_{M}\left(x_{0}, R\right)$ for each $R \geq 0$.

Claim 7. $d\left(V_{0} \backslash B_{R}, V_{1} \backslash B_{R}\right) \rightarrow \infty$ as $R \rightarrow \infty$.

There is a function $F \in C\left(M^{\nu}\right)$ such that $F\left(V_{i}\right)=i$, and let $f=\left.F\right|_{M} \in C_{\nu}(M)$. If Claim 7 were false, there would be some $r>0$ and sequences $x_{i, k} \in V_{i} \backslash B_{k}$ so that $d\left(x_{0, k}, x_{1, k}\right) \leq r$ for all $k$, obtaining the contradiction $1=f\left(x_{0, k}\right)-f\left(x_{1, k}\right) \rightarrow 0$ as $k \rightarrow \infty$ because $f \in C_{\nu}(M)$.

The mapping $R \mapsto d\left(V_{0} \backslash B_{R}, V_{1} \backslash B_{R}\right)$ is non-decreasing and upper semi continuous. It may not be continuous but, using Claim 7 it easily follows that there is a smooth function $\rho:[0, \infty) \rightarrow \mathbb{R}^{+}$such that $\rho(R) \leq d\left(V_{0} \backslash B_{R}, V_{1} \backslash B_{R}\right), 0 \leq \rho^{\prime} \leq 1$, and $\rho(R) \rightarrow \infty$ as $R \rightarrow \infty$; in particular, $\rho(R) \leq \rho(R+r) \leq \rho(R)+r$ for all $R, r \geq 0$.

Now let $f^{\prime}: M^{\prime} \backslash B_{1} \rightarrow \mathbb{C}$ be defined by

$$
f^{\prime}(x)= \begin{cases}\frac{\rho\left(d^{\prime}\left(x, x_{0}\right)\right)-d^{\prime}\left(x, V_{1}\right)}{\rho\left(d^{\prime}\left(x, x_{0}\right)\right)} & \text { if } d^{\prime}\left(x, V_{1}\right) \leq \rho\left(d^{\prime}\left(x, x_{0}\right)\right) \\ 0 & \text { otherwise }\end{cases}
$$

Note that $f^{\prime}$ is continuous, non-negative and bounded, and $f^{\prime}\left(V_{i}\right)=i$. Take some $r>0$ and $x, y \in M^{\prime}$ such that $d^{\prime}(x, y)<r$. For $R=d^{\prime}\left(x, x_{0}\right)$ and $D=d^{\prime}\left(x, V_{1}\right)$, we have $R-r \leq d^{\prime}\left(y, x_{0}\right) \leq R+r$ and $D-r \leq d^{\prime}\left(y, V_{1}\right) \leq D+r$, obtaining

$$
\rho(R)-r \leq \rho(R-r) \leq \rho\left(d^{\prime}\left(y, x_{0}\right)\right) \leq \rho(R+r) \leq \rho(R)+r .
$$


For the sake of simplicity, let $\rho=\rho(R)$ for this particular $R$. If $D+2 r \leq \rho$, we get

$$
\begin{gathered}
f^{\prime}(x)-f^{\prime}(y)=\frac{\rho-D}{\rho}-\frac{\rho-r-(D+r)}{\rho+r}=\frac{\rho-D}{\rho}-\frac{\rho(R)-D+2 r}{\rho+r} \rightarrow 0, \\
f^{\prime}(y)-f^{\prime}(x) \leq \frac{\rho+r-(D-r)}{\rho-r}-\frac{\rho-D}{\rho}=\frac{\rho-D+2 r}{\rho-r}-\frac{\rho-D}{\rho} \rightarrow 0,
\end{gathered}
$$

as $\rho \rightarrow \infty$ (and therefore as $R \rightarrow \infty$ ). If $D+2 r \geq \rho$, we get

$$
\begin{gathered}
f^{\prime}(x)=\frac{\rho-D}{\rho} \leq \frac{2 r}{\rho} \rightarrow 0, \\
f^{\prime}(y) \leq \frac{\rho+r-(D-r)}{\rho-r}=\frac{\rho-D+2 r}{\rho-r} \leq \frac{4 r}{\rho-r} \rightarrow 0,
\end{gathered}
$$

as $\rho \rightarrow \infty$. So $f^{\prime} \in C_{\nu}\left(M^{\prime}\right)$, and therefore $f^{\prime}$ has an extension $F^{\prime} \in C\left(M^{\prime \nu}\right)$. Also, $f:=\left.f^{\prime}\right|_{M} \in C_{\nu}(M)$, whose continuous extension to $M^{\nu}$ is $F:=\left(\phi^{\nu}\right)^{*} F^{\prime}$. Since $f\left(V_{i}\right)=i$, we have $F\left(\mathrm{Cl}_{M^{\nu}}\left(V_{i}\right)\right)=i$, and therefore $i=F\left(e_{i}\right)=F^{\prime}\left(\phi^{\nu}\left(e_{i}\right)\right)=$ $F^{\prime}\left(\nu \phi\left(e_{i}\right)\right)$, obtaining $\nu \phi\left(e_{0}\right) \neq \nu \phi\left(e_{1}\right)$.

COROLlary 7.6. If $\phi: M \rightarrow M^{\prime}$ is a continuous rough map, then $\phi^{\nu}: M^{\nu} \rightarrow$ $M^{\prime \nu}$ is an embedding whose image is $\mathrm{Cl}_{M^{\prime \nu}}(\phi(M))$.

Proof. By Propositions 7.4 (i) and 7.5. $\phi^{\nu}$ is an injective continuous map between compact Hausdorff spaces.

Proposition 7.7 (Álvarez-Candel [11, Proposition 4.12]). If an open subset $W \subset M$ contains balls of arbitrarily large radius, then

$$
\operatorname{Int}_{M^{\nu}}\left(\mathrm{Cl}_{M^{\nu}}(W)\right) \cap \nu M \neq \varnothing \text {. }
$$

Proposition 7.8. For all $W \subset M$ and $r>0$,

$$
\operatorname{Int}_{M^{\nu}}\left(\mathrm{Cl}_{M^{\nu}}(W)\right) \cap \nu M=\operatorname{Int}_{M^{\nu}}\left(\mathrm{Cl}_{M^{\nu}}\left(W_{r}\right)\right) \cap \nu M,
$$

where

$$
W_{r}=\{x \in W \mid d(x, M \backslash W)>r\} .
$$

Proof. The inclusion " $\supset$ " of the statement is obvious.

Let us prove the inclusion " $\subset$ " of the statement. For the sake of simplicity, given $W \subset M$ and $r>0$, let

$$
V=\operatorname{Int}_{M^{\nu}}\left(\mathrm{Cl}_{M^{\nu}}(W)\right), \quad V_{r}=\operatorname{Int}_{M^{\nu}}\left(\mathrm{Cl}_{M^{\nu}}\left(W_{r}\right)\right) .
$$

For any $e \in V \cap \nu M$, there is a continuous function $F: M^{\nu} \rightarrow[0,1]$ such that $F(e)=0$ and $F\left(M^{\nu} \backslash V\right)=1$. Since $f=\left.F\right|_{M} \in C_{\nu}(M)$, there is a compact $K \subset M$ such that $\left|\mathrm{V}_{r+1} f\right|<1 / 2$ on $M \backslash K$.

Claim 8. $f>1 / 2$ on $M \backslash\left(\mathrm{Cl}_{M^{\nu}}\left(W_{r}\right) \cup K\right)$.

For all $u \in M \backslash\left(\mathrm{Cl}_{M^{\nu}}\left(W_{r}\right) \cup K\right)$, there is some $v \in M \backslash W$ so that $d(v, w) \leq$ $r<r+1$. Thus $|f(u)-1|=|f(u)-f(v)|<1 / 2$, obtaining $f(u)>1 / 2$, which shows Claim 8 .

Claim 9. We have

$$
M^{\nu} \backslash\left(\mathrm{Cl}_{M^{\nu}}\left(W_{r}\right) \cup K\right) \subset \mathrm{Cl}_{M^{\nu}}\left(M \backslash\left(\mathrm{Cl}_{M^{\nu}}\left(W_{r}\right) \cup K\right)\right) .
$$


For any open neighborhood $O$ of a point $e^{\prime} \in M^{\nu} \backslash\left(\mathrm{Cl}_{M^{\nu}}\left(W_{r}\right) \cup K\right)$, we get

$$
\varnothing \neq\left(M^{\nu} \backslash\left(\mathrm{Cl}_{M^{\nu}}\left(W_{r}\right) \cup K\right)\right) \cap M \cap O=\left(M \backslash\left(\mathrm{Cl}_{M^{\nu}}\left(W_{r}\right) \cup K\right)\right) \cap O
$$

because $M^{\nu} \backslash\left(\mathrm{Cl}_{M^{\nu}}\left(W_{r}\right) \cup K\right)$ is open in $M^{\nu}$, and $M$ is open and dense in $M^{\nu}$. So $e^{\prime} \in \mathrm{Cl}_{M^{\nu}}\left(M \backslash\left(\mathrm{Cl}_{M^{\nu}}\left(W_{r}\right) \cup K\right)\right)$, showing Claim 9 .

From Claims 8 and 9 , it follows that $F \geq 1 / 2$ on $M^{\nu} \backslash\left(\mathrm{Cl}_{M^{\nu}}\left(W_{r}\right) \cup K\right)$ by the continuity of $F$. Hence

$$
F^{-1}([0,1 / 2)) \subset \operatorname{Int}_{M^{\nu}}\left(\mathrm{Cl}_{M^{\nu}}\left(W_{r}\right) \cup K\right)
$$

because $F^{-1}([0,1 / 2))$ is open in $M^{\nu}$. So

$$
e \in F^{-1}([0,1 / 2)) \cap \nu M \subset \operatorname{Int}_{M^{\nu}}\left(\mathrm{Cl}_{M^{\nu}}\left(W_{r}\right) \cup K\right) \cap \nu M=V_{r} \cap \nu M .
$$

The following corollary states the reciprocal of Proposition 7.7 .

Corollary 7.9. If 7.1 holds for some open subset $W \subset M$, then $W$ contains balls of arbitrarily large radius.

Proof. By Proposition 7.8 , with the notation 7.2 , we get $W_{r} \neq \varnothing$ for all $r>0$. So $B(x, r) \subset W$ for any $x \in W_{r}$.

Corollary 7.10. If 7.1 holds for some open subset $W \subset M$, then

$$
\mathrm{Cl}_{\nu M}\left(\operatorname{Int}_{M^{\nu}}\left(\mathrm{Cl}_{M^{\nu}}(W)\right) \cap \nu M\right)=\mathrm{Cl}_{M^{\nu}}(W) \cap \nu M .
$$

Proof. There is a canonical identity $\nu W \equiv \mathrm{Cl}_{M^{\nu}}(W) \cap \nu M$ by Corollary 7.6. Any open set of $\nu W$ is of the form $V_{0} \cap \nu W$ for some open subset $V_{0} \subset W^{\nu}$. If $V_{0} \cap \nu W \neq \varnothing$, then there is some open subset $V_{1} \subset W^{\nu}$ such that $V_{1} \cap \nu W \neq \varnothing$ and $\mathrm{Cl}_{M^{\nu}}\left(V_{1}\right) \subset V_{0}$. By Corollary 7.9. $V_{1} \cap W$ contains balls of arbitrarily large radii by Corollary 7.9 , So

$$
\varnothing \neq \operatorname{Int}_{M^{\nu}}\left(\mathrm{Cl}_{M^{\nu}}\left(V_{1} \cap W\right)\right) \cap \nu M \subset \operatorname{Int}_{M^{\nu}}\left(\mathrm{Cl}_{M^{\nu}}(W)\right) \cap \nu M
$$

by Proposition 7.7. On the other hand,

$$
\operatorname{Int}_{M^{\nu}}\left(\mathrm{Cl}_{M^{\nu}}\left(V_{1} \cap W\right)\right) \cap \nu M \subset V_{0} \cap \mathrm{Cl}_{M^{\nu}}(W) \cap \nu M=V_{0} \cap \nu W .
$$

Hence $V_{0} \cap \nu W$ meets $\operatorname{Int}_{M^{\nu}}\left(\mathrm{Cl}_{M^{\nu}}(W)\right) \cap \nu M$.

Proposition 7.11. Let $\phi: M \rightarrow M^{\prime}$ be an $\left(s_{r}, c\right)$-rough equivalence. Given a compactification $\bar{M} \leq M^{\nu}$, according to Lemma $\overline{\text { 7.2. }} \overline{M^{\prime}} \leq M^{\prime \nu}$ be the corresponding compactification, and let $\bar{\phi}: \bar{X} \rightarrow \overline{X^{\prime}}$ be the map induced by $\phi$, whose restriction to the coronas is denoted by $\partial \phi: \partial M \rightarrow \partial M^{\prime}$. Let $\mathbf{e} \in \partial M$, and let $\mathcal{V}$ be a base of neighborhoods of e in $\bar{M}$. Then

$$
\mathcal{V}^{\prime}=\left\{\mathrm{Cl}_{\overline{M^{\prime}}}\left(\operatorname{Pen}_{M^{\prime}}(\phi(V \cap M), c)\right) \mid V \in \mathcal{V}\right\}
$$

is a base of neighborhoods of $\partial \phi(e)$ in $\overline{M^{\prime}}$.

Proof. Let us consider first the case where $\bar{M}=M^{\nu}$, and thus $\overline{M^{\prime}}=M^{\prime \nu}$, $\bar{\phi}=\phi^{\nu}$ and $\partial \phi=\nu \phi$.

Let us prove that any element $\mathrm{Cl}_{M^{\prime \nu}}\left(\operatorname{Pen}_{M^{\prime}}(\phi(V), c)\right)$ of $\mathcal{V}^{\prime}$, defined by some $V \in \mathcal{V}$, is a neighborhood of $e^{\prime}:=\nu \phi(e)$. Let $\psi: M^{\prime} \rightarrow M$ be another $s_{r}$-rough equivalence such that $\psi \phi$ and $\phi \psi$ are $c$-close to $\mathrm{id}_{M}$ and $\operatorname{id}_{M^{\prime}}$, respectively. By Proposition 7.4-(i), there is some open neighborhood $V^{\prime}$ of $e^{\prime}$ in $M^{\prime \nu}$ such that 
$\psi^{\nu}\left(V^{\prime}\right) \subset V$. For any $x \in V^{\prime} \cap M^{\prime}$, we have $d^{\prime}(x, \phi \psi(x)) \leq c$ and $\psi(x) \in V$. So $V^{\prime} \cap M^{\prime} \subset \operatorname{Pen}_{M^{\prime}}(\phi(V), c)$, giving

$$
\mathrm{Cl}_{M^{\prime \nu}}\left(V^{\prime} \cap M^{\prime}\right) \subset \mathrm{Cl}_{M^{\prime \nu}}\left(\operatorname{Pen}_{M^{\prime}}(\phi(V), c)\right) .
$$

But $V^{\prime} \subset \mathrm{Cl}_{M^{\prime \nu}}\left(V^{\prime} \cap M^{\prime}\right)$ because $V^{\prime}$ is open in $M^{\prime \nu}$ and $M^{\prime}$ is open and dense in $M^{\prime \nu}$. So $\mathrm{Cl}_{M^{\prime \nu}}\left(\operatorname{Pen}_{M^{\prime}}(\phi(V), c)\right)$ is an open neighborhood of $e^{\prime}$.

Now, let us prove that any neighborhood $V^{\prime}$ of $e^{\prime}$ in $M^{\prime \nu}$ contains some element of $\mathcal{V}^{\prime}$. Take another neighborhood $V_{0}^{\prime}$ of $e^{\prime}$ in $M^{\prime \nu}$ with $\mathrm{Cl}_{M^{\prime \nu}}\left(V_{0}^{\prime}\right) \subset V^{\prime}$. Let $W^{\prime}=V_{0} \cap M^{\prime}$ and, for any $r>c$, let $W_{r}^{\prime}$ be defined by (7.2). By (2.3) and since $\operatorname{Pen}_{M^{\prime}}\left(W_{r}^{\prime}, r\right) \subset W^{\prime}$, we get $\operatorname{Pen}_{M^{\prime}}\left(\mathrm{Cl}_{M^{\prime}}\left(W_{r}^{\prime}\right), c\right) \subset W^{\prime}$. By Proposition 7.8, the set $V_{0, r}^{\prime}:=\operatorname{Int}_{M^{\prime \nu}}\left(\mathrm{Cl}_{M^{\prime \nu}}\left(W_{r}^{\prime}\right)\right)$ is another neighborhood of $e^{\prime}$ in $M^{\prime \nu}$. By Proposition 7.4.1i), there is some $V \in \mathcal{V}$ such that $\mathrm{Cl}_{M^{\prime \nu}}\left(\phi^{\nu}(V)\right) \subset V_{0, r}^{\prime}$. Hence

$$
\begin{aligned}
\mathrm{Cl}_{M^{\prime \nu}}\left(\operatorname{Pen}_{M^{\prime}}\right. & (\phi(V \cap M), c)) \subset \mathrm{Cl}_{M^{\prime \nu}}\left(\operatorname{Pen}_{M^{\prime}}\left(V_{0, r}^{\prime} \cap M^{\prime}, c\right)\right) \\
& \subset \mathrm{Cl}_{M^{\prime \nu}}\left(\operatorname{Pen}_{M^{\prime}}\left(\mathrm{Cl}_{M^{\prime}}\left(W_{r}^{\prime}\right), c\right)\right) \subset \mathrm{Cl}_{M^{\prime \nu}}\left(W^{\prime}\right) \subset \mathrm{Cl}_{M^{\prime \nu}}\left(V_{0}^{\prime}\right) \subset V^{\prime} .
\end{aligned}
$$

This completes the proof in the case $\bar{M}=M^{\nu}$.

Now consider the general case. Let $\pi: M^{\nu} \rightarrow \bar{M}$ and $\pi^{\prime}: M^{\prime \nu} \rightarrow \overline{M^{\prime}}$ be continuous extensions of $\operatorname{id}_{M}$ and $\operatorname{id}_{M^{\prime}}$, respectively. Given $e$ and $\mathcal{V}$ like in the statement, the sets $\widetilde{V}:=\pi^{-1}(V)$, for $V \in \mathcal{V}$, form a base $\widetilde{\mathcal{V}}$ of neighborhoods of any $\widetilde{e} \in \pi^{-1}(e)$ in $M^{\nu}$. So, by the above case, it is easy to see that the sets $\widetilde{V}^{\prime}:=\mathrm{Cl}_{M^{\prime \nu}}\left(\operatorname{Pen}_{M^{\prime}}(\phi(\widetilde{V} \cap M), c)\right)$, for $\widetilde{V} \in \widetilde{\mathcal{V}}$, form a base of neighborhoods of $\nu \phi\left(\pi^{-1}(e)\right)=\pi^{\prime-1}(\partial \phi(e))$ in $M^{\nu}$. Since the sets $\widetilde{V}^{\prime}$ are saturated by the fibers of $\pi^{\prime}$, it follows that the sets $\pi^{\prime}\left(\widetilde{V}^{\prime}\right)=\mathrm{Cl}_{\overline{M^{\prime}}}\left(\operatorname{Pen}_{M^{\prime}}(\phi(V \cap M), c)\right)$, for $V \in \mathcal{V}$, form a base of neighborhoods of $\partial \phi(e)$.

\subsection{Asymptotic dimension}

Let $\mathcal{V}$ be a cover of a space $X$. The multiplicity of a $\mathcal{V}$ is the least $n \in \mathbb{N} \cup\{\infty\}$ such that there are at most $n$ elements of $\mathcal{V}$ meeting at any point of $X$. It is said that $\mathcal{V}$ refines another cover $\mathcal{W}$ of $X$ if every element of $\mathcal{V}$ is contained in some element of $\mathcal{W}$. Recall that the Lebesgue covering dimension of $X$ is the least $n \in \mathbb{N} \cup\{\infty\}$ such that every open cover of $X$ is refined by a cover with multiplicity $\leq n+1$

A family $\mathcal{V}$ of subsets of the metric space $M$ is called uniformly bounded if there is some $D>0$ such that $\operatorname{diam} V \leq D$ for all $V \in \mathcal{V}$. The following definition is somehow dual to the definition of Lebesgue covering dimension.

Definition 7.12. The asymptotic dimension of $M$, denoted asdim $M$, is the least $n \in \mathbb{N} \cup\{\infty\}$ such that any uniformly bounded open cover of $X$ refines some uniformly bounded open cover with multiplicity $\leq n+1$.

REMARK 7.13. The asymptotic dimension was introduced by Gromov [55] in a different way. We follow the survey [16] by Bell and Dranishnikov, which contains many relevant examples and results about the asymptotic dimension.

Proposition 7.14 (See e.g. 16, Theorem 1]). For $n \in \mathbb{N}$, we have asdim $M \leq n$ if and only if, for any $R>0$, there exist uniformly bounded families $\mathcal{V}_{0}, \ldots, \mathcal{V}_{n}$ of subsets of $M$ such that $\bigcup_{i=0}^{n} \mathcal{V}_{i}$ is a cover of $M$, and $d\left(V, V^{\prime}\right)>R$ for $V \neq V^{\prime}$ in any $\mathcal{U}_{i}$.

${ }^{4}$ The original notation of Gromov [55 is as $\operatorname{dim}_{+} M$. 
Proposition 7.15 (See e.g. [16, Proposition 2]). Corsely equivalent metric spaces have the same asymptotic dimension.

EXAMPLES 7.16. We have $\operatorname{asdim} T \leq 1$ for any tree $T\left[\mathbf{1 6}\right.$, asdim $\mathbb{H}^{n}=n$ for the hyperbolic space $\mathbb{H}^{n}\left[\mathbf{5 5}\right.$, and asdim $\mathbb{R}^{n}=n$ for Euclidean space $\mathbb{R}^{n}[\mathbf{3 8}$, [16]. For any finitely generated group $\Gamma$, we have $\operatorname{asdim} \Gamma=0$ if and only if $\Gamma$ is finite [16]. 


\section{CHAPTER 8}

\section{Pseudogroups}

This chapter mainly recalls basic notions and results on pseudogroups, and fixes the notation. Most of these preliminaries can be seen in [57, [58, [59] and [10. Some new results are also proved.

\subsection{Pseudogroups}

A collection, $\mathcal{H}$, of homeomorphisms between open subsets of a topological space $Z$ is called a pseudogroup of local transformations of $Z$ (or simply a pseudogroup on $Z$ ) if $\operatorname{id}_{Z} \in \mathcal{H}$, and $\mathcal{H}$ is closed under composition (wherever defined ${ }^{1}$ ), inversion, restriction (to open subsets) and combination (or union) of maps. A subset $E \subset \mathcal{H}$ of the pseudogroup $\mathcal{H}$ is called symmetric when $h^{-1} \in E$ for all $h \in E$, and the pseudogroup $\mathcal{H}$ is said to be generated by $E$ if every element of $\mathcal{H}$ can be obtained from $E$ by using the above pseudogroup operations. The restriction of $\mathcal{H}$ to an open subset $U$ of $Z$ is the pseudogroup on $U$ given by

$$
\left.\mathcal{H}\right|_{U}=\{h \in \mathcal{H} \mid \operatorname{dom} h \cup \operatorname{im} h \subset U\} .
$$

Let $\mathcal{H}^{\prime}$ be another pseudogroup on a space $Z^{\prime}$. Then $\mathcal{H} \times \mathcal{H}^{\prime}$ denotes the pseudogroup on $Z \times Z^{\prime}$ generated by the maps $h \times h^{\prime}$ with $h \in \mathcal{H}$ and $h^{\prime} \in \mathcal{H}^{\prime}$.

A pseudogroup on a space is an obvious generalization of a group acting on a space via homeomorphisms, and so all basic concepts from the theory of group actions can be generalized to pseudogroups. For instance, the orbit (or $\mathcal{H}$-orbit, or trajectory) of each $x \in Z$ is the set

$$
\mathcal{H}(x)=\{h(x) \mid h \in \mathcal{H}, x \in \operatorname{dom} h\} .
$$

The orbits of $\mathcal{H}$ form a partition of $Z$. The corresponding quotient space (the orbit space) is denoted by $Z / \mathcal{H}$.

Definition 8.1 (Haefliger [57, [58). An étale morphism $\Phi: \mathcal{H} \rightarrow \mathcal{H}^{\prime}$ is a maximal set of homeomorphisms of open subsets of $Z$ to open subsets of $Z^{\prime}$ such that:

- if $\phi \in \Phi, h \in \mathcal{H}$ and $h^{\prime} \in \mathcal{H}^{\prime}$, then $h^{\prime} \phi h \in \Phi$;

- the sources of elements of $\Phi$ cover $Z$; and,

- if $\phi, \psi \in \Phi$, then $\psi \phi^{-1} \in \mathcal{H}^{\prime}$.

An étale morphism $\Phi: \mathcal{H} \rightarrow \mathcal{H}^{\prime}$ is called an equivalence if $\Phi^{-1}=\left\{\phi^{-1} \mid \phi \in \Phi\right\}$ is also an étale morphism $\mathcal{H}^{\prime} \rightarrow \mathcal{H}$, which is called the inverse of $\Phi$. An étale morphism $\Phi: \mathcal{H} \rightarrow \mathcal{H}^{\prime}$ is generated by a subset $\Phi_{0} \subset \Phi$ if all the elements of $\Phi$ can be obtained by combination of composites $h^{\prime} \phi h$ with $h \in \mathcal{H}, \phi \in \Phi_{0}$ and $h^{\prime} \in \mathcal{H}^{\prime}$. The composite of two étale morphisms, $\Phi: \mathcal{H} \rightarrow \mathcal{H}^{\prime}$ and $\Psi: \mathcal{H}^{\prime} \rightarrow \mathcal{H}^{\prime \prime}$, is the étale morphism $\Psi \Phi: \mathcal{H} \rightarrow \mathcal{H}^{\prime \prime}$ generated by $\{\psi \phi \mid \phi \in \Phi, \psi \in \Psi\}$.

\footnotetext{
${ }^{1}$ Composite of partial maps
} 
An étale morphism $\Phi: \mathcal{H} \rightarrow \mathcal{H}^{\prime}$ clearly induces a continuous map $\bar{\Phi}: Z / \mathcal{H} \rightarrow$ $Z^{\prime} / \mathcal{H}^{\prime}$, which is a homeomorphism if $\Phi$ is an equivalence. If $\mathcal{H}$ and $\mathcal{H}^{\prime}$ are equivalent, then they should be considered as the same generalized dynamical system. Thus the properties of pseudogroups that are invariant by equivalences are especially relevant.

EXAmple 8.2. If $U \subset Z$ is an open subset that meets every $\mathcal{H}$-orbit, then the inclusion map $U \hookrightarrow Z$ generates an equivalence $\left.\mathcal{H}\right|_{U} \rightarrow \mathcal{H}$.

REMARK 8.3. Example 8.2 can be used to describe any pseudogroup equivalence $\Phi: \mathcal{H} \rightarrow \mathcal{H}^{\prime}$ as follows. Let $\mathcal{H}^{\prime \prime}$ be the pseudogroup on $Z^{\prime \prime}=Z \sqcup Z^{\prime}$ generated by $\mathcal{H} \cup \mathcal{H}^{\prime} \cup \Phi$, and let $\Psi: \mathcal{H} \rightarrow \mathcal{H}^{\prime \prime}$ and $\Psi^{\prime}: \mathcal{H}^{\prime} \rightarrow \mathcal{H}^{\prime \prime}$ be the equivalences generated by $Z \hookrightarrow Z^{\prime \prime}$ and $Z^{\prime} \leftrightarrow Z^{\prime \prime}$, respectively. Then $\Phi=\Psi^{\prime-1} \Psi$.

The germ groupoid of $\mathcal{H}$ is the topological groupoid of germs of maps in $\mathcal{H}$ at all points of their domains, with the operation induced by the composite of partial maps and the étale topology. Its subspace of units can be canonically identified with $Z$. For each $x \in Z$, the group of elements of this groupoid whose source and range is $x$ will be called the germ group of $\mathcal{H}$ at $x$. The germ groups at points in the same orbit are isomorphic by conjugation in the germ groupoid. Thus the germ group of each orbit is defined up to isomorphisms. By Remark 8.3 , it follows that, under pseudogroup equivalences, corresponding orbits have isomorphic germ groups.

Let $\mathcal{H}$ be a pseudogroup on a locally compact space $Z$. Then the orbit space $Z / \mathcal{H}$ is compact if and only if $Z$ has a relatively compact open subset that meets every $\mathcal{H}$-orbit. The following is a stronger compactness condition on $\mathcal{H}$.

Definition 8.4 (Haefliger [57]). A pseudogroup, $\mathcal{H}$, is compactly generated if there is a relatively compact open set $U$ in $Z$, meeting each orbit, such that $\left.\mathcal{H}\right|_{U}$ has a finite symmetric set of generators, $E$, so that each $g \in E$ has an extension $\bar{g} \in \mathcal{H}$ with $\overline{\operatorname{dom} g} \subset \operatorname{dom} \bar{g}$. In this case, $E$ is called a system of compact generation of $\mathcal{H}$ on $U$.

It was observed in $\mathbf{5 7}$ that this notion is invariant by equivalences, and that the relatively compact open set $U$ meeting each orbit can be chosen arbitrarily.

\subsection{Coarse quasi-isometry type of orbits}

Let $\mathcal{H}$ be a pseudogroup on a space $Z$, and let $E$ be a symmetric set of generators of $\mathcal{H}$. Each $\mathcal{H}$-orbit $\mathcal{O}$ is the set of vertices of a connected graph, defined by attaching an edge to vertices $x, y \in \mathcal{O}$ whenever $y=h(x)$ for some $h \in E$ with

$x \in \operatorname{dom} h$. This connected graph structure induces a metric $d_{E}$ on $\mathcal{O}$ according to Section 3.1. For $x \in \mathcal{O}, S \subset \mathcal{O}$ and $r \geq 0$, the open and closed $r$-balls of center $x$ in $\left(\mathcal{O}, d_{E}\right)$ are denoted by $B_{E}(x, r)$ and $\bar{B}_{E}(x, r)$, and the $r$-penumbra of $S$ is denoted by $\operatorname{Pen}_{E}(S, r)$.

We focus on the following case. Suppose that $Z$ is locally compact and $\mathcal{H}$ is compactly generated. Let $U \subset Z$ be a relatively compact open subset that meets all orbits, let $E$ be a symmetric system of compact generation of $\mathcal{H}$ on $U$, and let $\mathcal{G}=\left.\mathcal{H}\right|_{U}$. Then we consider the metric $d_{E}$ on the $\mathcal{G}$-orbits. Even under these conditions, the coarse quasi-isometry type of the $\mathcal{G}$-orbits may depend on the choice of $E$ [10, Section 6]. In [10, this problem is solved by introducing the following additional condition. 
Definition 8.5 (Álvarez-Candel [10, Definition 4.2]). E is called recurrent if there exists a relatively compact open subset $V \subset U$ whose intersections with all $\mathcal{G}$-orbits are equi-nets in the $\mathcal{G}$-orbits with $d_{E}$.

According to the following result, the role played by $V$ in Definition 8.5 can actually be played by any relatively compact open subset of $U$ that meets all $\mathcal{G}$ orbits.

Proposition 8.6 (Álvarez-Candel [10, Lemma 4.3]). If $E$ is recurrent and $W \subset U$ is an open subset that meets every $\mathcal{G}$-orbit, then the intersections of all $\mathcal{G}$-orbits with $W$ are equi-nets in the $\mathcal{G}$-orbits with $d_{E}$.

The following result guarantees the existence of recurrent systems of compact generation.

Proposition 8.7 (Álvarez-Candel [10, Corollary 4.5]). There exists a recurrent system $E$ of compact generation of $\mathcal{H}$ on $U$ such that the extension $\bar{g} \in \mathcal{H}$ of each $g \in E$ with $\overline{\operatorname{dom} g} \subset \operatorname{dom} \bar{g}$ can be chosen so that $\bar{E}=\{\bar{g} \mid g \in E\}$ is also a recurrent symmetric system of compact generation on some relatively compact open subset $U^{\prime} \subset Z$ containing $\bar{U}$.

Let $\mathcal{H}^{\prime}$ be another compactly generated pseudogroup on a locally compact space $Z^{\prime}$, let $U^{\prime}$ be a relatively compact open subset of $Z^{\prime}$ that meets all $\mathcal{H}^{\prime}$-orbits, let $E^{\prime}$ be recurrent symmetric system of compact generation for $\mathcal{H}^{\prime}$ on $U^{\prime}$, and let $\mathcal{G}^{\prime}=\left.\mathcal{H}^{\prime}\right|_{U^{\prime}}$

Theorem 8.8 (Álvarez-Candel [10, Theorem 4.6]). With the above notation, suppose that there exists an equivalence $\mathcal{H} \rightarrow \mathcal{H}^{\prime}$, and consider the induced equivalence $\mathcal{G} \rightarrow \mathcal{G}^{\prime}$ and homeomorphism $U / \mathcal{G} \rightarrow U^{\prime} / \mathcal{G}^{\prime}$. Then the $\mathcal{G}$-orbits, endowed with $d_{E}$, are equi-coarsely quasi-isometric to the corresponding $\mathcal{G}^{\prime}$-orbits, endowed with $d_{E^{\prime}}$.

Theorem 8.8 implies the invariance of the coarse quasi-isometry type of the orbits by equivalences when appropriate representatives of pseudogroups and generators are chosen. The following result gives a more explicit relation between $d_{E}$ and $d_{E^{\prime}}$ in a particular case.

Proposition 8.9. Suppose that $Z=Z^{\prime}, \mathcal{H}=\mathcal{H}^{\prime}$ and $U \subset U^{\prime}$. Then $d_{E}$ and the restrictions of $d_{E^{\prime}}$ to the $\mathcal{G}$-orbits are equi-Lipschitz equivalent.

Proof. If $U=U^{\prime}$, this was established in [10, Corollary 4.9]. Assume, by enlarging $E$ if necessary, that $E \subset E^{\prime}$. Then $d_{E^{\prime}}(x, y) \leq d_{E}(x, y)$ for all $x, y \in U$ in the same $\mathcal{G}$-orbit.

On the other hand, by Proposition 8.6 , there is some $R \in \mathbb{N}$ such that $\mathcal{O}^{\prime} \cap U$ is an $R$-net in $\left(\mathcal{O}^{\prime}, d_{E^{\prime}}\right)$ for every $\mathcal{G}^{\prime}$-orbit $\mathcal{O}^{\prime}$. Let $F$ be the family composites, $f$, of at most $R$ maps in $E^{\prime}$ such that $\operatorname{im} f \subset U$. Then $U^{\prime}=\bigcup_{f \in F} \operatorname{dom} f$. Let

$$
G=\left\{f_{2}^{-1} g^{\prime} f_{1} \mid f_{1}, f_{2} \in F \cup\left\{\operatorname{id}_{U}\right\}, g^{\prime} \in E^{\prime}\right\} .
$$

Then $G$ is symmetric because $E^{\prime}$ is symmetric. Moreover every $g \in G$ has an extension $\bar{g} \in \mathcal{H}$ with $\overline{\operatorname{dom} g} \subset \operatorname{dom} \bar{g}$ because the elements of $E^{\prime}$ have such type of extensions. It follows from the definition of $G$ that $E \subset G$ because $E \subset E^{\prime}$, and therefore $G$ is a recurrent symmetric system of compact generation of $\mathcal{H}$ on $U$. By 10. Corollary 4.9] (the case $U=U^{\prime}$ ), there is $C \geq 1$ such that $d_{E}(x, y) \leq C d_{G}(x, y)$ 
for every $x, y \in U$ in the same $\mathcal{G}$-orbit. If $x \neq y$ are in the same $\mathcal{G}$-orbit and have $d_{E^{\prime}}(x, y)=m \geq 1$, then there are $g_{1}^{\prime}, \ldots, g_{m}^{\prime} \in E^{\prime}$ such that $y=g_{m}^{\prime} \cdots g_{1}^{\prime}(x)$, and $f_{0}, \ldots, f_{m} \in F$ such that $f_{1}=f_{m}=\operatorname{id}_{U}$ and $g_{k}^{\prime} \cdots g_{1}^{\prime}(x) \in \operatorname{dom} f_{k}$ for all $k \epsilon$ $\{1, \ldots, n-1\}$. Thus $g_{k}=f_{k} g_{k}^{\prime} f_{k-1} \in G$ for all $k \in\{1, \ldots, m\}$ and $y=g_{m} \cdots g_{1}(x)$, obtaining $d_{G}(x, y) \leq m$. So $d_{E}(x, y) \leq C d_{E^{\prime}}(x, y)$.

REMARK 8.10. In the case $U=U^{\prime}$, without assuming that $E^{\prime}$ is recurrent, [10. Corollary 4.9] in fact states that $E^{\prime}$ is recurrent if and only if $d_{E}$ and $d_{E^{\prime}}$ are equi-Lipschitz equivalent on the $\mathcal{G}$-orbits.

\subsection{A version of local Reeb stability}

Let $\mathcal{H}$ be a compactly generated pseudogroup on a locally compact space $Z$, let $U$ be a relatively compact open subset of $Z$ that meets all $\mathcal{H}$-orbits, let $E$ be recurrent symmetric system of compact generation of $\mathcal{H}$ on $U$, and let $\mathcal{G}=\left.\mathcal{H}\right|_{U}$.

The following notation will be used. For each $m \in \mathbb{Z}^{+}$, let $E^{m}$ denote the $m$-fold Cartesian product $E \times \cdots \times E$, and set $E^{0}=\left\{\operatorname{id}_{Z}\right\}$. For every $g=\left(g_{1}, \ldots, g_{m}\right) \in E^{m}$, its domain is the set $\operatorname{dom} g=\operatorname{dom}\left(g_{1} \cdots g_{m}\right)$, which may be empty. Moreover let $g(x)=g_{1} \cdots g_{m}(x)$ for every $x \in \operatorname{dom} g$, and let $g^{-1}=\left(g_{m}^{-1}, \ldots, g_{1}^{-1}\right)$. For another $n \in \mathbb{Z}^{+}$and $h=\left(h_{1}, \ldots, h_{n}\right) \in E^{n}$, let $g h=\left(g_{1}, \ldots, g_{m}, h_{1}, \ldots, h_{n}\right) \in E^{m+n}$. Finally, for $r \in \mathbb{Z}^{+}$, let $E^{\leq r}=\bigcup_{m=1}^{r} E^{m}$.

By Proposition 8.7, each $g \in E$ has an extension $\bar{g}$ such that $\overline{\operatorname{dom} g} \subset \operatorname{dom} \bar{g}$, and the collection $\bar{E}=\{\bar{g} \mid g \in E\}$ is a recurrent symmetric system of compact generation on some relatively compact open subset $U^{\prime} \subset Z$ with $\bar{U} \subset U^{\prime}$. Let $\mathcal{G}^{\prime}=\left.\mathcal{H}\right|_{U^{\prime}}$. For $m \in \mathbb{Z}^{+}$and $g=\left(g_{1}, \ldots, g_{m}\right) \in E^{m}$, let $\bar{g}=\left(\bar{g}_{1}, \ldots, \bar{g}_{m}\right) \in \bar{E}^{m}$. There is some $C \in \mathbb{Z}^{+}$such that, for all $x, y \in U$ in the same $\mathcal{G}$-orbit,

$$
d_{\bar{E}}(x, y) \leq d_{E}(x, y) \leq C d_{\bar{E}}(x, y) .
$$

In 8.1 above, the first inequality holds because $\bar{g}$ is an extension of the corresponding $g \in E$, and the second inequality follows from Proposition 8.9. On the other hand, by Proposition 8.6, there is some $R \in \mathbb{N}$ so that $\mathcal{O}^{\prime} \cap U$ is an $R$-net in $\left(\mathcal{O}^{\prime}, d_{\bar{E}}\right)$ for every $\mathcal{G}^{\prime}$-orbit $\mathcal{O}^{\prime}$.

Let $U_{0}$ be the set of points in $U$ where $\mathcal{G}$ has trivial germ groups. The following is a coarsely quasi-isometric pseudogroup version of the Reeb local stability around points in $U_{0}$, which will play a very important role in the present work.

Proposition 8.11. For every $r \in \mathbb{Z}^{+}$and $x \in U_{0}$, there exists an open neighborhood $V(x, r)$ of $x$ in $U$ such that:

(i) $V\left(x, r^{\prime}\right) \subset V(x, r)$ if $r^{\prime}>r$;

(ii) $V(x, r) \subset \operatorname{dom} g$ for all $g \in E^{\leq r}$ with $x \in \operatorname{dom} g$;

(iii) for every $y \in V(x, r)$, a map $\phi_{x, y, r}: \bar{B}_{E}(x, r) \rightarrow \bar{B}_{E}(y, r)$ is determined by the condition $\phi_{x, y, r} g(x)=g(y)$ for all $g \in E^{\leq r}$ with $x \in \operatorname{dom} g$;

(iv) $\phi_{x, y, r}$ is non-expanding with respect to $d_{E}$;

(v) $\phi_{x, y, r}$ is $C$-bi-Lipschitz with respect to $d_{E}$; and,

(vi) if $r \geq C R$, then $\bar{B}_{E}(y, r / C) \cap \operatorname{im} \phi_{x, y, r}$ is a $2 C R$-net in $\left(\bar{B}_{E}(y, r / C), d_{E}\right)$.

Proof. Since all the sets $E^{m}$ and $\bar{E}^{m}$ are finite, and since $\overline{\operatorname{dom} g} \subset \operatorname{dom} \bar{g}$ for each $g \in E^{m}$, there is, for every $r \in \mathbb{Z}^{+}$and $x \in U_{0}$, a largest open neighborhood $V(x, r)$ of $x$ in $U$ satisfying (ii) and the following properties: 
(a) if $g(x)=x$ for some $g \in E^{\leq 4 r}$ and $x \in \operatorname{dom} g$, then $V(x, r) \subset \operatorname{dom} g$ and $g(y)=y$ for all $y \in V(x, r)$;

(b) if $V(x, r)$ meets the domain of some $g \in E^{\leq 4 r}$, then $x \in \operatorname{dom} \bar{g}$;

(c) if $g(y)=y$ for some $g \in E^{\leq 4 r}$ and $y \in V(x, r) \cap \operatorname{dom} g$, then $\bar{g}(x)=x$;

(d) $V(x, r) \subset \operatorname{dom} \bar{g}$ for all $\bar{g} \in \bar{E}^{\leq r}$ with $x \in \operatorname{dom} \bar{g}$; and,

(e) if $x \in \operatorname{dom} \bar{g}$ and $\bar{g}(x)=x$ for some $\bar{g} \in \bar{E}^{\leq 4 r}$, then $V(x, r) \subset \operatorname{dom} \bar{g}$ and $\bar{g}(y)=y$ for all $y \in V(x, r)$.

We also get (ii) since $V(x, r)$ is maximal.

Fix $x \in U_{0}, r \in \mathbb{Z}^{+}$and $y \in V(x, r)$. Each point in $\bar{B}_{E}(x, r)$ is of the form $g(x)$ for some $g \in E^{\leq r}$ with $x \in \operatorname{dom} g$, and thus, by (iii), $y \in \operatorname{dom} g$. Suppose that $g(x)=h(x)$ for another $h \in E^{\leq r}$ with $x \in \operatorname{dom} h$. Then $h^{-1} g \in E^{\leq 2 r}, x \in \operatorname{dom} h^{-1} g$ and $h^{-1} g(x)=x$. By (a), it follows that $y \in \operatorname{dom} h^{-1} g$ and $h^{-1} g(y)=y$; i.e., $g(y)=h(y)$. Therefore the assignment $g(x) \mapsto g(y)$ for $g \in E^{\leq r}$ defines a map $\phi_{x, y, r}: \bar{B}_{E}(x, r) \rightarrow \bar{B}_{E}(y, r)$, which is the statement of (iii). For the sake of simplicity, $\phi_{x, y, r}$ will be simply denoted by $\phi$ in the rest of the proof.

For every $z, z^{\prime} \in \bar{B}_{E}(x, r)$ there are $g, g^{\prime} \in E^{\leq r}$ whose domains contain $x$ and such that $g(x)=z$ and $g^{\prime}(x)=z^{\prime}$. If $m=d_{E}\left(z, z^{\prime}\right) \leq 2 r$, there is $h \in E^{m}$ so that $z \in \operatorname{dom} h$ and $h\left(z^{\prime}\right)=z$. Thus $x \in \operatorname{dom} g^{-1} h g^{\prime}$ and $g^{-1} h g^{\prime}(x)=x$ with $g^{-1} h g^{\prime} \in E^{\leq 4 r}$. It follows from (a) that $y \in \operatorname{dom} g^{-1} h g^{\prime}$ and $g^{-1} h g^{\prime}(y)=y$. Hence $g^{\prime}(y) \in \operatorname{dom} h$ and $h g^{\prime}(y)=g(y)$, giving $d_{E}\left(g(y), g^{\prime}(y)\right) \leq m$, which shows (iv).

Let $z, z^{\prime} \in \bar{B}_{E}(x, r)$, and let $g, g^{\prime} \in E^{\leq r}$ be such that $z=g(x)$ and $z^{\prime}=g\left(x^{\prime}\right)$. Thus $y \in \operatorname{dom} g \cap \operatorname{dom} g^{\prime}, \phi(z)=g(y)$ and $\phi\left(z^{\prime}\right)=g^{\prime}(y)$. If $m=d_{E}\left(g(y), g^{\prime}(y)\right) \leq 2 r$, then there is $h \in E^{m}$ so that $g^{\prime}(y) \in \operatorname{dom} h$ and $h g^{\prime}(y)=g(y)$. Hence $g^{-1} h g^{\prime} \in E^{\leq 4 r}$, $y \in \operatorname{dom} g^{-1} h g^{\prime}$ and $g^{-1} h g^{\prime}(y)=y$. By (b) and (c), it follows that $x \in \operatorname{dom} \bar{g}^{-1} \bar{h} \bar{g}^{\prime}$ and $\bar{g}^{-1} \bar{h} \bar{g}^{\prime}(x)=x$, and thus $\bar{h}(z)=z^{\prime}$ with $\bar{h} \in \bar{E}^{m}$. Therefore

$$
d_{E}\left(z, z^{\prime}\right) \leq C d_{\bar{E}}\left(z, z^{\prime}\right) \leq C d_{E}\left(\phi(z), \phi\left(z^{\prime}\right)\right),
$$

by (8.1). This shows $\mathrm{V}$ (considering (iv)).

Observe that (b) and (iv) only require (iii) and (a). Thus, by (d) and (e), using $\bar{E}$ instead of $E$, for each $y \in V(x, r)$ there is a map $\bar{\phi}_{x, y, r}: \bar{B}_{\bar{E}}(x, r) \rightarrow \bar{B}_{\bar{E}}(y, r)$, which is determined by the condition that $\bar{\phi}_{x, y, r} \bar{g}(x)=\bar{g}(y)$ for all $\bar{g} \in \bar{E}^{\leq r}$ with $x \in \operatorname{dom} \bar{g}$. Moreover $\bar{\phi}_{x, y, r}$ is non-expanding with respect to $d_{\bar{E}}$. Like $\phi$, we will simply use the notation $\bar{\phi}$ for $\bar{\phi}_{x, y, r}$. Note that $\bar{\phi}=\phi$ on $\bar{B}_{\bar{E}}(x, r / C) \cap U$, which is contained in $\bar{B}_{E}(x, r)$ by (8.1). Thus

$$
\bar{\phi}\left(\bar{B}_{\bar{E}}(x, r / C) \cap U\right) \subset \operatorname{im} \phi .
$$

On the other hand, for each $z \in \bar{B}_{E}(y, r / C)$, there is $g \in E^{\leq\lfloor r / C\rfloor}$ such that $y \in \operatorname{dom} g$ and $z=g(y)$. Hence $x \in \operatorname{dom} \bar{g}$ by (b), $\bar{g}(x) \in \bar{B}_{\bar{E}}(x, r / C)$ because $\bar{g} \in \bar{E}^{\leq\lfloor r / C\rfloor}$, and $\bar{\phi} \bar{g}(x)=\bar{g}(y)=z$ by (c). So

$$
\bar{B}_{E}(y, r / C) \subset \bar{\phi}\left(\bar{B}_{\bar{E}}(x, r / C)\right) .
$$

Assume that $r \geq C R$. Then $\bar{B}_{\bar{E}}(x, r / C) \cap U$ is a $2 R$-net in $\left(\bar{B}_{\bar{E}}(x, r / C), d_{\bar{E}}\right)$ by Lemma 3.4 . So $\bar{\phi}\left(\bar{B}_{\bar{E}}(x, r / C) \cap U\right)$ is a $2 R$-net in $\left(\bar{\phi}\left(\bar{B}_{\bar{E}}(x, r / C)\right), d_{\bar{E}}\right)$ because $\bar{\phi}$ is non-expanding. Hence $\bar{B}_{E}(y, r / C) \cap \operatorname{im} \phi$ is a $2 R$-net in $\left(\bar{B}_{E}(y, r / C), d_{\bar{E}}\right)$ by 8.2 and $(8.3)$, and therefore it is a $2 C R$-net in $\left(\bar{B}_{E}(y, r / C), d_{E}\right)$ by 8.1), showing vil. 
REMARK 8.12. Observe the following in Proposition 8.11.

(i) According to (ii), (ii) and (iii), $\left.\phi_{x, y, r^{\prime}}\right|_{\bar{B}_{E}(x, r)}=\phi_{x, y, r}$ if $r<r^{\prime}$ and $y \in V\left(x, r^{\prime}\right)$.

(ii) By (ii), (iii) and (iv), it follows that, if $s \geq r>0, x \in U_{0}, y \in V(x, r) \cap U_{0}$ and $z \in V(y, s) \cap V(x, r)$, then $\operatorname{im} \phi_{x, y, r} \subset \bar{B}_{E}(y, s)$ and $\phi_{x, z, s}=\phi_{y, z, s} \circ \phi_{x, y, r}$ on $\bar{B}_{E}(x, r)$.

REMARK 8.13. Let $U_{E}$ be the complement in $U$ of the $\mathcal{G}$-saturation of the union of boundaries in $U$ of the domains of the maps in $E$. Such a $U_{E}$ is a dense $G_{\delta}$ set, and thus so is $U_{0} \cap U_{E}$. For all $x \in U_{0} \cap U_{E}$, we can choose the open neighbourhoods $V(x, r)$ of Proposition 8.11 satisfying the conditions (ii) and (a) of Proposition 8.11 and its proof, and moreover so that:

- if $V(x, r)$ meets the domain of some $g \in E^{\leq r}$, then $x \in \operatorname{dom} g$; and,

- if $g(y)=y$ for some $g \in E^{\leq 4 r}$ and $y \in V(x, r) \cap \operatorname{dom} g$, then $x \in \operatorname{dom} g$ and $g(x)=x$.

Then, arguing like in the proof of Proposition 8.11, it is easy to prove that the maps $\phi_{x, y, r}$ are isometric bijections for all $r>0, x \in U_{0} \cap U_{E}$ and $y \in V(x, r)$.

The following weaker version of Proposition 8.11 is valid for all points of $U$.

Proposition 8.14. For every $r \in \mathbb{Z}^{+}$and $x \in U$, there exists an open neighborhood $W(x, r)$ of $x$ in $U$ such that:

(i) $W\left(x, r^{\prime}\right) \subset W(x, r)$ if $r^{\prime}>r$;

(ii) $x \in \operatorname{dom} \bar{g}$ for all $g \in E^{\leq r}$ and $y \in W(x, r) \cap \operatorname{dom} g$;

(iii) for every $y \in W(x, r)$, a map $\xi_{y, x, r}: \bar{B}_{E}(y, r) \rightarrow \bar{B}_{\bar{E}}(x, r)$ is determined by the condition $\xi_{y, x, r} g(y)=\bar{g}(x)$ for all $g \in E^{\leq r}$ with $y \in \operatorname{dom} g$;

(iv) $\xi_{y, x, r}$ is C-Lipschitz with respect to $d_{E}$; and

(v) $\bar{B}_{E}(x, r) \subset \operatorname{im} \xi_{y, x, r}$.

Proof. Like in the proof of Proposition 8.11. for every $r \in \mathbb{Z}^{+}$and $x \in U$, there is a largest open neighborhood $W(x, r)$ of $x$ in $U$ satisfying the following properties:

(a) $W(x, r) \subset \operatorname{dom} g$ for all $g \in E^{\leq r}$ with $x \in \operatorname{dom} g$;

(b) $x \in \operatorname{dom} \bar{g}$ for all $g \in E^{\leq 4 r}$ and $y \in W(x, r) \cap \operatorname{dom} g$; and

(c) if $g(y)=y$ for some $g \in E^{\leq 4 r}$ and $y \in W(x, r) \cap \operatorname{dom} g$, then $\bar{g}(x)=x$.

Property (i) is also satisfied because $W(x, r)$ is maximal.

Fix $x \in U, r \in \mathbb{Z}^{+}$and $y \in W(x, r)$. Property (b) is stronger than (ii). Each point in $\bar{B}_{E}(x, r)$ is of the form $g(x)$ for some $g \in E^{\leq r}$ with $y \in \operatorname{dom} g$, and therefore $x \epsilon$ $\operatorname{dom} \bar{g}$ by (a). Suppose that $g(y)=h(y)$ for another $h \in E^{\leq r}$ with $y \in \operatorname{dom} h$. Then $h^{-1} g \in E^{\leq 2 r}, y \in \operatorname{dom} h^{-1} g$ and $h^{-1} g(y)=y$. By (c), we get $x \in \operatorname{dom} \overline{h^{-1} g}=\bar{h}^{-1} \bar{g}$ and $\bar{h}^{-1} \bar{g}(x)=x$, obtaining $\bar{g}(x)=\bar{h}(x)$. Therefore a map $\xi_{y, x, r}: \bar{B}_{E}(y, r) \rightarrow \bar{B}_{E}(x, r)$ is defined by $g(y) \mapsto \bar{g}(x)$ for $g \in E^{\leq r}$, giving (iii).

For every $z, z^{\prime} \in \bar{B}_{E}(y, r)$ there are $g, g^{\prime} \in E^{\leq r}$ whose domains contain $y$ and such that $g(y)=z$ and $g^{\prime}(y)=z^{\prime}$. If $m=d_{E}\left(z, z^{\prime}\right) \leq 2 r$, there is $h \in E^{m}$ so that $z \in \operatorname{dom} h$ and $h\left(z^{\prime}\right)=z$. Thus $y \in \operatorname{dom} g^{-1} h g^{\prime}$ and $g^{-1} h g^{\prime}(y)=y$ with $g^{-1} h g^{\prime} \in E^{\leq 4 r}$. It follows from (b) and (c) that $x \in \operatorname{dom} \overline{g^{-1} h g^{\prime}}=\bar{g}^{-1} \bar{h} \bar{g}^{\prime}$ and $\bar{g}^{-1} \bar{h} \bar{g}^{\prime}(x)=x$. Hence $\bar{g}^{\prime}(x) \in \operatorname{dom} \bar{h}$ and $\bar{h} \bar{g}^{\prime}(x)=\bar{g}(x)$, giving $d_{\bar{E}}\left(\bar{g}(x), \bar{g}^{\prime}(x)\right) \leq m$. So

$$
d_{E}\left(\bar{g}(x), \bar{g}^{\prime}(x)\right) \leq C d_{\bar{E}}\left(\bar{g}(x), \bar{g}^{\prime}(x)\right) \leq C d_{E}\left(z, z^{\prime}\right)
$$

by (8.1), showing (iv). 
Let $z \in \bar{B}_{E}(x, r)$, and let $g \in E^{\leq r}$ be such that $z=g(x)$. Thus $y \in \operatorname{dom} g$ by (a), and $\xi_{y, x, r} g(y)=\bar{g}(x)=g(x)$, obtaining $\mathrm{v}$.

REMARK 8.15. In Proposition 8.14, note the following:

(i) By (ii), (ii) and (iii), $\left.\xi_{y, x, r^{\prime}}\right|_{\bar{B}_{E}(y, r)}=\xi_{y, x, r}$ if $r<r^{\prime}$ and $y \in W\left(x, r^{\prime}\right)$.

(ii) By (ii), (iii) and Proposition 8.14, (iii), (iii), $\xi_{y, x, r} \phi_{x, y, r}=$ id on $\bar{B}_{E}(x, r)$ if $x \in U_{0}$ and $y \in V(x, r) \cap W(x, r)$.

Proposition 8.16. For any convergent sequence in $U, x_{i} \rightarrow x$, and all $r \in \mathbb{Z}^{+}$,

$$
\bar{B}_{E}(x, r) \subset \bigcap_{i} \mathrm{Cl}_{U}\left(\bigcup_{j \geq i} \bar{B}_{E}\left(x_{i}, r\right)\right) \subset \bar{B}_{E}(x, C r) .
$$

Proof. Like in the proof of Proposition 8.11, for every $r \in \mathbb{Z}^{+}$and $x \in U$, there is a largest open neighbourhood $P$ of $x$ in $U$ such that the following properties hold for all $g \in E^{\leq r}$ :

(a) if $x \in \operatorname{dom} g$, then $P \subset \operatorname{dom} g$; and

(b) if $y \in P \cap \operatorname{dom} g$, then $x \in \operatorname{dom} \bar{g}$.

We can assume that $x_{i} \in P$ for all $i$.

The first inclusion of the statement can be proved as follows. Any element of $\bar{B}_{E}(x, r)$ is of the form $g(x)$ for some $g \in E^{\leq r}$. Then $g\left(x_{i}\right) \in \bar{B}_{E}\left(x_{i}, r\right)$ for all $i$ by (a), and $g\left(x_{i}\right) \rightarrow g(x)$ as $i \rightarrow \infty$.

Now let us prove the second inclusion. Consider a convergent sequence in $U$, $z_{k} \rightarrow z$, such that $z_{k} \in \bar{B}_{E}\left(x_{i_{k}}, r\right)$ for indices $i_{k} \rightarrow \infty$. Thus there are elements $g_{k} \in E^{\leq r}$ such that $x_{i_{k}} \in \operatorname{dom} g_{k}$ and $g_{k}\left(x_{i_{k}}\right)=z_{k}$. Since $E^{\leq r}$ is finite, by passing to a subsequence of $z_{k}$ if needed, we can assume that all maps $g_{k}$ are equal, and therefore they will be denoted by $g$. Then $x \in \operatorname{dom} \bar{g}$ by (b), and $z_{k}=\bar{g}\left(x_{i_{k}}\right) \rightarrow \bar{g}(x)$ as $k \rightarrow \infty$. Thus $z=\bar{g}(x) \in \bar{B}_{\bar{E}}(x, r) \subset \bar{B}_{E}(x, C r)$ by 8.1$)$.

\subsection{Topological dynamics}

8.4.1. Preliminaries on Baire category. We recall some terminology and results about subsets of a topological space that are relevant to topological dynamics. Good references for all this and related material are [71, [53, [14.

Definition 8.17. A subset $A$ of a topological space $X$ is called:

- residua $2^{2}$ if $A$ contains a countable intersection of open dense subsets;

- nowhere dense if its closure $\bar{A}$ has empty interior;

- meager if $A$ is a countable union of nowhere dense sets (i.e., $X \backslash A$ is residual);

- Borel if $A$ is a member of the $\sigma$-algebra generated by the open subsets of $X$; and

- Baire ${ }^{3}$ if the symmetric difference ${ }^{4} A \triangle U$ is meager for some open $U \subset X$.

The Baire sets of a topological space also form a $\sigma$-algebra: the smallest one containing all the open sets and all the meager sets; in particular, every Borel set is a Baire set. A topological space in which every residual subset is dense is called a Baire space. Any open subspace of a Baire space is a Baire space. The Baire

\footnotetext{
${ }^{2}$ The term comeager is also used.

${ }^{3}$ It is also said that $A$ satisfies the Baire property.

${ }^{4}$ Recall that the symmetric difference of the sets $A, B \subset X$ is the set $A \triangle B=(A \backslash B) \cup(B \backslash A)$.
} 
category theorem states that every completely metrizable space and every locally compact Hausdorff space is a Baire space [71, Theorem 8.4].

The Kuratowski-Ulam theorem is the topological analog to Fubini's theorem.

Theorem 8.18 (Kuratowski-Ulam; see e.g. [71, Theorem 8.41]). Let $X$ and $Y$ be second countable spaces, let $A \subset X \times Y$ be a Baire subset, and let $A_{x}=\{y \in X \mid$ $(x, y) \in A\}$ for each $x \in X$. Then the following properties hold:

(i) $A_{x}$ is Baire for residually many $x \in X$.

(ii) $A$ is meager (respectively, residual) if and only if $A_{x}$ is meager (respectively, residual) for residually many $x \in X$.

A topological space is called Polish if it is separable and completely metrizable; in particular, it is a Baire space. A subspace of a Polish space is Polish if and only if it is a $G_{\delta}$ [71, Theorem 3.11]. A locally compact space is Polish if and only if it is Hausdorff and second countable [71, Theorem 5.3].

8.4.2. Saturated sets. Let $\mathcal{H}$ be a pseudogroup on a space $Z$. A subset of $Z$ is said to be $\mathcal{H}$-saturated (or saturated) if it is a union of orbits of $\mathcal{H}$. The saturation of a subset $A \subset Z$, denoted by $\mathcal{H}(A)$, is the union of all orbits that meet $A$; i.e.,

$$
\mathcal{H}(A)=\bigcup_{h} h(A \cap \operatorname{dom} h),
$$

where $h$ runs in $\mathcal{H}$. If a property $P$ is satisfied by the $\mathcal{H}$-orbits in a residual (respectively, meager) saturated subset of $Z$, then it will be said that $P$ is satisfied by residually (respectively, meagerly) many $\mathcal{H}$-orbits.

Lemma 8.19. Let $A \subset B \subset Z$. If $A$ is open, dense or residual in $B$, then $\mathcal{H}(A)$ is open, dense or residual in $\mathcal{H}(B)$, respectively.

Proof. By 8.4), $\mathcal{H}(A)$ is open (respectively, dense) in $\mathcal{H}(B)$ if $A$ is open (respectively, dense) in $B$. It follows directly from this that $\mathcal{H}(A)$ is residual in $\mathcal{H}(B)$ if $A$ is residual in $B$.

Lemma 8.20. If $\mathcal{H}$ is countably generated, then the saturation of a Borel, Baire or meager subset of $Z$ is Borel, Baire or meager, respectively.

Proof. Let $E$ be a countable symmetric set of generators of $\mathcal{H}$, and let $S$ be the countable set of all composites of elements of $E$, wherever defined. Then the result follows because (8.4) still holds if $h$ runs only in $S$.

REMARK 8.21. Let $R_{\mathcal{H}} \subset Z \times Z$ be the relation set of the equivalence relation "being in the same $\mathcal{H}$-orbit." Assume that $\mathcal{H}$ is countably generated. With the notation of the proof of Lemma 8.20 $R_{\mathcal{H}}$ equals the union of the graphs of maps in $S$, which are easily seen to be $F_{\sigma}$-sets. Hence $R_{\mathcal{H}}$ is an $F_{\sigma}$ set because $S$ is countable.

A pseudogroup, $\mathcal{H}$, is called transitive (respectively, minimal) if it has a dense orbit (respectively, every orbit is dense). An $(\mathcal{H}$-) minimal set is a non-empty, saturated and closed subset of $Z$ which is minimal among the sets with these properties. The following result is well known.

Proposition 8.22. If $Z$ is second countable, then the union of all the $\mathcal{H}$-orbits that are dense is a $G_{\delta}$-set. In particular, this set is residual if and only if $\mathcal{H}$ is transitive. 
Proof. Let $\left\{U_{n}\right\}$ be a countable base for the topology of $Z$. Then the union of all the dense orbits is equal to the intersection of the saturations $\mathcal{H}\left(U_{n}\right)$, which are open in $Z$.

COROLlaRY 8.23. If $Z$ is second countable and $\mathcal{H}$ is transitive, then the union of all the proper minimal sets is meager.

Proposition 8.24. If $Z$ is locally compact space and $Z / \mathcal{H}$ is compact, then any nonempty $\mathcal{H}$-invariant closed subset of $Z$ contains some minimal set.

Proof. By Zorn's lemma, it is enough to prove that any family of $\mathcal{H}$-invariant nonempty closed subsets $Y_{i} \subset Z$ has nonempty intersection. By the hypothesis, there is a relatively compact open subset $U \subset Z$ that meets all $\mathcal{H}$-orbits. Then $\bar{U} \cap Y_{i}$ is a nonempty compact subset, obtaining $\varnothing \neq \bar{U} \cap \bigcap_{i} Y_{i} \subset \bigcap_{i} Y_{i}$.

The next result is the topological zero-one law, a topological version of ergodicity.

THEOREM 8.25. If $Z$ is a Baire space and $\mathcal{H}$ is a transitive pseudogroup on $Z$, then each saturated Baire subset of $Z$ is either meager or residual.

Proof. Let $A$ be a saturated Baire subset of $Z$. There is an open set $U$ such that $A \triangle U$ is a meager set. If $A$ is not meager, then $U$ is non-empty and $U \backslash A$ is meager. Thus, if $A$ is neither meager nor residual, then there are non-empty open subsets $U$ and $V$ so that $A \cap U$ is residual in $U$ and $V \backslash A$ is residual in $V$. Hence $\mathcal{H}(A \cap U)$ and $\mathcal{H}(V \backslash A)$ are residual in $\mathcal{H}(U)$ and $\mathcal{H}(V)$, respectively (Lemma 8.19). Since there is a dense orbit, the non-empty open sets $\mathcal{H}(U)$ and $\mathcal{H}(V)$ intersect in a non-empty set, and thus $\mathcal{H}(A \cap U)$ meets $\mathcal{H}(V \backslash A)$ because $\mathcal{H}(U)$ and $\mathcal{H}(V)$ are Baire spaces. But $\mathcal{H}(A \cap U) \subset A$ and $\mathcal{H}(V \backslash A) \subset Z \backslash A$, a contradiction.

The following theorem is of basic importance for the contents of this work.

TheOREm 8.26 (Hector [61, Epstein-Millet-Tischler 42]). If $\mathcal{H}$ is a countably generated pseudogroup on a space $Z$, then the union of orbits with trivial germ groups is a dense $G_{\delta}$ subset of $Z$, hence Borel and residual.

Corollary 8.27. If $Z$ is second countable, and $\mathcal{H}$ is transitive and countably generated, then the union of dense orbits with trivial germ groups is a residual subset of $Z$.

Proof. This is a direct consequence of Proposition 8.22 and Theorem 8.26 .

8.4.3. The property of being recurrent on Baire sets. The following shows that the condition of being recurrent on systems of compact generation also holds in a Baire sense. It is a topological-coarsely quasi-isometric version of Ghys' "Proposition fondamentale" [50, p. 402] for pseudogroups.

THEOREM 8.28. Let $\mathcal{H}$ be a compactly generated minimal pseudogroup of local transformations of a locally compact space $Z$, let $U$ be a relatively compact open subset of $Z$ that meets all $\mathcal{H}$-orbits, let $E$ be a recurrent symmetric system of compact generation of $\mathcal{H}$ on $U$, and let $\mathcal{G}=\left.\mathcal{H}\right|_{U}$. Then, for any Baire subset $B$ of $U$,

(i) either $\mathcal{G}(B)$ is meager;

(ii) or else the intersections of residually many $\mathcal{G}$-orbits with $B$ are equi-nets in those $\mathcal{G}$-orbits with $d_{E}$. 
Proof. Suppose $\mathcal{G}(B)$ is not meager in $U$. So $B$ is not meager in $U$ by Lemma 8.20. Then there is a non-empty open subset $V$ of $U$ such that $V \backslash B$ is meager in $V$. Hence $\mathcal{G}(V \backslash B)$ is meager in $U$ by Lemma 8.20 and thus $Y=$ $U \backslash \mathcal{G}(V \backslash B)$ is residual in $U$ and $\mathcal{G}$-saturated. Since $\mathcal{G}$ is minimal and $E$ recurrent, there is some $R>0$ such that $\mathcal{O} \cap V$ is an $R$-net in $\left(\mathcal{O}, d_{E}\right)$ for any $\mathcal{G}$-orbit $\mathcal{O}$ (Proposition 8.6); but $\mathcal{O} \cap V \subset B$ if $\mathcal{O} \subset Y$, and the result follows.

8.4.4. Pseudogroups versus group actions. As said in Section 8.1, a pseudogroup on a space is a generalization of a group acting on a space via homeomorphisms. With a slight change of the topology of the space acted on, the converse is also true, in the following sense.

THEOREM 8.29. Let $\mathcal{H}$ be a countable generated pseudogroup of local homeomorphisms of a Polish space $Z$. Then there is a Polish space $Z^{\prime}$, with the same underlying set as $Z$, and a pseudogroup $\mathcal{H}^{\prime}$ on $Z^{\prime}$ such that $\mathcal{H}^{\prime}$ has the same orbits as $\mathcal{H}, \mathcal{H} \subset \mathcal{H}^{\prime}$, and $\mathcal{H}^{\prime}$ is equivalent to the pseudogroup generated by a countable group $G$ of homeomorphisms on another Polish space. Moreover, for each symmetric set $E$ of generators of $\mathcal{H}$, there is a symmetric set $F$ of generators of $G$, with the same cardinality as $E$, such that the $\mathcal{H}$-orbits with $d_{E}$ are equi-coarsely quasi-isometric to the corresponding $G$-orbits with $d_{F}$.

Proof. Let $W=Z \sqcup Z=Z \times\{0,1\}$. For $i \in\{0,1\}$, let $\iota_{i}: Z \rightarrow W$ be given by $\iota_{i}(z)=(z, i)$, and let $Z_{i}=\iota_{i}(Z)$. Let $E$ be a countable symmetric set of generators for $\mathcal{H}$ containing $\operatorname{id}_{Z}$. For each $h \in E$, let $g_{h}$ be the Borel measurable bijection of $W$ given by $g_{h} \iota_{0}(z)=\iota_{1} h(z)$ if $z \in \operatorname{dom} h, g_{h} \iota_{1}(z)=\iota_{0} h^{-1}(z)$ if $z \in \operatorname{im} h$, and $g_{h} \iota_{i}(z)=\iota_{i}(z)$ otherwise; in particular, $g_{\mathrm{id}_{z}} \iota_{0}(z)=\iota_{1}(z)$ and $g_{\mathrm{id}_{z}} \iota_{1}(z)=\iota_{0}(z)$ for all $z \in Z$. Then $F=\left\{g_{h} \mid h \in E\right\}$ generates a countable group $G$ of Borel measurable bijections of $W$. Note that $F$ has the same cardinality as $E$, and is symmetric because every $g_{h}$ is of order 2 .

By [15, Theorem 5.2.1], there is a Polish topology $\tau^{*}$ on $W$ so that $G$ consists of homeomorphisms of the corresponding space $W^{*}$, and inducing the same Borel $\sigma$-algebra as the original topology of $W$. Writing $E=\left\{h_{n} \mid n \in \mathbb{N}\right\}$, by [15. Theorem 5.1.11], there are Polish topologies $\tau_{n}$ on $W$ such that $\tau^{*} \subset \tau_{0} \subset \tau_{1} \subset \cdots$, $\iota_{0}\left(\operatorname{dom} h_{n}\right) \in \tau_{n}$, and $G$ consists of homeomorphisms of the corresponding spaces. By [15. Theorem 5.1.3-(b)], the topology $\tau^{\prime}$ generated by $\bigcup_{n} \tau_{n}$ is also Polish. Since $\cup_{n} \tau_{n}$ is a base of $\tau^{\prime}$, the maps in $G$ are homeomorphisms of the space $W^{\prime}$ defined with $\tau^{\prime}$. Let $\mathcal{G}$ be the pseudogroup generated by $G$ on $W^{\prime}$.

Since $Z_{0}, Z_{1} \in \tau^{\prime}$ and $g_{\mathrm{id} Z}$ restricts to a homeomorphism $Z_{0} \rightarrow Z_{1}$, there is a Polish topology on $Z$ so that the corresponding space $Z^{\prime}$ satisfies $Z^{\prime} \sqcup Z^{\prime}=W^{\prime}$. Thus the maps $\iota_{i}: Z^{\prime} \rightarrow W^{\prime}$ are open embeddings, the sets $Z_{i}$ meet all $G$-orbits, and there is a unique pseudogroup $\mathcal{H}^{\prime}$ on $Z^{\prime}$ so that any $\iota_{i}$ generates an equivalence $\mathcal{H}^{\prime} \rightarrow \mathcal{G}$.

For each $h \in E$, the restriction $g_{\mathrm{id}_{z}} g_{h}: \iota_{0}(\operatorname{dom} h) \rightarrow \iota_{0}(\operatorname{im} h)$ is in $\mathcal{H}^{\prime}$ and corresponds to $h$ via $\iota_{0}$. Thus $E \subset \mathcal{H}^{\prime}$, and therefore $\mathcal{H} \subset \mathcal{H}^{\prime}$; in particular, the $\mathcal{H}$-orbits are contained in $\mathcal{H}^{\prime}$-orbits.

Suppose that $\mathcal{O} \subset \mathcal{O}^{\prime}$ for orbits $\mathcal{O}$ of $\mathcal{H}$ and $\mathcal{O}^{\prime}$ of $\mathcal{H}^{\prime}$. For different points, $z \in \mathcal{O}$ and $z^{\prime} \in \mathcal{O}^{\prime}$, let $k=d_{F}\left(\iota_{0}(z), \iota_{0}\left(z^{\prime}\right)\right) \geq 1$, and take $h_{1}, \ldots, h_{k} \in E$ so that

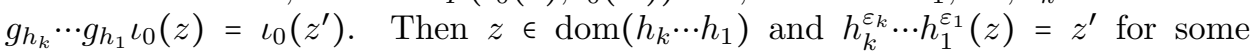
choice of $\varepsilon_{1}, \ldots, \varepsilon_{k} \in\{ \pm 1\}$, obtaining that $z^{\prime} \in \mathcal{O}$ and $d_{E}\left(z, z^{\prime}\right) \leq k$. In particular, this shows that $\mathcal{O}=\mathcal{O}^{\prime}$. Now let $l=d_{E}\left(z, z^{\prime}\right) \geq 1$, and take $\bar{h}_{1}, \ldots, \bar{h}_{l} \in E$ such 
that $z \in \operatorname{dom}\left(\bar{h}_{l} \cdots \bar{h}_{1}\right)$ and $\bar{h}_{l} \cdots \bar{h}_{1}(z)=z^{\prime}$. Then, either $g_{\bar{h}_{l}^{\delta_{l}}} \cdots g_{\bar{h}_{1}^{\delta_{1}}} \iota_{0}(z)=\iota_{0}\left(z^{\prime}\right)$, or $g_{\mathrm{id}_{z}} g_{\bar{h}_{l}^{\delta_{l}}} \cdots g_{\bar{h}_{1}^{\delta_{1}}} \iota_{0}(z)=\iota_{0}\left(z^{\prime}\right)$, for some choices of $\delta_{1}, \ldots, \delta_{l} \in\{ \pm 1\}$, obtaining $k \leq l+1$. On the other hand, the intersections of the $G$-orbits with $Z_{0}$ are 1-nets in those $G$-orbits with $d_{F}$ because $g_{\mathrm{id}_{Z}}\left(Z_{1}\right)=Z_{0}$. So $\iota_{0}$ defines equi-corse quasi-isometries of the $\mathcal{H}$-orbits with $d_{E}$ to the corresponding $G$-orbits with $d_{F}$.

REMARK 8.30. (i) The doubling of the space is required because the domain and image of a local homeomorphisms $h \in E$ may have non-empty intersection. However, if $\mathcal{H}$ is the representative of the holonomy pseudogroup of a foliated space generated by the transition mappings of a regular foliated atlas (Section 10.1), then that doubling is not necessary because each transition mapping has disjoint image and domain.

(ii) Theorem 8.29 is related to the result of Feldman and Moore [45, Theorem 1] stating the following: If $R$ countable equivalence relation on a standard Borel space $X$, then there is a countable group $G$ of Borel automorphisms of $X$ so that $R$ is the orbit equivalence relation induced by $G$. 



\section{CHAPTER 9}

\section{Generic coarse geometry of orbits}

The following notation will be used in the whole of this chapter.

Hypothesis 1 . A quintuple $(Z, \mathcal{H}, U, \mathcal{G}, E)$ is required to satisfy the following conditions:

- $Z$ is a locally compact Polish space,

- $\mathcal{H}$ is a compactly generated pseudogroup of local transformations of $Z$,

- $U$ is a relatively compact open subset of $Z$ that meets all $\mathcal{H}$-orbits,

- $\mathcal{G}$ denotes the restriction of $\mathcal{H}$ to $U$, and

- $E$ is a recurrent symmetric system of compact generation of $\mathcal{H}$ on $U$.

All metric concepts in the $\mathcal{G}$-orbits will be considered with respect to the metric $d_{E}$ induced by $E$. Thus the subindex " $E$ " will be deleted from the notation of the metric, open balls, closed balls, and penumbras. Let $U_{0}$ denote the union of $\mathcal{G}$-orbits with trivial germ groups, which is a dense $G_{\delta}$-subset of $U$ by Theorem 8.26, and therefore $U_{0}$ is a Polish subspace (Section 8.4.1). Moreover let $U_{0, \mathrm{~d}}$ be the union of dense orbits in $U_{0}$, which is a residual subset of $Z$ if $\mathcal{H}$ is transitive (Corollary 8.27). For each $A \subset U_{0}$, let $\mathrm{Cl}_{0}(A)$ and $\operatorname{Int}_{0}(A)$ denote its closure and interior in $U_{0}$, respectively; the same notation will be used for the closure and interior in $U_{0} \times U_{0}$. Assume that $|E| \geq 2$, otherwise the $\mathcal{G}$-orbits have at most two elements.

\subsection{Coarsely quasi-isometric orbits}

For $K \in \mathbb{N}$ and $C \in \mathbb{Z}^{+}$, let $Y(K, C)$ be the set of pairs $(x, y) \in U_{0} \times U_{0}$ such that there is a $(K, C)$-coarse quasi-isometry $f: A \rightarrow B$ of $\mathcal{G}(x)$ to $\mathcal{G}(y)$ with $x \in A$, $y \in B$ and $f(x)=y$. Notice that $Y(K, C) \subset Y\left(K^{\prime}, C^{\prime}\right)$ if $K \leq K^{\prime}$ and $C \leq C^{\prime}$.

Lemma 9.1. For $K \in \mathbb{N}$ and $C \in \mathbb{Z}^{+}$, there is some $K^{\prime} \in \mathbb{N}$ and $C^{\prime} \in \mathbb{Z}^{+}$, depending only on $K$ and $C$, such that $\mathrm{Cl}_{0}(Y(K, C)) \subset Y\left(K^{\prime}, C^{\prime}\right)$.

Proof. Let $(x, y) \in \mathrm{Cl}_{0}(Y(K, C))$. For each $r \in \mathbb{Z}^{+}$, consider the open neighborhoods $V(x, r)$ and $V(y, r)$ of $x$ and $y$ in $U$ given by Proposition 8.11. Then there is some

$$
\left(x_{n}, y_{n}\right) \in Y(K, C) \cap(V(x, n) \times V(y, C n))
$$

for each $n \in \mathbb{Z}^{+}$. According to Proposition 8.11, by taking $K$ and $C$ large enough, it can be assumed that all $\phi_{n}:=\phi_{x, x_{n}, n}$ and $\psi_{n}:=\phi_{y, y_{n}, C n}$ are non-expanding equi-biLipschitz maps with bi-Lipschitz distortion $C$, and the sets $\bar{B}\left(x_{n}, n / C\right) \cap \operatorname{im} \phi_{n}$ and $\bar{B}\left(y_{n}, n\right) \cap \operatorname{im} \psi_{n}$ are $K$-nets in $\bar{B}\left(x_{n}, n / C\right)$ and $\bar{B}\left(y_{n}, n\right)$, respectively. In particular, the restriction

$$
\phi_{n}: \phi_{n}^{-1}\left(\bar{B}\left(x_{n}, n / C\right)\right) \rightarrow \bar{B}\left(x_{n}, n / C\right) \cap \operatorname{im} \phi_{n}
$$

is a $(K, C)$-coarse quasi-isometry of $\phi_{n}^{-1}\left(\bar{B}\left(x_{n}, n / C\right)\right)$ to $\bar{B}\left(x_{n}, n / C\right)$. 
On the other hand, for each $n$, there is a $(K, C)$-coarse quasi-isometry $f_{n}$ : $A_{n} \rightarrow B_{n}$ of $\mathcal{G}\left(x_{n}\right)$ to $\mathcal{G}\left(y_{n}\right)$, and so that $x_{n} \in A_{n}, y_{n} \in B_{n}$ and $f_{n}\left(x_{n}\right)=y_{n}$. Each set $\bar{B}\left(x_{n}, n / C\right) \cap A_{n}$ is a $2 K$-net in $\bar{B}\left(x_{n}, n / C\right)$; this holds for $\lfloor n / C\rfloor>K$ by Lemma 3.4 and for $\lfloor n / C\rfloor \leq K$ because $x_{n} \in \bar{B}\left(x_{n}, n / C\right) \cap A_{n}$. Also, note that

$$
f_{n}\left(\bar{B}\left(x_{n}, n / C\right) \cap A_{n}\right) \subset \bar{B}\left(y_{n}, n\right),
$$

and thus each $f_{n}\left(\bar{B}\left(x_{n}, n / C\right) \cap A_{n}\right)$ is a $2 K$-net in its $2 K$-penumbra $P_{n}$ in $\bar{B}\left(y_{n}, n\right)$, obtaining that each restriction

$$
f_{n}: \bar{B}\left(x_{n}, n / C\right) \cap A_{n} \rightarrow f_{n}\left(\bar{B}\left(x_{n}, n / C\right) \cap A_{n}\right)
$$

is a $(2 K, C)$-coarse quasi-isometry of $\bar{B}\left(x_{n}, n / C\right)$ to $P_{n}$. Moreover, because each $\bar{B}\left(y_{n}, n\right) \cap \operatorname{im} \psi_{n}$ is a $K$-net in $\bar{B}\left(y_{n}, n\right)$, each $P_{n} \cap \operatorname{im} \psi_{n}$ is a $2 K$-net in $P_{n}$ by Lemma 3.4 So the restriction $\psi_{n}^{-1}: P_{n} \cap \operatorname{im} \psi_{n} \rightarrow \psi_{n}^{-1}\left(P_{n}\right)$ is a $(2 K, C)$-coarse quasi-isometry of $P_{n}$ to $\psi_{n}^{-1}\left(P_{n}\right)$. It follows from Proposition 2.12 that, for some $K^{\prime} \geq 0$ and $C^{\prime} \geq 1$, depending only on $K$ and $C$, there is a $\left(K^{\prime}, C^{\prime}\right)$-coarse quasiisometry $g_{n}$ of $\phi_{n}^{-1}\left(\bar{B}\left(x_{n}, n / C\right)\right)$ to $\psi_{n}^{-1}\left(P_{n}\right)$; this $g_{n}$ is a coarse composite of the above three coarse quasi-isometries. Since

$$
\begin{gathered}
x \in \phi_{n}^{-1}\left(\bar{B}\left(x_{n}, n / C\right)\right), \quad \phi_{n}(x)=x_{n} \in \bar{B}\left(x_{n}, n / C\right) \cap A_{n}, \\
f_{n}\left(x_{n}\right)=y_{n} \in P_{n} \cap \operatorname{im} \psi_{n}, \quad \psi_{n}^{-1}\left(y_{n}\right)=y,
\end{gathered}
$$

Proposition 2.12 also guarantees that $g_{n}$ can be chosen so that $x \in \operatorname{dom} g_{n}, y \in \operatorname{im} g_{n}$ and $g_{n}(x)=y$.

Observe that

$$
\bar{B}(x, n / C) \subset \phi_{n}^{-1}\left(\bar{B}\left(x_{n}, n / C\right)\right)
$$

because $\phi_{n}$ is non-expanding and $\phi_{n}(x)=x_{n}$. Therefore the sequence of finite sets $\phi_{n}^{-1}\left(\bar{B}\left(x_{n}, n / C\right)\right)$ is exhausting in $\mathcal{G}(x)$. On the other hand,

$$
\bar{B}\left(y_{n}, n / C^{2}\right) \cap B_{n} \subset f_{n}\left(\bar{B}\left(x_{n}, n / C\right) \cap A_{n}\right)
$$

since $f_{n}: A_{n} \rightarrow B_{n}$ is a $C$-bi-Lipschitz bijection such that $x_{n} \in A_{n}, y_{n} \in B_{n}$ and $f_{n}\left(x_{n}\right)=y_{n}$. Furthermore $\bar{B}\left(y_{n}, n / C^{2}\right) \cap B_{n}$ is a $2 K$-net in $\bar{B}\left(y_{n}, n / C^{2}\right)$, which holds for $\left\lfloor n / C^{2}\right\rfloor>K$ by Lemma 3.4 and for $\left\lfloor n / C^{2}\right\rfloor \leq K$ because $y_{n} \epsilon$ $\bar{B}\left(y_{n}, n / C^{2}\right) \cap B_{n}$. Therefore

$$
P_{n} \supset \bar{B}\left(y_{n}, n\right) \cap \operatorname{Pen}\left(\bar{B}\left(y_{n}, n / C^{2}\right) \cap B_{n}, 2 K\right) \supset \bar{B}\left(y_{n}, n / C^{2}\right),
$$

giving

$$
\bar{B}\left(y, n / C^{2}\right) \subset \psi_{n}^{-1}\left(\bar{B}\left(y_{n}, n / C^{2}\right)\right) \subset \psi_{n}^{-1}\left(P_{n}\right)
$$

since $\psi_{n}$ is non-expanding and $\psi_{n}(y)=y_{n}$. So the sequence of finite sets $\psi_{n}^{-1}\left(P_{n}\right)$ is exhausting in $\mathcal{G}(y)$.

By applying Proposition 2.15 to the sequence of coarse quasi-isometries $g_{n}$, and using Remark 2.16, it follows that there is a $\left(K^{\prime}, C^{\prime}\right)$-coarse quasi-isometry $g$ of $\mathcal{G}(x)$ to $\mathcal{G}(y)$ such that $x \in \operatorname{dom} g$ and $g(x)=y$; i.e., $(x, y) \in Y\left(K^{\prime}, C^{\prime}\right)$.

REMARK 9.2. If the statement of Lemma 9.1 were restricted to the residual union of orbits which do not meet the boundaries in $U$ of the domains of maps in $E$, then it would be an easy consequence of Proposition 2.15 and Remark 8.13 .

Let $Y$ be the set of points $(x, y) \in U_{0} \times U_{0}$ such that $\mathcal{G}(x)$ is coarsely quasiisometric to $\mathcal{G}(y)$.

Lemma 9.3. $Y=\cup_{K, C=1}^{\infty} Y(K, C)$. 
Proof. This equality follows from Corollary 2.33.

Corollary 9.4. $Y=\bigcup_{K, C=1}^{\infty} \mathrm{Cl}_{0}(Y(K, C))$; in particular, $Y$ is an $F_{\sigma}$-subset of $U_{0} \times U_{0}$.

Proof. This is elementary by Lemmas 9.1 and 9.3 .

Corollary 9.5. Either $\operatorname{Int}_{0}(Y(K, C)) \neq \varnothing$ for some $K, C \in \mathbb{Z}^{+}$, or else $Y$ is a meager subset of $U_{0} \times U_{0}$.

Proof. If $\operatorname{Int}_{0}(Y(K, C))=\varnothing$ for all $K, C \in \mathbb{Z}^{+}$, then $\operatorname{Int}_{0}\left(\mathrm{Cl}_{0}(Y(K, C))\right)=\varnothing$ for all $K, C \in \mathbb{Z}^{+}$by Lemma 9.1 and thus $Y$ is meager in $U_{0} \times U_{0}$ by Corollary 9.4 .

Theorem 9.6. Let $(Z, \mathcal{H}, U, \mathcal{G}, E)$ satisfy Hypothesis 1 . The equivalence relation " $x \sim y$ if and only if the orbits $\mathcal{G}(x)$ and $\mathcal{G}(y)$ are coarsely quasi-isometric" has a Borel relation set in $U_{0} \times U_{0}$, and has a Baire relation set in $U \times U$; in particular, it has Borel equivalence classes in $U_{0}$, and Baire equivalence classes in $U$.

Proof. This follows from Corollary 9.4 and Theorem 8.26 .

Theorem 9.7. Let $(Z, \mathcal{H}, U, \mathcal{G}, E)$ satisfy Hypothesis 1 . Suppose that $\mathcal{H}$ is transitive. Then:

(i) either all $\mathcal{G}$-orbits in $U_{0, \mathrm{~d}}$ are equi-coarsely quasi-isometric to each other;

(ii) or else every $\mathcal{G}$-orbit is coarsely quasi-isometric to meagerly many $\mathcal{G}$-orbits; in particular, there are uncountably many coarse quasi-isometry types of $\mathcal{G}$-orbits in $U_{0}$ in this case.

Proof. Suppose that $\operatorname{Int}_{0}(Y(K, C)) \neq \varnothing$ for some $K, C \in \mathbb{Z}^{+}$. Then all dense $\mathcal{G} \times \mathcal{G}$-orbits in $U_{0} \times U_{0}$ meet $Y(K, C)$, and thus all $\mathcal{G}$-orbits in $U_{0, \mathrm{~d}}$ are equi-coarsely quasi-isometric to each other.

If $\operatorname{Int}_{0}(Y(K, C))=\varnothing$ for all $K, C \in \mathbb{Z}^{+}$, then $Y$ is meager in $U_{0} \times U_{0}$ by Corollary 9.5. It follows from Theorem 8.18 that there is a residual subset $A \subset U_{0}$ such that $Y_{x}=\left\{y \in U_{0} \mid(x, y) \in Y\right\}$ is meager in $U$ for all $x \in A$. But each $Y_{x}$ is the union of orbits in $U_{0}$ which are coarsely quasi-isometric to $\mathcal{G}(x)$. Finally, $A$ can be assumed to be $\mathcal{G}$-saturated since $Y$ is $\mathcal{G} \times \mathcal{G}$-saturated.

TheOREM 9.8. Let $(Z, \mathcal{H}, U, \mathcal{G}, E)$ satisfy Hypothesis 1 . Then the following properties hold:

(i) Suppose that $\mathcal{H}$ is transitive. If there is a coarsely quasi-symmetric $\mathcal{G}$-orbit in $U_{0, \mathrm{~d}}$, then the alternative (i) of Theorem 9.7 holds.

(ii) Suppose that $\mathcal{H}$ is minimal. If the alternative (ii) of Theorem 9.7 holds, then all $\mathcal{G}$-orbits in $U_{0}$ are equi-coarsely quasi-symmetric.

Proof. Suppose that $\mathcal{H}$ is transitive and that there is a coarsely quasi-symmetric $\mathcal{G}$-orbit $\mathcal{O}$ in $U_{0, \mathrm{~d}}$. Then $\mathcal{O} \times \mathcal{O} \subset Y(K, C)$ for some $K, C \in \mathbb{Z}^{+}$, giving $U_{0} \times U_{0}=$ $Y\left(K^{\prime}, C^{\prime}\right)$ for $K^{\prime}, C^{\prime} \in \mathbb{Z}^{+}$by Lemma 9.1, which means that all $\mathcal{G}$-orbits in $U_{0}$ are equi-coarsely quasi-isometric to each other.

Assume that $\mathcal{H}$ is minimal and that all $\mathcal{G}$-orbits in $U_{0}$ are coarsely quasiisometric to each other. This means that $Y=U_{0} \times U_{0}$. Hence $\operatorname{Int}_{0}(Y(K, C)) \neq \varnothing$ for some $K, C \in \mathbb{Z}^{+}$by Corollary 9.5 i.e., there are some non-empty open subsets $V$ and $W$ of $U_{0}$ so that $V \times W \subset Y(K, C)$. But, by Proposition 8.6 and since $\mathcal{G}$ is minimal, the intersections $\mathcal{O} \cap V$ and $\mathcal{O} \cap W$ are equi-nets in the $\mathcal{G}$-orbits $\mathcal{O}$ in $U_{0}$. So the $\mathcal{G}$-orbits in $U_{0}$ are equi-coarsely quasi-symmetric by Lemma 3.18 , 
The following result follows from Theorems 9.8 and 6.39 .

Corollary 9.9. Let $(Z, \mathcal{H}, U, \mathcal{G}, E)$ satisfies Hypothesis 1 . Suppose that $\mathcal{G}$ is minimal and satisfies the alternative (i) of Theorem 9.7. Then all $\mathcal{G}$-orbits in $U_{0}$ have zero, one, two or a Cantor space of coarse ends, simultaneously.

\subsection{Growth of the orbits}

9.2.1. Orbits with the same growth type. Since the $\mathcal{G}$-orbits are equiquasi-lattices in themselves (Example 3.7-(i)), the growth type of $\mathcal{G}(x)$ is represented by the mapping $r \mapsto v(x, r)=|\overline{B(x, r)}|$ for all $x \in U$. For $a, b \in \mathbb{Z}^{+}$and $c \in \mathbb{N}$, let

$$
Y(a, b, c)=\left\{(x, y) \in U_{0} \times U_{0} \mid v(x, r) \leq a v(y, b r) \forall r \geq c\right\}
$$

Note that

$$
a \leq a^{\prime}, b \leq b^{\prime}, c \leq c^{\prime} \Longrightarrow Y(a, b, c) \subset Y\left(a^{\prime}, b^{\prime}, c^{\prime}\right) .
$$

LemmA 9.10. For all $a, b \in \mathbb{Z}^{+}$and $c \in \mathbb{N}$, there are some integers $a^{\prime} \geq a$ and $b^{\prime} \geq b$ such that $\mathrm{Cl}_{0}(Y(a, b, c)) \subset Y\left(a^{\prime}, b^{\prime}, c\right)$.

Proof. Consider the notation of Proposition 8.11. Let $(x, y) \in \mathrm{Cl}_{0}(Y(a, b, c))$. For any integer $r \geq c$, take a pair

$$
\left(x^{\prime}, y^{\prime}\right) \in Y(a, b, c) \cap(V(x, r) \times V(y, C b r)) .
$$

Then, with the notation given by 3.3 ,

$$
v(x, r) \leq v\left(x^{\prime}, r\right) \leq a v\left(y^{\prime}, b r\right) \leq a \Lambda_{|E|, 2 R C} v(y, C b r)
$$

since $\phi_{x, x^{\prime}, r}$ is injective (Proposition $8.11(\mathrm{v})$ ) and $\bar{B}\left(y^{\prime}, b r\right) \cap \operatorname{im} \phi_{y, y^{\prime}, C b r}$ is a $2 R C$ net in $\bar{B}\left(y^{\prime}, b r\right)$ (Proposition 8.11-vi)), and because $|\bar{B}(z, 2 R C)| \leq \Lambda_{|E|, 2 R C}$ for all $z \in \bar{B}\left(y^{\prime}, b r\right)$ by (3.4). Hence $(x, y) \in Y\left(a \Lambda_{|E|, 2 R C}, C b, c\right)$.

Note that $Y=\bigcup_{a, b, c=1}^{\infty} Y(a, b, c)$ is the set of points $(x, y) \in U_{0} \times U_{0}$ such that the growth type of $\mathcal{G}(x)$ is dominated by the growth type of $\mathcal{G}(y)$. Let $\tau: U \times U \rightarrow U \times U$ be the homeomorphism given by $\tau(x, y)=(y, x)$

THEOREM 9.11. Let $(Z, \mathcal{H}, U, \mathcal{G}, E)$ satisfy Hypothesis 1 . The equivalence relation " $x \sim y$ if and only if the orbits $\mathcal{G}(x)$ and $\mathcal{G}(y)$ have the same growth type" has a Borel relation set in $U_{0} \times U_{0}$, and has a Baire relation set in $U \times U$; in particular, it has Borel equivalence classes in $U_{0}$, and Baire equivalence classes in $U$.

Proof. By Lemma 9.10, $Y$ is an $F_{\sigma}$-subset of $U_{0} \times U_{0}$, and thus so is $Y_{\tau}:=$ $Y \cap \tau(Y)$. But $Y_{\tau}$ is the relation set in $U_{0} \times U_{0}$ of the statement, and the result follows because $U_{0}$ is residual in $U$ (Theorem 8.26). then:

TheOREM 9.12. Let $(Z, \mathcal{H}, U, \mathcal{G}, E)$ satisfy Hypothesis 1 . If $\mathcal{G}$ is transitive,

(i) either all $\mathcal{G}$-orbits in $U_{0, \mathrm{~d}}$ have equi-equivalent growth;

(ii) or else the growth type of every $\mathcal{G}$-orbit is comparable with the growth type of meagerly many $\mathcal{G}$-orbits; in particular, there are uncountably many growth types of $\mathcal{G}$-orbits in $U_{0}$ in this second case. 
Proof. If $\operatorname{Int}_{0}(Y(a, b, c)) \neq \varnothing$ for some $a, b, c \in \mathbb{Z}^{+}$, then all dense $\mathcal{G} \times \mathcal{G}$-orbits in $U_{0} \times U_{0}$ meet $Y(a, b, c)$. It follows that all $\mathcal{G}$-orbits in $U_{0, \mathrm{~d}}$ have equi-equivalent growth.

On the other hand, if $\operatorname{Int}_{0}(Y(a, b, c))=\varnothing$ for all $a, b, c \in \mathbb{Z}^{+}$, then $Y$ is a meager subset of $U_{0} \times U_{0}$ by Lemma 9.10, and thus it is meager in $U \times U$ too. So $Y^{\tau}:=$ $Y \cup \tau(Y)$ is meager in $U \times U$ as well. It follows from Theorem 8.18 that there is a residual subset $A \subset U_{0}$ such that $Y_{x}^{\tau}=\left\{y \in U_{0} \mid(x, y) \in Y^{\tau}\right\}$ is meager for all $x \in A$. But each $Y_{x}^{\tau}$ is the union of $\mathcal{G}$-orbits in $U_{0}$ whose growth type is comparable with the growth type of $\mathcal{G}(x)$. Obviously, it can be assumed that $A$ is saturated.

Theorem 9.13. Let $(Z, \mathcal{H}, U, \mathcal{G}, E)$ satisfy Hypothesis 1 . Then the following properties hold:

(i) Suppose that $\mathcal{H}$ is transitive. If there is a growth symmetric $\mathcal{G}$-orbit in $U_{0, \mathrm{~d}}$, then the alternative (i) of Theorem 9.12 holds.

(ii) Suppose that $\mathcal{H}$ is minimal. If the alternative (i) of Theorem 9.12 holds, then all $\mathcal{G}$-orbits in $U_{0}$ are equi-growth symmetric.

Proof. Suppose that $\mathcal{G}$ is transitive and that $\mathcal{G}(x)$ is growth symmetric for some $x \in U_{0, \mathrm{~d}}$. Then $\mathcal{G}(x) \times \mathcal{G}(x) \subset Y(a, b, c)$ for some $a, b \geq 1$ and $c \geq 0$, giving $U_{0} \times U_{0}=Y\left(a^{\prime}, b^{\prime}, c\right)$ for some $a^{\prime}, b^{\prime} \geq 1$ by Lemma 9.10 .

Now, assume that $\mathcal{G}$ is minimal and that all $\mathcal{G}$-orbits in $U_{0}$ have equi-equivalent growth. This means that $\operatorname{Int}_{0}(Y(a, b, c)) \neq \varnothing$ for some $a, b, c \in \mathbb{Z}^{+}$according to the proof of Theorem 9.12 i.e., there are non-empty open subsets $V$ and $W$ of $U_{0}$ such that $V \times W \subset Y(a, b, c)$. Since $\mathcal{G}$ is minimal, the intersections $\mathcal{O} \cap V$ and $\mathcal{O} \cap W$ are equi-nets in the $\mathcal{G}$-orbits $\mathcal{O}$ in $U_{0}$ by Proposition 8.6. So the $\mathcal{G}$-orbits in $U_{0}$ is equi-growth symmetric by Remark 4.14 (iii).

\subsubsection{Some growth classes of the orbits.}

Theorem 9.14. Let $(Z, \mathcal{H}, U, \mathcal{G}, E)$ satisfy Hypothesis 1 and suppose that $\mathcal{G}$ is transitive. Then there are $a_{1}, a_{3} \in[1, \infty], a_{2}, a_{4} \in[0, \infty)$ and $p \geq 1$ such that

$$
\begin{array}{rrrl}
\limsup _{r \rightarrow \infty} \frac{\log v(x, r)}{\log r}=a_{1}, & a_{2} \leq \liminf _{r \rightarrow \infty} \frac{\log v(x, r)}{r} \leq p a_{2}, \\
\liminf _{r \rightarrow \infty} \frac{\log v(x, r)}{\log r}=a_{3}, & \limsup _{r \rightarrow \infty} \frac{\log v(x, r)}{r}=a_{4}
\end{array}
$$

for residually many points $x$ in $U$. Moreover

$$
\liminf _{r \rightarrow \infty} \frac{\log v(x, r)}{\log r} \geq a_{3}, \quad \limsup _{r \rightarrow \infty} \frac{\log v(x, r)}{r} \leq a_{4}
$$

for all $x \in U_{0, \mathrm{~d}}$.

Proof. For $a, b>0$, let $Y_{1}(a, b), Y_{2}(a, b), Y_{3}(a, b)$ and $Y_{4}(a, b)$ be the sets of points $x \in U_{0}$ that satisfy the following respective conditions:

$$
\begin{array}{ll}
\sup _{r \geq b} \frac{\log v(x, r)}{\log r} \leq a, & \inf _{r \geq b} \frac{\log v(x, r)}{r} \geq a, \\
\inf _{r \geq b} \frac{\log v(x, r)}{\log r}<a, & \sup _{r \geq b} \frac{\log v(x, r)}{r}>a .
\end{array}
$$


Then

$$
a \leq a^{\prime} \& b \leq b^{\prime} \Longrightarrow\left\{\begin{aligned}
Y_{1}(a, b) \subset Y_{1}\left(a^{\prime}, b^{\prime}\right), & Y_{2}\left(a^{\prime}, b\right) \subset Y_{2}\left(a, b^{\prime}\right), \\
Y_{3}\left(a, b^{\prime}\right) \subset Y_{3}\left(a^{\prime}, b\right), & Y_{4}\left(a^{\prime}, b^{\prime}\right) \subset Y_{4}(a, b),
\end{aligned}\right.
$$

Claim 10. The following properties hold:

(i) $Y_{1}(a, b)$ is closed in $U_{0}$ for all $a, b>0$.

(ii) There is some $p \geq 1$ such that, for all $a, b>0$, there is some $b^{\prime} \geq b$ so that $\mathrm{Cl}_{0}\left(Y_{2}(a, b)\right) \subset Y_{2}\left(a / p, b^{\prime}\right)$.

(iii) $\bigcap_{b} Y_{3}(a, b) \subset \bigcap_{a^{\prime}>a} \operatorname{Int}_{0}\left(\bigcap_{b} Y_{3}\left(a^{\prime}, b\right)\right)$ for all $a>0$.

(iv) $Y_{4}(a, b)$ is open in $U_{0}$ for all $a, b>0$.

Let $x \in \mathrm{Cl}_{0}\left(Y_{1}(a, b)\right)$. With the notation of Proposition 8.11, for each $r \geq b$, if $y \in Y_{1}(a, b) \cap V(x, r)$, then

$$
\frac{\log v(x, r)}{\log r}=\frac{\log v(x,\lfloor r\rfloor)}{\log r} \leq \frac{\log v(y,\lfloor r\rfloor)}{\log r}=\frac{\log v(y, r)}{\log r} \leq a
$$

because $\phi_{x, y, r \downarrow}$ is injective by Proposition 8.11 ( $(\mathrm{v})$. Therefore $x \in Y_{1}(a, b)$, confirming Claim 10-(ii).

Let $x \in \mathrm{Cl}_{0}\left(Y_{2}(a, b)\right)$, and let $\Lambda:=\Lambda_{|E|, 2 R C}$ be defined by $(3.3)$. If

$$
r \geq b^{\prime}:=\max \left\{C b, C R, \frac{2 C \log \Lambda}{a}\right\}
$$

and $y \in Y_{2}(a, b) \cap V(x, r)$, then

$$
\begin{aligned}
\frac{\log v(x, r)}{r}=\frac{\log v(x,\lfloor r\rfloor)}{r} \geq \frac{\log (v(y,\lfloor r\rfloor / C) / \Lambda)}{r}=\frac{\log v(y,\lfloor r\rfloor / C)-\log \Lambda}{r} \\
=\frac{\log v(y, r / C)-\log \Lambda}{r} \geq \frac{\log v(y, r / C)}{r}-\frac{a}{2 C} \geq \frac{a}{C}-\frac{a}{2 C}=\frac{a}{2 C},
\end{aligned}
$$

using that $\bar{B}(y,\lfloor r\rfloor / C) \cap \operatorname{im} \phi_{x, y,\lfloor r\rfloor}$ is a $2 R C$-net in $\bar{B}(y,\lfloor r\rfloor / C)$ according to Proposition 8.11 -vil), and $|\bar{B}(z, 2 R C)| \leq \Lambda$ for all $z \in \bar{B}(y, r / C)$ by (3.4). Thus $x \in$ $Y_{2}\left(a / p, b^{\prime}\right)$ for $p=2 C$, which confirms Claim 10 -(ii).

Let $x \in \bigcap_{b} Y_{3}(a, b)$. Given $a^{\prime}>a$, for any choice of $\alpha \in\left(a / a^{\prime}, 1\right)$, let

$$
b \geq \max \left\{C R, C^{\frac{1}{1-\alpha}}, C \Lambda^{\frac{1}{a^{\prime}-a / \alpha}}\right\},
$$

where $\Lambda$ is defined like in the proof of Claim 10 (ii). Then $x \in Y_{3}(a, b)$, which means that there is some $r \geq b$ so that $\log v(x, r) / \log r<a$. If $y \in V(x, C r)$, then

$$
\begin{aligned}
\frac{\log v(y, r / C)}{\log (r / C)}= & \frac{\log v(y,\lfloor r\rfloor / C)}{\log (r / C)} \leq \frac{\log (v(x,\lfloor r\rfloor) \Lambda)}{\log (r / C)}=\frac{\log (v(x, r) \Lambda)}{\log (r / C)} \\
& \leq \frac{\log v(x, r)+\log \Lambda}{\log r-\log C}<\frac{a}{1-\frac{\log C}{\log r}}+\frac{\log \Lambda}{\log r-\log C}<\frac{a}{\alpha}+a^{\prime}-\frac{a}{\alpha}=a^{\prime},
\end{aligned}
$$

like in the proof of Claim 10 (ii). It follows that $V(x, C r) \cap U_{0} \subset Y_{3}\left(a^{\prime}, b\right)$. Since this holds for all $b$ large enough, we get $V(x, C r) \cap U_{0} \subset \bigcap_{b} Y_{3}\left(a^{\prime}, b\right)$ by (9.1), and thus $x \in \operatorname{Int}_{0}\left(\cap_{b} Y_{3}\left(a^{\prime}, b\right)\right)$, showing Claim 10 . (iii).

For any $x \in Y_{4}(a, b)$, there is some integer $r \geq b$ such that $\log v(x, r) / r>a$. So $\log v(y, r) / r>a$ for any $y \in V(x, r)$ since $\phi_{x, y, r}$ is injective (Proposition 8.11-V), giving $V(x, r) \cap U_{0} \subset Y_{4}(a, b)$. Therefore $Y_{4}(a, b)$ is open in $U_{0}$. This confirms Claim 10-(iv). 
For $a \in[0, \infty]$, let

$$
\begin{array}{ll}
Y_{1}(a)=\bigcap_{\alpha>a} \bigcup_{b} Y_{1}(\alpha, b), & Y_{2}(a)=\bigcap_{\alpha<a} \bigcup_{b} Y_{2}(\alpha, b), \\
Y_{3}(a)=\bigcap_{\alpha>a} \bigcap_{b} Y_{3}(\alpha, b), & Y_{4}(a)=\bigcap_{\alpha<a} \bigcap_{b} Y_{4}(\alpha, b) .
\end{array}
$$

It is clear that these are the sets of points $x \in U_{0}$ that respectively satisfy

$$
\begin{array}{cl}
\limsup _{r \rightarrow \infty} \frac{\log v(x, r)}{\log r} \leq a, & \liminf _{r \rightarrow \infty} \frac{\log v(x, r)}{r} \geq a, \\
\liminf _{r \rightarrow \infty} \frac{\log v(x, r)}{\log r} \leq a, & \limsup _{r \rightarrow \infty} \frac{\log v(x, r)}{r} \geq a .
\end{array}
$$

Observe also that

$$
a \leq a^{\prime} \Longrightarrow \begin{cases}Y_{1}(a) \subset Y_{1}\left(a^{\prime}\right), & Y_{2}(a) \supset Y_{2}\left(a^{\prime}\right), \\ Y_{3}(a) \subset Y_{3}\left(a^{\prime}\right), & Y_{4}(a) \supset Y_{4}\left(a^{\prime}\right) .\end{cases}
$$

We get the same sets $Y_{1}(a), Y_{2}(a), Y_{3}(a)$ and $Y_{4}(a)$ above if the condition that $a^{\prime}, b \in \mathbb{Q}$ is added in their definitions. So, by Claim 10-(ii), (iii), (iv), the sets $Y_{1}(a)$, $Y_{3}(a)$ and $Y_{4}(a)$ are Borel in $U_{0}$, and therefore they are Baire subsets of $U$. However the same kind of argument, using Claim 10 (ii), does not apply to $Y_{2}(a)$. Thus consider also the set

$$
Y_{2}^{\prime}(a)=\bigcap_{\alpha<a} \bigcup_{b} \mathrm{Cl}_{0}\left(Y_{2}(a, b)\right),
$$

which are Borel in $U_{0}$ and Baire in $U$. Obviously,

$$
a \leq a^{\prime} \Longrightarrow Y_{2}^{\prime}(a) \supset Y_{2}^{\prime}\left(a^{\prime}\right),
$$

and, by Claim 10 - iii),

$$
Y_{2}^{\prime}(p a) \subset Y_{2}(a) .
$$

The sets $Y_{1}(a)$ and $Y_{3}(a)$ are $\mathcal{G}$-saturated for all $a \in[0, \infty]$ by Remark 4.4 (ii), and we have

$$
\mathcal{G}\left(Y_{2}(q a)\right) \subset Y_{2}(a), \quad \mathcal{G}\left(Y_{4}(q a)\right) \subset Y_{4}(a)
$$

for all $a \in[0, \infty]$ and $q>1$ by Remark 4.4 (iii) and Example 3.7. (i) since $\Lambda_{|E|, 0}=1$ $($ see 3.3$)$. So, by 9.4 ,

$$
\mathcal{G}\left(Y_{2}^{\prime}(p q a)\right) \subset Y_{2}(a)
$$

Claim 11. Each of the sets $Y_{1}(a), \mathcal{G}\left(Y_{2}^{\prime}(a)\right), Y_{3}(a)$ and $\mathcal{G}\left(Y_{4}(a)\right)$ is either residual or meager in $U$.

This assertion is a consequence of Theorem 8.25 because the stated sets are Baire and $\mathcal{G}$-saturated in $U$.

We obviously have

$$
Y_{1}(\infty)=Y_{2}(0)=Y_{3}(\infty)=Y_{4}(0)=U_{0} .
$$

Then let

$$
\begin{aligned}
& a_{1}=\inf \left\{a \in[0, \infty] \mid Y_{1}(a) \text { is residual in } U\right\}, \\
& a_{2}=\sup \left\{a \in[0, \infty] \mid Y_{2}(a) \text { is residual in } U\right\}, \\
& a_{3}=\inf \left\{a \in[0, \infty] \mid Y_{3}(a) \text { is residual in } U\right\}, \\
& a_{4}=\sup \left\{a \in[0, \infty] \mid Y_{4}(a) \text { is residual in } U\right\} .
\end{aligned}
$$


Since the growth type of an infinite connected graph of finite type is at least linear and at most exponential, the sets $Y_{1}(a)$ and $Y_{3}(a)$ are the union of finite orbits in $U_{0}$ for all $0 \leq a<1$, and $Y_{2}(\infty)=Y_{4}(\infty)=\varnothing$. So $a_{1}, a_{3} \geq 1$ by Corollary 8.27 , and $a_{2}, a_{4}<\infty$. By 9.2 , the sets $Y_{1}(a), Y_{2}(a), Y_{3}(a)$ or $Y_{4}(a)$ are residual in $U$ if $a>a_{1}, a<a_{2}, a>a_{3}$ or $a<a_{4}$, respectively. Hence, by (9.7), and because

$$
\begin{array}{llll}
Y_{1}\left(a_{1}\right)=\bigcap_{a_{1}<a<\infty} Y_{1}(a) & \text { if } & a_{1}<\infty, \\
Y_{2}\left(a_{2}\right)=\bigcap_{0<a<a_{2}} Y_{2}(a) & \text { if } & a_{2}>0, \\
Y_{3}\left(a_{3}\right)=\bigcap_{a_{1}<a<\infty} Y_{3}(a) & \text { if } & a_{3}<\infty, \\
Y_{4}\left(a_{4}\right)=\bigcap_{0<a<a_{4}} Y_{4}(a) & \text { if } & a_{4}>0,
\end{array}
$$

where $a$ can be taken in $\mathbb{Q}$, we get that $Y_{1}\left(a_{1}\right), Y_{2}\left(a_{2}\right), Y_{3}\left(a_{3}\right)$ and $Y_{4}\left(a_{4}\right)$ are residual in $U$. On the other hand, by (9.2), 9.3), 9.5), 9.6) and Claim 11, the sets $Y_{1}(a), Y_{2}(a), Y_{3}(a)$ or $Y_{4}(a)$ are meager in $U$ if $a<a_{1}, a>p a_{2}, a<a_{3}$ or $a>a_{4}$, respectively. So the following unions are meager in $U$ because they do not change if $a$ is taken in $\mathbb{Q}$ :

$$
\bigcup_{0 \leq a<a_{1}} Y_{1}(a), \quad \bigcup_{p a_{2}<a<\infty} Y_{2}(a), \quad \bigcup_{0 \leq a<a_{3}} Y_{3}(a), \quad \bigcup_{a_{4}<a<\infty} Y_{4}(a) .
$$

Thus the following sets are residual in $U$ :

$$
\begin{aligned}
& Y_{1}\left(a_{1}\right) \backslash \bigcup_{0 \leq a<a_{1}} Y_{1}(a), \quad Y_{2}\left(a_{2}\right) \backslash \bigcup_{p a_{2}<a<\infty} Y_{2}(a), \\
& Y_{3}\left(a_{3}\right) \backslash \bigcup_{0 \leq a<a_{3} / p^{\prime}} Y_{3}(a), \quad Y_{4}\left(a_{4}\right) \backslash \bigcup_{a_{4}<a<\infty} Y_{4}(a) .
\end{aligned}
$$

These are the sets described by the first group of equalities and inequalities of the statement.

Now, let us prove the last two inequalities of the statement on $U_{0, \mathrm{~d}}$. By Claim 10-(iii),

for all $a \in[0, \infty]$. So

$$
Y_{3}(a)=\bigcap_{a^{\prime}>a} \operatorname{Int}_{0}\left(\bigcap_{b} Y_{3}(a, b)\right)
$$

$$
U_{0} \backslash Y_{3}(a)=U_{0} \backslash \bigcap_{a^{\prime}>a} \operatorname{Int}_{0}\left(\bigcap_{b} Y_{3}(a, b)\right)=\bigcup_{a^{\prime}>a}\left(U_{0} \backslash \operatorname{Int}_{0}\left(\bigcap_{b} Y_{3}(a, b)\right)\right) .
$$

Here, we can take $a^{\prime}$ in $\mathbb{Q}$, and thus this expression is a countable union of closed subsets of $U_{0}$. Moreover we know that $U_{0} \backslash Y_{3}(a)$ is residual in $U_{0}$ for $a<a_{3}$, and therefore there is some $b$ such that $U_{0} \backslash \operatorname{Int}_{0}\left(\cap_{b} Y_{3}(a, b)\right)$ has nonempty interior, obtaining that any $\mathcal{G}$-orbit in $U_{0, \mathrm{~d}}$ meets $U_{0} \backslash Y_{3}(a)$. Hence $U_{0, \mathrm{~d}} \subset U_{0} \backslash Y_{3}(a)$ for all $a<a_{3}$ because $Y_{3}(a)$ is $\mathcal{G}$-saturated, obtaining that $U_{0, \mathrm{~d}} \subset U_{0} \backslash \bigcup_{a<a_{3}} Y_{3}(a)$.

Finally,

$$
U_{0} \backslash Y_{4}(a)=U_{0} \backslash \bigcap_{a^{\prime}<a} \bigcap_{b} Y_{4}\left(a^{\prime}, b\right)=\bigcup_{a^{\prime}<a} \bigcup_{b}\left(U_{0} \backslash Y_{4}\left(a^{\prime}, b\right)\right),
$$

where $a^{\prime}$ and $b$ can be taken in $\mathbb{Q}$. Then, by Claim 10 (iv), this expression is a countable union of closed subsets of $U_{0}$. Furthermore $U_{0} \backslash Y_{4}(a)$ is residual for each $a>a_{4}$, obtaining that $U_{0} \backslash Y_{4}\left(a^{\prime}, b\right)$ has nonempty interior in $U_{0}$ for some $a^{\prime}<a$ and $b$. So any $\mathcal{G}$-orbit in $U_{0, \mathrm{~d}}$ meets $U_{0} \backslash Y_{4}(a)$ for all $a>a_{4}$, and therefore

$$
U_{0, \mathrm{~d}} \subset \mathcal{G}\left(U_{0} \backslash Y_{4}(a)\right) \subset U_{0} \backslash \mathcal{G}\left(Y_{4}\left(a^{\prime}\right)\right) \subset U_{0} \backslash Y_{4}\left(a^{\prime}\right)
$$


if $a^{\prime}>a>a_{4}$ by 9.5 . Thus $U_{0, \mathrm{~d}} \subset U_{0} \backslash \cup_{a>a_{4}} Y_{4}(a)$.

Corollary 9.15. Let $(Z, \mathcal{H}, U, \mathcal{G}, E)$ satisfy Hypothesis 1 and suppose that $\mathcal{G}$ is transitive. Then each of the following sets is either meager or residual in $U$ :

(i) the union of $\mathcal{G}$-orbits in $U_{0}$ with polynomial growth;

(ii) the union of $\mathcal{G}$-orbits in $U_{0}$ with exponential growth;

(iii) the union of $\mathcal{G}$-orbits in $U_{0}$ with quasi-polynomial growth;

(iv) the union of $\mathcal{G}$-orbits in $U_{0}$ with quasi-exponential growth; and

$(v)$ the union of $\mathcal{G}$-orbits in $U_{0}$ with pseudo-quasi-polynomial growth.

Moreover,

(a) if the set (iii) is residual in $U$, then it contains $X_{0, \mathrm{~d}}$; and,

(b) if one of the sets (iv) or (v) is meager in $U$, then it does not meet $U_{0, \mathrm{~d}}$.

REMARK 9.16. The properties used in Corollary 9.15 depend only on the growth type of the $\mathcal{G}$-orbits by Remark 4.4 (i).

\subsection{Amenable orbits}

Like in the above section, since the $\mathcal{G}$-orbits are equi-quasi-lattices in themselves, that they are (equi-) amenable means that they are (equi-) Følner.

TheOrem 9.17. Let $(Z, \mathcal{H}, U, \mathcal{G}, E)$ satisfy Hypothesis 1 . Then the following properties hold:

(i) If $\mathcal{G}$ is transitive and some $\mathcal{G}$-orbit in $U_{0}$ is amenable, then all $\mathcal{G}$-orbits in $U_{0, \mathrm{~d}}$ are equi-amenable.

(ii) If $\mathcal{G}$ is minimal and some $\mathcal{G}$-orbit in $U_{0}$ is amenable, then all $\mathcal{G}$-orbits in $U_{0}$ are jointly amenably symmetric.

Proof. Consider the notation of Proposition 8.11. Suppose that $\mathcal{G}(x)$ is Følner for some $x \in U_{0}$. Let $S_{n}$ be a $\mathrm{F} \varnothing$ lner sequence for $\mathcal{G}(x)$, and let $r \in \mathbb{N}$. For each $n$, let $u_{n} \geq C(r+4 C R)$ be an integer such that

$$
S_{n} \subset \bar{B}\left(x, u_{n} / C-r-4 C R\right) .
$$

For $y \in V\left(x, u_{n}\right)$, let $\phi_{n}$ denote $\phi_{x, y, u_{n}}$, and let $S_{y, n}=\operatorname{Pen}\left(\phi_{n}\left(S_{n}\right), 2 C R\right)$. By 9.8 and Proposition 8.11, (iv),

$$
S_{y, n} \subset \bar{B}\left(y, u_{n} / C-r-2 C R\right) .
$$

Moreover, by Proposition $8.11 \mathrm{v}$,

$$
\left|S_{n}\right| \leq\left|S_{y, n}\right| .
$$

Claim 12. $\partial_{r} S_{y, n} \subset \operatorname{Pen}\left(\phi_{n}\left(\partial_{C(r+4 C R)} S_{n}\right), 2 C R\right)$.

Observe that the right hand side of this inclusion is well defined by $(9.8)$. To prove this inclusion, take first any $z \in \partial_{r} S_{y, n} \backslash S_{y, n}$. Then $d\left(z, \phi_{n}\left(S_{n}\right)\right)>2 C R$ and there is some $z_{0} \in S_{n, y}$ such that $d\left(z, z_{0}\right) \leq r$. Thus there is some $z_{0}^{\prime} \in \phi_{n}\left(S_{n}\right)$ so that $d\left(z_{0}, z_{0}^{\prime}\right) \leq 2 C R$, obtaining $d\left(z, z_{0}^{\prime}\right) \leq 2 C R+r$ by the triangle inequality. By 9.9 ,

$$
d(y, z) \leq d\left(y, z_{0}\right)+d\left(z_{0}, z\right) \leq \frac{u_{n}}{C}-r-2 C R+r=\frac{u_{n}}{C}-2 C R .
$$

By Proposition 8.11-vid), it follows that there is some $z^{\prime} \in \bar{B}\left(y, u_{n} / C\right) \cap \operatorname{im} \phi_{n}$ such that $d\left(z, z^{\prime}\right) \leq 2 C R$. Then $d\left(z_{0}^{\prime}, z^{\prime}\right) \leq 4 C R+r$ by the triangle inequality. We have

$$
d\left(z^{\prime}, \phi_{n}\left(S_{n}\right)\right) \geq d\left(z, \phi_{n}\left(S_{n}\right)\right)-d\left(z^{\prime}, z\right)>2 C R-2 C R=0 ;
$$


i.e., $z^{\prime} \notin \phi_{n}\left(S_{n}\right)$. Let $\bar{z}_{0}^{\prime}=\phi_{n}^{-1}\left(z_{0}^{\prime}\right) \in S_{n}$ and $\bar{z}^{\prime}=\phi_{n}^{-1}\left(z^{\prime}\right) \in \mathcal{G}(x) \backslash S_{n}$. By Proposition $8.11, \mathrm{v}$,

$$
d\left(\bar{z}_{0}^{\prime}, \bar{z}^{\prime}\right) \leq C d\left(z_{0}^{\prime}, z^{\prime}\right) \leq C(4 C R+r) .
$$

Thus

$$
\bar{z}^{\prime} \in \operatorname{Pen}\left(S_{n}, C(4 C R+r)\right) \backslash S_{n} \subset \partial_{C(4 C R+r)} S_{n} .
$$

So $z^{\prime} \in \phi_{n}\left(\partial_{C(4 C R+r)} S_{n}\right)$, and therefore $z \in \operatorname{Pen}\left(\phi_{n}\left(\partial_{C(4 C R+r)} S_{n}\right), 2 C R\right)$.

Now take any $z \in \partial_{r} S_{y, n} \cap S_{y, n}$. Then there are points $z_{0} \in \phi_{n}\left(S_{n}\right)$ and $z_{1} \epsilon$ $\mathcal{G}(y) \backslash S_{y, n}$ such that $d\left(z, z_{0}\right) \leq 2 C R$ and $d\left(z, z_{1}\right) \leq r$, obtaining $d\left(z_{0}, z_{1}\right) \leq 2 C R+$ $r$ by the triangle inequality. We have $d\left(z_{1}, \phi_{n}\left(S_{n}\right)\right)>2 C R$ because $z_{1} \notin S_{y, n}$. By (9.9),

$$
d\left(y, z_{1}\right) \leq d\left(y, z_{0}\right)+d\left(z_{0}, z_{1}\right) \leq \frac{u_{n}}{C}-r-2 C R+2 C R+r=\frac{u_{n}}{C} .
$$

By Proposition 8.11 vil), it follows that there is some $z_{1}^{\prime} \in \bar{B}\left(y, u_{n} / C\right) \cap \operatorname{im} \phi_{n}$ such that $d\left(z_{1}, z_{1}^{\prime}\right) \leq 2 C R$. Then $d\left(z_{0}, z_{1}^{\prime}\right) \leq 4 C R+r$ by the triangle inequality. We have

$$
d\left(z_{1}^{\prime}, \phi_{n}\left(S_{n}\right)\right) \geq d\left(z_{1}, \phi_{n}\left(S_{n}\right)\right)-d\left(z_{1}^{\prime}, z_{1}\right)>2 C R-2 C R=0 ;
$$

i.e., $z_{1}^{\prime} \notin \phi_{n}\left(S_{n}\right)$. Let $\bar{z}_{0}=\phi_{n}^{-1}\left(z_{0}^{\prime}\right) \in S_{n}$ and $\bar{z}_{1}^{\prime}=\phi_{n}^{-1}\left(z_{1}^{\prime}\right) \in \mathcal{G}(x) \backslash S_{n}$. By Proposition $8.11,(\mathrm{v})$,

$$
d\left(\bar{z}_{0}, \bar{z}_{1}^{\prime}\right) \leq C d\left(z_{0}, z_{1}^{\prime}\right) \leq C(4 C R+r) .
$$

Thus

$$
\bar{z}_{0} \in \operatorname{Pen}\left(\mathcal{G}(x) \backslash S_{n}, C(4 C R+r)\right) \cap S_{n} \subset \partial_{C(4 C R+r)} S_{n} .
$$

So $z_{0} \in \phi_{n}\left(\partial_{C(4 C R+r)} S_{n}\right)$, and therefore $z \in \operatorname{Pen}\left(\phi_{n}\left(\partial_{C(4 C R+r)} S_{n}\right), 2 C R\right)$. This completes the proof of Claim 12

By Claim 12 and Proposition 8.11 (v),

$$
\begin{aligned}
&\left|\partial_{r} S_{y, n}\right| \leq\left|\operatorname{Pen}\left(\phi_{n}\left(\partial_{C(r+4 C R)} S_{n}\right), 2 C R\right)\right| \\
& \quad \leq \Lambda_{|E|, 2 R C}\left|\phi_{n}\left(\partial_{C(r+4 C R)} S_{n}\right)\right|=\Lambda_{|E|, 2 R C}\left|\partial_{C(r+4 C R)} S_{n}\right|
\end{aligned}
$$

in particular, by (3.7),

$$
\left|\partial S_{y, n}\right| \leq \Lambda_{|E|, 2 R C}\left|\partial_{C(1+4 C R)} S_{n}\right| \leq \Lambda_{|E|, 2 R C} \Lambda_{|E|, C(1+4 C R)-1}\left|\partial S_{n}\right| .
$$

Assume that $\mathcal{G}$ is transitive. For every dense $\mathcal{G}$-orbit $\mathcal{O}$ and all $n$, there is some $y_{n} \in \mathcal{O} \cap V\left(x, u_{n}\right)$. By $9.10,9.12$ and 3.7 , the sets $S_{y_{n}, n}$ form a Følner sequence in $\mathcal{O}$, and therefore $\mathcal{O}$ is amenable.

Assume that every dense $\mathcal{G}$-orbit is unbounded, for otherwise $\mathcal{G}$ would have only one orbit. Then, for every dense $\mathcal{G}$-orbit $\mathcal{O}$, write

$$
\mathcal{O} \cap V\left(x, u_{n}\right)=\{y(\mathcal{O}, m, n) \mid m \in \mathbb{N}\},
$$

and let $S_{\mathcal{O}, m, n}=S_{y(\mathcal{O}, m, n), n}$.

Suppose that $\mathcal{O} \subset U_{0}$. Given $m, n$ and $t \in \mathbb{N}$, and any $\mathcal{G}$-orbit $\mathcal{O}^{\prime}$ in $U_{0, \mathrm{~d}}$, let $v_{n}=u_{n}+C(2 C R+t)$ and

$$
\mathcal{N}_{\mathcal{O}, \mathcal{O}^{\prime}, m, n, t}=\left\{m^{\prime} \in \mathbb{N} \mid y\left(\mathcal{O}^{\prime}, m^{\prime}, n\right) \in V\left(y(\mathcal{O}, m, n), v_{n}\right) \cap V\left(x, u_{n}\right)\right\},
$$

which is a nonempty set. Take a Følner sequence $X_{n}$ of $\mathcal{O}$ such that $S_{\mathcal{O}, m, n} \subset X_{n} \subset$ $\operatorname{Pen}\left(S_{\mathcal{O}, m, n}, t\right)$. Thus, by 9.9 ,

$$
\begin{aligned}
X_{n} & \subset \bar{B}\left(y(\mathcal{O}, m, n), u_{n} / C-r-2 C R+t\right) \\
& =\bar{B}\left(y(\mathcal{O}, m, n), v_{n} / C-r-4 C R\right) .
\end{aligned}
$$


For the sake of simplicity, given $n$ and any $m^{\prime} \in \mathcal{N}_{\mathcal{O}^{\prime}, m, n}$, write $y=y(\mathcal{O}, m, n)$ and $y^{\prime}=y\left(\mathcal{O}^{\prime}, m^{\prime}, n\right)$. Let

$$
Y_{\mathcal{O}, \mathcal{O}^{\prime}, m^{\prime}, n}=\operatorname{Pen}\left(\phi_{y, y^{\prime}, v_{n}}\left(X_{n}\right), 2 C R\right) .
$$

By Remark 8.12 (ii),

$$
\begin{aligned}
S_{\mathcal{O}^{\prime}, m^{\prime}, n} & =\operatorname{Pen}\left(\phi_{x, y^{\prime}, u_{n}}\left(S_{n}\right), 2 C R\right)=\operatorname{Pen}\left(\phi_{y, y^{\prime}, v_{n}} \circ \phi_{x, y, u_{n}}\left(S_{n}\right), 2 C R\right) \\
& \subset \operatorname{Pen}\left(\phi_{y, y^{\prime}, v_{n}}\left(S_{\mathcal{O}, m, n}\right), 2 C R\right) \subset \operatorname{Pen}\left(\phi_{y, y^{\prime}, v_{n}}\left(X_{n}\right), 2 C R\right)=Y_{\mathcal{O}, \mathcal{O}^{\prime}, m^{\prime}, n},
\end{aligned}
$$

and, by (3.1), Proposition 8.11, (iv) and Remark 8.12,

$$
\begin{gathered}
Y_{\mathcal{O}, \mathcal{O}^{\prime}, m^{\prime}, n}=\operatorname{Pen}\left(\phi_{y, y^{\prime}, v_{n}}\left(X_{n}\right), 2 C R\right) \subset \operatorname{Pen}\left(\phi_{y, y^{\prime}, v_{n}}\left(\operatorname{Pen}\left(S_{\mathcal{O}, m, n}, t\right)\right), 2 C R\right) \\
=\operatorname{Pen}\left(\phi_{y, y^{\prime}, v_{n}}\left(\operatorname{Pen}\left(\phi_{x, y, u_{n}}\left(S_{n}\right), 2 C R+t\right)\right), 2 C R\right) \\
\subset \operatorname{Pen}\left(\phi_{y, y^{\prime}, v_{n}} \circ \phi_{x, y, u_{n}}\left(S_{n}\right), 4 C R+t\right) \\
=\operatorname{Pen}\left(\phi_{x, y^{\prime}, u_{n}}\left(S_{n}\right), 4 C R+t\right)=\operatorname{Pen}\left(S_{\mathcal{O}^{\prime}, m^{\prime}, n}, 2 C R+t\right) .
\end{gathered}
$$

Furthermore, applying 9.10, 9.11 and 9.12 to $X_{n}$ and $Y_{\mathcal{O}, \mathcal{O}^{\prime}, m^{\prime}, n}$, we get

$$
\begin{aligned}
\left|\partial_{r} Y_{\mathcal{O}, \mathcal{O}^{\prime}, m^{\prime}, n}\right| /\left|Y_{\mathcal{O}, \mathcal{O}^{\prime}, m^{\prime}, n}\right| & \leq \Lambda_{|E|, 2 R C}\left|\partial_{C(r+4 C R)} X_{n}\right| /\left|X_{n}\right|, \\
\left|\partial Y_{\mathcal{O}, \mathcal{O}^{\prime}, m^{\prime}, n}\right| /\left|Y_{\mathcal{O}, \mathcal{O}^{\prime}, m^{\prime}, n}\right| & \leq \Lambda_{|E|, 2 R C} \Lambda_{|E|, C(1+4 C R)-1}\left|\partial X_{n}\right| /\left|X_{n}\right| .
\end{aligned}
$$

This shows that the dense $\mathcal{G}$-orbits in $U_{0}$ are equi-amenable.

Next assume that $\mathcal{G}$ is minimal. Then, by Proposition 8.6, for every $\mathcal{O}$ and $n$, there is some $L_{n} \in \mathbb{N}$ so that $\mathcal{O} \cap V\left(x, u_{n}\right)$ is an $L_{n}$-net of $\mathcal{O}$ for any $\mathcal{G}$-orbit $\mathcal{O}$. Then $\cup_{m} S_{\mathcal{O}, m, n}$ is an $\left(L_{n}+u_{n} / C-r-2 C R\right)$-net in $\mathcal{O}$ by $(9.9)$. Similarly, for every $\mathcal{O}$, $m, n$ and $t \in \mathbb{N}$, there is some $L_{\mathcal{O}, m, n, t} \in \mathbb{N}$ so that $\mathcal{O}^{\prime} \cap V\left(y(\mathcal{O}, m, n), v_{n}\right) \cap V\left(x, u_{n}\right)$ is an $L_{\mathcal{O}, m, n, t}$-net of $\mathcal{O}^{\prime}$ for any $\mathcal{G}$-orbit $\mathcal{O}^{\prime}$. Then $\cup_{m^{\prime} \in \mathcal{N}_{\mathcal{O}, \mathcal{O}^{\prime}, m, n, t}} S_{\mathcal{O}^{\prime}, m^{\prime}, n, t}$ is an $\left(L_{\mathcal{O}, m, n, t}+u_{n} / C-r-2 C R\right)$-net in $\mathcal{O}^{\prime}$ by 9.9 . This shows that, when $\mathcal{G}$ is minimal, all $\mathcal{G}$-orbits in $U_{0}$ are jointly amenably symmetric.

\subsection{Asymptotic dimension of the orbits}

For $r \in \mathbb{Z}^{+} \cup\{\infty\}$ and $R, D \in \mathbb{Z}^{+}$and $n \in \mathbb{N}$, let $Y(r, R, D, n)$ be the set of elements $x \in U_{0}$ for which there exists families $\mathcal{V}_{0}, \ldots, \mathcal{V}_{n}$ of subsets of the bal 1 $B(x, r)$ such that:

(a) $\operatorname{diam} V \leq D$ for all $V \in \mathcal{V}_{i}$;

(b) $d\left(V, V^{\prime}\right) \geq R$ if $V \neq V^{\prime}$ in $\mathcal{V}_{i}$; and

(c) $\cup_{i=0}^{n} \mathcal{V}_{i}$ covers $B(x, r)$.

We have

$$
r \geq r^{\prime}, R \geq R^{\prime}, D \leq D^{\prime} \Longrightarrow Y(r, R, D, n) \subset Y\left(r^{\prime}, R^{\prime}, D^{\prime}, n\right) ;
$$

in particular,

$$
Y(R, D, n):=Y(\infty, R, D, n) \subset Y(r, R, D, n)
$$

for all $r \in \mathbb{Z}^{+}$. To see the above inclusion, for a family $\mathcal{V}$ of subsets of $B\left(x, r^{\prime}\right)$, consider the family $\left.\mathcal{V}\right|_{B(x, r)}$ of intersections of the elements of $\mathcal{V}$ with $B(x, r)$. Note that each set $Y(R, D, n)$ is saturated. Moreover, by Proposition 7.14.

$$
\bigcap_{R} \bigcup_{D} Y(R, D, n)=\left\{x \in U_{0} \mid \operatorname{asdim} \mathcal{G}(x) \leq n\right\} .
$$

Lemma 9.18. $Y(R, D, n)=\bigcap_{r} Y(r, R, D, n)$.

\footnotetext{
${ }^{1}$ Recall that $B(x, \infty)=\mathcal{G}(x)$.
} 
Proof. Let $x \in \bigcap_{r} Y(r, R, D, n)$. Construct a graph with vertices the elements $\left(r, \mathcal{V}_{0}, \ldots, \mathcal{V}_{n}\right)$, where $r \in \mathbb{Z}^{+}$and $\mathcal{V}_{0}, \ldots, \mathcal{V}_{n}$ are families of subsets of $B(x, r)$ satisfying a - (c) with these $x$ and $r$, and having an edge from a vertex $\left(r, \mathcal{V}_{0}, \ldots, \mathcal{V}_{n}\right)$ to another vertex $\left(r+1, \mathcal{W}_{0}, \ldots, \mathcal{W}_{n}\right)$ if and only if $\left.\mathcal{W}_{i}\right|_{B(x, r)}=\mathcal{V}_{i}$ for all $i \in\{0, \ldots, n\}$. This graph is locally finite because $B(x, r)$ is finite for all $r$. On the other hand, the fact that $x \in \bigcap_{r} Y(r, R, D, n)$ implies that this graph has arbitrarily large rays. Therefore there is a sequence $r_{k} \uparrow \infty$, and, for each $k$, there are families $\mathcal{V}_{k, 0}, \ldots, \mathcal{V}_{k, n}$ of subsets of $B\left(x, r_{k}\right)$ satisfying (a)-(c) with $x$ and $r_{k}$, and such that, whenever $k<l, \mathcal{V}_{k, i}=\left.\mathcal{V}_{l, i}\right|_{B\left(x, r_{l}\right)}$ for all $i \in\{0, \ldots, n\}$. Let $\mathcal{V}_{i}$ be the family of unions $\bigcup_{k} V_{k}$ for increasing sequences of sets, $V_{0} \subset V_{1} \subset \cdots$, with $V_{k} \in \mathcal{V}_{k, i}$ for all $k$. It is easy to verify that the families $\mathcal{V}_{0}, \ldots, \mathcal{V}_{n}$ satisfy (a) (c) with $x$ and $r=\infty($ on $\mathcal{G}(x))$. Hence $x \in Y(R, D, n)$.

Lemma 9.19. For all $R, D \in \mathbb{Z}^{+}$and $n \in \mathbb{N}$, there is some integer $D^{\prime} \geq D$ so that $\mathrm{Cl}_{0}(Y(R, D, n)) \subset Y\left(R, D^{\prime}, n\right)$.

Proof. With the notation of Proposition 8.11 for any $x \in \mathrm{Cl}_{0}(Y(R, D, n))$, there are points $x_{k} \in Y(R, D, n) \cap V(x, k)$ for all $k \in \mathbb{Z}^{+}$such that $x=\lim _{k} x_{k}$. According to Proposition 8.11 for some $C \in \mathbb{Z}^{+}$, independent of $x$, the maps $\phi_{k}:=\phi_{x, x_{k}, k}: B(x, k) \rightarrow B\left(x_{k}, k\right)$ are non-expanding and equi-bi-Lipschitz with bi-Lipschitz distortion $C$ for all $k$. For each $k$, take families $\mathcal{V}_{k, 0}, \ldots, \mathcal{V}_{k, n}$ of subsets of $B\left(x_{k}, k\right)$ satisfying (a)-(c) with $x_{k}$ and $r=k$. For $k \geq k_{0}$, let

$$
\mathcal{W}_{k, i}=\left\{\phi_{k}^{-1}(V) \mid V \in \mathcal{V}_{k, i}\right\}
$$

for each $i \in\{0, \ldots, n\}$. Obviously, $\bigcup_{i=0}^{n} \mathcal{W}_{k, i}$ covers $B(x, k)$. Let $W=\phi_{k}^{-1}(V) \in \mathcal{W}_{k, i}$ with $V \in \mathcal{V}_{k, i}$. For $w, w^{\prime} \in W$,

$$
d\left(w, w^{\prime}\right) \leq C d\left(\phi_{k}(w), \phi_{k}\left(w^{\prime}\right)\right) \leq C D,
$$

showing that diam $W \leq C D$. Take a different set $W^{\prime}=\phi_{k}^{-1}\left(V^{\prime}\right) \in \mathcal{W}_{k, i}$ for $V^{\prime} \neq V$ in $\mathcal{V}_{k, i}$. For $z \in W$ and $z^{\prime} \in W^{\prime}$,

$$
d\left(z, z^{\prime}\right) \geq d\left(\phi_{k}(z), \phi_{k}\left(z^{\prime}\right)\right) \geq R,
$$

obtaining that $d\left(W, W^{\prime}\right) \geq R$. So $x \in Y(k, R, C D, n)$ for all $k$, and therefore $x \in$ $Y(R, C D, n)$ by Lemma 9.18 . $U_{0}$.

Corollary 9.20. For all $R \in \mathbb{Z}^{+}$and $n \in \mathbb{N}, \cup_{D} Y(D, R, n)$ is an $F_{\sigma}$ subset of

Theorem 9.21. Let $(Z, \mathcal{H}, U, \mathcal{G}, E)$ satisfy Hypothesis 1 . If $\mathcal{G}$ is transitive, then residually many $\mathcal{G}$-orbits have the same asymptotic dimension.

Proof. The key step of the proof is the following assertion.

Claim 13. For each $n \in \mathbb{N}$, the set $\bigcap_{R} \cup_{D} Y(D, R, n)$ is either residual or meager in $U$.

If $\operatorname{Int}_{0}\left(\cup_{D} Y(D, R, n)\right) \neq \varnothing$ for all $R$, then $\cup_{D} Y(D, R, n)$ is residual in $U_{0}$, and therefore it is also residual in $U$, because this set is saturated and $\mathcal{G}$ is transitive. Hence $\bigcap_{R} \cup_{D} Y(D, R, n)$ is also residual in $U$.

If $\operatorname{Int}_{0}\left(\cup_{D} Y\left(D, R_{0}, n\right)\right)=\varnothing$ for some $R_{0} \in \mathbb{Z}^{+}$, then $\bigcup_{D} Y\left(D, R_{0}, n\right)$ is meager in $U_{0}$ by Corollary 9.20 , and therefore it is also meager in $U$, completing the proof of Claim 13 . 
Assume that $\bigcap_{R} \cup_{D} Y(D, R, n)$ is residual in $U$ for some $n \in \mathbb{N}$, and let $n_{0}$ be the least $n$ satisfying this property. By (9.13), $n_{0}$ is the asymptotic dimension of any $\mathcal{G}$-orbit in the $\mathcal{G}$-saturated set

$$
\bigcap_{R} \bigcup_{D} Y\left(D, R, n_{0}\right) \backslash \bigcup_{n=0}^{n_{0}-1} \bigcap_{R} \bigcup_{D} Y(D, R, n)
$$

which is residual by Claim 13 .

Finally, suppose that there is no $n \in \mathbb{N}$ so that $\bigcap_{R} \cup_{D} Y(D, R, n)$ is residual in $U$. Hence $\bigcap_{R} \cup_{D} Y(D, R, n)$ is meager in $U$ for all $n$ by Claim 13 obtaining that

$$
U_{0} \backslash \bigcup_{n=0}^{\infty} \bigcap_{R} \bigcup_{D} Y(D, R, n)
$$

is residual in $U$. Moreover every $\mathcal{G}$-orbit in this $\mathcal{G}$-saturated set is of infinite asymptotic dimension by 9.13 .

\subsection{Highson corona of the orbits}

9.5.1. Limit sets. Let $\mathcal{O}$ be an infinite $\mathcal{G}$-orbit, and $\overline{\mathcal{O}}$ a compactification of $\mathcal{O}$ with corona $\partial \mathcal{O}$.

Definition 9.22. The limit set of any subset $\Sigma \subset \partial \mathcal{O}$, denoted by $\lim _{\Sigma} \mathcal{O}$, is the subset $\cap_{V} \mathrm{Cl}_{U}(V \cap \mathcal{O})$ of $U$, where $V$ runs in the collection of neighborhoods of $\Sigma$ in $\overline{\mathcal{O}}$. If $\Sigma=\{\mathbf{e}\}$, then the notation $\lim _{\mathbf{e}} \mathcal{O}$ is used for $\lim _{\Sigma} \mathcal{O}$.

Take another compactification $\overline{\mathcal{O}}^{\prime} \leq \overline{\mathcal{O}}$ of $\mathcal{O}$ with corona $\partial^{\prime} \mathcal{O}$. Thus there is a continuous extension $\pi: \overline{\mathcal{O}} \rightarrow \overline{\mathcal{O}}^{\prime}$ of id $\operatorname{id}_{\mathcal{O}}$. The restriction $\pi: \partial \mathcal{O} \rightarrow \partial^{\prime} \mathcal{O}$ clearly satisfies $\lim _{\mathbf{e}} \mathcal{O} \subset \lim _{\pi(\mathbf{e})} \mathcal{O}$ for all $\mathbf{e} \in \partial \mathcal{O}$. Thus, roughly speaking, smaller compactifications of the orbits induce larger limit sets.

Each limit set is a closed subset of $U$, which may or may not be $\mathcal{G}$-saturated. The following examples will serve as illustration of this fact.

EXAMPLES 9.23. (i) The corona of the one-point compactification $\mathcal{O}^{*}$ is a singleton, and the corresponding limit set is the standard limit set of the orbit $\mathcal{O}$, which of course $\mathcal{G}$-saturated.

(ii) Consider the compactification of $\mathcal{O}$ whose corona is its coarse end space. The limit set of $\mathcal{O}$ at any of its coarse ends is $\mathcal{G}$-saturated.

(iii) As a particular case of (ii), if $Z=U$ is compact, $\mathcal{H}=\mathcal{G}$ is the pseudogroup generated by a homeomorphism $h$ of $Z$, and $E=\left\{h^{ \pm 1}\right\}$, then $\mathcal{O}$ is isometric to $\mathbb{Z}$, whose coarse end space consists of two points. The corresponding limit sets are the usual $\alpha$ - and $\omega$-limits of $\mathcal{O}$, which are $\mathcal{G}$-saturated.

(iv) Consider the set

$$
\overline{\mathcal{O}}=\mathcal{O} \sqcup \mathrm{Cl}_{Z}(\mathcal{O})=(\mathcal{O} \times\{0\}) \cup\left(\mathrm{Cl}_{Z}(\mathcal{O}) \times\{1\}\right)
$$

with the topology determined as follows: each point of $\mathcal{O} \times\{0\}$ is isolated in $\overline{\mathcal{O}}$, and, a basic neighborhood of a point in $(z, 1) \in \mathrm{Cl}_{Z}(\mathcal{O}) \times\{1\}$ in $\overline{\mathcal{O}}$ is of the form $(V \cap \mathcal{O}) \sqcup V$, where $V$ is any neighborhood of $z$ in $\mathrm{Cl}_{Z}(U)$. Observe that $\overline{\mathcal{O}}$ is a compact Hausdorff space, and $\mathcal{O} \equiv \mathcal{O} \times\{0\}$ is open and dense in $\overline{\mathcal{O}}$; thus $\overline{\mathcal{O}}$ is a compactification of $\mathcal{O}$. In terms of algebras of functions, $\overline{\mathcal{O}}$ corresponds to the algebra of $\mathbb{C}$-valued functions on $\mathcal{O}$ that admit a continuous extension to $\mathrm{Cl}_{Z}(\mathcal{O})$. The corona of this compactification is $\partial \mathcal{O}=\mathrm{Cl}_{Z}(\mathcal{O}) \times\{1\} \equiv \mathrm{Cl}_{Z}(\mathcal{O})$. Moreover, for each $z \in \partial \mathcal{O}$, it is easy to see that $\lim _{z} \mathcal{O}=\{z\}$ if $z \in U$, and $\lim _{z} \mathcal{O}=\varnothing$ if $z \notin U$. Thus $\lim _{z} \mathcal{O}$ may not be $\mathcal{G}$-saturated. 
(v) For the Stone-Čech compactification $\mathcal{O}^{\beta}$, the limit set of $\mathcal{O}$ at any point in its corona $\beta \mathcal{O}$ is either a singleton or empty by (iv) since $\mathcal{O}^{\beta}$ is the maximum of the compactifications.

(vi) For any compactification $\overline{\mathcal{O}} \leq \mathcal{O}^{\nu}$ with corona $\partial \mathcal{O}$, it will be shown that the limit sets of $\mathcal{O}$ at points in $\partial \mathcal{O}$ are $\mathcal{G}$-saturated (Theorem 9.25).

(vii) As a particular case of (vi), if $\left(\mathcal{O}, d_{E}\right)$ is hyperbolic (in the sense of Gromov), we can consider its compactification whose corona is the ideal boundary. Then the limit sets of $\mathcal{O}$ at points in its ideal boundary are $\mathcal{G}$-saturated.

Lemma 9.24. Let $x \in \lim _{\Sigma} \mathcal{O}$ for some $\Sigma \subset \partial \mathcal{O}$. If $\overline{\mathcal{O}} \leq \mathcal{O}^{\nu}, V$ is a neighborhood of $\Sigma$ in $\overline{\mathcal{O}}$, and $S_{1} \subset S_{2} \subset \cdots$ is an increasing sequence of bounded subsets of $V \cap \mathcal{O}$, then there is a sequence $x_{i} \rightarrow x$ in $U$ such that

$$
\bar{B}\left(x_{i}, i\right) \subset V \cap \mathcal{O}, \quad d\left(x_{i},\left\{x_{1}, \ldots, x_{i-1}\right\} \cup S_{i}\right)>3 i .
$$

Proof. Since $\mathcal{O} \leq \mathcal{O}^{\nu}$, there is a continuous extension $\pi: \mathcal{O}^{\nu} \rightarrow \overline{\mathcal{O}}$ of $\operatorname{id}_{\mathcal{O}}$. The sets $\widetilde{V}:=\pi^{-1}(V)$, for neighborhoods $V$ of $\Sigma$ in $\overline{\mathcal{O}}$, form a base of neighborhoods of $\widetilde{\Sigma}:=\pi^{-1}(\Sigma)$ in $\mathcal{O}^{\nu}$, and we have $\widetilde{V} \cap \mathcal{O}=V \cap \mathcal{O}$, obtaining also $\lim _{\widetilde{\Sigma}} \mathcal{O}=\lim _{\Sigma} \mathcal{O}$. So it is enough to consider only the case where $\overline{\mathcal{O}}=\mathcal{O}^{\nu}$.

Assuming $\overline{\mathcal{O}}=\mathcal{O}^{\nu}$, let $W=V \cap \mathcal{O}$, and, for $i \in \mathbb{Z}^{+}$, let

$$
W_{i}=\{y \in W \mid d(y, \mathcal{O} \backslash W)>i\}, \quad V_{i}=\operatorname{Int}_{\mathcal{O}^{\nu}}\left(\mathrm{Cl}_{M^{\nu}}\left(W_{i}\right)\right) .
$$

Observe that $W_{i}=V_{i} \cap \mathcal{O}$ because $\mathcal{O}$ is a discrete space. By Proposition 7.8, $V_{i}$ is a neighborhood of $\Sigma$ in $\mathcal{O}^{\nu}$, and therefore $x \in \mathrm{Cl}_{U}\left(W_{i}\right)$. Then, given a countable base $\left\{P_{i}\right\}$ of open neighborhoods of $x$ in $U$, we have $P_{i} \cap W_{k} \neq \varnothing$ for all $i$ and $k$.

The elements $x_{i}$ are defined by induction on $i$. Let

$$
l_{1}=\max \left\{d(y, \mathcal{O} \backslash W) \mid y \in S_{1}\right\} .
$$

We can choose any element $x_{1} \in P_{1} \cap W_{3+l_{1}}$. Then, for all $y \in S_{1}$,

$$
d\left(x_{1}, y\right) \geq d\left(x_{1}, \mathcal{O} \backslash W\right)-d(y, \mathcal{O} \backslash W)>3+l_{1}-d(y, \mathcal{O} \backslash W) \geq 3 .
$$

Now let $i>1$ and assume that $x_{j}$ is defined for all $j<i$ satisfying $x_{j} \in P_{j} \cap W_{j}$ and $d\left(x_{j},\left\{x_{1}, \ldots, x_{j-1}\right\} \cup S_{j}\right)>3 j$. Let

$$
l_{i}=\max \left\{d(y, \mathcal{O} \backslash W) \mid y \in\left\{x_{1}, \ldots, x_{i-1}\right\} \cup S_{i}\right\},
$$

and choose any point $x_{i} \in P_{i} \cap W_{3 i+l_{i}}$. For all $y \in\left\{x_{1}, \ldots, x_{i-1}\right\} \cup S_{i}$,

$$
d\left(x_{i}, y\right) \geq d\left(x_{i}, \mathcal{O} \backslash W\right)-d(y, \mathcal{O} \backslash W)>3 i+l_{i}-d(y, \mathcal{O} \backslash W) \geq 3 i .
$$

Moreover $\bar{B}\left(x_{i}, i\right) \subset W$ because $x_{i} \in W_{3 i+l_{i}} \subset W_{i}$.

Theorem 9.25. Let $(Z, \mathcal{H}, U, \mathcal{G}, E)$ satisfy Hypothesis 1 , and let $\overline{\mathcal{O}}$ be a compactification of a $\mathcal{G}$-orbit $\mathcal{O}$, with corona $\partial \mathcal{O}$. If $\overline{\mathcal{O}} \leq \mathcal{O}^{\nu}$, then $\lim _{\mathbf{e}} \mathcal{O}$ is $\mathcal{G}$-saturated and nonempty for all $\mathbf{e} \in \partial \mathcal{O}$.

Proof. Let $x \in \lim _{\mathbf{e}} \mathcal{O}$ for some $\mathcal{G}$-orbit $\mathcal{O}$ and $\mathbf{e} \in \partial \mathcal{O}$. Take a sequence $x_{i} \rightarrow x$ satisfying the conditions of Lemma 9.24 with any neighborhood $V$ of e in $\overline{\mathcal{O}}$ and $S_{i}=\varnothing$ for all $i$. Then, by Proposition 8.16 , for any $r \in \mathbb{Z}^{+}$,

$$
\bar{B}(x, r) \subset \bigcap_{i>r} \mathrm{Cl}_{U}\left(\bigcup_{j \geq i} \bar{B}\left(x_{j}, r\right)\right) \subset \mathrm{Cl}_{U}(V \cap \mathcal{O}) .
$$

Since $V$ and $r$ are arbitrary, it follows that $\mathcal{G}(x) \subset \lim _{\mathbf{e}} \mathcal{O}$. Hence $\lim _{\mathbf{e}} \mathcal{O}$ is saturated. 
Let $U_{0}$ be a relatively compact open subset of $U$ that meets all $\mathcal{G}$-orbits. Since, for any open neighborhood $V$ of $\mathbf{e}$ in $\overline{\mathcal{O}}$, the set $V \cap \mathcal{O}$ contains balls of arbitrarily large radius, we have $U_{0} \cap V \neq \varnothing$ by Proposition 8.6, and therefore $\mathrm{Cl}_{U}(V \cap \mathcal{O}) \cap$ $\mathrm{Cl}_{U}\left(U_{0}\right)$ is a nonempty compact set. It follows that

$$
\lim _{\mathbf{e}} \mathcal{O} \cap \mathrm{Cl}_{U}\left(U_{0}\right)=\bigcap_{V} \mathrm{Cl}_{U}(V \cap \mathcal{O}) \cap \mathrm{Cl}_{U}\left(U_{0}\right) \neq \varnothing,
$$

showing that $\lim _{\mathbf{e}} \mathcal{O} \neq \varnothing$.

Definition 9.26. A $\mathcal{G}$-orbit $\mathcal{O}$ is said to be Higson recurrent if $\lim _{\mathbf{e}} \mathcal{O}=U$ for all $\mathbf{e} \in \nu \mathcal{O}$.

REMARK 9.27. Every Higson recurrent $\mathcal{G}$-orbit is obviously dense in $U$.

Theorem 9.28. Let $(Z, \mathcal{H}, U, \mathcal{G}, E)$ satisfy Hypothesis 1 . A $\mathcal{G}$-orbit is Higson recurrent if and only if $\mathcal{G}$ is minimal.

Proof. Let $\mathcal{O}$ be a Higson recurrent $\mathcal{G}$-orbit, and let $Y$ be a $\mathcal{G}$-minimal set (Proposition 8.24). Since $\mathcal{O}$ is dense (Remark 9.27), there is a convergent sequence in $U, x_{i} \rightarrow x$, with $x_{i} \in \mathcal{O}$ and $x \in Y$. Let $P_{1} \supset P_{2} \supset \cdots$ be a nested sequence of open neighborhoods of $Y$ in $U$ such that $\cap_{k} \mathrm{Cl}_{U}\left(P_{k}\right)=Y$. By Proposition 8.16, for each $k \in \mathbb{Z}^{+}$, there is some index $i_{k}$ such that $\bar{B}\left(x_{i_{k}}, k\right) \subset P_{k}$. Hence

$$
\bigcap_{l} \mathrm{Cl}_{U}\left(\bigcup_{k \geq l} \bar{B}\left(x_{i_{k}}, k\right)\right) \subset \bigcap_{l} \mathrm{Cl}_{U}\left(P_{l}\right)=Y .
$$

By Proposition 7.7, $W=\bigcup_{k} B\left(x_{i_{k}}, k\right) \subset \mathcal{O}$ satisfies that $V=\operatorname{Int}_{\mathcal{O}^{\nu}}\left(\mathrm{Cl}_{\mathcal{O}^{\nu}}(W)\right)$ is an open neighborhood of some $\mathbf{e} \in \nu \mathcal{O}$ in $\mathcal{O}^{\nu}$. By Proposition 7.8, the set $V_{l}=$ $V \backslash \bigcup_{k=1}^{l} \bar{B}\left(x_{i_{k}}, k\right)$ is another open neighborhood of $\mathbf{e}$ in $\mathcal{O}^{\nu}$. Since $\mathcal{O}$ is Higson recurrent, we get

$$
U=\lim _{\mathbf{e}} \mathcal{O} \subset \bigcap_{l} \mathrm{Cl}_{U}\left(V_{l} \cap \mathcal{O}\right)=\bigcap_{l} \mathrm{Cl}_{U}\left(\bigcup_{k \geq l} \bar{B}\left(x_{i_{k}}, k\right)\right) \subset Y .
$$

Thus $U$ is the only $\mathcal{G}$-minimal set; i.e., $\mathcal{G}$ is minimal.

Now, assume that $\mathcal{G}$ is minimal. Then $\lim _{\mathbf{e}} \mathcal{O}=U$ for all $\mathcal{G}$-orbit $\mathcal{O}$ and $\mathbf{e} \in \mathcal{O}^{\nu}$ because $\lim _{\mathbf{e}} \mathcal{O}$ is a $\mathcal{G}$-saturated non-empty closed subset of $U$ (Theorem 9.25).

For each $\mathcal{G}$-minimal set $Y$ and any $\mathcal{G}$-orbit $\mathcal{O}$, let $\nu_{Y} \mathcal{O}=\left\{\mathbf{e} \in \nu \mathcal{O} \mid \lim _{\mathbf{e}} \mathcal{O}=Y\right\}$.

TheOREm 9.29. Let $(Z, \mathcal{H}, U, \mathcal{G}, E)$ satisfy Hypothesis 1. For any $\mathcal{G}$-orbit $\mathcal{O}$, the set $\bigcup_{Y} \operatorname{Int}_{\nu \mathcal{O}}\left(\nu_{Y} \mathcal{O}\right)$, where $Y$ runs in the family of $\mathcal{G}$-minimal sets, is dense in $\nu \mathcal{O}$.

Proof. Let $\mathbf{e} \in \nu \mathcal{O}$ for some $\mathcal{G}$-orbit $\mathcal{O}$, and let $V$ be a neighborhood of $\mathbf{e}$ in $\mathcal{O}^{\nu}$. Take another open neighborhood $V^{\prime}$ of $\mathbf{e}$ in $\mathcal{O}^{\nu}$ such that $\mathrm{Cl}_{\mathcal{O}^{\nu}}\left(V^{\prime}\right) \subset V$. By Proposition 8.24 and Theorem $9.25, \lim _{\mathbf{e}} \mathcal{O}$ contains a $\mathcal{G}$-minimal set $Y$. Like in the proof of Theorem 9.28, we can find a subset $W \subset V^{\prime} \cap \mathcal{O}$ so that, for $V^{\prime \prime}=$ $\operatorname{Int}_{\mathcal{O}^{\nu}}\left(\mathrm{Cl}_{\mathcal{O}^{\nu}}(W)\right)$, any $\mathbf{e}^{\prime} \in V^{\prime \prime} \cap \nu \mathcal{O}$ satisfies $\lim _{\mathbf{e}^{\prime}} \mathcal{O} \subset Y$, and therefore $\lim _{\mathbf{e}^{\prime}} \mathcal{O}=Y$ because $Y$ is a minimal set. Thus $V^{\prime \prime} \cap \nu \mathcal{O} \subset \nu_{Y} \mathcal{O}$ and $V^{\prime \prime} \subset \mathrm{Cl}_{\mathcal{O}^{\nu}}\left(V^{\prime}\right) \subset V$.

Suppose that $\left(Z^{\prime}, \mathcal{H}^{\prime}, U^{\prime}, \mathcal{G}^{\prime}, E^{\prime}\right)$ also satisfies Hypothesis 1 and that there is an equivalence $\mathcal{H} \rightarrow \mathcal{H}^{\prime}$, which induces an equivalence $\mathcal{G} \rightarrow \mathcal{G}^{\prime}$, an homeomorphism $U / \mathcal{G} \rightarrow U^{\prime} / \mathcal{G}^{\prime}$, and a bijection between the families of $\mathcal{G}$ - and $\mathcal{G}^{\prime}$-saturated sets. For each $\mathcal{G}$-orbit $\mathcal{O}$, let $\mathcal{O}^{\prime}$ denote the corresponding $\mathcal{G}^{\prime}$-orbit. By Theorem 8.8 , there 
are equi-coarse quasi-isometries of the metric spaces $\left(\mathcal{O}, d_{E}\right)$ to the corresponding metric spaces $\left(\mathcal{O}^{\prime}, d_{E^{\prime}}\right)$. By Propositions 2.26, 2.39 and 7.4, these equi-coarse quasi-isometries induce maps between the corresponding Higson compactifications, $\mathcal{O}^{\nu} \rightarrow \mathcal{O}^{\prime \nu}$, that are continuous at the points of the Higson coronas, and restrict to homeomorphisms between the corresponding Higson coronas, $\nu \mathcal{O} \rightarrow \nu \mathcal{O}^{\prime}$. In fact, the maps $\mathcal{O}^{\nu} \rightarrow \mathcal{O}^{\prime \nu}$ are continuous at all points because the orbits are discrete metric spaces.

Proposition 9.30. For corresponding orbits, $\mathcal{O}$ of $\mathcal{G}$ and $\mathcal{O}^{\prime}$ of $\mathcal{G}^{\prime}$, and corresponding points $\mathbf{e} \in \nu \mathcal{O}$ and $\mathbf{e}^{\prime} \in \nu \mathcal{O}^{\prime}$, the $\mathcal{G}$-saturated set $\lim _{\mathbf{e}} \mathcal{O}$ corresponds to the $\mathcal{G}^{\prime}$-saturated set $\lim _{\mathbf{e}^{\prime}} \mathcal{O}^{\prime}$.

Proof. By Remark 8.3 it is enough to consider the case where $Z^{\prime}=Z, \mathcal{H}^{\prime}=\mathcal{H}$, $\mathrm{Cl}_{Z}(U) \subset U^{\prime}$, and $E^{\prime}=\bar{E}=\{\bar{g} \mid g \in E\}$, where $\bar{g}$ is and extension of each $g \in E$ with $\mathrm{Cl}_{Z}(\operatorname{dom} g) \subset \operatorname{dom} \bar{g}$ (like in Section 8.2). Then $\mathcal{O}=\mathcal{O}^{\prime} \cap U$ and $\mathcal{O}^{\prime}=\mathcal{G}^{\prime}(\mathcal{O})$. Moreover the above coarse quasi-isometry of $\left(\mathcal{O}, d_{E}\right)$ to $\left(\mathcal{O}^{\prime}, d_{\bar{E}}\right)$ is the inclusion map $\mathcal{O} \hookrightarrow \mathcal{O}^{\prime}$, which is a $C$-bi-Lipschitz map whose image is an $R$-net with respect to the metrics $d_{E}$ and $d_{\bar{E}}$ (see Section 8.2. It induces the above continuous map $\mathcal{O}^{\nu} \rightarrow \mathcal{O}^{\prime \nu}$, which is an embedding in this case (Corollary 7.6). Thus we will consider $\mathcal{O}^{\nu}$ as a subspace of $\mathcal{O}^{\prime \nu}$ with $\nu \mathcal{O}=\nu \mathcal{O}^{\prime}$; in particular, $\mathbf{e}=\mathbf{e}^{\prime}$.

Let $V^{\prime}$ be an arbitrary open neighborhood of e in $\mathcal{O}^{\prime \nu}$, and therefore $V=V^{\prime} \cap \mathcal{O}^{\nu}$ is an arbitrary open neighborhood of e in $\mathcal{O}^{\nu}$. We have $V \cap \mathcal{O}=V^{\prime} \cap \mathcal{O}^{\prime} \cap U$. So

$$
\mathrm{Cl}_{U}(V \cap \mathcal{O})=\mathrm{Cl}_{U}\left(V^{\prime} \cap \mathcal{O}^{\prime} \cap U\right)=\mathrm{Cl}_{U^{\prime}}\left(V^{\prime} \cap \mathcal{O}^{\prime}\right) \cap U
$$

where the inclusion " $\supset$ " of last equality holds because $U$ is open in $U^{\prime}$. It follows that

$$
\lim _{\mathbf{e}} \mathcal{O}=\bigcap_{V} \mathrm{Cl}_{U}(V \cap \mathcal{O})=\bigcap_{V^{\prime}} \mathrm{Cl}_{U^{\prime}}\left(V^{\prime} \cap \mathcal{O}^{\prime}\right) \cap U=U \cap \lim _{\mathbf{e}} \mathcal{O}^{\prime}
$$

\subsubsection{Semi weak homogeneity of the Higson corona.}

Definition 9.31. A topological space $X$ is called semi weakly homogeneous if, for all nonempty open subsets $V, V^{\prime} \subset X$, there are homeomorphic nonempty open subsets, $\Omega \subset V$ and $\Omega^{\prime} \subset V^{\prime}$.

TheOREm 9.32. Let $(Z, \mathcal{H}, U, \mathcal{G}, E)$ satisfy Hypothesis 1 . If $\mathcal{G}$ is minimal, then the space $\bigsqcup_{\mathcal{O}} \nu \mathcal{O}$, with $\mathcal{O}$ running in the set of all $\mathcal{G}$-orbits in $U_{0}$, is semi weakly homogeneous.

Proof. Let $\mathcal{O}$ and $\mathcal{O}^{\prime}$ be $\mathcal{G}$-orbits in $U_{0}$, and let e $\in \nu \mathcal{O}$ and $\mathbf{e}^{\prime} \in \nu \mathcal{O}^{\prime}$. Given open neighborhoods, $V$ of $\mathbf{e}$ in $\mathcal{O}^{\nu}$ and $V^{\prime}$ of $\mathbf{e}^{\prime}$ in $\mathcal{O}^{\prime \nu}$, take other open neighborhoods, $V_{0}$ of $\mathbf{e}$ in $\mathcal{O}^{\nu}$ and $V_{0}^{\prime}$ of $\mathbf{e}^{\prime}$ in $\mathcal{O}^{\prime \nu}$, such that $\operatorname{Cl}_{\mathcal{O}^{\nu}}\left(V_{0}\right) \subset V$ and $\mathrm{Cl}_{\mathcal{O}^{\prime \nu}}\left(V_{0}^{\prime}\right) \subset V^{\prime}$. By Corollary 7.9, there is a sequence $x_{k}$ in $\mathcal{O}$ such that $\bar{B}\left(x_{k}, k\right) \subset V_{0}$ and $d\left(x_{l}, x_{k}\right)>3 k$ if $l<k$. We have $\lim _{\mathbf{e}^{\prime}} \mathcal{O}^{\prime}=U$ by Theorem 9.28 . Using Lemma 9.24 by induction on $k$, it follows that there are convergent sequences in $U, x_{k, i}^{\prime} \rightarrow x_{k}$, such that $B\left(x_{k, i}^{\prime}, i\right) \subset V_{0}^{\prime} \cap \mathcal{O}^{\prime}$, and $d\left(x_{l, i}^{\prime}, x_{k, j}^{\prime}\right)>3 i$ if $l<k$ and $j \leq i$, or $l=k$ and $j<i$. With the notation of Proposition 8.11, for all $k \in \mathbb{Z}^{+}$, there is some increasing sequence of indices $i_{k}$ such that $i_{k} \geq k$ and $x_{k, i_{k}}^{\prime} \in V\left(x_{k}, k\right)$.

According to Proposition 8.11 (v), vil), the restrictions

$$
\phi_{k}:=\phi_{x_{k}, x_{i_{k}}^{\prime}, k}: B_{k}:=\phi_{k}^{-1}\left(\bar{B}\left(x_{k, i_{k}}^{\prime}, k / C\right)\right) \rightarrow B_{k}^{\prime}:=\bar{B}\left(x_{k, i_{k}}^{\prime}, k / C\right),
$$


for $k \geq C R$, form a family of equi-coarse equivalences. Moreover $B_{k} \subset \bar{B}\left(x_{k}, k\right)$ and $B_{k}^{\prime} \subset \bar{B}\left(x_{k, i_{k}}^{\prime}, k\right) \subset \bar{B}\left(x_{k, i_{k}}^{\prime}, i_{k}\right)$, obtaining $d\left(B_{k}, B_{l}\right)>k$ and $d\left(B_{k}^{\prime}, B_{l}^{\prime}\right)>i_{k} \geq k$ if $l<k$. Then, by Proposition 2.44, the combination of the maps $\phi_{k}$ is coarse equivalence $\phi: B:=\bigcup_{k \geq k_{0}} B_{k} \rightarrow B^{\prime}:=\bigcup_{k \geq k_{0}} B_{k}^{\prime}$. Thus $\nu \phi: \nu B \rightarrow \nu B^{\prime}$ is a homeomorphism by Proposition 7.4 (iii). We have canonical identities $\nu B \equiv \mathrm{Cl}_{\mathcal{O}^{\nu}}(B) \cap \nu \mathcal{O}$ and $\nu B^{\prime} \equiv \mathrm{Cl}_{\mathcal{O}^{\prime \nu}}\left(B^{\prime}\right) \cap \nu \mathcal{O}^{\prime}$ (Corollary 7.6p. Let $V_{1}=\operatorname{Int}_{\mathcal{O}^{\nu}}\left(\mathrm{Cl}_{\mathcal{O}^{\nu}}(B)\right)$ and $V_{1}^{\prime}=$ $\operatorname{Int}_{\mathcal{O}^{\prime \nu}}\left(\mathrm{Cl}_{\mathcal{O}^{\prime \nu}}\left(B^{\prime}\right)\right)$. The open subsets $\Omega_{1}:=V_{1} \cap \nu \mathcal{O} \subset \nu \mathcal{O}$ and $\Omega_{1}^{\prime}:=V_{1}^{\prime} \cap \nu \mathcal{O}^{\prime} \subset$ $\nu \mathcal{O}^{\prime}$ are nonempty by Proposition 7.7 since $B_{k} \supset \bar{B}\left(x_{k}, k / C\right)$ because $\phi_{k}$ is nonexpanding (Proposition 8.11-(iv)). By Corollary 7.10 , the sets $\Omega_{1}$ and $\Omega_{1}^{\prime}$ are also dense in $\nu B$ and $\nu B^{\prime}$, respectively. Hence $\Omega:=\Omega_{1} \cap(\nu \phi)^{-1}\left(\Omega_{1}^{\prime}\right)$ and $\Omega^{\prime}:=\nu \phi\left(\Omega_{1}\right) \cap \Omega_{1}^{\prime}$ are open dense subsets of $\Omega_{1}$ and $\Omega_{1}^{\prime}$, respectively, and therefore $\Omega$ and $\Omega^{\prime}$ are nonempty and open in $\nu M$. Moreover $\nu \phi$ restricts to a homeomorphism $\Omega \rightarrow \Omega^{\prime}$. Finally,

$$
\mathrm{Cl}_{\nu \mathcal{O}}\left(\Omega_{1}\right)=\mathrm{Cl}_{\mathcal{O}^{\nu}}\left(V_{1}\right) \cap \nu \mathcal{O} \subset \mathrm{Cl}_{\mathcal{O}^{\nu}}(B) \cap \nu \mathcal{O} \subset \mathrm{Cl}_{\mathcal{O}^{\nu}}\left(V_{0}\right) \cap \nu \mathcal{O} \subset V \cap \nu \mathcal{O},
$$

and, similarly, $\mathrm{Cl}_{\nu \mathcal{O}^{\prime}}\left(\Omega_{1}^{\prime}\right) \subset V^{\prime} \cap \nu \mathcal{O}^{\prime}$.

\subsection{Measure theoretic versions}

Let $\mu$ be a Borel measure on $Z$. The measure class $[\mu]$ on $Z$ is the set of Borel measures on $Z$ with the same sets of zero measure as $\mu$, and therefore also the same sets of full measure (the complements of the sets of zero measure). If a measurable $A \subset Z$ is of full measure, then $[\mu]$ is said to be supported in $A$. The product measure class $[\mu] \times[\mu]$ on $Z \times Z$ is the measure class represented by the product measure $\mu \times \mu$.

Let $\mu$ a measure on $Z$. The measure class $[\mu]$ is $\mathcal{H}$-invariant if $\mu(B)=0 \Rightarrow$ $\mu(h(B))=0$, for all $h \in \mathcal{H}$ and every measurable $B \subset \operatorname{dom} h$. An $\mathcal{H}$-invariant measure class $[\mu]$ is said to be ergodic (or $\mathcal{H}$-ergodic) when it consists of ergodic measures; i.e., any $\mathcal{H}$-saturated measurable set is either of zero measure, or of full measure for every measure in $[\mu]$. In this case, $[\mu] \times[\mu]$ is also $\mathcal{H}$-invariant and $\mathcal{H}$-ergodic.

Lemma 9.33. Let $X$ be a topological space, and $A \subset X$ a $G_{\delta}$ subset. Then every Borel subset of $A$ is Borel in $X$.

Proof. It is enough to consider the case of an open subset $B \subset A$, that is, $B=A \cap V$ for some open $V \subset X$. Because $A$ is $G_{\delta}$ in $X$, it can be expressed as $A=\bigcap_{n} U_{n}$, for countable many open sets $U_{n} \subset X$. Therefore $B=\bigcap_{n}\left(U_{n} \cap V\right)$ is a $G_{\delta}$ subset of $X$.

Hypothesis 2. A sextuple $(Z, \mathcal{H}, U, \mathcal{G}, E,[\mu])$ is required to satisfy the following conditions:

- $(Z, \mathcal{H}, U, \mathcal{G}, E)$ satisfy Hypothesis 1 , and

- $[\mu]$ is a $\mathcal{G}$-invariant measure class on $U$ such that $U_{0}$ has full measure. then:

Theorem 9.34. Let $(Z, \mathcal{H}, U, \mathcal{G}, E,[\mu])$ satisfy Hypothesis 包. If $[\mu]$ is $\mathcal{G}$-ergodic,

(i) either $[\mu]$-almost all $\mathcal{G}$-orbits are coarsely quasi-isometric to $[\mu]$-almost all $\mathcal{G}$-orbits;

(ii) or else $[\mu]$-almost all $\mathcal{G}$-orbits are coarsely quasi-isometric to $[\mu]$-almost no $\mathcal{G}$-orbit. 
Proof. Consider the notation of Section 9.1. In particular, $Y$ is a $\mathcal{G} \times \mathcal{G}$ saturated Borel subset of $U_{0} \times U_{0}$ by Lemma 9.1 and therefore it is also a Borel subset of $U \times U$ by Lemma 9.33 . By the $\mathcal{G} \times \mathcal{G}$-ergodicity of the product measure class on $U \times U$, either $Y$ is of full measure, or $Y$ is of zero measure.

Suppose $Y$ is of full measure. By Fubini's theorem, the set $Y_{x}=\left\{y \in U_{0} \mid\right.$ $(x, y) \in Y\}$ is of full measure for almost all $x \in U$ and (i) obtains.

Now assume that $Y$ is of measure zero. It follows from Fubini's theorem that $Y_{x}$ is of zero measure for almost all $x \in U$, and (ii) obtains. then:

TheOREm 9.35. Let $(Z, \mathcal{H}, U, \mathcal{G}, E,[\mu])$ satisfy Hypothesis 2. If $[\mu]$ is $\mathcal{G}$-ergodic,

(i) either $[\mu]$-almost all $\mathcal{G}$-orbits have the same growth type;

(ii) or else the growth type of $[\mu]$-almost all $\mathcal{G}$-orbits are comparable with the growth type of $[\mu]$-almost no $\mathcal{G}$-orbit.

Proof. With the notation of Section 9.2, $Y$ is a $\mathcal{G} \times \mathcal{G}$-saturated Borel subset of $U_{0} \times U_{0}$ by Lemma 9.10, and therefore it is also a Borel subset of $U \times U$ by Lemma 9.33. Like in the proof of Theorem 9.34, it follows that, either $Y$ is of full measure, or $Y$ is of zero measure. Hence, either $Y_{\tau}$ is of full measure, or $Y^{\tau}$ is of zero measure. Then the result follows with the arguments of the proof of Theorem 9.34. using $Y_{\tau}$ to get (i), and $Y^{\tau}$ to get (ii).

TheOREM 9.36. Let $(Z, \mathcal{H}, U, \mathcal{G}, E,[\mu])$ satisfy Hypothesis 2. If $[\mu]$ is $\mathcal{G}$-ergodic, then the equalities and inequalities of Theorem 9.14 hold $[\mu]$-almost everywhere with some $a_{1}, a_{3} \in[1, \infty], a_{2}, a_{4} \in[0, \infty)$ and $p \geq 1$.

Proof. Consider the notation of Theorem 9.14 Its proof shows that the sets $Y(a), \mathcal{G}\left(Y_{2}^{\prime}(a)\right), Y_{3}(a)$ and $\mathcal{G}\left(Y_{4}(a)\right)$ are $\mathcal{G}$-invariant and Borel in $U_{0}$ for all $a \in[0, \infty]$. Thus they are also Borel in $U$ by Lemma 9.33. By ergodicity, each of these sets are either of zero measure or of full measure. Then the result follows easily from the definition of these sets, and using (9.2), (9.3), (9.5) and (9.6) like in the proof of Theorem 9.14.

Corollary 9.37. Let $(Z, \mathcal{H}, U, \mathcal{G}, E,[\mu])$ satisfy Hypothesis 2. If $[\mu]$ is $\mathcal{G}$ ergodic, then any of the sets of Corollary 9.15 is either of zero $[\mu]$-measure or of full $[\mu]$-measure.

TheOREM 9.38. Let $(Z, \mathcal{H}, U, \mathcal{G}, E,[\mu])$ satisfy Hypothesis 2. If $[\mu]$ is $\mathcal{G}$-ergodic, then $[\mu]$-almost all $\mathcal{G}$-orbits have the same asymptotic dimension.

Proof. Consider the notation of Theorem 9.21 By Lemma 9.20, each $\mathcal{G}$ saturated set $\bigcap_{R} \cup_{D} Y(D, R, n)$ Borel. So, by ergodicity, this set is, either of full $[\mu]$-measure, or of zero $[\mu]$-measure.

Suppose that $\bigcap_{R} \cup_{D} Y(D, R, n)$ is of full $[\mu]$-measure for some $n$, and let $n_{0}$ be the least $n$ satisfying this property. Like in the proof of Theorem 9.21, $n_{0}$ is the asymptotic dimension of any $\mathcal{G}$-orbit in the $\mathcal{G}$-saturated set 9.14 , which is of full $[\mu]$-measure.

If $\cap_{R} \cup_{D} Y(D, R, n)$ is of zero $[\mu]$-measure for all $n$, then, like in the proof of Theorem 9.21, the $\mathcal{G}$-saturated set 9.15 is of full $[\mu]$-measure, and consists of orbits with infinite asymptotic dimension. 


\section{CHAPTER 10}

\section{Generic coarse geometry of leaves}

This chapter is devoted to recall preliminaries needed about foliated spaces, fixing the notation, so that the main theorems follow directly from their pseudogroup versions. Introductions to foliated spaces, with many examples, are given in [79, [23. Chapter 11], [24, Part 1] and [51.

\subsection{Foliated spaces}

Let $Z$ be a space and let $U$ be an open set in $\mathbb{R}^{n} \times Z(n \in \mathbb{N})$, with coordinates $(x, z)$. For $m \in \mathbb{N}$, a map $f: U \rightarrow \mathbb{R}^{p}(p \in \mathbb{N})$ is (smooth or differentiable) of class $C^{m}$ if its partial derivatives up to order $m$ with respect to $x$ exist and are continuous on $U$. If $f$ is of class $C^{m}$ for all $m$, then it is called (smooth or differentiable) of class $C^{\infty}$.

Let $Z^{\prime}$ be another space, and let $h: U \rightarrow \mathbb{R}^{p} \times Z^{\prime}$ be a map of the form $h(x, z)=\left(h_{1}(x, z), h_{2}(z)\right)$, for maps $h_{1}: U \rightarrow \mathbb{R}^{p}$ and $h_{2}: \operatorname{pr}_{2}(U) \rightarrow Z^{\prime}$. It will be said that $h$ is of class $C^{m}$ if $h_{1}$ is of class $C^{m}$ and $h_{2}$ is continuous.

For $m \in \mathbb{N} \cup\{\infty\}$ and $n \in \mathbb{N}$, a foliated structure ${ }^{1} \mathcal{F}$ of class $C^{m}$ and dimension $\operatorname{dim} \mathcal{F}=n$ on a space $X$ is defined by a collection $\mathcal{U}=\left\{U_{i}, \phi_{i}\right\}$, where $\left\{U_{i}\right\}$ is an open covering of $X$, and each $\phi_{i}$ is a homeomorphism $U_{i} \rightarrow B_{i} \times Z_{i}$, for a locally compact Polish space $Z_{i}$ and an open ball $B_{i}$ in $\mathbb{R}^{n}$, such that the coordinate changes $\phi_{j} \phi_{i}^{-1}: \phi_{i}\left(U_{i} \cap U_{j}\right) \rightarrow \phi_{j}\left(U_{i} \cap U_{j}\right)$ are locally $C^{m}$ maps of the form

$$
\phi_{j} \phi_{i}^{-1}(x, z)=\left(x_{i j}(x, z), h_{i j}(z)\right) .
$$

Each $\left(U_{i}, \phi_{i}\right)$ is called a foliated chart or flow box, the sets $\phi_{i}^{-1}\left(B_{i} \times\{z\}\right)\left(z \in Z_{i}\right)$ are called plaques (or $\mathcal{U}$-plaques), and the collection $\mathcal{U}$ is called a foliated atlas (of class $C^{m}$ ). Two $C^{m}$ foliated atlases on $X$ define the same $C^{m}$ foliated structure if their union is a $C^{m}$ foliated atlas. If we consider foliated atlases so that the sets $Z_{i}$ are open in some fixed space, then $\mathcal{F}$ can be also described as a maximal foliated atlas of class $C^{m}$. The term foliated space (of class $C^{m}$ ) is used for $X \equiv(X, \mathcal{F})$. If no reference to the class $C^{m}$ is indicated, then it is understood that $X$ is a $C^{0}$ (or topological) foliated space. The restriction of $\mathcal{F}$ to some open subset $U \subset X$ is the foliated structure $\left.\mathcal{F}\right|_{U}$ on $U$ defined by the charts of $\mathcal{F}$ whose domains are contained in $U$.

A map between foliated spaces is called foliated if it maps leaves to leaves. A foliated map between $C^{m}$ foliated spaces is said to be of class $C^{m}$ if its local representations in terms of foliated charts are of class $C^{m}$.

\footnotetext{
${ }^{1}$ The term lamination is also used, specially when $X$ is a subspace of a manifold. The term foliation is used when the spaces $Z_{i}$ are open subsets of some Euclidean space, and therefore $X$ is a manifold. The condition to be of class $C^{m}$ for a foliation $\mathcal{F}$ also requires that the maps $\phi_{j} \phi_{i}^{-1}$ are of class $C^{m}$.
} 
The foliated structure of a space $X$ induces a locally Euclidean topology on $X$, the basic open sets being the plaques of all of its foliated charts, which is finer than the original topology. The connected components of $X$ in this topology are called leaves (or $\mathcal{F}$-leaves). Each leaf becomes a connected manifold of dimension $n$ and of class $C^{m}$ with the differential structure canonically induced by $\mathcal{F}$. The leaf which contains each point $x \in X$ will usually be denoted by $L_{x}$. The leaves of $\mathcal{F}$ form a partition of $X$ that determines the (topological) foliated structure. The corresponding quotient space, called leaf space, is denoted by $X / \mathcal{F}$. It is said that $\mathcal{F}$ is transitive (respectively, minimal) when some leaf is dense (respectively, all leaves are dense) in $X$.

Many concepts of manifold theory readily extend to foliated spaces. In particular, if $\mathcal{F}$ is of class $C^{m}$ with $m \geq 1$, there is a vector bundle $T \mathcal{F}$ over $X$ whose fiber at each point $x \in X$ is the tangent space $T_{x} L_{x}$. Observe that $T \mathcal{F}$ is a foliated space of class $C^{m-1}$ with leaves $T L$ for leaves $L$ of $X$, and any section of $T F$ is foliated. The same applies to any bundle naturally associated to $T \mathcal{F}$. Then we can consider a $C^{m-1}$ Riemannian structur $2^{2}$ on $T \mathcal{F}$, which will be called a (leafwise) Riemannian metric on $X$. A $C^{m}$ foliated space with a $C^{m-1}$ Riemannian metric is called a $C^{m}$ Riemannian foliated space; the reference to $C^{m}$ is omitted if $m=\infty$.

From now on, it will be assumed that $X$ is locally compact and Polish; i.e., the spaces $Z_{i}$ are locally compact and Polish.

Definition 10.1 (See [63, [23, [52]). A foliated atlas $\mathcal{U}=\left\{U_{i}, \phi_{i}\right\}$ of $\mathcal{F}$ is called regular if:

(i) for all $i$, there is a foliated chart $\left(\widetilde{U}_{i}, \tilde{\phi}_{i}\right)$ of $\mathcal{F}$ so that $\overline{U_{i}} \subset \widetilde{U}_{i}$ and $\left.\tilde{\phi}_{i}\right|_{U_{i}}=\phi_{i}$;

(ii) the cover $\left\{U_{i}\right\}$ of $X$ is locally finite; and,

(iii) for all $i$ and $j$, the closure of every plaque of $\left(U_{i}, \phi_{i}\right)$ meets at most the closure of one plaque of $\left(U_{j}, \phi_{j}\right)$.

Since $X$ is Polish and locally compact, every foliated atlas $\mathcal{U}=\left\{U_{i}, \phi_{i}\right\}$ of $(X, \mathcal{F})$ is refined by a regular atlas $\mathcal{V}=\left\{V_{\alpha}, \psi_{\alpha}\right\}$ in the sense that, for each $\alpha$, there is some index $i(\alpha)$ so that $\overline{V_{\alpha}} \subset U_{i(\alpha)}$ and $\phi_{i(\alpha)}$ extends $\psi_{\alpha}$.

Let $\mathcal{U}=\left\{U_{i}, \phi_{i}\right\}$ be a regular foliated foliated atlas of $\mathcal{F}$ with $\phi_{i}: U_{i} \rightarrow B_{i} \times Z_{i}$, and let $p_{i}: U_{i} \rightarrow Z_{i}$ denote the composition of $\phi_{i}$ with the second factor projection $B_{i} \times Z_{i} \rightarrow Z_{i}$. Then the form 10.1 of the changes of coordinates holds globally, with $x_{i j}: Z_{i j}=\phi_{j}\left(U_{i} \cap U_{j}\right) \rightarrow \mathbb{R}^{n}$ and $h_{i j}: Z_{i j} \rightarrow Z_{j i}$. Each map $h_{i j}$ is determined by the condition $p_{j}=h_{i j} p_{j}$ on $U_{i} \cap U_{j}$. They satisfy the cocycle property $h_{j k} h_{i j}=h_{i k}$ on $U_{i} \cap U_{j} \cap U_{k}$ for all $i, j$ and $k$. The family $\left\{U_{i}, p_{i}, h_{i j}\right\}$ is called a defining cocycle of $\mathcal{F}$ [58, [59].

The maps $h_{i j}$ generate a pseudogroup $\mathcal{H}$ of local transformations of $Z=\sqcup_{i} Z_{i}$, which is called the representative of the holonomy pseudogroup of $\mathcal{F}$ induced by $\mathcal{U}$ (or by $\left\{U_{i}, p_{i}, h_{i j}\right\}$ ). This $\mathcal{H}$ is independent of $\mathcal{U}$ up to pseudogroup equivalences. Let $E=\left\{h_{i j}\right\}$, which is a symmetric family of generators of $\mathcal{H}$. There is a canonical homemorphism between the leaf space and the orbit space, $X / \mathcal{F} \rightarrow Z / \mathcal{H}$, given by $L \mapsto \mathcal{H}\left(p_{i}(x)\right)$ if $x \in L \cap U_{i}$.

By fixing any $b_{i} \in B_{i}$, each $Z_{i}$ can be considered as a subset of $X$, called a local transversal, via

$$
Z_{i} \equiv\left\{b_{i}\right\} \times Z_{i} \subset B_{i} \times Z_{i} \stackrel{\phi_{i}^{-1}}{\longrightarrow} U_{i} .
$$

\footnotetext{
${ }^{2}$ This means a section of the associated bundle over $X$ of positive definite symmetric bilinear forms on the fibers of $T \mathcal{F}$, which is $C^{m-1}$ as foliated map.
} 
It can be assumed that all of these local transversals are mutually disjoint, and thus $Z$ becomes embedded in $X$; then it is called a complete transversal in the sense that it meets all leaves and is locally given by local transversals. Each $\mathcal{H}$-orbit injects into the corresponding $\mathcal{F}$-leaf in this way.

The holonomy groups of the leaves are the germ groups of the corresponding orbits. The leaves with trivial holonomy groups are called leaves without holonomy, and they correspond to orbits with trivial germ groups. Then the union $X_{0}$ of leaves with trivial holonomy groups is a dense $G_{\delta}$ saturated subset of $X$, hence Borel and residual (by Theorem 8.26, or see directly 61 and [42]). When $\mathcal{F}$ is transitive, the union $X_{0, \mathrm{~d}}$ of dense leaves with trivial holonomy groups is a residual subset of $X$ (by Corollary 8.27). If $X=X_{0}$, then it is said that $X$ is a foliated space without holonomy.

By the regularity of $\mathcal{U}$, we can consider the foliated atlas $\widetilde{\mathcal{U}}=\left\{\widetilde{U}_{i}, \tilde{\phi}_{i}\right\}$ given by Definition 10.1 (- (i), where $\tilde{\phi}_{i}: \widetilde{U}_{i} \rightarrow \widetilde{B}_{i} \times \widetilde{Z}_{i}$. By refining $\widetilde{\mathcal{U}}$ if necessary, we can assume that it is also regular. Thus it also induces a representative $\widetilde{\mathcal{H}}$ of the holonomy pseudogroup on $\widetilde{Z}=\sqcup_{i} \widetilde{Z}_{i}$, a symmetric set of generators $\widetilde{E}=\left\{\tilde{h}_{i j}\right\}$ given by (10.1), and a defining cocycle $\left\{\widetilde{U}_{i}, \tilde{p}_{i}, \tilde{h}_{i j}\right\}$. Observe that $Z$ is an open subset of $\widetilde{Z}$ that meets all $\widetilde{\mathcal{H}}$-orbits, $\left.\widetilde{\mathcal{H}}\right|_{Z}=\mathcal{H}$, each $\tilde{h}_{i j}$ extends $h_{i j}$, and $\overline{\operatorname{dom} h_{i j}} \subset \operatorname{dom} \tilde{h}_{i j}$.

Let $\left(X^{\prime}, \mathcal{F}^{\prime}\right)$ be another locally compact Polish foliated space of class $C^{m}$ and dimension $n^{\prime}$. Then $\mathcal{F} \times \mathcal{F}^{\prime}$ denotes the foliated structure on $X \times X^{\prime}$ with leaves $L \times L^{\prime}$, where $L$ and $L^{\prime}$ are leaves of $\mathcal{F}$ and $\mathcal{F}^{\prime}$, respectively. Let $\mathcal{U}^{\prime}=\left\{U_{\alpha}^{\prime}, \phi_{\alpha}^{\prime}\right\}$ be a foliated atlas of $\mathcal{F}^{\prime}$, where $\phi_{\alpha}^{\prime}: U_{\alpha}^{\prime} \rightarrow B_{\alpha}^{\prime} \times Z_{\alpha}^{\prime}$. For all foliated charts $\left(U_{i}, \phi_{i}\right) \in \mathcal{U}$ and $\left(U_{\alpha}^{\prime}, \phi_{\alpha}^{\prime}\right) \in \mathcal{U}^{\prime}$, we get a foliated chart $\left(U_{i} \times U_{\alpha}^{\prime}, \psi_{i \alpha}\right)$ of $\mathcal{F} \times \mathcal{F}^{\prime}$, where $\psi_{i \alpha}$ is the composite

$$
U_{i} \times U_{\alpha}^{\prime} \stackrel{\phi_{i} \times \phi_{\alpha}^{\prime}}{\longrightarrow} B_{i} \times Z_{i} \times B_{\alpha}^{\prime} \times Z_{\alpha}^{\prime} \stackrel{\xi_{i \alpha}}{\longrightarrow} B_{i \alpha} \times Z_{i} \times Z_{\alpha}^{\prime},
$$

where $B_{i \alpha}$ is an open ball in $\mathbb{R}^{n+n^{\prime}}$ and

$$
\xi_{i \alpha}\left(x_{i}, y_{i}, x_{j}, y_{j}\right)=\left(\zeta_{i \alpha}\left(x_{i}, x_{j}\right), y_{i}, y_{j}\right)
$$

for some homeomorphism $\zeta_{i \alpha}: B_{i} \times B_{\alpha}^{\prime} \rightarrow B_{i \alpha}$. The collection $\mathcal{V}=\left\{U_{i} \times U_{\alpha}^{\prime}, \psi_{i \alpha}\right\}$ is a foliated atlas of $\mathcal{F} \times \mathcal{F}^{\prime}$. We can assume that the maps $\zeta_{i \alpha}$ are $C^{m}$ diffeomorphisms, and therefore $\mathcal{F} \times \mathcal{F}^{\prime}$ becomes of class $C^{m}$ with $\mathcal{V}$.

Suppose that $\mathcal{U}^{\prime}$ is regular; in particular, it satisfies Definition 10.1 (ii) with charts $\left(\widetilde{U}_{\alpha}^{\prime}, \tilde{\phi}_{\alpha}^{\prime}\right)$, where $\tilde{\phi}_{\alpha}^{\prime}: \widetilde{U}_{\alpha}^{\prime} \rightarrow \widetilde{B}_{\alpha}^{\prime} \times \widetilde{Z}_{\alpha}^{\prime}$. We can assume that each $\zeta_{i \alpha}$ extends to a homeomorphism $\tilde{\zeta}_{i \alpha}: \widetilde{B}_{i} \times \widetilde{B}_{\alpha}^{\prime} \rightarrow \widetilde{B}_{i \alpha}$ for some open balls $\widetilde{B}_{i \alpha}$ containing $\overline{B_{i \alpha}}$. As before, using $\tilde{\phi}_{i}, \tilde{\phi}_{\alpha}^{\prime}$ and $\tilde{\zeta}_{i \alpha}$, we get a foliated chart $\left(\widetilde{U}_{i} \times \widetilde{U}_{\alpha}^{\prime}, \tilde{\psi}_{i \alpha}\right)$ of $\mathcal{F} \times \mathcal{F}^{\prime}$, which extends $\psi_{i \alpha}$. This shows that $\mathcal{V}$ satisfies Definition 10.1.(i). The other conditions of Definition 10.1 are obviously satisfied, and therefore $\mathcal{V}$ is regular. Observe that, if $\mathcal{H}^{\prime}$ is the representative of the holonomy pseudogroup of $\mathcal{F}^{\prime}$ induced by $\mathcal{U}^{\prime}$, then $\mathcal{H} \times \mathcal{H}^{\prime}$ is the representative of the holonomy pseudogroup of $\mathcal{F} \times \mathcal{F}^{\prime}$ induced by $\mathcal{V}$.

\subsection{Saturated sets}

Consider the notation of Section 10.1. A subset of $X$ is called saturated (or $\mathcal{F}$-saturated) if it is a union of leaves. The saturation (or $\mathcal{F}$-saturation) of a subset $A \subset X$ is the union $\mathcal{F}(A)$ of leaves that meet $A$. The canonical homeomorphism $X / \mathcal{F} \approx Z / \mathcal{H}$ gives a canonical bijection between the families of $\mathcal{F}$-saturated subsets of $X$ and $\mathcal{H}$-saturated subsets of $Z$, which preserves the properties of being open, closed, $G_{\delta}, F_{\sigma}$, Borel, Baire, dense, residual or meager. 
Remark 10.2. Note that each leaf is an $F_{\sigma}$ subset of $X$. Moreover the relation set $R_{\mathcal{F}} \subset X \times X$ of the equivalence relation "being in the same $\mathcal{F}$-leaf" is an $F_{\sigma}$ subset, which follows from Remark 8.21 since

$$
R_{\mathcal{F}}=\bigcup_{i, j}\left(p_{i} \times p_{j}\right)^{-1}\left(R_{\mathcal{H}} \cap\left(Z_{i} \times Z_{j}\right)\right),
$$

where each $p_{i} \times p_{j}$ is a trivial fiber bundle with $\sigma$-compact fibers.

The relation between saturations in $X$ and $Z$ is the following:

$$
\mathcal{F}(A)=\bigcup_{i, j} p_{j}^{-1}\left(\mathcal{H}\left(p_{i}\left(A \cap U_{i}\right)\right) \cap Z_{j}\right)
$$

for any $A \subset X$. Thus $\mathcal{F}(A)$ is open if $A$ is open, which is well known. However the behavior of the saturation is worse in foliated spaces than in pseudogroups: the saturation of a meager, Borel or Baire set may not be meager, Borel or Baire, respectively. But we have the following result.

Lemma 10.3. Let $A \subset V \subset X$, where $V$ is open in $X$. The following properties hold:

(i) If $A$ is residual in $V$, then $\mathcal{F}(A)$ is residual in $\mathcal{F}(V)$.

(ii) If $A$ is meager and $\left.\mathcal{F}\right|_{V}$-saturated in $V$, then $\mathcal{F}(A)$ is meager in $\mathcal{F}(V)$.

Proof. Property (i) follows from 10.2, Lemma 8.19 and Theorem 8.18 (applied to the trivial fiber bundles $p_{i}$ ).

To prove (ii), we can assume that $V$ is some $U_{i}$. In this case, we have the following simplification of 10.2 :

$$
\mathcal{F}(A)=\bigcup_{j} p_{j}^{-1}\left(\mathcal{H}\left(p_{i}(A)\right) \cap Z_{j}\right) .
$$

By Theorem 8.18, $p_{i}(A)$ is meager in $Z_{i}$. So $\mathcal{H}\left(p_{i}(A)\right)$ is meager in $Z$ by Lemma 8.20 Therefore each $p_{j}^{-1}\left(\mathcal{H}\left(p_{i}(A)\right) \cap Z_{j}\right)$ is meager in $U_{j}$ by Theorem 8.18, obtaining that $\mathcal{F}(A)$ is meager.

\subsection{Coarse quasi-isometry type of the leaves}

Consider the notation of Sections 10.1 and 10.2, and suppose from now on that $X$ is compact ${ }^{3}$ Let $R_{\mathcal{F}} \subset X \times X$ be the subset of pairs of points in the same leaf (the relation set of the partition into leaves). For $(x, y) \in R_{\mathcal{F}}$, let $d_{\mathcal{U}}(x, y)$ be the minimum number of $\mathcal{U}$-plaques whose union is connected and contains $\{x, y\}$. This defines a man ${ }^{4} d_{\mathcal{U}}: R_{\mathcal{F}} \rightarrow[0, \infty)$, which is upper semi-continuous, symmetric and satisfies the triangle inequality, but it is not a metri $\left(^{5}\right.$ on the leaves because $d_{\mathcal{U}} \geq 1$. To solve this problem, consider the function $d_{\mathcal{U}}^{*}: R_{\mathcal{F}} \rightarrow[0, \infty)$ given by

$$
d_{\mathcal{U}}^{*}(x, y)= \begin{cases}d_{\mathcal{U}}(x, y) & \text { if } x \neq y \\ 0 & \text { if } x=y\end{cases}
$$

The map $d_{\mathcal{U}}^{*}$ is symmetric, satisfies the triangle inequality and its zero set is the diagonal $\Delta_{X} \subset R_{\mathcal{F}}$, and therefore its restriction to each leaf is a metric. However $d_{\mathcal{U}}^{*}$ is upper semi-continuous only on $R_{\mathcal{F}} \backslash \Delta_{X}$. For each $x \in X, S \subset L_{x}$ and $r \geq 0$,

\footnotetext{
${ }^{3}$ Several concepts of this section do not need compactness of $X$ to be defined.

${ }^{4}$ The same definition gives a map $d_{\mathcal{U}}: X \times X \rightarrow[0, \infty]$ with the analogous properties so that $R_{\mathcal{F}}$ is the set with finite values.

${ }^{5}$ This $d_{\mathcal{U}}$ is a coarse metric on the leaves in the sense of Hurder $6 \mathbf{6 8}$.
} 
the notation $B_{\mathcal{U}}(x, r), \bar{B}_{\mathcal{U}}(x, r)$ and $\operatorname{Pen}_{\mathcal{U}}(S, r)$ will be used for the corresponding open and closed balls, and penumbra in $\left(L_{x}, d_{\mathcal{U}}^{*}\right)$.

A plaque chain (or $\mathcal{U}$-plaque chain) is a finite sequence of $\mathcal{U}$-plaques such that each pair of consecutive plaques has nonempty intersection. For $(x, y) \in R_{\mathcal{F}}$, the value $d_{\mathcal{U}}(x, y)$ equals the least $k \in \mathbb{Z}^{+}$such that there is a plaque chain ${ }^{6}\left(P_{1}, \ldots, P_{k}\right)$ with $x \in P_{1}$ and $y \in P_{2}$.

If $X$ is $C^{1}$, we can also pick up any Riemannian metric $g$ on $X$ and take the corresponding Riemannian distance $d_{g}$ on the leaves, which defines an upper semicontinuous map $d_{g}: R_{\mathcal{F}} \rightarrow[0, \infty)$.

Since $X$ is compact, $\mathcal{U}$ is finite by Definition 10.1 -(iii). Moreover $\overline{U_{i}}$ is compact, and therefore every $Z_{i}$ has compact closure in $\widetilde{Z}_{i}$, So $Z$ has compact closure in $\widetilde{Z}$. By the observations of Section 10.1, it follows that $\widetilde{\mathcal{H}}$ is compactly generated and $E$ is a symmetric system of compact generation of $\widetilde{\mathcal{H}}$ on $Z$.

Lemma 10.4. E is recurrent.

Proof. There is an open cover $\left\{V_{i}\right\}$ of $X$ such that $\overline{V_{i}} \subset U_{i}$ for all $i$. Then $W_{i}=p_{i}\left(V_{i}\right)$ is open with compact closure in $Z_{i}$. Thus $W=\cup_{i} W_{i}$ is open with compact closure in $Z$.

Take any $z \in Z$, which is in some $Z_{i}$. Then there is some $x \in U_{i}$ such that $p_{i}(x)=z$. Since $\left\{V_{j}\right\}$ covers $X$, there is also some $V_{j}$ containing $x$. So $h_{i j}(z)=$ $p_{j}(x) \in W_{j}$. This shows that $\mathcal{H}(z) \cap W$ is a 1 -net in $\mathcal{H}(z)$ with $d_{E}$.

By Lemma 10.4 and Theorem 8.8, $Z, \mathcal{H}$ and $E$ satisfy the conditions to determine a coarse quasi-isometry type on the orbits that is "equi-invariant" by pseudogroup equivalences.

The following result is well known, at least in the case of foliations of manifolds. For the reader's convenience, its proof is indicated by its relevance in this work, and because some subtleties show up in the case of foliated spaces.

Proposition 10.5. The following properties hold:

(i) For any other regular foliated atlases $\mathcal{V}$ of $\mathcal{F}, d_{\mathcal{U}}^{*}$ and $d_{\mathcal{V}}^{*}$ are equi-Lipschitz equivalent on the leaves.

(ii) Suppose that $\mathcal{F}$ is $C^{3}$. Then, for any $C^{2}$ Riemannian metric $g$ on $X, d_{\mathcal{U}}^{*}$ and $d_{g}$ are equi-large scale Lipschitz equivalent on the leaves.

(iii) The leaves with $d_{\mathcal{U}}^{*}$ are equi-coarsely quasi-isometric to the corresponding $\mathcal{H}$ orbits with $d_{E}$.

Proof. Let $R_{i} \subset R_{\mathcal{F}}$ be the relation set defined by the restriction $\left.\mathcal{F}\right|_{U_{i}}$. Note that $\bigcup_{i} R_{i}$ is an open neighborhood of $\Delta_{X}$ in $R_{\mathcal{F}}$. By Definition 10.1. (ii), (iii) and the compactness of $X$, it follows that each $R_{i}$ has compact closure in $R_{\mathcal{F}}$. Hence, by the upper semicontinuity of $d_{\mathcal{V}}$ and since $\mathcal{U}$ is finite, there is some $C>0$ such that $\sup d_{\mathcal{V}}^{*}\left(R_{i}\right) \leq \sup d_{\mathcal{V}}\left(R_{i}\right) \leq C$ for all $i$. This means that the $d_{\mathcal{V}}^{*}$-diameter of all plaques of $\mathcal{U}$ is $\leq C$, obtaining that $d_{\mathcal{V}}^{*} \leq C d_{\mathcal{U}}^{*}$ on $R_{\mathcal{F}}$. This proves (i).

\footnotetext{
${ }^{6}$ For a leaf $L$, and plaques $P, Q \subset L$ (respectively, $x, y \in L$ ), let $\bar{d}_{\mathcal{U}}(P, Q)$ (respectively, $\left.\bar{d}_{\mathcal{U}}(x, y)\right)$ be the least $k \in \mathbb{N}$ such that there is a plaque chain $\left(P_{0}, \ldots, P_{k}\right)$ with $P_{0}=P$ and $P_{k}=Q$. This defines a metric $\bar{d}_{\mathcal{U}}$ on the set of plaques in $L$, which can be identified to the metric $d_{E}$ on the corresponding $\mathcal{H}$-orbit $\mathcal{O}$, where plaques in $L$ are identified to the points in $\mathcal{O}$ via the maps $p_{i}$. However the map $\bar{d}_{\mathcal{U}}=d_{\mathcal{U}}-1: L \times L \rightarrow[0, \infty)$, defined in this way, does not satisfy the triangle inequality.
} 
Similarly, by the upper semicontinuity of $d_{g}$, we get $\sup d_{g}\left(R_{i}\right) \leq K$ for some $K>0$, obtaining ${ }^{7} d_{g} \leq K d_{\mathcal{U}}^{*}$ on $R_{\mathcal{F}}$.

Consider the disjoint union of the leaves as a Riemannian manifold with $g$.

Claim 14. The disjoint union of the leaves has a positive injectivity radius.

For each $i$, take relatively compact open subsets, $Z_{i}^{\prime} \subset Z_{i}$ and $B_{i}^{\prime} \subset B_{i}$ such that the sets $U_{i}^{\prime}=\phi^{-1}\left(B_{i}^{\prime} \times Z_{i}^{\prime}\right)$ cover $X$. Since $\mathcal{U}$ is finite, it is enough to prove that, for all $i$, there is some $C_{i}>0$ such that the injectivity radius of $L_{x}$ at every $x \in U_{i}^{\prime}$ is $\operatorname{inj}_{g}(x) \geq C_{i}$. Let $\psi: \widetilde{B}_{i} \rightarrow N$ be a $C^{3}$ open embedding into a closed $n$-manifold, and set $V=\psi\left(\widetilde{B}_{i}\right)$ and $W=N \backslash \psi\left(\overline{B_{i}}\right)$. Let $\{\lambda, \mu\}$ be a $C^{3}$ partition of unity of $N$ subordinated to its open covering $\{V, W\}$. For all $z \in \overline{Z_{i}^{\prime}}$, let $g_{z}$ be the Riemannian metric on $V$ that corresponds to $g$ by the $C^{3}$ diffeomorphism

$$
\tilde{\phi}_{i}^{-1}\left(\{z\} \times \widetilde{B}_{i}\right) \stackrel{\tilde{\phi}_{i}}{\longrightarrow}\{z\} \times \widetilde{B}_{i} \equiv \widetilde{B}_{i} \stackrel{\psi}{\longrightarrow} V .
$$

Pick up any $C^{2}$ Riemannian metric $h$ on $N$. Then the metrics $h_{z}=\lambda g_{z}+\mu h\left(z \in \overline{Z_{i}^{\prime}}\right)$ form o compact family of $C^{2}$ Riemannian metrics on $N$ with the $C^{2}$ topology. By the continuous dependence of the injectivity radius on the Riemannian metric with respect to the $C^{2}$ topology on closed manifolds [40], 87], there is some $K>0$ such that $\operatorname{inj}_{h_{x}}(y) \geq K$ for all $y \in N$. Let $K^{\prime}>0$ denote the infimum of the $h_{z}$-distance between $\psi\left(B_{i}^{\prime}\right)$ and $W$, with $z$ running in $\overline{Z_{i}^{\prime}}$, and set $C_{i}=\min \left\{K, K^{\prime}\right\}>0$. Since $h_{x}=g_{x}$ on $\psi\left(B_{i}\right)$, we get $\operatorname{inj}_{g}(x) \geq C_{i}$ for all $x \in U_{i}^{\prime}$. This completes the proof of Claim 14.

By Claim 14, the continuous dependence of the geodesic flow on the metric with respect to the $C^{1}$ topology [87, Lemma 1.5], and because $X$ is compact, it easily follows that there is some $\varepsilon>0$ such that

$$
d_{g}^{-1}([0, \varepsilon)) \subset \bigcup_{i} R_{i} .
$$

Take points $x$ and $y$ in a leaf $L$. Suppose first that $d_{g}(x, y) \geq \varepsilon / 2$, and let $\gamma(t)$ $(0 \leq t \leq 1)$ be a minimizing geodesic in $L$ with $\gamma(0)=x$ and $\gamma(1)=y(L$ is a complete Riemannian manifold by Claim 14. Take a partition $0=t_{0}<t_{1}<\cdots<t_{k}=1$ such that the length of $\left.\gamma\right|_{\left[t_{l-1}, t_{l}\right]}$ is in $[\varepsilon / 2, \varepsilon)$ for all $l \in\{1, \ldots, k\}$. Observe that $d_{g}(x, y) \geq k \varepsilon / 2$. By 10.3 , for each $l \in\{1, \ldots, k\}$, there is some index $i_{l}$ such that $\left(\gamma\left(t_{l-1}\right), \gamma\left(t_{l}\right)\right) \in R_{i_{l}}$, obtaining

$$
d_{\mathcal{U}}^{*}(x, y)=d_{\mathcal{U}}(x, y) \leq k \leq \frac{2}{\varepsilon} d_{g}(x, y) .
$$

Now, assume that $d_{g}(x, y)<\varepsilon / 2$. Then $(x, y)$ is in some $R_{i}$ by 10.3 , giving $d_{\mathcal{U}}(x, y)=1$, and therefore $d_{\mathcal{U}}^{*}(x, y) \leq 1$. This shows that $d_{\mathcal{U}}^{*} \leq \frac{2}{\varepsilon} d_{g}+1$ on $R_{\mathcal{F}}$, obtaining (ii).

Let $L$ be an arbitrary $\mathcal{F}$-leaf, and $\mathcal{O}$ the corresponding $\mathcal{H}$-orbit. Consider $Z$ as a subset of $X$ via the embedding of $Z$ into $X$ defined by an appropriate choice of the points $b_{i}$ (Section 10.1). In this way, $\mathcal{O}$ becomes a 1 -net in $L$ with $d_{\mathcal{U}}^{*}$. On the other hand, the $\mathcal{U}$-plaques can be identified to the points of $Z$ via the maps $p_{i}$. Moreover, given two different $\mathcal{U}$-plaques, $P$ of $\left(U_{i}, \phi_{i}\right)$ and $Q$ of $\left(U_{j}, \phi_{j}\right)$, we have $P \cap Q \neq \varnothing$ if and only if $p_{i}(P) \in \operatorname{dom} h_{i j}$ and $h_{i j} p_{i}(P)=p_{j}(Q)$. Then, by using plaque chains, it easily follows that $d_{E} \leq d_{\mathcal{U}}^{*} \leq d_{E}+1$ on the subset $\mathcal{O} \subset L$. Thus the

\footnotetext{
${ }^{7}$ This inequality only requires $\mathcal{F}$ to be $C^{1}$.
} 
inclusion map $\left(\mathcal{O}, d_{E}\right) \rightarrow\left(L, d_{\mathcal{U}}^{*}\right)$ is a $(1,1)$-large scale bi-Lipschitz, and its image $\mathcal{O}$ is a 1-net of $\left(L, d_{\mathcal{U}}^{*}\right)$. This gives (iii) by Lemma 2.22 and Proposition 2.27

Remark 10.6. The inclusion maps $\left(\mathcal{O}, d_{E}\right) \rightarrow\left(L, d_{\mathcal{U}}^{*}\right)$ of the proof of Proposition 10.5 (iii) are $(1,3,1)$-large scale Lipschitz equivalences according to Lemma 2.22

By Proposition 10.5 (ii), when $X$ is compact, the $\mathcal{F}$-leaves have a well determined coarse quasi-isometry type of metrics, represented by $d_{\mathcal{U}}^{*}$ for any regular atlas $\mathcal{U}$. Moreover, by Propositions 10.5 (ii) and 2.27, if $X$ is $C^{3}$, the quasi-isometry type of metrics on the leaves can be also represented by $d_{g}$ for any $C^{2}$ Riemannian metric $g$ on $X$.

All coarsely quasi-isometric invariants considered in the Chapter 1 make sense for the leaves with the above coarse quasi-isometry type. Via the identification of $\mathcal{U}$ plaques to points of $Z$ (indicated in the proof of Theorem 10.5 (iii)), the definitions of growth and amenability of the $\mathcal{F}$-leaves given in Chapter 1 , using $\mathcal{U}$-plaques, correspond to the definitions of growth and amenability as metric spaces with $d_{\mathcal{U}}^{*}$, and also to the growth and amenability of the $\mathcal{H}$-orbits with $d_{E}$.

Using the properties indicated in Sections 10.1 and 10.2 and Proposition 10.5 . (iiii), we get Theorems $1.2,1.3$ and $1.6,1.8,1.10$ and 1.12 from Theorems $9.7,9.8 \mid 9.12$ 9.14 9.17 and 9.21, applied to $\mathcal{H}$, because all of those theorems deal with (equi-) coarse quasi-isometric invariants. Similarly, Corollary 1.4 can be obtained from Corollary 9.9 .

The subsets of $X \times X$ and $Z \times Z$ considered in Theorems 1.1 and 9.6 are obviously saturated by $\mathcal{F} \times \mathcal{F}$ and $\mathcal{H} \times \mathcal{H}$, respectively, and moreover they correspond one another by the canonical homeomorphism between the leaf space of $\mathcal{F} \times \mathcal{F}$ and the orbit space of $\mathcal{H} \times \mathcal{H}$. By Corollary 4.10, the subsets of $X \times X$ and $Z \times Z$ considered in Theorems 1.5 and 9.11 are also saturated by $\mathcal{F} \times \mathcal{F}$ and $\mathcal{H} \times \mathcal{H}$, respectively, and correspond one another. Hence, using the properties indicated in Sections 10.1 and 10.2 and Proposition 10.5 (iii), we also get Theorems 1.1 and 1.5 from Theorems 9.6 and 9.11 .

First proof of Theorem 1.13, Let $B$ be a Baire subset of $X$ such that the $\mathcal{F}$-saturation $\mathcal{F}(B)$ is not meager. For each $n \in \mathbb{N}$, let $B_{n}=\cup_{L} \operatorname{Pen}_{\mathcal{U}}(L \cap B, n)$, where $L$ runs in the family of $\mathcal{F}$-leaves. Since $B=B_{0} \subset B_{1} \subset \cdots$ and $\cup_{n} B_{n}=\mathcal{F}(B)$ is not meager, there is some $N \in \mathbb{N}$ such that $B_{N}$ is not meager. Then there is some nonempty open subset $V \subset X$ such that $B_{N} \cap V$ is residual in $V$. By refining $\mathcal{U}$ if necessary, we can assume that this holds with $V=U_{i}$ for some $\left(U_{i}, \phi_{i}\right) \in \mathcal{U}$. By Theorem 8.18 (ii), $C=p_{i}\left(U_{i} \cap B_{N}\right)$ is residual in $Z_{i}$. Hence $\mathcal{H}(C) \cap Z_{i}$ is also residual in $Z_{i}$, and therefore $\mathcal{H}(C)$ is not meager in $Z$. By Theorem 8.28 , it follows that there is some $\mathcal{H}$-saturated residual subset $Y \subset Z$ and some $R>0$ such that $\mathcal{O} \cap C$ is an $R$-net in $\left(\mathcal{O}, d_{E}\right)$ for all orbit $\mathcal{O} \subset Y$. Let $Y^{\prime}$ denote the $\mathcal{F}$-saturated residual subset of $X$ that corresponds to $Y$. Let $\mathcal{O}$ be an $\mathcal{H}$-orbit in $Y$ and $L$ the corresponding $\mathcal{F}$-leaf in $Y^{\prime}$. Consider the embedding of $Z$ into $X$ for an appropriate choice of the points $b_{i}$ (Section 10.1). In this way, according to the proof of Proposition 10.5 (iii), $\mathcal{O} \cap C$ is an $(R+1)$-net in $\left(\mathcal{O}, d_{\mathcal{U}}^{*}\right)$, and $\mathcal{O}$ a 1 -net into $\left(L, d_{\mathcal{U}}^{*}\right)$. Thus $\mathcal{O} \cap C$ becomes an $(R+2)$-net in $L$. Since

$$
\mathcal{O} \cap C \subset \operatorname{Pen}_{\mathcal{U}}\left(L \cap B_{N}, 1\right) \subset \operatorname{Pen}_{\mathcal{U}}(L \cap B, N+1),
$$

we get that $L \cap B$ is an $(R+3+N)$-net in $\left(L, d_{\mathcal{U}}^{*}\right)$. 
Second Proof of Theorem 1.13. Consider first the case where $B$ is open and non-empty. Then the following argument shows that the intersection of all leaves with $B$ are equi-nets in the leaves. If this were wrong, then $B$ would not cut a sequence of balls $B_{\mathcal{U}}\left(x_{n}, r_{n}\right)$ in the leaves with $r_{n} \rightarrow \infty$. Note that $\bigcap_{m} \overline{\cup_{n \geq m} B_{\mathcal{U}}\left(x_{n}, r_{n}\right)}$ is saturated because $r_{n} \rightarrow \infty$. Thus $X=\bigcap_{m} \overline{\bigcup_{n \geq m} B_{\mathcal{U}}\left(x_{n}, r_{n}\right)}$ by the minimality of $\mathcal{F}$, obtaining that the nonempty open set $B$ cuts infinitely many balls $B_{\mathcal{U}}\left(x_{n}, r_{n}\right)$, a contradiction.

In the general case, with the notation of the first proof, there is some $N \in$ $\mathbb{N}$ and a nonempty open subset $V \subset X$ such that $B_{N} \cap V$ is residual in $V$. By increasing $N$ and reducing $V$ if necessary, we can also suppose that $B_{N} \cap V$ is $\left.\mathcal{F}\right|_{V^{-}}$ saturated. Thus $V \backslash B_{N}$ is meager and $\left.\mathcal{F}\right|_{V}$-saturated. So $\mathcal{F}\left(V \backslash B_{N}\right)$ is meager by Lemma 10.3 (ii), and therefore the saturated set $Y=X \backslash \mathcal{F}\left(V \backslash B_{N}\right)$ is residual. Since $L \cap B_{N} \cap V=L \cap V$ for any leaf $L$ in $Y$, it follows that the intersections of all leaves in $Y$ with $B_{N}$ are equi-nets in those leaves. Then the same property is satisfied with $B$ like in the first proof.

\subsection{Higson corona of the leaves}

10.4.1. Higson compactification. Let $L$ be a leaf of $\mathcal{F}$. To simplify the notation, let $L_{\mathcal{U}}=\left(L, d_{\mathcal{U}}^{*}\right)$, whose Higson compactification is denoted by $L_{\mathcal{U}}^{\nu}$. If $\mathcal{V}$ is another regular foliated atlas of $\mathcal{F}$, then the identity map $L_{\mathcal{U}} \rightarrow L_{\mathcal{V}}$ is a large scale bi-Lipschitz bijection (Proposition 10.5-(i)). Therefore it induces a map $L_{\mathcal{U}}^{\nu} \rightarrow L_{\mathcal{V}}^{\nu}$, which is continuous at the points of $\nu L$ and restricts to a homeomorphism between the corresponding coronas (Proposition 7.4 (ii),(iii)). Thus the corona of $L_{\mathcal{U}}^{\nu}$ will be simply denoted by $\nu L$. The notation $L^{\nu}$ will be used for the underlying set of $L_{\mathcal{U}}^{\nu}$ equipped with the coarsest topology so that the identity map $L^{\nu} \rightarrow L_{\mathcal{U}}^{\nu}$ is continuous and the inclusion map $L \rightarrow L^{\nu}$ is an open embedding. The space $L^{\nu}$ is a compactification of $L$, called its Higson compactification, whose corona is $\nu L$. If $\mathcal{F}$ is $C^{3}$ and $g$ is a $C^{2}$ Riemannian metric on $X$, then the Higson compactification of $\left(L, d_{g}\right)$ is $L^{\nu}$.

Let $\mathcal{O}$ be the $\mathcal{H}$-orbit that corresponds to $L$, equipped with $d_{E}$. Then $\mathcal{O}$ becomes a subspace of both $L_{\mathcal{U}}$ and $L$ with the injection $Z \rightarrow X$ given in Section 10.1 . According to the proof of Proposition 10.5 (iii), the inclusion map $\mathcal{O} \rightarrow L_{\mathcal{U}}$ is $(1,1)$ large scale bi-Lipschitz and its image is a 1-net. Thus $\mathcal{O} \hookrightarrow L$ induces an embedding $\mathcal{O}^{\nu} \rightarrow L_{\mathcal{U}}^{\nu}$ (Corollary 7.6), which restricts to a homeomorphism $\nu \mathcal{O} \rightarrow \nu L$ (Remark 10.6 and Proposition 7.4.(iii)). If the above map $\mathcal{O}^{\nu} \rightarrow L_{\mathcal{U}}^{\nu}$ is considered as map $\mathcal{O} \rightarrow L^{\nu}$, then it is also an embedding because $\mathcal{O}$ is subspace $L$. In this way, $\mathcal{O}^{\nu}$ becomes a subspace of both $L_{\mathcal{U}}^{\nu}$ and $L^{\nu}$, with $\nu \mathcal{O}=\nu L$. Hence Theorem 1.11 is a direct consequence of Theorem 9.32 .

Using Lemma 7.2, it easily follows that, for any compactification $\bar{L} \leq L^{\nu}$, there are unique compactifications, $\bar{L}_{\mathcal{U}} \leq L_{\mathcal{U}}^{\nu}$ and $\overline{\mathcal{O}} \leq \mathcal{O}_{\mathcal{U}}^{\nu}$, such that the identity map $L \rightarrow L_{\mathcal{U}}$ and inclusion map $\mathcal{O} \rightarrow L_{\mathcal{U}}^{\nu}$ have continuous extensions $\bar{L} \rightarrow \bar{L}_{\mathcal{U}}$ and $\overline{\mathcal{O}} \rightarrow \bar{L}_{\mathcal{U}}$ that restrict to the identity map on $\partial L=\partial \mathcal{O}$. In fact, $\overline{\mathcal{O}}=\mathrm{Cl}_{\bar{L}_{\mathcal{U}}}(\mathcal{O})=\mathrm{Cl}_{\bar{L}}(\mathcal{O})$.

Consider the pseudogroup $\widetilde{\mathcal{H}}$ on $\widetilde{Z}=\bigsqcup_{i} \widetilde{Z}_{i}$, with symmetric set of generators $\widetilde{E}=\left\{\tilde{h}_{i j}\right\}$, induced by the foliated atlas $\widetilde{\mathcal{U}}$ (Section 10.1). By refining $\widetilde{\mathcal{U}}$ if necessary, we can assume that this foliated atlas is also regular. Then $\widetilde{E}$ is also a recurrent system of compact generation on $\widetilde{Z}$ of another compactly generated pseudogrup on 
a larger space (Lemma 10.4). The points $b_{i}$ also define a map $\widetilde{Z} \rightarrow X$, which can be assumed to be the embedding. Thus we get the subspace inclusions $Z \subset \widetilde{Z} \subset X$.

Let $\widetilde{\mathcal{O}}$ be the $\widetilde{\mathcal{H}}$-orbit that corresponds to $L$, equipped with $d_{\widetilde{E}}$. As before, there are unique compactification $\overline{\widetilde{\mathcal{O}}} \leq \widetilde{\mathcal{O}}^{\nu}$, with corona $\partial \widetilde{\mathcal{O}}$, where $\overline{\widetilde{\mathcal{O}}}=\mathrm{Cl}_{\bar{L}}(\widetilde{\mathcal{O}})=\mathrm{Cl}_{\bar{L}_{\mathcal{U}}}(\widetilde{\mathcal{O}})$ and $\partial \widetilde{\mathcal{O}}=\partial L$. Furthermore, by Proposition 8.9, the inclusion map $\mathcal{O} \rightarrow \widetilde{\mathcal{O}}$ is biLipschitz and its image is a net. Thus, as above, it induces an embedding $\overline{\mathcal{O}} \rightarrow \overline{\widetilde{\mathcal{O}}}$, which restricts to a homeomorphism $\partial \mathcal{O} \rightarrow \partial \widetilde{\mathcal{O}}$. In this way, we will consider $\overline{\mathcal{O}}$ as a subspace of $\overline{\widetilde{\mathcal{O}}}$, with $\partial \mathcal{O}=\partial \widetilde{\mathcal{O}}$; indeed, as explained before, $\overline{\mathcal{O}}=\mathrm{Cl}_{\bar{L}}(\mathcal{O}) \subset \mathrm{Cl}_{\bar{L}}(\widetilde{\mathcal{O}})=$ $\overline{\widetilde{\mathcal{O}}}$ with $\partial \mathcal{O}=\partial L=\partial \widetilde{\mathcal{O}}$.

10.4.2. Limit sets. Let us continue with the notation of Section 10.4.1

Definition 10.7. The limit set of $L$ at any $\mathbf{e} \in \partial L$, denoted by ${ }^{8} \lim _{\mathbf{e}} L$, is the subset $\cap_{V} \mathrm{Cl}_{X}(V \cap L)$ of $X$, where $V$ runs in the collection of neighborhoods of $\mathbf{e}$ in $\bar{L}$.

Like in the case of pseudogroups (Section 9.5.1), higher compactifications of the leaves induce smaller limit sets. Moreover lime is closed and nonempty ${ }^{9}$, which may not be $\mathcal{F}$-saturated. The following examples are foliated versions of Examples 9.23 ,

EXAMPLES 10.8. (i) For the one-point compactification $L^{*}$, the limit set of $L$ at the unique point in the corona is the standard limit set of $L$, which is saturated.

(ii) For the compactification of $L$ by the end space, we get the standard limit set of $L$ at any end of $L$, which is also a saturated set.

(iii) Consider the set

$$
\bar{L}=L \sqcup \mathrm{Cl}_{X}(L)=(L \times\{0\}) \cup\left(\mathrm{Cl}_{X}(L) \times\{1\}\right)
$$

with the topology determined as follows: $L \equiv L \times\{0\} \hookrightarrow \bar{L}$ is an open embedding of the leaf, and, a basic neighborhood of a point in $(x, 1) \in \mathrm{Cl}_{X}(L) \times\{1\}$ in $\bar{L}$ is of the form $(V \cap L) \sqcup V$, where $V$ is any neighborhood of $x$ in $\mathrm{Cl}_{X}(U)$. This $\bar{L}$ is a compactification of $L \equiv L \times\{0\}$. In terms of algebras of functions, $\bar{L}$ corresponds to the algebra of $\mathbb{C}$-valued functions on $L$ that admit a continuous extension to $\mathrm{Cl}_{X}(L)$. The corona of $\bar{L}$ is $\partial L=\mathrm{Cl}_{X}(L) \times\{1\} \equiv \mathrm{Cl}_{X}(L)$. Moreover, for each $x \in \partial L$, it is easy to see that $\lim _{x} L=\{x\}$, which is not saturated if $\operatorname{dim} \mathcal{F}>0$. (An analytic application of this compactification is given in [22.)

(iv) For the Stone-Čech compactification $L^{\beta}$, the limit set of $L$ at any point in the corona $L^{\beta}$ is a singleton by (iii).

(v) If $\bar{L} \leq L^{\nu}$, it will be shown that $\lim _{\mathbf{e}} L$ is $\mathcal{F}$-saturated for all $\mathbf{e} \in \partial L$ (Theorems 9.25 and 10.10 .

(vi) As a particular case of $(\mathrm{V})$, suppose that $\mathcal{F}$ is $C^{\infty}$ and $g$ is a $C^{\infty}$ Riemannian metric on $X$ so that $(L, g)$ has negative curvature. Then we can consider the

\footnotetext{
${ }^{8}$ When $\partial L$ is the end space of $L$ (Example 10.8-(ii)), it is standard to use the term e-limit of $L$ and the notation $\mathbf{e}-\lim L$. We prefer the stated terminology and notation because this concept represents the limit of the inclusion map $L \hookrightarrow X$ at $\mathbf{e}$ (a formalization of a point at the "infinity"

${ }^{9}$ If the compactness assumption on $X$ is removed, then $\lim$ e can be defined as well, but it may be empty.
} of $L$ ). 
compactification of $L$ whose corona is the ideal boundary. The limit sets of $L$ at points in its ideal boundary are $\mathcal{F}$-saturated.

For $S \subset X$ and $r \geq 0$, the penumbrd ${ }^{10}$ of $S$ of radius $r$ is the set

$$
\operatorname{Pen}_{\mathcal{U}}(S, r)=\bigcup_{x \in S} \bar{B}_{\mathcal{U}}(x, r) .
$$

Observe that $\operatorname{Pen}_{\mathcal{U}}(S, 1)$ is the union of the $\mathcal{U}$-plaques that meet $S$.

Lemma 10.9. For all $\mathbf{e} \in \partial L$,

$$
\operatorname{Pen}_{\mathcal{U}}\left(\lim _{\mathbf{e}} \mathcal{O}, 1\right) \subset \lim _{\mathbf{e}} L \subset \operatorname{Pen}_{\widetilde{\mathcal{U}}}\left(\operatorname{Pen}_{\widetilde{E}}\left(\lim _{\mathbf{e}} \widetilde{\mathcal{O}}, 1\right), 1\right)
$$

Proof. Since the inclusion map $\mathcal{O} \rightarrow L_{\mathcal{U}}$ is $(1,1)$-large scale bi-Lipschitz and its image is a 1-net, it is a $(1,3,1)$-large scale Lipschitz equivalence (Remark 10.6). Thus, by Proposition 7.11, given a base $\mathcal{V}$ of neighborhoods of $\bar{e}$ in $\mathcal{O}^{\nu}$, the sets $V^{\prime}:=\mathrm{Cl}_{L_{\mathcal{U}}^{\nu}}\left(\operatorname{Pen}_{\mathcal{U}}(V \cap \mathcal{O}, 1)\right)$, with $V \in \mathcal{V}$, form a base $\mathcal{V}^{\prime}$ of neighborhoods of e in $L^{\nu}$. Since $\mathcal{O}$ is a net in $L_{\mathcal{U}}$ and each $\mathcal{U}$-plaque is closed in $L_{\mathcal{U}}$, it easily follows that $\operatorname{Pen}_{L_{\mathcal{U}}}(V \cap \mathcal{O}, 1)$ is closed in $L_{\mathcal{U}}$, and therefore

$$
V^{\prime} \cap L=\operatorname{Pen}_{\mathcal{U}}(V \cap \mathcal{O}, 1) .
$$

Claim 15. For all $V \in \mathcal{V}$,

$$
\mathrm{Cl}_{X}\left(V^{\prime} \cap L\right) \subset \operatorname{Pen}_{\widetilde{\mathcal{U}}}\left(\mathrm{Cl}_{\widetilde{Z}}\left(\operatorname{Pen}_{E}(V \cap \mathcal{O}, 1)\right), 1\right) .
$$

So any $x \in \mathrm{Cl}_{X}\left(V^{\prime} \cap L\right)$ is the limit in $X$ of some sequence $x_{k} \in V^{\prime} \cap L$. By 10.4), there are sequences of points $y_{k} \in V \cap \mathcal{O}$, and indices $i_{k}$ and $j_{k}$, such that $x_{k} \in U_{i_{k}}$, $y_{k} \in U_{i_{k}} \cap \mathcal{O} \cap Z_{j_{k}}$, and $p_{i_{k}}\left(x_{k}\right)=p_{i_{k}}\left(y_{k}\right):=z_{k} \in Z_{i_{k}}$ for all $k$. Then $z_{k}=h_{j_{k} i_{k}}\left(y_{k}\right) \epsilon$ $\operatorname{Pen}_{E}(V \cap \mathcal{O}, 1)$. Since $\mathcal{U}$ is finite, each $U_{i}$ is relatively compact in $\widetilde{U}_{i}$, and each $Z_{i}$ is a relatively compact in $\widetilde{Z}_{i}$, by passing to a subsequence if necessary, we can assume that there is an index $i$, and points $y \in \widetilde{U}_{i}$ and $z \in \widetilde{Z}_{i}$ such that $i_{k}=i$ for all $k$, and $y_{k} \rightarrow y$ in $\widetilde{U}_{i}$ and $z_{k} \rightarrow z$ in $\widetilde{Z}_{i}$ as $k \rightarrow \infty$. Thus $y \in \mathrm{Cl}_{\widetilde{Z}}\left(\operatorname{Pen}_{E}(V \cap \mathcal{O}, 1)\right)$. Moreover $x \in \widetilde{U}_{i}$ and $\tilde{p}_{i}(x)=\lim _{k} \tilde{p}_{i}\left(x_{k}\right)=\lim _{k} z_{k}=z$, obtaining that $d_{\widetilde{u}}^{*}(x, y) \leq 1$. This shows Claim 15

Claim 16. For all $V \in \mathcal{V}$,

$$
\operatorname{Pen}_{\mathcal{U}}\left(\mathrm{Cl}_{Z}(V \cap \mathcal{O}), 1\right) \subset \mathrm{Cl}_{X}\left(V^{\prime} \cap L\right) .
$$

For $x \in \operatorname{Pen}_{\mathcal{U}}\left(\mathrm{Cl}_{Z}(V \cap \mathcal{O}), 1\right)$, there is some $y \in \mathrm{Cl}_{Z}(V \cap \mathcal{O})$ and some index $i$ such that $x, y \in U_{i}$ and $p_{i}(x)=p_{i}(y)=: z \in Z_{i}$. Moreover, for some $j$ so that $y \in Z_{j}$, there is some sequence $y_{k} \in V \cap \mathcal{O} \cap U_{i} \cap Z_{j}$ such that $y_{k} \rightarrow y$ in $Z_{j}$ as $k \rightarrow \infty$. Since $p_{i}(x)=z=p_{i}(y)=\lim _{k} p_{i}\left(y_{k}\right)$, there is some sequence $x_{k} \in U_{i}$ such that $p_{i}\left(x_{k}\right)=p_{i}\left(y_{k}\right)$ and $x_{k} \rightarrow x$ as $k \rightarrow \infty$. Thus $d_{\mathcal{U}}\left(x_{k}, y_{k}\right)=1$, giving $x_{k} \in V^{\prime} \cap L$ by (10.4), and therefore $x \in \mathrm{Cl}_{X}\left(V^{\prime} \cap L\right)$. This proves Claim 16 .

Now the result follows from Claims 15 and 16 by taking intersections with $V$ running in $\mathcal{V}$.

The following is a more explicit version of Theorem 1.16 .

\footnotetext{
${ }^{10}$ We can consider $d_{\mathcal{U}}$ as a "metric with possible infinite" values on $X$, defining $d_{\mathcal{U}}(x, y)=\infty$ when $L_{x} \neq L_{y}$. Then this definition of penumbra is the direct extension of the above one to "metrics with possible infinite values."
} 
Theorem 10.10. Let $(X, \mathcal{F})$ be a compact Polish foliated space, let $\bar{L}$ be a compactification of an $\mathcal{F}$-leaf $L$, with corona $\partial L$, let $\mathcal{H}$ be the representative of the holonomy pseudogroup induced by a regular foliated atlas, and let $\mathcal{O}$ be the $\mathcal{H}$-orbit that corresponds to L. Suppose that $\bar{L} \leq L^{\nu}$, and let $\overline{\mathcal{O}} \leq \mathcal{O}^{\nu}$ be the compactification of $\mathcal{O}$ that corresponds to $\bar{L}$, with corona $\partial \mathcal{O}=\partial L$. Then $\lim _{\mathbf{e}} L=\mathcal{F}\left(\lim _{\mathbf{e}} \mathcal{O}\right)$ for all $\mathbf{e} \in \partial L$.

Proof. From Lemma 10.9, Theorem 9.25 and Proposition 9.30, we get

$$
\mathcal{F}\left(\lim _{\mathbf{e}} \mathcal{O}\right) \subset \lim _{\mathbf{e}} L \subset \mathcal{F}\left(\lim _{\mathbf{e}} \widetilde{\mathcal{O}}\right)=\mathcal{F}\left(\widetilde{\mathcal{H}}\left(\lim _{\mathbf{e}} \mathcal{O}\right)\right)=\mathcal{F}\left(\lim _{\mathbf{e}} \mathcal{O}\right) .
$$

Now, Theorems 1.15 and 1.16 follow from Theorems $9.28,9.29$ and 10.10 .

\subsection{Algebraic asymptotic invariants}

Let $\mathrm{Top}_{\sigma}$ denote the category of $\sigma$-compact topological spaces and surjective continuous maps, let $\mathbb{A}$ be a category with limits, and let $F: \operatorname{Top}_{\sigma} \rightarrow \mathbb{A}$ be a functor which is continuous in the following sense: For each object $X$ in $\operatorname{Top}_{\sigma}$ and each increasing sequence $K_{n}$ of compact subspaces of $X, F\left(\cup_{n} K_{n}\right)=\lim _{n} F\left(K_{n}\right)$, where the limit is injective or projective according to whether $F$ is covariant or contravariant.

If $F$ is a continuous functor, there is an associated functor $F^{\infty}$, the limit of $F$ at infinity, which is defined as follows. If $X$ is an object in $\operatorname{Top}_{\sigma}$, then

$$
F^{\infty}(X)=\lim _{K} F(X \backslash K)
$$

and if $f: X \rightarrow Y$ is surjective, then

$$
F^{\infty}(f)=\lim _{K} F(X \backslash K \rightarrow Y \backslash K)
$$

where $K$ denotes the family of compact subsets of $X$.

THEOREM 10.11. Let $F$ be a continuous functor on $\mathrm{Top}_{\sigma}$ with values in the category of vector spaces over a field. Let $X$ be a transitive foliated space with no holonomy. Then the map $\operatorname{dim}: X \rightarrow \mathbb{N} \cup\{\infty\}$ which assigns to $x \in X$ the dimension of $F^{\infty}\left(L_{x}\right)$ is Borel, and is constant on a residual set of leaves.

Proof. Assume that the functor $F$ is covariant. If $x \in X$, let $B(x, r)$ denote the ball of radius $r$ in the leaf containing $x$. For positive integers $n, m$, and $i<j<k<l$, let $Y_{n}(i, j, k, l, m)$ denote the set of points $x \in X$ for which there are compact domains

$$
\bar{B}(x, k) \subset \Omega_{x, k, m} \subset \bar{B}(x, k+1 / m), \quad \bar{B}(x, l) \subset \Omega_{x, l, m} \subset \bar{B}(x, l+1 / m),
$$

and open domains

$$
\bar{B}(x, j-1 / m) \subset U_{x, j, m} \subset \bar{B}(x, j), \quad \bar{B}(x, i-1 / m) \subset U_{x, i, m} \subset \bar{B}(x, i),
$$

such that the map

$$
F\left(\Omega_{x, k, m} \backslash U_{x, j, m} \rightarrow \Omega_{x, l, m} \backslash U_{x, i, m}\right)
$$

has image of dimension $\geq n$. The following assertion follows from the local Reeb stability for foliated spaces [23, Proposition 11.4.8].

Claim 17. The sets $Y_{n}(i, j, k, l, m)$ are closed in $X$. 
For each leaf $L$ of $X$ and each point $x \in L$, we have that

$$
F^{\infty}(L)=\lim _{\leftarrow} \lim _{k>i} F(\bar{B}(x, k) \backslash B(x, i)),
$$

and so the set of points $x \in X$ where $\operatorname{dim} F^{\infty}\left(L_{x}\right) \geq n$ is

$$
Y_{n}=\bigcup_{i} \bigcap_{j>i} \bigcup_{k>j} \bigcap_{l>k} \bigcap_{m} Y_{n}(i, j, k, l, m) .
$$

Hence the function $\operatorname{dim}: X \rightarrow \mathbb{N} \cup\{\infty\}$, which is constant along the leaves, has the property that $\operatorname{dim}^{-1}[n, \infty]$ is Borel for each $n \in \mathbb{N}$ by Claim 17 and so $\operatorname{dim}^{-1}\{n\}$ is a Borel saturated subset of $X$ for each $n \in \mathbb{N}$. The transitivity hypothesis on $X$ in turn implies that the function dim is constant on a residual saturated subset of $X$.

\subsection{Versions with quasi-invariant currents}

Let $(X, \mathcal{F})$ be a foliated space of class $C^{2}$. Then the space of densities on $(X, \mathcal{F})$ is a foliated space of class $C^{1}$, and a bundle over $(X, \mathcal{F})$. A current, $m$, on $(X, \mathcal{F})$ is a positive linear functional on the space of compactly supported densities on $X$. It is called a quasi-invariant current if on each foliation chart $\phi: U \rightarrow B \times Z$, the current $\phi_{*} m$ on $B \times Z$ admits a disintegration of the form

$$
\phi_{*} m=\int_{Z} \lambda_{z} \cdot \mu_{Z}(z),
$$

where $\mu_{Z}$ is a measure on the transversal $Z$ and, for $\mu_{Z}$-almost all $z \in Z, \lambda_{z}$ is equivalent to the Lebesgue current on the plaque $B \times\{z\}$. The measures $\left\{\mu_{Z}\right\}$ on the transversals are quasi-invariant under the holonomy pseudogroup of $(X, \mathcal{F})$, so they define quasi-invariant measure class for the holonomy pseudogroup of $(X, \mathcal{F})$.

EXAMPLES 10.12. (i) A transverse invariant measure combined with the current of integration along the leaves defines a quasi-invariant current on the foliated space.

(ii) If the space $X$ is a $C^{1}$ manifold so that $(X, \mathcal{F})$ is the foliated space having leaves the connected components of $X$, then the Lebesgue current of integration is a quasi-invariant measure on $(X, \mathcal{F})$.

These two examples are certainly not common among foliated spaces. A foliated space is rarely a manifold, and the generic foliated space lacks an invariant measure. Nevertheless, quasi-invariant currents do exist on any compact foliated space.

Suppose that $(X, \mathcal{F})$ is differentiable of class $C^{2}$, let $g$ be a $C^{1}$ leafwise Riemannian metric on $X$, and let $\Delta$ denote the Laplacian defined by $g$ on the leaves, mapping $C^{2}$ functions on $X$ to continuous functions. A Borel measure $m$ on $X$ is called harmonic if $m(\Delta f)=0$ for all $C^{2}$ function $f$ on $X$. The Riemannian metric determines a volume density on $(X, \mathcal{F})$ and induces an identification of densities on $(X, \mathcal{F})$ with functions on $X$. Therefore a harmonic measure $m$ gives rise to a quasi-invariant current which has the following local structure: on any foliated chart $U \cong B \times Z, m$ admits a disintegration of the form

$$
m(f)=\int_{Z} \int_{B \times\{z\}} h(x, z) f(x, z) \mu(z)
$$

where $h(\cdot, z)$ is a positive harmonic fuction on $B \times\{z\}$, for $\mu$-almost all $z \in Z$.

Harmonic measures were introduced by Garnett [47, who proved that they satisfy several relevant properties (see also [22, [24, Chapter 2]). For instance, if 
$X$ is compact, there exists some harmonic probability measure on $X$. A harmonic measure on $X$ is called ergodic (or $\mathcal{F}$-ergodic) if every $\mathcal{F}$-saturated set is either of zero measure or of full measure. Any $\mathcal{H}$-invariant measure on $Z$ (a transverse invariant measure of $\mathcal{F}$ ) induces a harmonic measure on $X$. On the other hand, any harmonic measure $m$ on $X$ induces an $\mathcal{H}$-invariant measure class $[\nu]$ on $Z$ so that the $\mathcal{F}$-saturated sets of $m$-zero measure correspond to $\mathcal{H}$-saturated sets of [ $\nu]$-zero measure, and $[\nu]$ is $\mathcal{H}$-ergodic if and only $\mu$ is $\mathcal{F}$-ergodic.

Hamonic measures have good recurrence properties. A leaf in a foliated space is called a wandering leaf if it is proper and not compact. The wandering set of a foliated space is the union of all its wandering leaves, and the nonwandering set is its complement. Both sets are saturated Borel sets.

A theorem of Garnett [47 states that the wandering set of a foliated space has full measure with respect to any harmonic measure.

For a foliated space, $X$, the subset of leaves without holonomy, $X_{0} \subset X$, may consist entirely of wandering leaves and thus be of measure zero for any harmonic measure on $X$. This is for example the case of proper foliations (a well studied class of foliations of codimension one), where the nonwandering set consists of only compact leaves. There are also large classes of minimal, foliated spaces where the set $X_{0}$ has full measure. One such class is when $X$ arises as a Markov exceptional minimal set of a codimension one foliation [30; for these, there are at most countably many leaves with holonomy, so $X \backslash X_{0}$ has measure zero (with respect to any quasi-invariant transverse measure class) because its intersection with any foliation chart is a countable set of plaques.

The following results follow directly from Theorems 9.349 .38 .

Theorem 10.13. Let $(X, \mathcal{F})$ be a compact Polish foliated space. With respect to an ergodic quasi-invariant current on $X$ for which $X \backslash X_{0}$ has zero measure, either

(i) almost all $\mathcal{F}$-leaves are coarsely quasi-isometric to almost all $\mathcal{F}$-leaves; or else (ii) almost all $\mathcal{F}$-leaves are coarsely quasi-isometric to almost no $\mathcal{F}$-leaf.

Theorem 10.14. Let $(X, \mathcal{F})$ be a compact Polish foliated space. With respect to an ergodic quasi-invariant current on $X$ for which $X \backslash X_{0}$ has zero measure, either

(i) almost all $\mathcal{F}$-leaves have the same growth type; or else

(ii) the growth type of almost all $\mathcal{F}$-leaves are comparable with the growth type of almost no $\mathcal{F}$-leaf.

Theorem 10.15. Let $(X, \mathcal{F})$ be a compact Polish foliated space. With respect to an ergodic quasi-invariant current on $X$ for which $X \backslash X_{0}$ has zero measure, the equalities and inequalities of Theorem 1.8 are satisfied almost everywhere with some $a_{1}, a_{3} \in[1, \infty], a_{2}, a_{4} \in[0, \infty)$ and $p \geq 1$.

Corollary 10.16. Let $(X, \mathcal{F})$ be a compact Polish foliated space. With respect to an ergodic quasi-invariant current on $X$ for which $X \backslash X_{0}$ has zero measure, any of the sets of Corollary 1.9 is either of zero measure or of full measure.

Theorem 10.17. Let $(X, \mathcal{F})$ be a compact Polish foliated space. With respect to an ergodic harmonic measure on $X$, for which $X \backslash X_{0}$ has zero measure, almost all leaves have the same asymptotic dimension. 


\subsection{There is no measure theoretic version of recurrence}

We show that there is no measure theoretic version of Theorem 1.13. It fails for the most simple non-trivial minimal foliation: a minimal Kronecker flow on the 2-torus. Let us first prove the measure theoretic version of its pseudogroup counterpart (Theorem 8.28 fails for the pseudogroup generated by a rotation with dense orbits on the unit circle.

Let $h$ be a rotation of the unit circle $S^{1} \subset \mathbb{C} \equiv \mathbb{R}^{2}$ with dense orbits. Consider the action of $\mathbb{Z}$ on $S^{1}$ induced by $h$ (the action of each $n \in \mathbb{Z}$ is given by $h^{n}$ ). Consider the standard Riemannian metric on $S^{1}$, and let $\Lambda$ be the corresponding Riemannian measure. Thus the action is isometric and $\Lambda$ is invariant. Moreover $\Lambda$ is ergodic [36].

Let $\mathcal{H}$ be the minimal pseudogroup on $S^{1}$ generated by the above action, which satisfies the conditions of Theorem 8.28, taking $U=S^{1}, \mathcal{G}=\mathcal{H}$ and $E=\left\{h, h^{-1}\right\}$. For each positive integer $n$, let $I_{n}$ be an open arc in $S^{1}$ with $\Lambda\left(I_{n}\right)<\frac{1}{(2 n+1) 2^{n}}$. Then

$$
A=\bigcup_{n=1}^{\infty} \bigcup_{i=-n}^{n} h^{i}\left(I_{n}\right)
$$

is a Borel set with

$$
\Lambda(A) \leq \sum_{n=1}^{\infty} \sum_{i=-n}^{n} \Lambda\left(h^{i}\left(I_{n}\right)\right)=\sum_{n=1}^{\infty} \frac{1}{2^{n}}=1<2 \pi=\Lambda\left(S^{1}\right) .
$$

So its complement $B=S^{1} \backslash A$ is a Borel set with $\Lambda(B)>0$, and thus $\Lambda(\mathcal{H}(B))=$ $\Lambda\left(S^{1}\right)$ because $\Lambda$ is ergodic. Nevertheless, every orbit $\mathcal{O}$ meets each $I_{n}$ at some point $x$, and thus

$$
\mathcal{O} \cap A \supset\left\{h^{i}(x) \mid-n \leq i \leq n\right\}=\bar{B}_{E}(x, n) .
$$

Hence $\mathcal{O} \cap B$ is not a net in $\left(\mathcal{O}, d_{E}\right)$ for any orbit $\mathcal{O}$.

The suspension (Section 11.1) of the above action produces a minimal Kronecker flow on the 2-torus. Equip $\mathbb{R}$ with the standard Riemannian metric. Then the universal cover $\mathbb{R} \rightarrow S^{1}, t \mapsto e^{2 \pi t i}$, is a local isometry. Let $\widetilde{X}=\mathbb{R} \times S^{1}, \tilde{g}$ the product Riemannian metric on $\widetilde{X}$, and $\widetilde{\mathcal{F}}$ the $C^{\infty}$ foliation on $\widetilde{X}$ whose leaves are the fibers $\mathbb{R} \times\{x\}$ for $x \in S^{1}$. The Riemannian measure $\tilde{\mu}$ of $\tilde{g}$ is harmonic for $\widetilde{\mathcal{F}}$ with respect to the restriction of $\tilde{g}$ to the leaves. Consider the $C^{\infty}$ diagonal $\mathbb{Z}$-action on $\widetilde{X}$, given by $n \cdot(t, x)=\left(n+r, h^{n}(x)\right)$, which is isometric and preserves $\widetilde{\mathcal{F}}$. Moreover $X=\mathbb{Z} \backslash \widetilde{X}$ is a $C^{\infty}$ manifold so that the quotient map $p: \widetilde{X} \rightarrow X$ is a $C^{\infty}$ covering map. Thus $\tilde{g}$ and $\widetilde{\mathcal{F}}$ project to a Riemannian metric $g$ and a $C^{\infty}$ foliation $\mathcal{F}$ on $X$, and the Riemannian measure $\mu$ of $g$ is harmonic for $\mathcal{F}$ with respect to the restriction of $g$ to the leaves. Note that $\mathcal{H}$ is a representative of the holonomy pseudogroup of $\mathcal{F}$. Observe that the restriction $p:[0,1 / 2] \times B \rightarrow p([0,1 / 2] \times B)=: B^{\prime}$ is bijective. So

$$
\mu\left(B^{\prime}\right)=\tilde{\mu}([0,1 / 2] \times B)=\frac{\Lambda(B)}{2}>0 .
$$

On the other hand, suppose that there is an $\mathcal{F}$-leaf $L$ so that $L \cap B^{\prime}$ is a $K$-net in $L$ for some $K \in \mathbb{N}$. Since $\mathcal{H}$ is minimal, there is some $x \in I_{K+1}$ such that $L=p(\mathbb{R} \times\{x\})$. Then $L \cap B^{\prime}=p\left((\mathbb{R} \times\{x\}) \cap p^{-1}\left(B^{\prime}\right)\right)$. Since the restriction $p: \widetilde{L} \rightarrow L$ is an isometry with the restrictions of $\tilde{g}$ and $g$, it follows that

$$
(\mathbb{R} \times\{x\}) \cap p^{-1}\left(B^{\prime}\right)=\bigcup_{h^{-i}(x) \in B}[i, i+1 / 2]
$$


is a $K$-net in $\mathbb{R}$. But, by $10.5, h^{i}(x) \notin B$ for $-K-1 \leq i \leq K+1$ because $x \in I_{K+1}$, and therefore 0 is not in the $K$-penumbra of $\bigcup_{h^{-i}(x) \in B}[i, i+1 / 2]$ in $\mathbb{R}$, a contradiction. Thus $L \cap B^{\prime}$ is not a net in $L$ for all $\mathcal{F}$-leaf $L$. 



\section{CHAPTER 11}

\section{Examples and open problems}

This chapter is mainly devoted to illustrate our main results with examples. To begin with, we recall recall some basic constructions of foliated spaces. Then we recall a procedure that allows to realize any connected Riemannian manifold of bounded geometry as a leaf of a compact Riemannian foliated space without holonomy. Besides of its theoretical interest, it can be used as a practical way of produce examples. This realization relies on a version using graphs instead of Riemannian manifolds. Indeed graphs can be also use to produce foliated spaces in a more direct way, specially using Cayley graphs. Then we recall and provide concrete examples, where our main theorems are confirmed. For this purpose, we have to recall some more concepts from descriptive set theory and theory of levels.

A few open problems are also included in the last section.

\subsection{Foliated spaces defined by suspensions}

Let $\pi: \widetilde{L} \rightarrow L$ be a regular $\Gamma$-covering of a closed Riemannian manifold, and consider an action of $\Gamma$ on a Polish space $Z$. Such a group $\Gamma$ is finitely generated. We get a diagonal action of $\Gamma$ on $\widetilde{L} \times Z$, defined by $\gamma \cdot(\tilde{y}, z)=(\gamma \cdot \tilde{y}, \gamma \cdot z)$. This diagonal action preserves the trivial foliation with leaves $\widetilde{L} \times\{z\}(z \in Z)$, and therefore it induces a foliated structure on the Polish space $X=\Gamma \backslash(\widetilde{L} \times Z)$, which is called a suspension foliated space. Moreover the first factor projection $\widetilde{L} \times Z \rightarrow \widetilde{L}$ induces a fiber bundle projection $X \rightarrow L$ with typical fiber $Z$, whose fibers are transverse to the leaves. The holonomy pseudogroup of $X$ can be represented by the pseudogroup generated by the action of $\Gamma$ on $Z$. Thus $X$ is transitive or minimal if so is the action of $\Gamma$ on $Z$. For every orbit $\Gamma \cdot z(z \in Z)$, the corresponding leaf $L$ of $X$ is the projection of $\widetilde{L} \times\{z\}$. Recall that $\Gamma \cdot z \equiv \Gamma / \Gamma_{z}$, where $\Gamma_{z}$ is the isotropy subgroup.

If $Z$ is compact, then $X$ is also compact, and $L$ is coarsely quasi-isometric to $\Gamma \cdot z \equiv \Gamma / \Gamma_{z}$. So the leaves are quasi-isometric to $\Gamma$ if the isotropy groups are trivial. More generally, two leaves are coarsely quasi-isometric if the corresponding conjugacy classes of isotropy groups have commensurable representatives. Thus, in this setting, the relation of commensurability up to conjugation is an interesting refinement of the coarse quasi-isometry relation between leaves.

Any action of a finitely generated group $\Gamma$ on a compact space $Z$ can be used to produce a compact suspension foliated space. If $\Gamma$ can be generated by $n$ elements $\left(n \in \mathbb{Z}^{+}\right)$, then it is a quotient of the free group $F_{n}$ with $n$ generators. Thus any action of $\Gamma$ on a compact space $Z$ can be transformed into an $F_{n}$ action. On the other hand, the closed orientable surface $\Sigma_{n}$ of genus $n$ has an obvious $F_{n}$ regular covering $\widetilde{\Sigma}_{n}$. This gives rise to the suspension foliated space $X=F_{n} \backslash\left(\widetilde{\Sigma}_{n} \times Z\right)$. If $K \triangleleft F_{2}$ is the kernel of the projection $F_{n} \rightarrow \Gamma$, then $\widetilde{\Sigma}_{n}^{\prime}=K \backslash \widetilde{\Sigma}_{n}$ is a regular $\Gamma$-covering of $\Sigma_{n}$, and $X \equiv \Gamma \backslash\left(\widetilde{\Sigma}_{n}^{\prime} \times Z\right)$. 
We can use suspension foliated spaces to easily produce examples of transitive foliated spaces whose leaves have different asymptotic dimension. A very simple one is given by any suspension of an action of $\mathbb{Z}^{2}$ on the circle, where one point is fixed, and the other points have trivial isotropy groups and dense orbits. We get one compact leaf, which has asymptotic dimension zero, and the other leaves are coarsely quasi-isometric to $\mathbb{Z}^{2}$, which has asymptotic dimension two [16].

\subsection{Foliated spaces defined by locally free actions of Lie groups}

Any locally free action of a connected Lie group $G$ on a Polish space $X$ defines a foliated structure on $X$ whose leaves are the orbits [23, Theorem 11.3.9]. Many interesting examples of this type are given in [23, Chapter 11]. If we equip $G$ with a right invariant Riemannian metric, then the leaf through every $x \in X$, $G \cdot x \equiv G / G_{x}$, can be equipped with the induced metric, obtaining that $X$ is a compact Riemannian foliated space. The isotropy groups $G_{x}$ are discrete in $G$. If they are trivial, then all leaves are isometric to $G$.

Assume that $X$ is compact. In general, two leaves are coarsely-quasi-isometric if the corresponding conjugacy classes of isotropy groups have commensurable representatives; again, the commensurability up to conjugacy refines the coarse quasiisometry relation.

\subsection{Inverse limits of covering spaces}

A compact connected foliated space whose local trasversals are totally disconnected is called a matchbox manifold. The following is a typical construction of matchbox manifolds.

Let $M_{0} \leftarrow M_{1} \leftarrow \cdots$ be a tower of smooth non-trivial finite fold regular covering maps between closed smooth manifolds. Its inverse limit $X$ is a compact minimal smooth matchbox manifold, called McCord or regular solenoid, which generalizes the usual solenoid, where every $M_{i}$ is the circle. McCord solenoids are just the homogeneous smooth matchbox manifolds, except for closed manifolds [34, Theorem 1.2]. By definition of homogeneity, all leaves of $X$ are coarsely quasi-isometric to each other (the alternative (i) of Theorem 1.2). The groups $\Gamma_{i}=\pi_{1}\left(M_{i}\right)$ form a nested sequence of normal subgroups, $\Gamma_{0} \triangleright \Gamma_{1} \triangleright \ldots$, and the groups of deck transformations of the composites $M_{0} \leftarrow M_{i}$ form a sequence of homomorphisms between finite groups, $\{1\} \leftarrow \Gamma_{0} / \Gamma_{1} \leftarrow \Gamma_{0} / \Gamma_{2} \leftarrow \cdots$, whose inverse limit is a topological group $Z$ with a canonical action of $\Gamma_{0}$. Then $X$ can be identified to the suspension of this action, using the universal covering $\widetilde{M} \rightarrow M$. The underlying space of $Z$ is a Cantor space.

If the covering maps are not required to be regular, then the term weak solenoid is used. The weak solenoids are just the equicontinuous matchbox manifolds, except for closed manifolds [34, Theorem 1.4]. In this case, the Cantor space $Z$ has no induced group structure because the sets $\Gamma_{0} / \Gamma_{i}$ may not be groups. But we continue having an action of $\Gamma_{0}$ on $Z$, realizing $X$ as suspension. Under some additional conditions, the holonomy covers of all leaves are coarsely quasi-isometric to each other by equicontinuity [10, Theorem 17.3].

More general matchbox manifolds can be described with inverse limits if we allow branched coverings [2, [76]. 


\subsection{Bounded geometry and leaves}

Let $M=(M, g)$ be complete connected Riemannian manifold. As usual, let $\nabla$ denote its Levi-Civita connection, and $\operatorname{Iso}(M)$ its group of isometries. It is said that $M$ is non-periodic (respectively, locally non-periodic) if $\operatorname{Iso}(M)=\left\{\operatorname{id}_{M}\right\}$ (respectively, the canonical projection $M \rightarrow \operatorname{Iso}(M) \backslash M$ is a covering map). For any domain $\Omega$ in $M$ and every smooth tensor $T$ on $\Omega$, let $\|T\|_{\Omega}=\sup _{\Omega}|T|$. It is said that $M$ is limit-aperiodic if, for all sequences, $m_{i} \uparrow \infty$ in $\mathbb{N}$, of compact domains $\Omega_{i}^{\prime} \subset \Omega_{i} \subset M$, of points $x_{i} \in \Omega_{i}^{\prime}$ and $y_{i} \in \Omega_{i}$, and of $C^{m_{i}}$ pointed embeddings $\phi_{i j}:\left(\Omega_{i}, x_{i}\right) \rightarrow\left(\Omega_{j}, x_{j}\right)(i \leq j)$ and $\psi_{i}:\left(\Omega_{i}^{\prime}, x_{i}\right) \rightarrow\left(\Omega_{i}, y_{i}\right)$, such that

$$
\left.\lim _{i} d\left(x_{i}, \partial \Omega_{i}^{\prime}\right)\right)=\infty, \quad \lim _{i, j}\left\|\nabla^{m_{i}}\left(g-\phi_{i j}^{*} g\right)\right\|_{\Omega_{i}}=\lim _{i}\left\|\nabla^{m_{i}}\left(g-\psi_{i}^{*} g\right)\right\|_{\Omega_{i}^{\prime}}=0,
$$

we have

$$
\lim _{i} \max \left\{d\left(x, \psi_{i}(x)\right) \mid x \in \Omega_{i}^{\prime} \cap \bar{B}\left(x_{i}, r\right)\right\}=0
$$

for some $r>0$ [7, Definition 12.4]. Finally, it is said that $M$ is repetitive if, for every compact domain $\Omega$ in $M$, and all $\varepsilon>0$ and $m \in \mathbb{N}$, there is a family of $C^{m}$ embeddings $\phi_{i}: \Omega \rightarrow M$ such that $\bigcup_{i} \phi_{i}(\Omega)$ is a net in $M$ and $\left\|\nabla^{m}\left(g-\phi_{i}^{*} g\right)\right\|_{\Omega}<\varepsilon$ for all $i$ [7, Definition 12.6].

It is obvious that any leaf $L$ of a compact Riemannian foliated space $X$ is of bounded geometry. If moreover $L$ is without holonomy and $X$ minimal, then $L$ is repetitive, which is a direct consequence of the local Reeb stability [23, Proposition 11.4.8]. The converse statements are given by the following result.

Theorem 11.1 ([5]; see also [7, Theorem 1.5], [6. Theorem 1.1]). Any (repetitive) Riemannian manifold $M$ of bounded geometry can be realized as a leaf of a (minimal) compact Riemannian foliated space $X$ without holonomy.

Thus the general study the leaves of (minimal) compact Riemannian foliated spaces without holonomy is the study of (repetitive) Riemannian manifolds of bounded geometry.

Let us recall the construction of $X$ in Theorem 11.1 because it is a source of examples of compact foliated spaces with prescribed leaves. To begin with, we recall a simpler construction that only works under additional conditions on the manifold. For any $n \in \mathbb{N}$, let $\mathcal{M}_{*}(n)$ denote the set $t^{1}$ of isometry classes, $[M, x]$, of pointed complete connected Riemannian $n$-manifolds, $(M, x)$. A sequence $\left[M_{i}, x_{i}\right] \in \mathcal{M}_{*}(n)$ is said to be $C^{\infty}$ convergent to $[M, x] \in \mathcal{M}_{*}(n)$ if, for every compact domain $\Omega \subset M$ containing $x$, there are pointed $C^{\infty}$ embeddings $\phi_{i}:(\Omega, x) \rightarrow\left(M_{i}, x_{i}\right)$ for large enough $i$ such that

$$
\lim _{i}\left\|\nabla^{m}\left(\phi_{i}^{*} g_{i}-g\right)\right\|_{\Omega}=0
$$

for all $m \in \mathbb{N}$ [81, Chapter 10, Section 3.2]. This convergence on $\mathcal{M}_{*}(n)$ defines a Polish topology [7, Theorem 1.2], [17, Appendix A] (see also [81, Chapter 10], [73]). The corresponding Polish space is denoted by $\mathcal{M}_{\star}^{\infty}(n)$, and its closure operator by $\mathrm{Cl}_{\infty}$. There is a continuous injection of $\mathcal{M}_{*}^{\infty}(n)$ into the Gromov space $\mathcal{M}_{*}$ of isometry classes of pointed proper metric spaces [54, [56, Chapter 3]. For every complete connected Riemannian $n$-manifold $M$, there is a canonical continuous map $\iota_{M}: M \rightarrow \mathcal{M}_{*}^{\infty}(n)$, given by $\iota_{M}(x)=[M, x]$, which induces a continuous injection $\bar{\iota}_{M}$ : Iso $(M) \backslash M \rightarrow \mathcal{M}_{*}^{\infty}(n)$. The images of all possible maps $\iota_{M}$ form a

\footnotetext{
${ }^{1}$ This set is well defined by assuming that the underlying set of every $M$ is contained in a common set.
} 
partition $\mathcal{F}_{*}(n)$ of $\mathcal{M}_{*}^{\infty}(n)$; i.e., every set of this partition is defined by varying the distinguished point in a fixed Riemannian manifold. The non-periodic and locally non-periodic manifolds define subspaces $\mathcal{M}_{*, \text { np }}^{\infty}(n) \subset \mathcal{M}_{*, \text { nnp }}^{\infty}(n) \subset \mathcal{M}_{*}^{\infty}(n)$, and let $\mathcal{F}_{*, \operatorname{lnp}}(n)$ denote the restriction of $\mathcal{F}_{*}(n)$ to $\mathcal{M}_{*, \operatorname{lnp}}(n)$. Assume $n \geq 2$, otherwise $\mathcal{M}_{*}^{\infty}(n)$ is too simple. Then $\mathcal{M}_{*, \operatorname{lnp}}^{\infty}(n)$ is open and dense in $\mathcal{M}_{*}^{\infty}(n)$, $\mathcal{F}_{*, \operatorname{lnp}}(n)$ underlies a canonical $C^{\infty}$ foliated structure $\mathcal{F}_{*, \operatorname{lnp}}^{\infty}(n)$, and the $C^{\infty}$ foliated space $\mathcal{M}_{*, \operatorname{lnp}}^{\infty}(n) \equiv\left(\mathcal{M}_{*, \operatorname{lnp}}^{\infty}(n), \mathcal{F}_{* \text {,np }}^{\infty}(n)\right)$ has a canonical Riemannian structure [7. Theorem 1.3]. Moreover the holonomy covers of the leaves are of the form $\iota_{M}: M \rightarrow \operatorname{im} \iota_{M}$, which are local isometries. Thus the union of leaves without holonomy is the subspace $\mathcal{M}_{*, \mathrm{np}}^{\infty}(n)$. On the other hand, $\mathrm{Cl}_{\infty}\left(\mathrm{im} \iota_{M}\right)$ is compact if and only if $M$ is of bounded geometry [7, Theorem 12.3] (see also [33, 81, Chapter 10, Sections 3 and 4]), and $M$ is limit-aperiodic (respectively, repetitive) if and only if $\mathrm{Cl}_{\infty}\left(\mathrm{im} \iota_{M}\right) \subset \mathcal{M}_{* \text { np }}^{\infty}(n)$ (respectively, $\mathrm{Cl}_{\infty}\left(\mathrm{im} \iota_{M}\right)$ is minimal) 7 , Lemmas 12.5 and 12.7]. Thus, if $M$ is of bounded geometry and limit-aperiodic, then it is isometric to a leaf of the compact Riemannian foliated space without holonomy, $\mathrm{Cl}_{\infty}\left(\mathrm{im} \iota_{M}\right)$, which is minimal if $M$ is also repetitive.

To avoid the requirement of limit-aperiodicity of $M$, this condition is achieved by adding extra structure on $M$, given by a distinguished function. Precisely, fix a separable Hilbert space $\mathbb{E}$, and consider pairs $(M, f)$, where $f \in C^{\infty}(M, \mathbb{E})$, instead of just the simply connected Riemannian $n$-manifold $M$. An isomorphism of these objects is an isometry compatible with the distinguished functions. Then, proceeding as above, equivalence classes $[M, f, x]$ can be defined by using pointed isomorphisms. They form a set $\widehat{\mathcal{M}}_{*}(n)$, where there is an obvious version of the $C^{\infty}$ convergence. This convergence defines a Polish space $\widehat{\mathcal{M}}_{*}^{\infty}(n)$ [6. Theorem 1.3], whose closure operator is denoted by $\widehat{\mathrm{Cl}}_{\infty}$. There are also canonical maps $\hat{\iota}_{M, f}$ : $M \rightarrow \widehat{\mathcal{M}}_{*}(n)$, whose images form a natural partition $\widehat{\mathcal{F}}_{*}(n)$. The concepts of being non-periodic, locally non-periodic, limit-aperiodic or repetitive have obvious versions for pairs $(M, f)$ (or simply for $f$ ), obtaining $\widehat{\mathcal{M}}_{*, \text { np }}^{\infty}(n)$ and $\widehat{\mathcal{M}}_{*, \operatorname{lnp}}^{\infty}(n) \equiv$ $\left(\widehat{\mathcal{M}}_{*, \operatorname{lnp}}^{\infty}(n), \widehat{\mathcal{F}}_{*, \operatorname{lnp}}^{\infty}(n)\right)$ as above, satisfying analogous properties (without requiring $n \geq 2)$ [6. Section 1]; in particular, $\widehat{\mathcal{M}}_{*, \operatorname{lnp}}^{\infty}(n)$ is a Riemannian foliated space, whose subspace of leaves without holonomy is $\widehat{\mathcal{M}}_{*, \mathrm{np}}^{\infty}(n)$. This foliated space is universal among Riemannian foliated spaces satisifying a property called covering-continuity 6. Proposition 6.4]. Moreover $(M, f)$ (or simply $f$ ) is said to be of bounded geometry if $M$ is of bounded geometry and $\left\|\nabla^{m} f\right\|_{M}<\infty$ for all $m \in \mathbb{N}$. This property means that $\widehat{\mathrm{Cl}}_{\infty}\left(\operatorname{im} \hat{\iota}_{M, f}\right)$ is compact [6, Claim 7.4]. Then Theorem 11.1 follows with $X=\widehat{\mathrm{Cl}}{ }_{\infty}\left(\operatorname{im} \hat{\iota}_{M, f}\right)$, where $f$ is given by the following result.

Proposition 11.2 (Álvarez-Barral [5, see also [6 Proposition 7.1]). For any (repetitive) connected Riemannian manifold $M$ of bounded geometry, there is some (repetitive) limit-aperiodic function $f \in C^{\infty}(M, \mathbb{E})$ of bounded geometry.

The construction of $f$ in Proposition 11.2 will be indicated in Section 11.7. It will be reduced to a graph version, which is indicated first in Section 11.5

For instance, Theorem 11.1 can be applied to any complete connected hyperbolic manifold with a positive injectivity radius. It can be also applied to any connected Lie group with a left invariant metric. Some of them are not coarsely quasi-isometric to (the Cayley graph of) any finitely generated group [32, 43, obtaining compact minimal Riemannian foliated spaces without holonomy, whose 
leaves are isometric to each other, but the leaves are not coarsely quasi-isometric to any finitely generated group.

The results of Chapter 1 can be illustrated by applying Theorem 11.1 to appropriate Riemannian manifolds. But it is simpler to construct graphs of finite type and the required properties. Then we can consider the corresponding subspaces in $\mathcal{G}_{*}$, or in any of its variants, which can be transformed into foliated spaces by a standard procedure, called taking the boundary of a thickening. This will produce compact foliated spaces with all possible quasi-isometric types of leaves according to Proposition 3.25. This way of constructing examples is explained in Section 11.8 . and concrete examples are given in Section 11.9 .

\subsection{Graph spaces}

We will only consider connected graphs $G$ with a countable set of vertices, all of them with finite degree. Moreover, unless otherwise stated, the graphs are simple in the sense that there are no loop edges, there is at most one edge between any pair of vertices, and the edges have no orientations. Identify $G$ with its vertex set, $G \equiv V(G)$, and let $E(G)$ denote its edge set. With the canonical graph metric, these vertex sets are proper metric spaces. Since a graph isomorphism is the same as an isometry between graphs, the pointed isomorfism classes of pointed graphs also form a subspace $\mathcal{G}_{*}$ of the Gromov space $\mathcal{M}_{*}$. Precisely, a local base at any $[G, v] \in \mathcal{G}_{*}$ is given by the sets

$$
\mathcal{U}_{G, v, R}=\left\{\left[G^{\prime}, v^{\prime}\right] \in \mathcal{G}_{*} \mid\left(\bar{B}_{G^{\prime}}\left(v^{\prime}, R\right), v^{\prime}\right) \cong\left(\bar{B}_{G}(v, R), v\right)\right\} \quad(R>0),
$$

where isomorphisms of pointed graphs are used. A compatible ultra-metric $d_{\mathcal{G}_{*}}$ on $\mathcal{G}_{*}$ can be defined by

$$
d_{\mathcal{G}_{*}}\left([G, v],\left[G^{\prime}, v^{\prime}\right]\right)=\exp \left(-\sup \left\{R>0 \mid\left[G^{\prime}, v^{\prime}\right] \in \mathcal{U}_{G, v, R}\right\}\right) .
$$

Note that $\mathcal{G}_{*}$ is totally disconnected. Let $\mathrm{Cl}$ denote the closure operator in $\mathcal{G}_{*}$. For every graph $G$, there is a canonical map $\iota_{G}: G \rightarrow \mathcal{G}_{*}$, defined like $\iota_{M}$ in Section 11.4, obtaining a transitive partition of $\mathcal{G}_{*}$ into graphs with possible loop edges (the image of every $\iota_{G}$ is a quotient graph of $G$ that may have loop edges). Precisely, two elements $\mathbf{z}, \mathbf{z}^{\prime} \in \mathcal{G}_{*}$ are called contiguous if $\mathbf{z}=[G, v]$ and $\mathbf{z}^{\prime}=\left[G, z^{\prime}\right]$ for some contiguous vertices $v$ and $v^{\prime}$ in some graph $G$. The relation of contiguity on $\mathcal{G}_{*}$ is also independent of the representatives. Thus $\mathcal{G}_{*}$ is kind of a space foliated by graphs. Any transitive saturated subspace $Z=\mathrm{Cl}\left(\operatorname{im} \iota_{G}\right) \subset \mathcal{G}_{*}$ can be called a graph space. This $Z$ is compact if and only if $G$ is of finite type.

Other versions of graph spaces can be considered as well, like a version of $\widehat{\mathcal{M}}_{*}(n)$ in Section 11.4. To begin with, take (vertex) colorings of graphs with values in $\mathbb{N}$. This gives rise to the totally disconnected space $\widehat{\mathcal{G}}_{*}$ of isomorphism classes of pointed colored graphs, where a local base at every $[G, \alpha, v] \in \widehat{\mathcal{G}}_{*}$ is given by the sets $\widehat{\mathcal{U}}_{G, \alpha, v, R}(R>0)$, defined like in 111.1 by using pointed colored graphs isomorphisms. Let $\widehat{\mathrm{Cl}}$ denote the closure operator in $\widehat{\mathcal{G}}_{*}$. For every colored graph $(G, \alpha)$, there is a canonical map $\hat{\iota}_{G, \alpha}: G \rightarrow \widehat{\mathcal{G}}_{*}$, defined like $\hat{\iota}_{M, f}$ in Section 11.4 . obtaining a canonical transitive partition of $\widehat{\mathcal{G}}_{*}$ into colored graphs with possible loop edges.

Now, take edge-colored graphs, $(G, \beta)$, where $\beta: E(G) \rightarrow \mathbb{N}$. They define a totally disconnected space $\mathcal{G}_{*}^{\prime}$, where a local base at every $[G, \beta, v] \in \mathcal{G}_{*}^{\prime}$ is given by the sets $\mathcal{U}_{G, \alpha, v, R}^{\prime}(R>0)$, defined like in 11.1 by using pointed edge-colored 
graph isomorphisms. A compatible metric can be defined like in (11.2). This space is equipped with a canonical transitive partition defined by the images of canonical maps $\iota_{G, \beta}^{\prime}: G \rightarrow \mathcal{G}_{*}^{\prime}$. The closure operator of $\mathcal{G}_{*}^{\prime}$ is denoted by $\mathrm{Cl}^{\prime}$.

Consider also partially directed graphs, $(G, \mathcal{O})$, where the partial direction $\mathcal{O}$ assigns an orientation ${ }^{2}$ to the edges in some subset of $E(G)$. Their directionpreserving pointed isomorphism classes form a totally disconnected space $\mathcal{G}_{*,+}$, where a local base at every $[G, \mathcal{O}, v] \in \mathcal{G}_{*,+}$ consists of the sets $\mathcal{U}_{G, \mathcal{O}, v, R}(R>0)$, defined like in (11.1) by using pointed direction-preserving graph isomorphisms. This space is equipped with a canonical transitive partition into the images of canonical maps $\iota_{G, \mathcal{O}}: G \rightarrow \mathcal{G}_{*,+}$. The closure operator of $\mathcal{G}_{*,+}$ is denoted by $\mathrm{Cl}_{+}$. Note that $\mathcal{G}_{*}$ is the saturated subspace of $\mathcal{G}_{*,+}$ defined by the graphs with empty partial orientation.

We can also combine several of the above structures on graphs in an obvious way, giving rise to the totally disconnected spaces $\widehat{\mathcal{G}}_{*}^{\prime}, \widehat{\mathcal{G}}_{*,+}, \mathcal{G}_{*,+}^{\prime}$ and $\widehat{\mathcal{G}}_{*,+}^{\prime}$, where the closure operators are denoted by $\widehat{\mathrm{Cl}}_{*}^{\prime}, \widehat{\mathrm{Cl}}_{+}, \mathrm{Cl}_{+}^{\prime}$ and $\widehat{\mathrm{Cl}}_{+}^{\prime}$, and with canonical maps, $\hat{\iota}_{G, \alpha, \beta}: V(G) \rightarrow \widehat{\mathcal{G}}_{*}^{\prime}, \hat{\iota}_{G, \alpha, \mathcal{O}}: G \rightarrow \widehat{\mathcal{G}}_{*,+}, \iota_{G, \beta, \mathcal{O}}^{\prime}: G \rightarrow \mathcal{G}_{*,+}^{\prime}$ and $\hat{\iota}_{G, \alpha, \beta, \mathcal{O}}^{\prime}: G \rightarrow \widehat{\mathcal{G}}_{*,+}^{\prime}$, whose images define canonical transitive partitions of these spaces.

The concepts of being non-periodic, locally non-periodic, limit-aperiodic and repetitive have obvious versions for graphs with possible additional structures of the above type, using graph isomorphisms preserving those structures. The concept of bounded geometry is played by the condition of finite type in the case of graphs, with the additional condition of using finitely many colors in the case of vertex or edge colorings. Thus the following is a version of Proposition 11.2 for graphs.

Theorem 11.3 (Álvarez-Barral [5]). If a (repetitive) connected graph $G$ has vertex degrees uniformly bounded by some $c \in \mathbb{N}$, then $G$ has a (repetitive) limitaperiodic coloring by c colors.

In Theorem 11.3, the number of colors is optimal with this generality (consider the Cayley graph of $\mathbb{Z}$, or any complete finite graph). But indeed the existence of a (repetitive) limit-aperiodic coloring by finitely many colors would be enough for our purposes. The proof of Theorem 11.3 is very involved to achieve the optimal number of colors. It would be much simpler if only any finite number of colors is required. There is a version of Theorem 11.3 for edge colorings, which indeed can be obtained as a corollary [5].

\subsection{Case of Cayley graphs}

As an example of Section 11.5, consider the Cayley graph $G(\Gamma, S)$ of any finitely generated group $\Gamma$ and a finite set of generators $S$, defined so that right translations are graph isomorphisms (Section 3.1. Example 3.1). We also use $\Gamma$ to denote this graph. We can assume that $S \cap S^{-1}$ consists only of elements of order two, different from the identity. Then $\Gamma$ can be equipped with a right invariant $S$-valued edge coloring $\beta$ and a right invariant partial orientation $\mathcal{O}$, defined as follows. For an edge $e$ joining $v, w \in \Gamma$ :

- let $\beta(e)=a \in S$ if $a v=w$ or $a w=v$;

- declare that $e \in \operatorname{dom} \mathcal{O}$ just when $a$ is not of order two; and,

\footnotetext{
${ }^{2}$ Recall that an orientation of an edge can be understood as an order of its vertices, which can be written as an ordered pair of its vertices.
} 
- in this case, define $\mathcal{O}(e)=(v, w)$ if $a v=w$, and $\mathcal{O}(e)=(w, v)$ if $a w=v$.

REMARK 11.4. Note that the edges $e$ with a common vertex $v$ are determined by $\beta(e)$ and the position of $v$ in $\mathcal{O}(e)$, if $e \in \operatorname{dom} \mathcal{O}$. This property is also satisfied by any connected subgraph equipped with the restrictions of $\beta$ and $\mathcal{O}$.

The above edge coloring and partial orientation will be always considered on $\Gamma$, often without mentioning them. After choosing an injective map $S \rightarrow \mathbb{N}$ to consider $\beta$ with values in $\mathbb{N}$, we get a compact saturated subspace $\mathrm{Cl}_{+}^{\prime}\left(\operatorname{im}_{\Gamma, \beta, \mathcal{O}}\right) \subset \mathcal{G}_{*,+}^{\prime}$. We may also consider the compact subspace of $\mathcal{G}_{*,+}^{\prime}$ defined by all connected subgraphs of $\Gamma$, or by a class of those subgraphs, like trees. And we may also add vertex colorings in any of these constructions.

However, when dealing with connected subgraphs of $\Gamma$ and their vertex or edge colorings, it is interesting to modify the previous definitions by using only right translations instead of arbitrary isomorphisms. To begin with, the definitions of being non-periodic, limit-aperiodic or repetitive are modified by using this restriction on the type of isomorphisms; the terms $\Gamma$-non-periodic, $\Gamma$-limit-aperiodic and $\Gamma$-repetitive will be used for these versions. Being $\Gamma$-non-periodic or $\Gamma$-limitaperiodic is weaker than being non-periodic or limit-aperiodic, respectively, and being $\Gamma$-repetitive is stronger than being repetitive.

The following space, defined with the above point of view, is very practical to construct concrete examples. Let $\mathcal{T}=\mathcal{T}(\Gamma)$ be the compact totally disconnected space of pointed trees in $\Gamma$, up to right translations, with the topology described by local bases defined like in (11.1), using only isomorphisms between balls given by right translations [51, [18, [74, 75, 1], 78, and a corresponding ultrametric can be defined like in (11.2). This definition can be simplified because, after using right translations, the distinguished point can be chosen to be the identity element 1 , which can be omitted from the terminology and notation. Thus $\mathcal{T}$ can be described as the space of trees $T$ in $\Gamma$ containing 1 , and its topology can be described by a local base at every $T$ consisting of the sets

$$
\mathcal{U}_{T, R}=\left\{T^{\prime} \in \mathcal{T} \mid \bar{B}_{T}(1, R)=\bar{B}_{T^{\prime}}(1, R)\right\} \quad(R>0) .
$$

Again, a compatible ultra-metric can be defined using the sets $\mathcal{U}_{T, R}$ like in 11.2 . Now, in the canonical transitive partition of $\mathcal{T}$, the class of every $T \in \mathcal{T}$ is $\left\{T \gamma^{-1} \mid\right.$ $\gamma \in T\}$, which can be identified with $T$ using the mapping $T \gamma^{-1} \mapsto \gamma$. The graph structure, edge coloring and partial direction of $T$ passes to the corresponding set of the canonical partition via this identity. The right action of $\Gamma$ on itself induces a compactly generated pseudogroup $\mathcal{H}$ on $\mathcal{T}$ : for $T \in \mathcal{T}$ and $\gamma \in \Gamma, T$ is in the domain of the map in $\mathcal{H}$ defined by $\gamma$ just when $T \cdot \gamma \in \mathcal{T}$; i.e., $\gamma^{-1} \in T$.

If $S$ does not contain elements of order two, then any tree in $\mathcal{T}(\Gamma)$ can be obviously considered as a tree in the free group $F_{n}$ with $n=|S|$ generators, obtaining a canonical embedding of $\mathcal{T}(\Gamma)$ into $\mathcal{T}\left(F_{n}\right)$, which is compatible with the corresponding pseudogroups, and canonical edge colorings and orientations (which are global in this case). Thus considering $\mathcal{T}\left(F_{n}\right)$ is enough in many cases.

We will also use the obvious version $\widehat{\mathcal{T}}(c)=\widehat{\mathcal{T}}(\Gamma, c)$ of $\mathcal{T}$, which consists of subtrees of $\Gamma$ containing 1 equipped with vertex colorings by colors in $\{0,1, \ldots, c\}$ $\left(c \in \mathbb{Z}^{+}\right)$. It satisfies analogous properties.

Similarly, we can fix the graph $\Gamma$ and take arbitrary vertex colorings by colors in the set $\{0, \ldots, c\}$, defining the compact coloring space $\{0, \ldots, c\}$, with the Tychonoff topology, and a compatible ultra-metric defined like in $(11.2)$. There is no 
need to indicate the fixed point because it can be assumed to be 1 as before. Thus the canonical transitive partition is now given by the orbits of the transitive left action of $\Gamma$ on $\{0, \ldots, c\}^{\Gamma}$ (induced by the right action of $\Gamma$ on itself by right translations). This coloring space with this action is called a Bernouilli shift. For any coloring $\alpha \in\{0, \ldots, c\}^{\Gamma}$, its orbit closure $\overline{\Gamma \cdot \alpha}$ with the restriction of the $\Gamma$-action is called a subshift. The left action of $\Gamma$ on $\overline{\Gamma \cdot \alpha}$ is free (respectively, minimal) just when $\alpha$ is $\Gamma$-limit-aperiodic (respectively, $\Gamma$-repetitive). Since the graph structure, edge coloring and partial direction of $\Gamma$ are right invariant, they can be projected to $\Gamma / \Gamma_{\alpha} \equiv \Gamma \cdot \alpha$. There always exist some $\Gamma$-limit-aperiodic coloring $\alpha$ by two colors, which indeed holds for any countable group [46, [13. Moreover we can assume that this $\alpha$ is repetitive, otherwise we can find a repetitive coloring in a minimal set of $\overline{\Gamma \cdot \alpha}$. Thus we will consider only the Bernouilli shift $\{0,1\}^{\Gamma}$.

Bernuilli shifts play an important role in Dynamics. For instance, they are expansive, and indeed universally expansive: any expansive $\Gamma$-action on a compact metric space is an equivariant quotient of some closed invariant subspace of $\{0, \ldots, c\}^{\Gamma}$, for $c$ large enough, and this quotient map is an equivariant homeomorphism in the totally disconnected case [35, Proposition 2.6].

There is an orbit equivalent embedding of any Bernouilli shift $\{0, \ldots, c\}^{\Gamma}$ into $\mathcal{T}\left(F_{n}\right)$ for $n$ large enough [77, Theorem 1.1]. But this embedding is non-canonical and rather involved.

With more generality, we can consider a semigroup $\Gamma$, like $\mathbb{N}$. Then the Bernouilli shift is given by the induced semigroup action of $\Gamma$ on $\{0, \ldots, c\}^{\Gamma}$. The Bernouilli shifts defined by semigroups are used in the study automatic sequences (see e.g. [4] [80, 82]). In the case of $\mathbb{N}$, the colorings in $\{0, \ldots, c\}^{\mathbb{N}}$ can be considered as infinite words using the alphabet $\{0, \ldots, c\}$; for instance, the Fibonacci and Thue-Morse words in $\{0,1\}^{\mathbb{N}}$ are very relevant.

\subsection{Construction of limit-aperiodic functions}

Let us indicate the proof of Proposition 11.2 using Theorem 11.3 By the bounded geometry of $M$, there is some $0<r<\operatorname{inj}_{M}$ such that the following properties hold:

(i) For the normal parametrizations $\kappa_{x}: B_{r}:=B_{\mathbb{R}^{n}}(0, r) \rightarrow B_{M}(x, r)(x \in M)$, the corresponding metric coefficients, $g_{i j}$ and $g^{i j}$, as a family of $C^{\infty}$ functions on $B_{r}$ parametrized by $x, i$ and $j$, lie in a bounded subset of the Fréchet space $C^{\infty}\left(B_{r}\right)$ [88, Theorem A.1], [89, Theorem 2.5] (see also [83, Proposition 2.4], [41).

(ii) There is some countable subset $\left\{x_{i} \mid i \in I\right\} \subset M$ and some $c \in \mathbb{N}$ such that the balls $B_{M}\left(x_{i}, r / 2\right)$ cover $M$, and, for all $x \in M, B_{M}(x, r)$ meets at most $c$ balls $B_{M}\left(x_{i}, r\right)$ [93, A1.2 and A1.3], 89, Proposition 3.2]. Let $\kappa_{i}=\kappa_{x_{i}}$.

Consider the graph $G$ with $V(G)=I$, and such that there is an edge connecting two different vertices, $i$ and $j$, if and only if $B_{M}\left(x_{i}, r\right) \cap B_{M}\left(x_{j}, r\right) \neq \varnothing$. By (ii), the vertex degrees are uniformly bounded by $c$. So there is a coloring of $G$ by $c+1$ colors so that adjacent vertices have different colors. This means that there is a partition of $I$ into finitely many sets, $I_{1}, \ldots, I_{c+1}$, such that $B_{M}\left(x_{i}, r\right) \cap B_{M}\left(x_{j}, r\right)=\varnothing$ for $i \in I_{k}$ and $j \in I_{l}$ with $k \neq l$. On the other hand, by Theorem 11.3, $G$ has a limitaperiodic vertex coloring $\alpha: I \rightarrow\{1, \ldots, c\}$. Let $\alpha_{i}=\alpha\left(x_{i}\right)$. 
Let $S$ be an isometric copy in $\mathbb{R}^{n+1}$ of the standard $n$-dimensional sphere so that $0 \in S$. Choose some radia ${ }^{3}$ function $\rho \in C^{\infty}\left(\mathbb{R}^{n}\right)$ such that $0 \leq \rho \leq 1, \rho(x)=1$ if $|x| \leq r / 2$ and $\rho(x)=0$ if $|x| \geq r$. Take also some $C^{\infty}$ map $\tau: \mathbb{R}^{n} \rightarrow \mathbb{R}^{n+1}$ that restricts to a diffeomorphism $B_{r} \rightarrow S \backslash\{0\}$ and maps $\mathbb{R}^{n} \backslash B_{r}$ to 0 . Let $\rho_{i}=\rho \circ \kappa_{i}^{-1}$ and $\tau_{i}=\tau \circ \kappa_{i}^{-1}$. For $k=1, \ldots, c+1$, define $f^{k}: M \rightarrow \mathbb{R}^{n+2}$ by

$$
f^{k}(x)= \begin{cases}0 & \text { if } x \notin \bigcup_{i \in I_{k}} B_{M}\left(x_{i}, r\right) \\ \left(\rho_{i}(x) \cdot \alpha_{i}, \rho_{i}(x) \cdot \tau_{i}(x)\right) & \text { if } x \in B_{M}\left(x_{i}, r\right) \text { for some } i \in I_{k} .\end{cases}
$$

Fix a linear injection $\mathbb{R}^{(c+1)(n+2)} \subset \mathbb{E}$. Then

$$
f=\left(f^{1}, \ldots, f^{c+1}\right): M \rightarrow \mathbb{R}^{(c+1)(n+2)} \subset \mathbb{E}
$$

is a $C^{\infty}$ immersion, and therefore it is locally non-periodic. Moreover $f$ is of bounded geometry and limit-aperiodic, as follows from (i), and from the bounded geometry and limit-aperiodicity of $\alpha$.

If $M$ is repetitive, then this property can be easily used to choose the points $x_{i}$ so that the pair $\left(M,\left\{x_{i}\right\}\right)$ is repetitive in an obvious sense (as a Riemannian manifold with a distinguished subset). With this condition, $G$ is repetitive, and $\alpha$ can be assumed to be repetitive by Theorem 11.3 It follows that $f$ is also repetitive, showing Proposition 11.2

Smaller subspaces, $\widehat{\mathcal{M}}_{* \text {,imm }}^{\infty}(n) \subset \widehat{\mathcal{M}}_{*, \text { Inp }}^{\infty}(n)$ and $\widehat{\mathcal{M}}_{* \text { emb }}^{\infty}(n) \subset \widehat{\mathcal{M}}_{*, \text { np }}^{\infty}(n)$, are defined by requiring the distinguished functions to be $C^{\infty}$ immersions or $C^{\infty}$ embeddings. It turns out that $\widehat{\mathcal{M}}_{*, \text { imm }}^{\infty}(n)$ is Polish and dense in $\widehat{\mathcal{M}}_{*}^{\infty}(n)[\mathbf{6}$, Theorem 1.4]. Thus we get a $C^{\infty}$ and Riemannian foliated subspace, $\widehat{\mathcal{M}}_{*, \text { imm }}(n) \equiv$ $\left(\widehat{\mathcal{M}}_{* \text {,imm }}^{\infty}(n), \widehat{\mathcal{F}}_{* \text {,imm }}^{\infty}(n)\right)$, where $\widehat{\mathcal{M}}_{*, \text { emb }}^{\infty}(n)$ is a union of leaves without holonomy. In fact, we can use the distinguished immersions to define its foliated charts more easily [6, Theorem 1.4]. Then there is some $h \in C^{\infty}(M, \mathbb{E})$ such that $\widehat{\mathrm{Cl}}_{\infty}\left(\operatorname{im} \hat{\iota}_{M, h}\right)$ is a (minimal) compact subspace of $\widehat{\mathcal{M}}_{*, \mathrm{emb}}^{\infty}(n)$. This slight sharpening of Proposition 11.2 can be easily proved as follows. Let $f \in C^{\infty}(M, \mathbb{E})$ be given by Proposition 11.2 inducing the foliated space $X=\widehat{\mathrm{Cl}}_{\infty}\left(\operatorname{im} \hat{\iota}_{M, f}\right)$. Then there is a $C^{\infty}$ embedding $\tilde{h}: X \rightarrow \mathbb{E}\left[\mathbf{2 3}\right.$, Theorem 11.4.4], and the function $h=\hat{\iota}_{M, f}^{*} \tilde{h} \in C^{\infty}(M, \mathbb{E})$ satisfies the above property. However $\widehat{\mathrm{Cl}}_{\infty}\left(\operatorname{im} \hat{\iota}_{M, h}\right) \equiv X$ (no new foliated space is produced with this sharpening).

Distinguished subsets of Riemannian manifolds can be used instead of distinguished functions to construct a Riemannian foliated space, producing also compact Riemannian foliated spaces with a prescribed leaf $[\mathbf{1 7}$.

\subsection{Graph matchbox manifolds}

We explain the method of taking the boundary of a thickening, which transforms graph spaces into Riemannian matchbox manifolds [51, 18, 74, 75, 1], 78, 77.

Given $c \in \mathbb{N}$, for every $i=0, \ldots, c$, let $\Sigma_{i}$ be the compact orientable connected surface of genus zero and $i$ boundary components; i.e., $\Sigma_{i}$ is the 2 -sphere with $i$ disjoint open disks taken out. For any subset $Q \subset\{1, \ldots, c\} \times\{0, \pm 1\}$ with $i$ elements, fix a bijective map $\sigma_{Q}: \pi_{0}\left(\partial \Sigma_{i}\right) \rightarrow Q$. Choose an orientation of every $\Sigma_{i}$, and consider the induced orientation on every $C \in \pi_{0}\left(\partial \Sigma_{i}\right)$. Equip every $\Sigma_{i}$ with a Riemannian metric so that every $C \in \pi_{0}\left(\partial \Sigma_{i}\right)$ has a compact collar neighborhood

\footnotetext{
${ }^{3} \mathrm{~A}$ function of the radius in polar coordinates.
} 
isometric to the flat cylinder $\mathbb{R} / \mathbb{Z} \times[0,1 / 2]$, and these collar neighborhoods are disjoint from each other. Fix a distinguished point $p_{i}$ in the complement of the union of these collar neighborhoods.

Now, consider edge-colored partially directed graphs, $(G, \beta, \mathcal{O})$, whose vertex degrees are uniformly bounded by $c$, with $\beta: E(G) \rightarrow\{1, \ldots, c\}$, and such that the property of Remark 11.4 is satisfied. They define a compact transitive saturated subspace $\mathcal{G}_{*,+}^{\prime}(c) \subset \mathcal{G}_{*,+}^{\prime}$. For $[G, \beta, \mathcal{O}, v] \in \mathcal{G}_{*,+}^{\prime}(c)$ and any connected subgraph

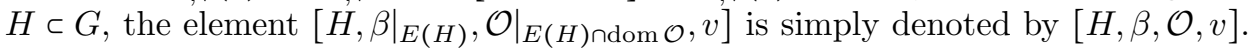
Consider the finite subset

$$
\mathbf{A}=\left\{[G, \beta, \mathcal{O}, v] \in \mathcal{G}_{*,+}^{\prime}(c) \mid \bar{B}_{G}(v, 1)=G\right\} \subset \mathcal{G}_{*,+}^{\prime}(c) .
$$

For any $\mathbf{a}=[G, \beta, \mathcal{O}, v] \in \mathbf{A}$, let $i_{\mathbf{a}}=\operatorname{deg}_{G}(v)$, which depends only on a. There is a continuous surjective map $\pi: \mathcal{G}_{*,+}^{\prime}(c) \rightarrow \mathbf{A}$ defined by $\pi([G, \beta, \mathcal{O}, v])=\left[\bar{B}_{G}(v, 1), \beta, \mathcal{O}, v\right]$, whose fibers, $\mathcal{Z}_{\mathbf{a}}=\pi^{-1}(\mathbf{a})(\mathbf{a} \in \mathbf{A})$, form a finite partition of $\mathcal{G}_{*,+}^{\prime}(c)$ by clopen subsets.

For every $\mathbf{a} \in \mathbf{A}$, let $\Sigma_{\mathbf{a}}$ be an isometric copy of $\Sigma_{i_{\mathbf{a}}}$ with the corresponding orientation, and let $p_{\mathbf{a}} \in \Sigma_{\mathbf{a}}$ be the point that corresponds to $p_{i_{\mathbf{a}}}$. For any edge $e$ of $G$ with vertex $v$, let

$$
\tau_{G, \mathcal{O}, v}(e)= \begin{cases}0 & \text { if } e \notin \operatorname{dom} \mathcal{O} \\ 1 & \text { if } e \in \operatorname{dom} \mathcal{O} \text { and } v \text { is the first vertex in } \mathcal{O}(e) \\ -1 & \text { if } e \in \operatorname{dom} \mathcal{O} \text { and } v \text { is the second vertex in } \mathcal{O}(e) .\end{cases}
$$

This defines a map $\tau_{G, \mathcal{O}, v}: E\left(\bar{B}_{G}(v, 1)\right) \rightarrow\{0, \pm 1\}$. The map

$$
\left(\beta, \tau_{G, \mathcal{O}, v}\right): E\left(\bar{B}_{G}(v, 1)\right) \rightarrow\{1, \ldots, c\} \times\{0, \pm 1\}
$$

is injective by the property of Remark 11.4, and therefore its image, $Q_{\mathbf{a}}$, has $i_{\mathbf{a}}$ elements. Then, for every $(k, \varepsilon) \in Q_{\mathbf{a}}$, let $C_{\mathbf{a}}^{k, \varepsilon}$ be the connected component of $\partial \Sigma_{\mathbf{a}}$ that corresponds to the connected component $C$ of $\partial \Sigma_{i_{\mathrm{a}}}$ with $\sigma_{Q_{\mathrm{a}}}(C)=(k, \varepsilon)$.

For $\mathbf{a}, \mathbf{b} \in \mathbf{A}$, if $\mathbf{z} \in \mathcal{Z}_{\mathbf{a}}$ is contiguous in $\mathcal{G}_{*,+}^{\prime}$ to $\mathbf{z}^{\prime} \in \mathcal{Z}_{\mathbf{b}}$, then there is a unique $(k, \varepsilon) \in Q_{\mathbf{a}}$ with $(k,-\varepsilon) \in Q_{\mathbf{b}}$. For $(k, \varepsilon) \in Q_{\mathbf{a}}$ with $(k,-\varepsilon) \in Q_{\mathbf{b}}$, fix an orientation reversing isometry $h_{\mathbf{a}, \mathbf{b}}^{k, \varepsilon}: C_{\mathbf{a}}^{k, \varepsilon} \rightarrow C_{\mathbf{b}}^{k,-\varepsilon}$ so that $h_{\mathbf{b}, \mathbf{a}}^{k,-\varepsilon}=\left(h_{\mathbf{a}, \mathbf{b}}^{k, \varepsilon}\right)^{-1}$. Then let $\mathcal{M G}_{*,+}^{\prime}(c)$ be the quotient of $\bigsqcup_{\mathbf{a}} \Sigma_{\mathbf{a}} \times \mathcal{Z}_{\mathbf{a}}$ by gluing every $(x, \mathbf{z}) \in C_{\mathbf{a}}^{k, \varepsilon} \times \mathcal{Z}_{\mathbf{a}}$ with $\left(h_{\mathbf{a}, \mathbf{b}}^{k, \varepsilon}(x), \mathbf{z}^{\prime}\right) \epsilon$ $C_{\mathbf{b}}^{k,-\varepsilon} \times \mathcal{Z}_{\mathbf{b}}$ if $\mathbf{z}$ and $\mathbf{z}^{\prime}$ are contiguous, $(k, \varepsilon) \in Q_{\mathbf{a}}$ and $(k,-\varepsilon) \in Q_{\mathbf{b}}$. The trivial Riemannian foliated structure on $\bigsqcup_{\mathbf{a}} \Sigma_{\mathbf{a}} \times \mathcal{Z}_{\mathbf{a}}$, defined by the fibers $\Sigma_{\mathbf{a}} \times\{\mathbf{z}\}\left(\mathbf{z} \in \mathcal{Z}_{\mathbf{a}}\right)$, can be projected to $\mathcal{M G}_{*,+}^{\prime}(c)$, which becomes a compact oriented Riemannian foliated space. There is an embedding $\mathcal{G}_{*,+}^{\prime}(c) \rightarrow \mathcal{M G}_{*,+}^{\prime}(c)$, assigning to every $\mathbf{z} \in \mathcal{G}_{*,+}^{\prime}(c)$ the projection to $\mathcal{M G}_{*,+}^{\prime}(c)$ of $\left(\mathbf{z}, p_{\mathbf{a}}\right) \in \Sigma_{\mathbf{a}} \times \mathcal{Z}_{\mathbf{a}}$, where $\mathbf{a}=\pi(\mathbf{z})$. This embedding realizes $\mathcal{G}_{*,+}^{\prime}(c)$ as a complete transversal of $\mathcal{M G}_{*,+}^{\prime}(c)$, and the canonical partition of $\mathcal{G}_{*,+}^{\prime}(c)$ is given by the orbits of the holonomy pseudogroup. Thus $\mathcal{M G}_{*,+}^{\prime}(c)$ is indeed a Riemannian matchbox manifold. Moreover every orbit closure $Z=\mathrm{Cl}_{+}^{\prime}\left(\mathrm{im} \iota_{G, \beta, \mathcal{O}}^{\prime}\right) \subset \mathcal{G}_{*,+}^{\prime}(c)$ determines a compact transitive saturated subspace $X=\mathcal{M} Z \subset \mathcal{M S}_{*,+}^{\prime}(c)$, called a graph matchbox manifold, which is minimal just when $(G, \beta, \mathcal{O})$ is repetitive, and its leaves are without holonomy just when $(G, \beta, \mathcal{O})$ is limit-aperiodic.

This kind of construction applies as well to the other graph spaces $\mathcal{T}=\mathcal{T}(\Gamma)$, $\widehat{\mathcal{T}}(c)=\widehat{\mathcal{T}}(\Gamma, c)$ and $\{0,1\}^{\Gamma}$ of Section 11.6 . defined by a finitely generated group $\Gamma$ and a finite set $S$ of generators such that $S \cap S^{-1}$ consists of elements of order 
two. We have to use their canonical partitions into graphs, equipped with edge colorings and partial directions satisfying the property of Remark 11.4 Then we get compact transitive Riemannian oriented matchbox manifolds, $\mathcal{M T}=\mathcal{M T}(\Gamma)$, $\mathcal{M} \widehat{\mathcal{T}}(c)=\mathcal{M} \widehat{\mathcal{T}}(\Gamma, c)$ and $\mathcal{M}\{0,1\}^{\Gamma}$, satisfying similar properties with respect to $\mathcal{T}, \widehat{\mathcal{T}}(c)$ and $\{0,1\}^{\Gamma}$. As before, we use the notation $X=\mathcal{M} Z$ for the compact transitive saturated subspace of $\mathcal{M T}, \mathcal{M} \widehat{\mathcal{T}}(c)$ or $\mathcal{M}\{0,1\}^{\Gamma}$ that corresponds to any transitive compact subspace $Z$ of $\mathcal{T}, \widehat{\mathcal{T}}(c)$ or $\{0,1\}^{\Gamma}$. The term graph matchbox manifold is also used for $X$ in the case of $\mathcal{M T}$ and $\mathcal{M T}(c)$, and the term subshift matchbox manifold is used for $X$ in the case of $\mathcal{M}\{0,1\}^{\Gamma}$.

Note that $\mathcal{M}\{0,1\}^{\Gamma}$ is diffeomorphic to the suspension of the left $\Gamma$-action on $\{0,1\}^{\Gamma}$ using a $\Gamma$-covering of the closed oriented surface of genus two. Similarly, $\mathcal{M T}$ can be considered as the "suspension" of its canonical compactly generated pseudogroup $\mathcal{H}$.

\subsection{Concrete examples of graph matchbox manifolds}

We give some concrete examples of graph matchbox manifolds, which easily illustrate the theorems stated in Chapter 1 .

11.9.1. The Ghys-Kenyon matchbox manifold. The Ghys-Kenyon tree 51 is the tree $T_{\infty} \in \mathcal{T}\left(\mathbb{Z}^{2}\right)$ defined as the limit of a sequence in $\mathcal{T}\left(\mathbb{Z}^{2}\right)$ whose first three terms are described in Figure 1, where the thick vertex represents the identity element $0 \in \mathbb{Z}^{2}$ (the distinguished vertex). These steps indicate the general procedure to construct the whole of $T_{\infty}$ by induction. The closure $Z$ of its class in $\mathcal{T}\left(\mathbb{Z}^{2}\right)$ is the Ghys-Kenyon graph space, and $X=\mathcal{M} Z \subset \mathcal{M T}\left(\mathbb{Z}^{2}\right)$ is called the Ghys-Kenyon matchbox manifold. This example is relevant because, first, it shows that parabolic and hyperbolic Riemann surfaces can be leaves of the same minimal compact foliated space, and, second, it was the first example constructed with this useful method.

The tree $T_{\infty}$ is $\mathbb{Z}^{2}$-limit-aperiodic and $\mathbb{Z}^{2}$-repetitive, and therefore $X$ is minimal and its leaves have no holonomy. The graph $T_{\infty}$ has four ends, and the corresponding leaf in $X$ also has four ends. Hence, by Corollary 1.4, every leaf of $X$ is coarsely quasi-isometric to meagerly many leaves (the alternative (ii) of Theorem 1.2); in particular, there are uncountably many coarse quasi-isometry types. This also follows using Theorem 1.3 , since $T_{\infty}$ is not coarsely quasi-symmetric; indeed, if $v_{n}$ is an unbounded sequence of vertices in $T_{\infty}$, and $\phi_{n}$ is a pointed coarse quasi-isometry of $\left(T_{\infty}, 0\right)$ to $\left(T_{\infty}, v_{n}\right)$ with coarse distortion $\left(K_{n}, C_{n}\right)$, then it is easy to check that the sequence $\left(K_{n}, C_{n}\right)$ is unbounded.

A coding introduced by Alcalde, Lozano and Macho establishes a Borelian open bijection [1, Proposition 3.3.2 and 3.4.1]

$$
\Phi:\{0,1,2,3\}^{\mathbb{N}} \equiv \mathbb{Z}_{4}^{\mathbb{N}} \rightarrow\left\{T \in Z \backslash\left\{T_{\infty}\right\} \mid \operatorname{deg}_{T}(0) \leq 2\right\} ;
$$

for instance, Figures 2 and 3 describe the processes to construct trees whose codings begin with 321 and 020 , which clarifies the general procedure. Note that the saturation of $\operatorname{im} \Phi$ is $Z \backslash\left\{T_{\infty}\right\}$. The classes of $\Phi(020202 \ldots)$ and $\Phi(131313 \ldots)$ are graphs with two ends, and all other classes in $Z \backslash\left\{T_{\infty}\right\}$ are graphs with one end. For $\alpha, \beta \in \mathbb{Z}_{4}^{\mathbb{N}}$, the classes of $\Phi(\alpha)$ and $\Phi(\beta)$ are equal if and only if $\alpha$ and $\beta$ are eventually equal [1, Proposition 3.2.1]. On the other hand, it is easy to check that the classes of $\Phi(\alpha)$ and $\Phi(\beta)$ are coarsely quasi-isometric if and only if $\alpha$ and $\beta$ are eventually equal up to permutations of $\mathbb{Z}_{4}$ defined by orthogonal transformations 

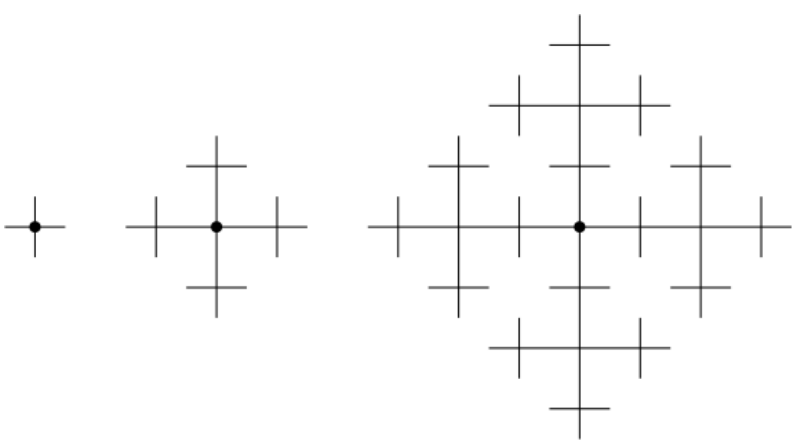

Figure 1. Ghys-Kanyon tree $T_{\infty}$

of $\mathbb{R}^{2}$ that preserve the set $\{(1,0),(0,1),(-1,0),(0,-1)\} \equiv \mathbb{Z}_{4}$, where this identity is given by $(1,0) \mapsto 0,(0,1) \mapsto 1,(-1,0) \mapsto 2$ and $(0,-1) \mapsto 3$. This confirms that $X$ has uncountable many coarse-quasi-isometry types.

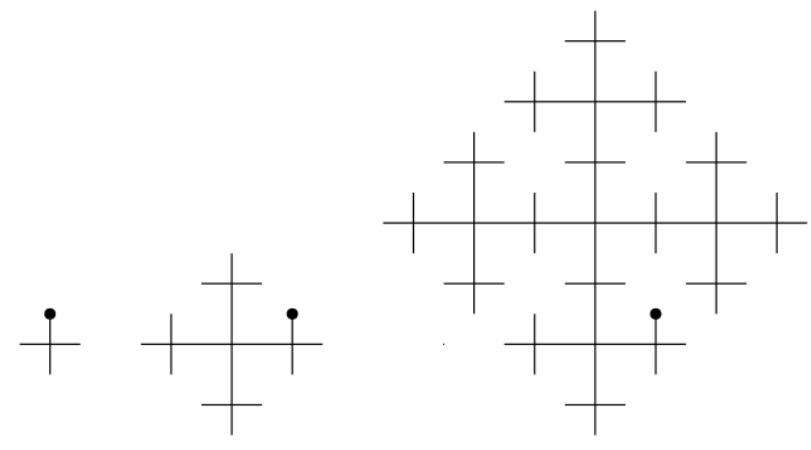

Figure 2. Tree with coding $321 \ldots$
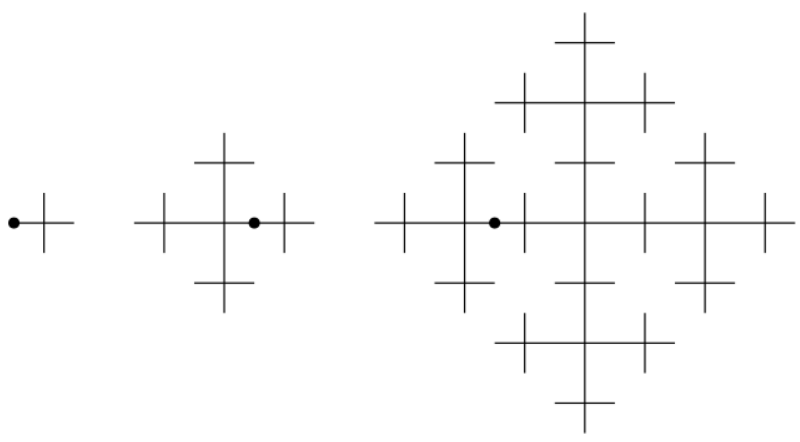

FiguRE 3. Tree with coding $020 \ldots$

The growth of the leaves of $X$ is equi-equivalent and quadratic, which can be checked as follows. For all $T \in Z$, since the distances of $\mathbb{Z}^{2}$ and $T$ satisfy the relation $d_{\mathbb{Z}^{2}} \leq d_{T}$ on $T$, we get $\left|\bar{B}_{T}(v, R)\right| \leq\left|\bar{B}_{\mathbb{Z}^{2}}(v, R)\right|$ for all $R>0$ and $v \in T$. If $T$ is not 
canonically equivalent to $\Phi(a a a \ldots)$ for any $a \in \mathbb{Z}_{4}$, then $T$ is a 1 -net in $\mathbb{Z}^{2}$, and therefore there is some $C>0$ such that $\left|B_{\mathbb{Z}^{2}}(v, R)\right| \leq C\left|T \cap \bar{B}_{\mathbb{Z}^{2}}(v, R)\right|$. However the restriction of $d_{\mathbb{Z}^{2}}$ to $T$ is not Lipchitz equivalent to $d_{T}$. But we have $d_{\mathbb{Z}^{2}}=d_{T}$ on at least half of the points of $\bar{B}_{\mathbb{Z}^{2}}(v, R)$. Thus

$$
\left|\bar{B}_{T}(v, R)\right| \geq \frac{1}{2}\left|T \cap \bar{B}_{\mathbb{Z}^{2}}(v, R)\right| \geq \frac{1}{2 C}\left|B_{\mathbb{Z}^{2}}(v, R)\right| .
$$

On the other hand, all trees $\Phi(a a a \ldots)\left(a \in \mathbb{Z}_{4}\right)$ are isometric to each other, as well as the trees in their classes. Thus, to check the remaining cases, we can assume $T=\Phi(000 \ldots)$. In this case, $T$ is a 1 -net in the quadrant

$$
Q=\left\{\left(v_{1}, v_{2}\right) \in \mathbb{Z}^{2}|| v_{2} \mid \leq v_{1}\right\}
$$

of $\mathbb{Z}^{2}$. Then we can repeat the above argument using $Q$ instead of $\mathbb{Z}^{2}$.

Many variants of the Ghys-Kennyon example were given by other authors 18, 74, 75, 78. The coarse quasi-isometry type of the leaves can be analyzed in all of them like in the Ghys-Kennyon example.

11.9.2. An example with uncountably many growth types of leaves. Let $F_{3}$ be the free group with three generators. We are going to construct a repetitive limit-aperiodic tree $T_{\infty} \in \mathcal{T}\left(F_{3}\right)$ that is not growth symmetric. Once $T_{\infty}$ is constructed, let $Z$ denote the closure of the class of $T_{\infty}$ in $\mathcal{T}\left(F_{3}\right)$, and let $X=\mathcal{M} Z \subset \mathcal{M T}\left(F_{3}\right)$. Then $X$ is minimal, its leaves have no holonomy, and the leaf that corresponds to $T_{\infty}$ is not growth symmetric. By Theorem 1.7, it follows that the growth type of each leaf of $X$ is comparable with the growth type of meagerly many leaves (the alternative (ii) of Theorem 1.6); in particular, $X$ has uncountably many growth types of leaves.

For the construction of $T_{\infty}$, we adapt the method of $\left[\mathbf{1 8}^{4}\right.$ Fix generators $a, b$ and $c$ of $F_{3}$. We identify the subgraphs of $F_{3}$ with their vertex set. Take strictly increasing sequences $r_{n}$ in $\mathbb{Z}^{+}$, and let $s_{n}=r_{1} \cdots r_{n}$ and $D_{n}=\bar{B}_{\langle b, c\rangle}(1, n)$. Consider the trees $T_{0}=\left\{a, a^{2}, \ldots\right\}$ and

$$
T_{1}=T_{0} \cup \bigcup_{n \geq 1} D_{1} a^{n s_{1}}
$$

For any segment $I \subset T_{0}$ and any tree $T$ containing $T_{0}$, the tree $T \cap F_{3}\left\{b^{ \pm 1}, c^{ \pm 1}\right\} I$ is called the $\langle b, c\rangle$-saturation of $I$ in $T$.

Beginning with the above trees $T_{0} \subset T_{1}$ and proceeding by induction, we continue constructing an increasing sequence, $T_{0} \subset T_{1} \subset \ldots$, in $\mathcal{T}\left(F_{3}\right)$. For some $n \geq 1$, assume that we have constructed $T_{0} \subset T_{1} \subset \cdots \subset T_{n}$ such that:

- they depend only on the terms $r_{1}, \ldots, r_{n}$ of the above sequence;

- for all $i=0,1, \ldots, n-1$, the segment $I_{i}:=\left\{a, \ldots, a^{s_{i}}\right\}$ has the same $\langle b, c\rangle$ saturation in $T_{i}$ and in $T_{i+1}$, which is denoted by $S_{i}$;

- for all $i=0,1, \ldots, n$ and $k \in \mathbb{Z}^{+}$, the $\langle b, c\rangle$-saturation of the segment $I_{i} a^{(k-1) s_{i}}$ in $T_{i}$ is $S_{i} a^{k s_{i}} ;$ and,

- if $r_{1}, \ldots, r_{n}$ are large enough, we can get

$$
\left|\bar{B}_{T_{i}}(a, r)\right| \leq \sum_{j=0}^{i} 2^{-j} r
$$

for all $i=0,1, \ldots, n$ and $r>0$.

\footnotetext{
${ }^{4} \mathrm{Be}$ aware that thet Caley graph structures of $[\mathbf{1 8}$ are left invariant, and ours are right invariant.
} 
These properties are obviously satisfied for $n=1$. Then

$$
T_{n+1}=T_{n} \cup \bigcup_{k=1}^{\infty} S_{n-1} D_{n+1} a^{k s_{n+1}}
$$

satisfies the desired properties; in particular, taking $r_{n+1}$ large enough, we can assume that (11.3) also holds for $i=n+1$ and all $r>0$.

Now, it is easy to check that $T_{\infty}=\bigcup_{n=0}^{\infty} T_{n}$ is an $F_{3}$-repetitive $F_{3}$-limit-aperiodic tree in $\mathcal{T}\left(F_{3}\right)$, which satisfies

$$
\left|\bar{B}_{T_{\infty}}(a, r)\right| \leq 2 r
$$

for all $r>0$ by $(11.3)$. But, for any $n \in \mathbb{Z}^{+}$, the ball $\bar{B}_{T_{\infty}}\left(a^{s_{n}}, n\right)$ contains $D_{n} a^{s_{n}}$, which is isometric to $D_{n}$ using the right translation by $a^{s_{n}}$. Thus

$$
\left|\bar{B}_{T_{\infty}}\left(a^{s_{n}}, r\right)\right| \geq\left|\bar{B}_{\langle b, c\rangle}(1, r)\right|=1+2\left(3^{r}-1\right) \geq 3^{r}
$$

for $0 \leq r \leq R_{n}$. Since $R_{n} \uparrow \infty$, comparing (11.4) and 11.5) it follows that $T_{\infty}$ cannot be growth symmetric.

11.9.3. An example with equi-amenable dense leaves and other nonamenable leaves. In Sections 11.9.1 and 11.9.2, it is easy to see that $T_{\infty}$ is amenable, and indeed all trees in $Z$ are jointly amenably symmetric. Hence all leaves of $X$ are jointly amenably symmetric.

Now, with the notation of Section 11.9.2, construct an increasing sequence, $T_{0}^{\prime} \subset T_{1}^{\prime} \subset \ldots$, in $\mathcal{T}\left(F_{3}\right)$ by induction, setting $T_{0}^{\prime}=T_{0}$ and

$$
T_{n+1}^{\prime}=T_{n}^{\prime} \cup \bigcup_{k=1}^{\infty} D_{n+1} a^{k s_{n+1}} .
$$

Let $T_{\infty}^{\prime}=\cup_{n} T_{n}^{\prime} \in \mathcal{T}\left(F_{3}\right)$, let $Z^{\prime}$ denote the closure of its class in $\mathcal{T}\left(F_{3}\right)$, and $X^{\prime}=\mathcal{M} Z^{\prime} \subset \mathcal{M T}\left(F_{3}\right)$. The tree $T_{\infty}^{\prime}$ is $F_{3}$-non-periodic, but it is neither $F_{3^{-}}$ limit-aperiodic nor $F_{3}$-repetitive; for instance, $Z^{\prime}$ contains the non-dense class that consists only of the tree $\langle b, c\rangle$. This class corresponds to a compact leaf with holonomy of $X^{\prime}$.

By Theorem 11.3 , there exists some limit-aperiodic coloring $\alpha$ of $T_{\infty}^{\prime}$ by 6 colors, which is also $F_{3}$-limit-aperiodic. But $\left(T_{\infty}, \alpha\right)$ is not $F_{3}$-repetitive because $T_{\infty}$ is not $F_{3}$-repetitive. Let $\widehat{Z}^{\prime}$ be the closure of the class of $\left(T_{\infty}, \alpha\right)$ in $\widehat{\mathcal{T}}\left(F_{3}, 6\right)$, and let $\widehat{X}^{\prime}=\mathcal{M} \widehat{Z}^{\prime} \subset \mathcal{M} \widehat{\mathcal{T}}\left(F_{3}\right)$. Now, the leaves of $\widehat{X}^{\prime}$ have no holonomy, and all dense leaves of $\widehat{X}^{\prime}$ are equi-amenable by Theorem 1.10 -(ii). But there are colored trees in $\widehat{Z}^{\prime}$ of the form $\left(\langle b, c\rangle, \alpha^{\prime}\right)$ for some $F_{3}$-limit-aperiodic colorings $\alpha^{\prime}$, whose classes are not dense in $\widehat{Z}^{\prime}$. Since $F_{2}$ is not Følner, the corresponding leaves are not amenable. This does not contradict Theorem 1.10 (ii) because these leaves are not dense.

11.9.4. An example with with leaves of infinite asymptotic dimension. With the notation of Section 11.8 , in $\mathcal{M T}(\Gamma)$ and $\mathcal{M} \widehat{\mathcal{T}}(\Gamma, c)$, all unbounded leaves have asymptotic dimension one, and all bounded leaves have asymptotic dimension zero. This holds because the asymptotic dimension of trees is one in the unbounded case, and zero in the bounded case [16.

To produce an example of graph matchbox manifold with leaves of infinite asymptotic dimension, take a finitely generated group $\Gamma$ with infinite asymptotic dimension, like the reduced wreath product of $\mathbb{Z}$ by $\mathbb{Z}[\mathbf{1 6}$. Section 5]. Then, in the matchbox manifold $\mathcal{M}\{0,1\}^{\Gamma}$, the leaves corresponding to non-periodic colorings are coarsely quasi-isometric to $\Gamma$, of infinite asymptotic dimension, and the leaves 
corresponding to constant colors are of zero asymptotic dimension because they are bounded.

Take a $\Gamma$-repetitive $\Gamma$-limit-aperiodic coloring $\alpha \in\{0,1\}^{\Gamma}$ (Section 11.6). Then the subshift matchbox manifold $X=\mathcal{M} \overline{\Gamma \cdot \alpha} \subset \mathcal{M}\{0,1\}^{\Gamma}$ is minimal and without holonomy. Moreover the leaves of $X$ are coarsely quasi-isometric to $\Gamma$, of infinite asymptotic dimension.

\subsection{Foliations of codimension one}

Now, we consider only transversely oriented $C^{2}$ foliations of codimension one on closed manifolds, unless otherwise stated. Then, unlike Theorem 11.1, there are examples of connected Riemannian manifolds of bounded geometry whose quasiisometry type cannot be realized as leaves $[\mathbf{1 2}, \mathbf{1 0 0}, \mathbf{9 0}$, 91. If the metric is not considered, any surface can be realized as a leaf of a codimension one foliation on a closed manifold [27, but this fails in higher dimension [49, 69, [12, 94, 92].

11.10.1. Hector's example. Concerning the growth of the leaves, let us recall the construction of a striking example of a transitive $C^{\infty}$ foliation given by Hector [62, whose properties were indicated in Chapter 1. This example was originally described in his previous paper [60, which is a great source of examples that can be similarly analyzed in terms of our main theorems.

By using the suspension construction (Section 11.1), it is enough to describe a finitely generated group of orientation preserving $C^{\infty}$ diffeomorphisms of $S^{1}$; indeed, this is achieved by describing a finitely generated group $G$ of orientation preserving $C^{\infty}$ diffeomorphisms of $[-1,1]$ that are flat ${ }^{5}$ at \pm 1 . Let $E([-1,1])$ be the set of orientation preserving $C^{\infty}$ diffeomorphisms $f$ of $[-1,1]$ such that $f$ is flat at \pm 1 , and the support ${ }^{6}$ of $f$ is an interval of the form $[\bar{a}, a]$, with $\bar{a}<0<a$, so that $f^{\prime}>1$ on $(\bar{a}, 0)$, and $f^{\prime}<1$ on $(0, a)$. There exists a sequence $k_{n}(n \in \mathbb{N})$ in $E([-1,1])$, with corresponding supports $\left[\bar{a}_{n}, a_{n}\right]$, such that:

- $\left[\bar{a}_{0}, a_{0}\right]=[-1,1], a_{n+1}=k_{n}\left(\bar{a}_{n+1}\right)$ and $\cap_{n}\left[\bar{a}_{n}, a_{n}\right]=\{0\} ;$ and,

- if $h=k_{0}, k_{(n)}=h^{-n} k_{n} h^{n}$ and $l_{n}=k_{(n)} \cdots k_{(1)}(n \geq 1)$, then $l=\lim _{n} l_{n}$ is a orientation preserving $C^{\infty}$ diffeomorphism of $[-1,1]$ which is flat at \pm 1 .

Let $G$ is the group generated by $\Sigma=\left\{h^{ \pm 1}, l^{ \pm 1}\right\}$. Every trajectory $T_{n}=G \cdot a_{n}$ is proper, and, for all $x \in \Omega=[-1,1] \backslash \cup_{n} \overline{T_{n}}$, the trajectory $T_{x}=G \cdot x$ is dense.

Consider the length $|\cdot|$ and right invariant metric on $G$ defined by $\Sigma$, and consider the induced metric $\delta$ on every trajectory $T_{x} \equiv G / G_{x}$. A short cut from $x$ to $y$ in $T_{x}$ is an element $g \in G$ such that $y=g(x)$ and $|g|=\delta(x, y)$. The notation $\Gamma_{x}$ is used for the set of short cuts from $x$. The short cuts satisfy the following properties:

- For all $x \in[-1,1]$ and $y \in T_{x}$, there is a unique short cut $g_{x, y}$ from $x$ to $y$.

- Any non-trivial $g \in G$ is in $\Gamma_{n}=\Gamma_{a_{n}}$ if and only if there exists some $p \in \mathbb{Z}^{+}$ and $\left(\alpha_{1}, \ldots, \alpha_{p}\right) \in \mathbb{Z}^{p}$ such that:

$-g=h^{\alpha_{1}}$ if $p=1 ;$ and

- $g=h^{\alpha_{1}} l^{\alpha_{q}} h \cdots h l^{\alpha_{p}} h^{-(p-1)}$ with $1<q \leq p, \alpha_{q} \alpha_{p} \neq 0$, and $\alpha_{j}=0$ for $1<j<q$, if $p>1$.

- For all $u, v \in T_{n}$, we have $g_{u, v}=g_{v} g_{u}^{-1}$, where $g_{u}=g_{a_{n}, u}$.

\footnotetext{
${ }^{5}$ They have the same derivatives of any order as the identity map at those points.
}

${ }^{6}$ The closure of the set of points where $f \neq$ id. 
- For all $x \in \Omega$ and $y \in T_{x}$, there exist $n \in \mathbb{N}$ and $u, v \in T_{n}$ such that $g_{x, y}=g_{u, v}$.

Thus $\Gamma_{x} \equiv T_{x} \equiv G / G_{x}$ as graphs; in particular, $\operatorname{gr}\left(\Gamma_{x}\right)=\operatorname{gr}\left(T_{x}\right)=\operatorname{gr}\left(G / G_{x}\right)$. It also follows that, for each connected component $(\bar{u}, u)$ of $[-1,1] \backslash \overline{T_{n}}$, there is a unique $g=h^{\alpha_{1}} l^{\alpha_{q}} h \cdots h l^{\alpha_{p}} h^{-(p-1)} \in \Gamma_{n}$ such that $(\bar{u}, u)=g\left(\bar{a}_{n}, a_{n}\right)$. This procedure assigns an $n$-tuple $\left(\alpha_{1}, \ldots, \alpha_{n}\right)$ to such $(\bar{u}, u)$, taking $\alpha_{j}=0$ for $p<j \leq n$ if $p<n$. If $\left(\bar{u}^{\prime}, u^{\prime}\right) \subset(\bar{u}, u)$ is a connected component of $[-1,1] \backslash \overline{T_{n+1}}$, then the corresponding $(n+1)$-tuple $\left(\alpha_{1}^{\prime}, \ldots, \alpha_{n+1}^{\prime}\right)$ satisfies $\alpha_{j}^{\prime}=\alpha_{j}$ for $1 \leq j \leq n$. Since any point in $\Omega$ is the intersection of a decreasing sequence of intervals that are connected components of the complements $[-1,1] \backslash \overline{T_{n}}$, for all $n$, this procedure defines a bijection $\Phi: \Omega \rightarrow \mathbb{Z}^{\mathbb{Z}^{+}}$, which turns out to be a homeomorphism.

Considering $\Phi$ as an identity, for $x=\left(x_{n}\right) \in \Omega$ and $p \in \mathbb{Z}^{+}$, write $X_{p}=\sum_{n=1}^{p}\left|x_{n}\right|$. It is said that $x$ is weakly dominated by another point $y=\left(y_{n}\right) \in \Omega$ if there is some $A \in \mathbb{Z}^{+}$such that $X_{p} \leq A\left(p+Y_{p}\right)$ for all $p$. If $x$ and $y$ weakly dominate each other, then they are said to be weakly equivalent. Every weakly equivalence class has the cardinality of the continuum because it does not change by adding elements of $\{0,1\}^{\mathbb{Z}^{+}}$. The following properties hold:

- $T_{x}=T_{y}$ if and only if $\left(x_{n}\right)$ and $\left(y_{n}\right)$ are eventually equally.

- If $x$ is weakly dominated by $y$, then $\operatorname{gr}\left(\Gamma_{x}\right) \geq \operatorname{gr}\left(\Gamma_{y}\right)$.

The properties indicated in Chapter 1 follow by pursuing further this kind of concepts and arguments. More precisely, the following properties hold:

- Every growth class of metric spaces $\Gamma_{x}(x \in \Omega)$ has the cardinality of the continuum.

- Every $T_{n}$ has polynomial growth of exact degree $n$, and the growth of $T_{x}$ is non-polynomial for all $x \in \Omega$.

- $\Gamma_{0}$ has exponential growth.

- For $r>0$, let $x(r)=\left(x_{n}(r)\right) \in \Omega$ with $x_{n}(r)=\left\lfloor n^{r}\right\rfloor$. Then $\Gamma_{r}=\Gamma_{x(r)}$ has non-polynomial and quasi-polynomial growth. Moreover $\operatorname{gr}\left(\Gamma_{r}\right)<\operatorname{gr}\left(\Gamma_{s}\right)$ if $r>s$, and therefore there is a continuum of growth classes of this kind.

- For $r>0$, let $\tilde{x}(r)=\left(\tilde{x}_{n}(r)\right) \in \Omega$ with

$$
\tilde{x}_{n}(r)= \begin{cases}X_{p}(r)-X_{p-1}(r) & \text { if } n=X_{p}(r)=\sum_{n=1}^{p}\left\lfloor n^{r}\right\rfloor \\ 0 & \text { if } n \notin\left\{X_{p}(r) \mid p \in \mathbb{Z}^{+}\right\} .\end{cases}
$$

Then $\widetilde{\Gamma}_{r}=\Gamma_{\tilde{x}(r)}$ has non-quasi-polynomial and non-exponential growth. Moreover $\operatorname{gr}\left(\widetilde{\Gamma}_{r}\right)<\operatorname{gr}\left(\widetilde{\Gamma}_{s}\right)$ if $r>s$, and therefore there is a continuum of growth classes of this kind.

We will show that the growth type of every leaf is comparable with the growth type of meagerly many leaves (the alternative (ii) of Theorem 1.6); in particular, there are uncountably many growth types of leaves without holonomy. Actually, a stronger property will be proved in Section 11.10.4, whose statement and proof requires concepts and results recalled in Sections 11.10.2 and 11.10.3.

11.10.2. Generic ergodicity of equivalence relations. The following concepts are used. The orbits of an action of a group $G$ on a space $X$ define an equivalence relation denoted by $E_{G}^{X}$. A metric with possible infinite values ${ }^{7}$ on a space $X$, $d: X \times X \rightarrow[0, \infty]$, defines an equivalence relation $E_{d}^{X}=d^{-1}([0, \infty))$ on $X$, called a

\footnotetext{
${ }^{7}$ It is defined like a usual metric, except that the infinite distance between points is possible.
} 
metric equivalence relation. Any metric with possible infinite values induces a topology like usual metrics. The composite of relations is the obvious extension of the composite of maps. For $E \subset X^{2}, x \in X$ and $S \subset X$, let $E(x)=\{y \in X \mid(x, y) \in E\}$ and $E(S)=\cup_{z \in X} E(z)$. The identity relation at $X$ is the diagonal $\Delta_{X} \subset X^{2}$.

Let $E$ and $F$ be equivalence relations on respective spaces $X$ and $Y$. A map $\theta: X \rightarrow Y$ is called $(E, F)$-invariant if $\left(\theta(x), \theta\left(x^{\prime}\right)\right) \in F$ for all $\left(x, x^{\prime}\right) \in E$; this means that $\theta$ induces a map $\bar{\theta}: X / E \rightarrow Y / F$. It is said that $E$ is Borel reducible to $F$, written $E \leq_{B} F$, if there is an $(E, F)$-invariant Borel map $\theta: X \rightarrow Y$ such that, for all $x, x^{\prime} \in X$, we have $\left(x, x^{\prime}\right) \in E$ if $\left(\theta(x), \theta\left(x^{\prime}\right)\right) \in F$; this means that $\bar{\theta}: X / E \rightarrow Y / F$ is injective. If $E \leq_{B} F \leq_{B} E$, then $E$ is said to be Borel bi-reducible with $F$, and the notation $E \sim_{B} F$ is used. On the contrary, it is said that $E$ is generically $F$-ergodic if, for any $(E, F)$-invariant Baire measurable map $\theta: X \rightarrow Y$, there is some residual saturated $C \subset X$ such that $\bar{\theta}: C /\left(E \cap C^{2}\right) \rightarrow Y / F$ is constant.

The partial pre-order relation $\leq_{B}$ establishes a hierarchy on the complexity of equivalence relations on Polish spaces. A first rank of this hierarchy consists of the concretely classifiable or smooth equivalence relations, defined by the condition of being $\leq_{B} \Delta_{\mathbb{R}}$; this means that their equivalence classes can be distinguished by some Borel map to $\mathbb{R}$.

Consider the Polish space $\prod_{n=1}^{\infty}\{0,1\}^{\mathbb{N}^{n}}$, with the canonical action of the Polish group $S_{\infty}$ of permutations of $\mathbb{N}$. Each element of $\prod_{n=1}^{\infty}\{0,1\}^{\mathbb{N}^{n}}$ can be considered as a structure on $\mathbb{N}$ defined by a sequence of relations $R_{n}$ with arity $n$ (subsets of $\mathbb{N}^{n}$ ); the term countable model is used for $\mathbb{N}$ with this structure. The $S_{\infty}$-action defines the isomorphism relation $\cong$ between countable models. A second classification rank consists of equivalence relations $\leq_{B} \cong$, which are said to be classifiable by countable models . On the contrary, we have the generically $\cong$-ergodic relations; indeed, these relations are generically $E_{S_{\infty}}^{Y}$-ergodic for any Polish $S_{\infty}$-space $Y$ [66] (see also [15, 72]).

Consider the equivalence relation $E_{G}^{X}$ defined by a Polish action 8 . For open neighborhoods, $U$ of a point $x$ in $X$ and $V$ of the identity element in $G$, let $\mathcal{O}(x, U, V)$ denote the set of points $y \in U$ such that there is a finite sequence $x=x_{0}, x_{1}, \ldots, x_{n}=y$ in $U$, for some $n \in \mathbb{Z}^{+}$, so that $x_{i} \in V \cdot x_{i-1}$ for all $i=1, \ldots, n$. This set $\mathcal{O}(x, U, V)$ is called a local orbit. This action is said to be turbulent if its orbits are dense and meager, and its local orbits are somewhere dense $9^{9}$. Hjorth has introduced this dynamical property, and used it to give a precise characterization

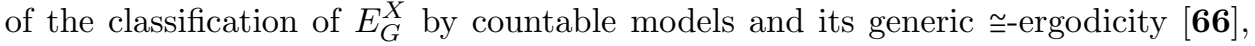
67 .

11.10.3. Generic ergodicity of metric equivalence relations. We recall our partial extension of Hjorth's work to metric equivalence relations $\mathbf{9}$.

On a Polish space $X$, consider the metric equivalence relation $E_{d}^{X}$ defined by a metric $d$ with possible infinite values. For every open neighborhood $U$ of any point $x$ in $X$, and all $\varepsilon>0$, let $E_{d}^{X}(x, U, \varepsilon)$ denote the set of points $y \in U$ such that there is a finite sequence $x=x_{0}, x_{1}, \ldots, x_{m}=y$ in $U$, for some $m \in \mathbb{Z}^{+}$, so that $d\left(x_{i-1}, x_{i}\right)<\varepsilon$ for all $i=1, \ldots, m$. This set $E_{d}^{X}(x, U, \varepsilon)$ is called a local equivalence class. It is said that $E_{d}^{X}$ is turbulent if its equivalence classes are dense and meager, and its local equivalence classes are somewhere dense. If $d$ is of certain class, called type $I$ in $\mathbf{9}$,

\footnotetext{
${ }^{8}$ The actionof a Polish group on a Polish space.

${ }^{9}$ The closure has nonempty interior.
} 
and $E_{d}^{X}$ is turbulent, then $E_{d}^{X}$ is generically $E_{S_{\infty}}^{Y}$-ergodic for any Polish $S_{\infty}$-space $Y$ [9, Theorem 5.5].

Now, let $X$ be a set, and let $\mathcal{U}=\left\{U_{R, r} \subset X^{2} \mid R, r>0\right\}$ be a set of relations on $X$. The following list of hypotheses are used to define a metric equivalence relation satisfying the above conditionss.

HYPOTHESIS 3. (i) $\cap_{R, r>0} U_{R, r}=\Delta_{X}$;

(ii) each $U_{R, r}$ is symmetric;

(iii) if $R \leq S$, then $U_{R, r} \supset U_{S, r}$ for all $r>0$;

(iv) $U_{R, r}=\bigcup_{s<r} U_{R, s}$ for all $R, r>0$; and

(v) there is some function $\phi:\left(\mathbb{R}^{+}\right)^{2} \rightarrow \mathbb{R}^{+}$such that, for all $R, S, r, s>0$,

$$
\begin{gathered}
R \leq \phi(R, r), \\
(R \leq S, r \leq s) \Longrightarrow \phi(R, r) \leq \phi(S, s), \\
U_{\phi(R, r+s), r} \circ U_{\phi(R, r+s), s} \subset U_{R, r+s .} .
\end{gathered}
$$

Under Hypothesis 3 , the sets $U_{R, r}$ form a base of entourages for a Hausdorff metrizable uniformity $\mathcal{U}$ on $X$, and, setting $E_{r}=\bigcap_{R>0} U_{R, r}(r>0)$, a metric with possible infinite values, $d: X \times X \rightarrow[0, \infty]$, is defined by

$$
d(x, y)=\inf \left\{r>0 \mid(x, y) \in E_{r}\right\} .
$$

Hypothesis $4 . \quad$ (i) $X$ is a Polish space with the topology induced by $\mathcal{U}$;

(ii) for all $R, r, s>0$ and $x \in X$, if $y \in E_{s}(x)$, then there are some $T, t>0$ such that $U_{T, t}(y) \subset E_{s} \circ U_{R, r}(x)$; and,

(iii) for all $r, s>0$ and $(x, y) \in E_{s}$, and any neighborhood $V$ of $y$ in $X$, there is some neighborhood $W$ of $y$ in $X$ such that

$$
E_{r}(W) \cap E_{r}\left(E_{s}(x)\right) \subset E_{r}\left(V \cap E_{s}(x)\right) .
$$

Under Hypothesis $4 d$ is of type I.

Hypothesis $5 . \quad$ (i) $E_{d}^{X}$ has more than one equivalence class;

(ii) for all $x, y \in X$ and $R, r>0$, there is $s>0$ such that $U_{R, r}(x) \cap E_{s}(y) \neq \varnothing$; and,

(iii) for each $x \in X$ and each $R, r>0$, there are $S, s>0$, a dense subset $\mathcal{D} \subseteq$ $U_{S, s}(x) \cap E_{d}^{X}(x)$, and a $d$-dense subset of $\mathcal{D}$ such that every pair of points in $\mathcal{D}$ can be joined by a $d$-continuous path in $U_{R, r}(x)$.

Under Hypothesis $5, E_{d}^{X}$ is turbulent. Thus, assuming Hypotheses 3 , the relation $E_{d}^{X}$ is generically $E_{S_{\infty}}^{Y}$-ergodic for any Polish $S_{\infty}$-space $Y$, 9, Proposition 6.10].

REMARK 11.5. Actually, a stronger version of Hypotheses 5.(iii) was required in [9. Hypothesis 3-(iii)]. That condition was simpler to state and check, and it was satisfied in the applications of that paper, and it was used in [9. Proposition 6.8] to obtain that the local equivalence classes are somewhere dense. But now we need the weaker requirement Hypotheses 5-(iii). Clearly, it also implies that the local equivalence classes are somewhere dense, and therefore this change can be made.

11.10.4. Generic ergodicity of the growth type relation in Hector's example. Consider the notation of Section 11.10.1.

TheOREM 11.6. In the Hector's foliation, the relation of being in leaves with the same growth type is generically $E_{S_{\infty}}^{Y}$-ergodic for any Polish $S_{\infty}$-space $Y$. 
Let $\Omega_{+}=\mathbb{N}^{\mathbb{Z}^{+}} \subset \Omega$. The weak equivalence relation on $\Omega$ is Borel bi-reducible to its restriction to $\Omega_{+}$. This can be seen by using the Borel maps $\Omega_{+} \hookrightarrow \Omega$ and $\Omega \rightarrow \Omega_{+},\left(x_{n}\right) \mapsto\left(\left|x_{n}\right|\right)$. In turn, the weak equivalence relation on $\Omega_{+}$has an obvious extension to the larger space $\widetilde{\Omega}_{+}=[0, \infty)^{\mathbb{Z}^{+}}$. The weak equivalence relations on $\Omega_{+}$ and $\widetilde{\Omega}_{+}$are Borel bi-reducible, as can be shown with the Borel maps $\Omega_{+} \hookrightarrow \widetilde{\Omega}_{+}$ and $\widetilde{\Omega}_{+} \rightarrow \Omega_{+},\left(x_{n}\right) \mapsto\left(\left\lfloor x_{n}\right\rfloor\right)$. So, according to Section 11.10.1. Theorem 11.6 is a consequence of the following proposition.

Proposition 11.7. The weak equivalence relation on $\widetilde{\Omega}_{+}$is generically $E_{S_{\infty}}^{Y}$ ergodic for any Polish $S_{\infty}$-space $Y$.

Using $\widetilde{\Omega}_{+}$has some advantages in the proof. First, the assignment $\left(x_{n}\right) \mapsto\left(X_{p}\right)$ defines a bijection between $\Omega_{+}$(respectively, $\widetilde{\Omega}_{+}$) and the set of non-decreasing sequences in $\mathbb{N}$ (respectively, $[0, \infty)$ ); second, it is easier to construct elements in $\widetilde{\Omega}_{+}$than in $\Omega_{+}$or $\Omega$; and, third, $\widetilde{\Omega}_{+}$has nontrivial continuous paths.

Proposition 11.7 follows by checking the hypotheses of Section 11.10 .3 are satisfied by the relations

$$
U_{R, r}=\left\{(x, y) \in \widetilde{\Omega}_{+}^{2} \mid e^{-r}\left(p+Y_{p}\right)<p+X_{p}<e^{r}\left(p+Y_{p}\right) \forall p=1, \ldots,\lfloor R\rfloor\right\},
$$

for $R, r>0$. These sets obviously satisfy Hypothesis 3 in particular, its condition (V) holds with $\phi(R, r)=R$ because

$$
U_{R, r} \circ U_{S, s} \subset U_{\min \{R, S\}, r+s} .
$$

Note that Hypothesis 3 (i) would not be true with the same definition of sets $U_{R, r}$ in $\Omega$. The uniformity defined by the sets $U_{R, r}$ induces the topology of $\widetilde{\Omega}_{+}$.

According to Section 11.10 .3 the sets $U_{R, r}$ induce the relations

$$
E_{r}=\left\{(x, y) \in \widetilde{\Omega}_{+}^{2} \mid e^{-r}\left(p+Y_{p}\right)<p+X_{p}<e^{r}\left(p+Y_{p}\right) \forall p \in \mathbb{Z}^{+}\right\},
$$

for $r>0$, which in turn induce the metric with possible infinite values, $d: \widetilde{\Omega}_{+}^{2} \rightarrow$ $[0, \infty]$, determined by

$$
e^{d(x, y)}=\inf \left\{A \in[1, \infty) \mid A^{-1}\left(p+Y_{p}\right)<p+X_{p}<A\left(p+Y_{p}\right) \forall p \in \mathbb{Z}^{+}\right\} .
$$

Note that $E_{d}^{\widetilde{\Omega}_{+}}=\bigcup_{r>0} E_{r}$ is the weak equivalence relation on $\widetilde{\Omega}_{+}$.

Lemma 11.8. $E_{r} \circ E_{s}=E_{r+s}$ for all $r, s>0$.

Proof. The inclusion "ᄃ" follows from (11.6). To prove "כ", take any $(x, y) \in$ $E_{r+s}$. An element $z \in E_{r}(x) \cap E_{s}(y)$ is determined by the condition

$$
p+Z_{p}=\left(p+X_{p}\right)^{\frac{s}{r+s}}\left(p+Y_{p}\right)^{\frac{r}{r+s}}
$$

for all $p \in \mathbb{Z}^{+}$. Therefore $(x, y) \in E_{r} \circ E_{s}$.

Hypothesis 4 (i) is true because $\widetilde{\Omega}_{+}$is Polish. The following lemma shows that Hypothesis 4 (ii) is also satisfied.

Lemma 11.9. For all $R, r, s>0, U_{R, r} \circ E_{s}=E_{s} \circ U_{R, r}=U_{R, r+s}$.

Proof. If $S \geq R$, then, by 11.6),

$$
\left(U_{R, r} \circ E_{s}\right) \cup\left(E_{s} \circ U_{R, r}\right) \subset\left(U_{R, r} \circ U_{S, s}\right) \cup\left(U_{S, s} \circ U_{R, r}\right) \subset U_{R, r+s} .
$$

Let us prove that

$$
U_{R, r+s} \subset\left(U_{R, r} \circ E_{s}\right) \cap\left(E_{s} \circ U_{R, r}\right) .
$$


For any $(x, y) \in U_{R, r+s}$, there is some $z \in U_{R, r}(x) \cap E_{s}(y)$, which can be defined by using (11.7) for $p \leq\lfloor R\rfloor$, and taking $z_{n}=y_{n}$ for $n>\lfloor R\rfloor$. Similarly, there is some $z^{\prime} \in U_{R, r}(y) \cap E_{s}(x)$. Thus $(x, y) \in\left(U_{R, r} \circ E_{s}\right) \cap\left(E_{s} \circ U_{R, r}\right)$.

Note that the equality in 11.6 follows from Lemma 11.9

Lemma 11.10. For all $T, r, s, t>0$ and $(x, y) \in E_{s}$, if $U_{T, t}(y) \subset U_{T, s}(x)$, then

$$
U_{T, t+r}(y) \cap E_{r+s}(x)=E_{r}\left(U_{T, t}(y) \cap E_{s}(x)\right) .
$$

Proof. The inclusion " $\supset$ " holds for all $t>0$ by Lemmas 11.8 and 11.9 .

To prove "כ", without lost of generality, we can assume that $T \in \mathbb{Z}^{+}$. Let $z \in$ $U_{T, t+r}(y) \cap E_{r+s}(x)$. By Lemmas 11.8 and 11.9 , there are elements $x^{\prime} \in E_{r}(z) \cap E_{s}(x)$ and $y^{\prime} \in E_{r}(z) \cap U_{T+1, t}(y)$. Take a sequence $0<\varepsilon_{p} \downarrow 0$ such that

$$
\begin{gathered}
e^{\varepsilon_{p}-s}\left(p+X_{p}\right), e^{\varepsilon_{p}-r}\left(p+Z_{p}\right)<p+X_{p}^{\prime}<e^{s-\varepsilon_{p}}\left(p+X_{p}\right), e^{r-\varepsilon_{p}}\left(p+Z_{p}\right), \\
e^{\varepsilon_{p}+\varepsilon_{p+1}}\left(p+X_{p}\right)<p+1+X_{p+1}, \\
\varepsilon_{p}+\varepsilon_{p+1}<r, t,
\end{gathered}
$$

for all $p$, and

$$
e^{\varepsilon_{p}-t}\left(p+Y_{p}\right), e^{\varepsilon_{p}-r}\left(p+Z_{p}\right)<p+Y_{p}^{\prime}<e^{t-\varepsilon_{p}}\left(p+Y_{p}\right), e^{r-\varepsilon_{p}}\left(p+Z_{p}\right),
$$

for $p \leq T+1$. Then other elements, $x^{\prime \prime} \in E_{r}(z) \cap E_{s}(x)$ and $y^{\prime \prime} \in E_{r}(z) \cap U_{T, t}(y)$, can be defined by

$$
\begin{aligned}
p+X_{p}^{\prime \prime} & =\min \left\{e^{s-\varepsilon_{p}}\left(p+X_{p}\right), e^{r-\varepsilon_{p}}\left(p+Z_{p}\right)\right\}, \\
p+Y_{p}^{\prime \prime} & = \begin{cases}\max \left\{e^{\varepsilon_{p}-t}\left(p+Y_{p}\right), e^{\varepsilon_{p}-r}\left(p+Z_{p}\right)\right\} & \text { if } p \leq T \\
p+Y_{p-1}^{\prime \prime}+z_{p} & \text { if } p>T .\end{cases}
\end{aligned}
$$

For $p \leq T$, if

$$
p+1+X_{p+1}^{\prime \prime}=e^{s-\varepsilon_{p+1}}\left(p+1+X_{p+1}\right), \quad p+Y_{p}^{\prime \prime}=e^{\varepsilon_{p}-p}\left(p+Y_{p}\right),
$$

then

If

$$
\frac{p+Y_{p}^{\prime \prime}}{p+1+X_{p+1}^{\prime \prime}}<\frac{e^{\varepsilon_{p}-t}\left(p+X_{p}\right)}{e^{-\varepsilon_{p+1}}\left(p+1+X_{p+1}\right)}<e^{-t}<1 .
$$

$$
p+1+X_{p+1}^{\prime \prime}=e^{r-\varepsilon_{p+1}}\left(p+1+Z_{p+1}\right), \quad p+Y_{p}^{\prime \prime}=e^{\varepsilon_{p}-r}\left(p+Z_{p}\right),
$$

then

If

$$
\frac{p+Y_{p}^{\prime \prime}}{p+1+X_{p+1}^{\prime \prime}}<e^{\varepsilon_{p}+\varepsilon_{p+1}-2 r}<1
$$

$$
p+1+X_{p+1}^{\prime \prime}=e^{s-\varepsilon_{p+1}}\left(p+1+X_{p+1}\right), \quad p+Y_{p}^{\prime \prime}=e^{\varepsilon_{p}-r}\left(p+Z_{p}\right),
$$

then

$$
\frac{p+Y_{p}^{\prime \prime}}{p+1+X_{p+1}^{\prime \prime}}<\frac{e^{\varepsilon_{p}}\left(p+X_{p}\right)}{e^{-\varepsilon_{p+1}}\left(p+1+X_{p+1}\right)}<1 .
$$

If

$$
p+1+X_{p+1}^{\prime \prime}=e^{r-\varepsilon_{p+1}}\left(p+1+Z_{p+1}\right), \quad p+Y_{p}^{\prime \prime}=e^{\varepsilon_{p}-t}\left(p+Y_{p}\right),
$$

then

$$
\frac{p+Y_{p}^{\prime \prime}}{p+1+X_{p+1}^{\prime \prime}}<\frac{e^{\varepsilon_{p}-t}\left(p+Z_{p}\right)}{e^{-\varepsilon_{p+1}}\left(p+1+Z_{p+1}\right)}<e^{\varepsilon_{p}+\varepsilon_{p+1}-t}<1 .
$$


In any case, we have $p+Y_{p}^{\prime \prime}<p+1+X_{p+1}^{\prime \prime}$. Therefore an element $z^{\prime} \in E_{r}(z) \cap$ $U_{T, t}(y) \cap E_{s}(x)$ can be defined by

$$
p+Z_{p}^{\prime}= \begin{cases}p+Y_{p}^{\prime \prime} & \text { if } p \leq T \\ p+X_{p}^{\prime \prime} & \text { if } p>T .\end{cases}
$$

Hence $z \in E_{r}\left(U_{T, t}(y) \cap E_{s}(x)\right)$.

Hypothesis 4-(iii) follows from Lemma 11.10 in the following way. Given $r, s>0$ and $(x, y) \in E_{s}$, and any neighborhood $V$ of $y$ in $\widetilde{\Omega}_{+}$. Since $V$ can be chosen as small as desired, we can assume that $V=U_{T, t}(y)$ for some $T, t>0$. From $y \in E_{s}(x) \subset U_{T, s}(x)$, we easily get some $t>0$ such that $U_{T, t}(y) \subset U_{T, s}(x)$. Then Lemmas 11.8 and 11.9 and 11.10 give the inclusion of Hypothesis 4 (iii) with $W=V$.

Hypothesis 5tii means that there are more than one weak equivalence class in $\widetilde{\Omega}_{+}$, which is indicated in Section 11.10.1. Hypothesis 5-(ii),(iii) are consequences of the following lemmas, completing the proof of Proposition 11.7.

LEMmA 11.11. For all $R, r, s>0$ and $(x, y) \in U_{R, s}$, we have $U_{R, r}(x) \cap E_{s}(y) \neq \varnothing$.

Proof. An element $z \in U_{R, r}(x) \cap E_{s}(y)$ can be defined by

$$
z_{n}= \begin{cases}x_{n} & \text { if } n \leq\lfloor R\rfloor \\ y_{n} & \text { if } n>\lfloor R\rfloor .\end{cases}
$$

LEMma 11.12. For every $R, r>0$ and every $x \in \widetilde{\Omega}_{+}$, the set $U_{R, r}(x) \cap E_{d}^{\widetilde{\Omega}_{+}}(x)$ is d-path connected.

Proof. For every $y \in U_{R, r}(x) \cap E_{d}^{\widetilde{\Omega}_{+}}(x)$, a $d$-continuous path $t \mapsto z(t)$ in $U_{R, r}(x) \cap E_{d}^{\widetilde{\Omega}_{+}}$from $y$ to $x$ can be defined by

$$
p+Z_{p}(t)=\left(p+X_{p}\right)^{t}\left(p+Y_{p}\right)^{1-t} .
$$

11.10.5. The theory of levels. For the reader's convenience, we briefly recall some concepts of this theory [25, which which are needed to understand the examples indicated in Section 11.10.5.1.

Let $\mathcal{F}$ be a foliation on a manifold $M$ satisfying the current conditions, and let $\mathcal{L}$ be a $C^{\infty}$ foliation of dimension one transverse to $\mathcal{F}$. For any saturated open connected subset $U \subset M$, the minimal sets of $\left.\mathcal{F}\right|_{U}$ are called local minimal sets. For every leaf $L \subset U, \bar{L} \cap U$ contains a nonzero finite number of minimal sets of $\left.\mathcal{F}\right|_{U}$.

Any proper leaf is a local minimal set. A nonempty saturated open connected set $U$ is a local minimal set if $\left.\mathcal{F}\right|_{U}$ is minimal, which is called of locally dense type. All other local minimal sets are called of exceptional type, and meet the leaves of $\mathcal{L}$ in a sets homeomorphic to open subsets of the Cantor set. Obviously, minimal sets are local minimal sets.

A minimal set (and each of its leaves) is said to be at level 0 . A local minimal set $X$ (and each of its leaves) is said to be at level $k \in \mathbb{Z}^{+}$if the highest level of any local minimal set in $\bar{X} \backslash X$ is $k-1$. The leaves that are not at finite level are said to be at infinite level. For $k \in \mathbb{N}$, the union $M_{k}$ of leaves at levels $\leq k$ is compact. For every leaf $L$, the set $\bar{L} \cap M_{k}$ is a nonempty finite union of local minimal sets. Every local minimal set $U$ is at finite level. 
If $L$ is at infinite level, then $\bar{L} \cap\left(M_{k} \backslash M_{k-1}\right) \neq \varnothing$ (taking $\left.M_{-1}=\varnothing\right)$. Let $X=\bigcup_{k} \bar{L} \cap M_{k}$ and $Z=\bar{L} \backslash X$. Then $X$ is dense in $\bar{L}, Z$ is an uncountable union of leaves, each leaf in $Z$ is dense in $\bar{L}$, and no leaf of $Z$ has a proper side.

The substructure of a leaf $L$ is the union of leaves $F \subset \bar{L}$ with $\bar{F} \neq \bar{L}$. It is a union of local minimal sets, none of which are of locally dense type. If every leaf in $S(L)$ is proper, then $L$ is said to have a totally proper substructure. If every leaf in $\bar{L}$ is proper, then $L$ is said to be totally proper.

For instance, in Hector's example (Section 11.10.1), the leaves corresponding to orbits in $\Omega$ are at infinite level, the leaf corresponding to every orbit $T_{n}\left(n \in \mathbb{Z}^{+}\right)$ is at level $n$, and the points \pm 1 correspond to one compact leaf at level 0 .

11.10.5.1. Growth of leaves at finite level. Let us recall some results and examples about the growth of leaves at finite level due to Cantwell and Conlon [26] (see also 97,98 ).

With the notation of Section 11.10.5, let $L$ be a leaf of $\mathcal{F}$ of non-exponential growth. Then $L$ has totally proper substructure [26 Corollary 3.4]. If moreover $L$ is semi-proper, then $L$ is totally proper and has exactly polynomial growth of degree equal to its level [26, Corollaries 3.5 and 3.6]. In this case, the transitive compact foliated space $X=\bar{L}$ obviously satisfies the alternative (i) of Theorems 1.2 and 1.6 ( $L$ is its unique dense leaf).

Let $L$ be a non-exponential leaf of $\mathcal{F}$ at finite level $k$. If $L$ does not have a proper side, then $L$ is in a local minimal set $U$ of locally dense type, $\left.\mathcal{F}\right|_{U}$ has trivial holonomy with the leaves mutually diffeomorphic and the same growth type, and $\bar{U} \backslash U$ is a finite union of totally proper leaves whose maximum level is $k-1$ [26. Theorem 3.7].

Cantwell and Conlon described a family $\mathbf{G}$ of growth types, which contains a continuum infinity of distinct quasi-polynomial but non-polynomial types, a continuum of distinct non-exponential but non-quasi-polynomial types, and the exponential growth type. As indicated in Chapter 11, they proved that, for all closed 3 -manifold and $\gamma \in \mathbf{G}$, there is a $C^{\infty}$ foliation $\mathcal{F}$ in $M$ containing a local minimal set $U$ of locally dense type such that $\bar{U} \backslash U$ is a finite union of totally proper leaves, and $\left.\mathcal{F}\right|_{U}$ has trivial holonomy with the leaves mutually diffeomorphic and growth type $\gamma$ [26. Theorem 5.1]. They also observed that the construction can be adapted to produce leaves of polynomial growth of any degree $\geq 3$ in $U$.

In these cases, the diffeomorphisms between the leaves in $U$ is given by a $C^{0}$ foliated ${ }^{10}$ flow, which is non-singular precisely on $U$, and with differentiable restrictions to the leaves. Therefore the leaves in $U$ are also mutually differentiable quasi-isometric. Then $\bar{U}$ is a transitive compact foliated space satisfying the alternatives Theorem 1.2. (ii) and Theorem 1.6r(ii).

More results are proved by Cantwell and Conlon with the same interpretation in terms of Theorems 1.2 and 1.6 [26, Theorems 5.5, 6.2, 6.13 and 7.1].

A similar theory of levels was considered by Lukina $\mathbf{7 8}$ for the graph matchbox manifold $\mathcal{T}\left(F_{n}\right)$. But, concerning growth types of leaves, it is quite different from the case of the results of codimension one; for instance, there is a totally proper leaf at level 1 of exponential growth [78, Theorem 1.10].

\subsection{Open problems}

Consider the notation and general conditions of Chapter 1

\footnotetext{
${ }^{10}$ The flow maps leaves to leaves.
} 
Problem 1. Prove versions of Theorems 1.11 .3 for the relation " $x \sim y$ if and only if $L_{x}$ is differentiably quasi-isometric to $L_{y}$ " on $X$, assuming that $\mathcal{F}$ is differentiable.

Problem 1 should not be difficult: the usual local Reeb stability and ArzelaAscoli theorems should be enough to adapt the proofs of Theorems 1.111 .3 .

Problem 2. Suppose that $\mathcal{F}$ is minimal and residually many leaves have a Cantor space of ends. What can be said about the possible coarse quasi-isometry types of the leaves in $X_{0}$ ? Are they equi-coarsely quasi-isometric one another (the alternative (ii) of Theorem 1.2]? Is it possible to characterize the possible coarsely quasi-isometric types that can be realized in this way? What can be said about the leaves with holonomy? The differentiable version of this problem can be also considered, specially for dimension two.

As indicated in Chapter 1, the version of Problem 2 for 2-ended leaves was solved by Blanc $\mathbf{1 9}$. In the case of a Cantor space of ends, one should try to prove, for the leaves in $X_{0}$, a coarsely quasi-isometric version of Stallings' description of finitely generated groups with a Cantor space of ends, using amalgamated free products or HNN extensions [95] (see also [21, Theorem 8.32]). A measure theoretic version of such a description is given in $[\mathbf{5 0}$, Theorem $\mathrm{D}]$. The case of 1-ended leaves is much more difficult.

Problem 3. Suppose that $(X, \mathcal{F})$ is transitive (or minimal). Consider the following equivalence relations on $X$ :

(a) " $x \sim y$ if and only if $L_{x}$ is coarsely quasi-isometric to $L_{y}$."

(b) " $x \sim y$ if and only if $L_{x}$ has the same growth type as $L_{y}$."

(c) " $x \sim y$ if and only if $L_{x}$ is differentiably quasi-isometric to $L_{y}$ " (assuming that

$\mathcal{F}$ is differentiable).

Assume also that $(X, \mathcal{F})$ satisfies the alternative (ii) of Theorem 1.2 in the case of the relation (a), the alternative (ii) of Theorem 1.6 in the case of the relation (b), and a similar alternative in the case of the relation (c). With the terminology of Section 11.10.2, is any of these relations generically ergodic with respect to the isomorphism relation on countable models? Is there any example where some of them is classifiable by countable models?

As suggested by Hector, Problem 3 is especially interesting in the case of foliations of codimension one, confronting it with their Poincaré-Bedixson theory 64, 23. The techniques from [9] may be useful to address Problem 3 (see Section 11.10.4.

Problem 4. What can be said about the coarse cohomology and other coarse algebraic invariants [84] of the generic leaf of a minimal foliated space?

Problem 5. It is not hard to show that the generic leaf of an exceptional minimal set of a codimension one $C^{2}$-foliation of a compact manifold has either one end or a Cantor set of ends. (This is false in class $C^{1}$.) A famous but unpublished result of Duminy (see Cantwell-Conlon [29 for the statement and proof) states that every semiproper leaf of an exceptional minimal set of a codimension one foliation of class $C^{2}$ has a Cantor set of ends. The natural conjecture is that the generic leaf of an exceptional minimal set of a codimension one $C^{2}$-foliation of a compact manifold has a Cantor set of ends, cf. [24, Remark, page 131]. Cantwell and Conlon have proved this and much more for a large class of exceptional minimal sets [30]. 



\section{Bibliography}

[1] F. Alcalde Cuesta, A. Lozano Rojo, and M. Macho Stadler. Dynamique transverse de la lamination de Ghys-Kenyon. Astérisque, 323:1-16, 2009.

[2] F. Alcalde Cuesta, A. Lozano Rojo, and M. Macho Stadler. Transversely Cantor laminations as inverse limits. Proc. Amer. Math. Soc., 139(7):2615-2630, 2011.

[3] F. Alcalde Cuesta and A. Rechtman. Minimal Følner foliations are amenable. Discrete Contin. Dyn. Syst., 31(3):685-707, 2011.

[4] J.-P. Allouche and J. Shallit. Automatic Sequences. Theory, Applications, Generalizations. Cambridge University Press, Cambridge, 2003.

[5] J.A. Álvarez López and R. Barral Lijó. Limit aperiodic and repetitive colorings of graphs. In preparation.

[6] J.A. Álvarez López and R. Barral Lijó. Bounded geometry and leaves. Math. Nachr., 290(10):1448-1469, 2017.

[7] J.A. Álvarez López, R. Barral Lijó, and A. Candel. A universal Riemannian foliated space. Topology Appl., 198:47-85, 2016.

[8] J.A. Álvarez López and A. Candel. Non-reduction of relations in the Gromov space to Polish actions. Notre Dame J. Form. Log., to appear. Preprint arXiv:1501.02606.

[9] J.A. Álvarez López and A. Candel. On turbulent relations. Fund. Math., to appear. Preprint arXiv:1209.0307.

[10] J.A. Álvarez López and A. Candel. Equicontinuous foliated spaces. Math. Z., 263(4):725$774,2009$.

[11] J.A. Álvarez López and A. Candel. Algebraic characterization of quasi-isometric spaces via the Higson compactification. Topology Appl., 158(13):1679-1694, 2011.

[12] O. Attie and S. Hurder. Manifolds which cannot be leaves of foliations. Topology, 35(2):335$353,1996$.

[13] N. Aubrun, S. Barbieri, and S. Thomassé. Realization of aperiodic subshifts and uniform densities in groups. Groups Geom. Dyn., to appear. Preprint arXiv:1507.03369v2.

[14] J. Auslander. Minimal flows and their extensions, volume 153 of North-Holland Mathematics Studies. North-Holland Publishing Co., Amsterdam, 1988.

[15] H. Becker and A.S. Kechris. The descriptive set theory of Polish group actions, volume 232 of London Mathematical Society Lecture Note Series. Cambridge University Press, Cambridge, 1996.

[16] G. Bell and A. Dranishnikov. Asymptotic dimension in Będlewo. Topology Proc., 38:209-236, 2011.

[17] I. Biringer and M. Abert. Unimodular measures on the space of all Riemannian manifolds. Preprint arXiv:1606.03360v3.

[18] E. Blanc. Propriétés génériques des laminations. PhD thesis, Université de Claude BernardLyon 1, Lyon, 2001.

[19] E. Blanc. Laminations minimales résiduellement à 2 bouts. Comment. Math. Helv., $78(4): 845-864,2003$.

[20] J. Block and S. Weinberger. Aperiodic tilings, positive scalar curvature and amenability of spaces. J. Amer. Math. Soc., 5(4):907-918, 1992.

[21] M.R. Bridson and A. Haefliger. Metric spaces of non-positive curvature, volume 319 of Grundlehren der Mathematischen Wissenschaften [Fundamental Principles of Mathematical Sciences]. Springer-Verlag, Berlin, 1999.

[22] A. Candel. The harmonic measures of Lucy Garnett. Adv. Math., 176(2):187-247, 2003.

[23] A. Candel and L. Conlon. Foliations. I, volume 23 of Graduate Studies in Mathematics. American Mathematical Society, Providence, RI, 2000. 
[24] A. Candel and L. Conlon. Foliations. II, volume 60 of Graduate Studies in Mathematics. American Mathematical Society, Providence, RI, 2003.

[25] J. Cantwell and L. Conlon. Poincaré-Bendixson theory for leaves of codimension one. Trans. Amer. Math. Soc., 265(1):181-209, 1981.

[26] J. Cantwell and L. Conlon. Nonexponential leaves at finite level. Trans. Amer. Math. Soc., 269(2):637-661, 1982.

[27] J. Cantwell and L. Conlon. Every surface is a leaf. Topology, 26(3):265-285, 1987.

[28] J. Cantwell and L. Conlon. Generic leaves. Comment. Math. Helv., 73(2):306-336, 1998.

[29] J. Cantwell and L. Conlon. Endsets of exceptional leaves; a theorem of G. Duminy. In Foliations: geometry and dynamics (Warsaw, 2000), pages 225-261. World Sci. Publ., River Edge, NJ, 2002.

[30] John Cantwell and Lawrence Conlon. Foliations and subshifts. Tohoku Math. J. (2), 40(2):165-187, 1988.

[31] D.M. Cass. Minimal leaves in foliations. Trans. Amer. Math. Soc., 287(1):201-213, 1985.

[32] B. Chaluleau and C. Pittet. Exemples de variétés riemanniennes homogènes qui ne sont pas quasi isométriques à un groupe de type fini. C. R. Acad. Sci. Paris Sér. I Math., 332(7):593$595,2001$.

[33] J. Cheeger. Finiteness theorems for Riemannian manifolds. Amer. J. Math., 92:61-74, 1970.

[34] A. Clark and S. Hurder. Homogeneous matchbox manifolds. Trans. Amer. Math. Soc., 365:3151-3191, 2013.

[35] M. Coornaert and A. Papadopoulos. Symbolic dynamics and hyperbolic groups, volume 1539 of Lecture Notes in Mathematics. Springer-Verlag, Berlin, 1993.

[36] A. Denjoy. Sur les courbes définies par les équations différentielles à la surface du tore. $J$. Math. Pures Appl. (9), 11:333-375, 1932.

[37] A. Dranishnikov. Asymptotic topology. Russian Math. Surveys, 55(6):1085-1129, 2000.

[38] A. Dranishnikov, J. Keesling, and V. Uspenskij. On the Higson corona of uniformly contractible spaces. Topology, 37(4):791-803, 1998.

[39] J. Dugundji. Topology. Allyn and Bacon Inc., Boston, Mass., 1978. Reprinting of the 1966 original, Allyn and Bacon Series in Advanced Mathematics.

[40] P.E. Ehrlich. Continuity properties of the injectivity radius function. Compositio Math., 29:151-178, 1974.

[41] J. Eichhorn. The boundedness of connection coefficients and their derivatives. Math. Nachr., 152:145-158, 1991.

[42] D.B.A. Epstein, K.C. Millett, and D. Tischler. Leaves without holonomy. J. London Math. Soc. (2), 16(3):548-552, 1977.

[43] A. Eskin, D. Fisher, and K. Whyte. Coarse differentiation of quasi-isometries I: Spaces not quasi-isometric to Cayley graphs. Ann. of Math. (2), 176(1):221-260, 2012.

[44] D. Feldman. A weakly homogeneous rigid space. Topology Appl., 38(1):97-100, 1991.

[45] J. Feldman and C.C. Moore. Ergodic equivalence relations, cohomology, and von Neumann algebras. I. Trans. Amer. Math. Soc., 234(2):289-324, 1977.

[46] S. Gao, S. Jackson, and B. Seward. A coloring property for countable groups. Math. Proc. Cambridge Philos. Soc., 147(3):579-592, 2009.

[47] L. Garnett. Foliations, the ergodic theorem and Brownian motion. J. Funct. Anal., 51(3):285-311, 1983

[48] R. Geoghegan. Topological methods in group theory. Springer, New York, 2008.

[49] É. Ghys. Une variété qui n'est pas une feuille. Topology, 24(1):67-73, 1985.

[50] É. Ghys. Topologie des feuilles génériques. Ann. of Math. (2), 141(2):387-422, 1995.

[51] É. Ghys. Laminations par surfaces de Riemann. In Dynamique et géométrie complexes (Lyon, 1997), volume 8, pages 49-95, 1999.

[52] C. Godbillon. Feuilletages, volume 98 of Progress in Mathematics. Birkhäuser Verlag, Basel, 1991. Études géométriques. With a preface by G. Reeb.

[53] W.H. Gottschalk and G.A. Hedlund. Topological dynamics, volume 36 of American Mathematical Society Colloquium Publications. American Mathematical Society, Providence, R.I., 1955.

[54] M. Gromov. Groups of polynomial growth and expanding maps. Appendix by Jacques Tits. Inst. Hautes Études Sci. Publ. Math., 53:53-73, 1981. 
[55] M. Gromov. Asymptotic invariants of infinite groups. In Geometric group theory, Vol. 2 (Sussex, 1991), volume 182 of London Math. Soc. Lecture Note Ser., pages 1-295. Cambridge Univ. Press, Cambridge, 1993.

[56] M. Gromov. Metric structures for Riemannian and non-Riemannian spaces, volume 152 of Progress in Mathematics. Birkhäuser Boston Inc., Boston, MA, 1999. Based on the 1981 French original [MR0682063], With appendices by M. Katz, P. Pansu and S. Semmes, Translated from the French by Sean Michael Bates.

[57] A. Haefliger. Pseudogroups of local isometries. In Differential geometry (Santiago de Compostela, 1984), volume 131 of Res. Notes in Math., pages 174-197. Pitman, Boston, MA, 1985.

[58] A. Haefliger. Leaf closures in Riemannian foliations. In A fête of topology, pages 3-32. Academic Press, Boston, MA, 1988.

[59] A. Haefliger. Foliations and compactly generated pseudogroups. In Foliations: Geometry and Dynamics, Warsaw 2000, pages 275-296, River Edge, NJ, 2002. World Sci. Publi.

[60] G. Hector. Quelques exemples de feuilletages espèces rares. Ann. Inst. Fourier (Grenoble), 26(1):239-264, 1976.

[61] G. Hector. Feuilletages en cylindres. In Geometry and topology (Proc. III Latin Amer. School of Math., Inst. Mat. Pura Aplicada CNPq, Rio de Janeiro, 1976), pages 252-270. Lecture Notes in Math., Vol. 597. Springer, Berlin, 1977.

[62] G. Hector. Leaves whose growth is neither exponential nor polynomial. Topology, 16(4):451$459,1977$.

[63] G. Hector and U. Hirsch. Introduction to the geometry of foliations. Part A, volume 1 of Aspects of Mathematics. Friedr. Vieweg \& Sohn, Braunschweig, second edition, 1986. Foliations on compact surfaces, fundamentals for arbitrary codimension, and holonomy.

[64] G. Hector and U. Hirsch. Introduction to the geometry of foliations. Part B. Aspects of Mathematics, E3. Friedr. Vieweg \& Sohn, Braunschweig, second edition, 1987. Foliations of codimension one.

[65] N. Higson and J. Roe. Analytic K-homology. Oxford Mathematical Monographs. Oxford University Press, Oxford, 2000. Oxford Science Publications.

[66] G. Hjorth. Classification and orbit equivalence relations, volume 75 of Mathematical Surveys and Monographs. American Mathematical Society, Providence, RI, 2000.

[67] G. Hjorth. A dichotomy theorem for turbulence. J. Symbolic Logic, 67(4):1520-1540, 2002.

[68] S. Hurder. Coarse geometry of foliations. In T. Mizutani; K. Masuda; S. Matsumoto; T. Inaba; T. Tsuboi and Y. Mitsumatsu, editors, Geometric study of foliations. Proceedings of the international symposium/workshop, Tokyo, Japan, November 15-19 and 22-26, 1993, pages 35-96, River Edge, NJ, 1994. World Scientific.

[69] T. Inaba, T. Nishimori, M. Takamura, and N. Tsuchiya. Open manifolds which are nonrealizable as leaves. Kodai Math. J., 8(1):112-119, 1985.

[70] V.A. Kaimanovich. Equivalence relations with amenable leaves need not be amenable. In Topology, ergodic theory, real algebraic geometry. Rokhlin's memorial, volume 202 of Amer. Math. Soc. Transl. Ser. 2, pages 151-166. Amer. Math. Soc., Providence, RI, 2001.

[71] A.S. Kechris. Classical descriptive set theory, volume 156 of Graduate Texts in Mathematics. Springer-Verlag, New York, 1995.

[72] A.S. Kechris and B.D. Miller. Topics in orbit equivalence, volume 1852 of Lecture Notes in Mathematics. Springer-Verlag, Berlin, 2004.

[73] P. Lessa. Reeb stability and the Gromov-Hausdorff limits of leaves in compact foliations. Asian J. Math., 19(3):433-464, 2015.

[74] Á. Lozano Rojo. Foliated spaces defined by graphs. Rev. Semin. Iberoam. Mat., 3(4):21-38, 2007.

[75] Á. Lozano Rojo. Dinamica transversa de laminaciones definidas por grafos repetitivos. $\mathrm{PhD}$ thesis, UPV-EHU, 2008.

[76] Á. Lozano Rojo. Codimension zero laminations are inverse limits. Topology Appl., 160(2):341-349, 2013.

[77] Á. Lozano Rojo and O. Lukina. Suspensions of Bernoulli shifts. Dyn. Syst., 28(4):551-566, 2013.

[78] O. Lukina. Hierarchy of graph matchbox manifolds. Topology Appl., 159(16):3461-3485, 2012. 
[79] C.C. Moore and C. Schochet. Global analysis on foliated spaces, volume 9 of Mathematical Sciences Research Institute Publications. Springer-Verlag, New York, 1988. With appendices by S. Hurder, Moore, Schochet and Robert J. Zimmer.

[80] P. Perrin and J.-É. Pin. Infinite words. Automata, semigroups, logic and games, volume 141 of Pure and Applied Mathematics. Elsevier/Academic Press, Amsterdam, 2004.

[81] P. Petersen. Riemannian geometry, volume 171 of Graduate Texts in Mathematics. SpringerVerlag, New York, 1998.

[82] N. Pytheas Fogg. Substitutions in dynamics, arithmetics and combinatorics, volume 1794 of Lecture Notes in Math. Springer, Berlin, 2002. Edited by V. Berthé, S. Ferenczi, C. Mauduit and A. Siegel.

[83] J. Roe. An index theorem on open manifolds. I. J. Differential Geom., 27(1):87-113, 1988.

[84] J. Roe. Coarse cohomology and index theory on complete Riemannian manifolds. Mem. Amer. Math. Soc., 104(497), 1993.

[85] J. Roe. Index theory, coarse geometry, and topology of manifolds, volume 90 of CBMS Regional Conference Series in Mathematics. published for the Conference Board of the Mathematical Sciences, Washington, DC, by the American Mathematical Society, Providence, RI, 1996.

[86] J. Roe. Lectures on coarse geometry, volume 31 of University Lecture Series. American Mathematical Society, Providence, RI, 2003.

[87] T. Sakai. On continuity of injectivity radius function. Math. J. Okayama Univ., 25(1):91-97, 1983.

[88] T. Schick. Analysis on D-Manifolds of Bounded Geometry, Hodge-De Rham Isomorphism and $L^{2}$-Index Theorem. PhD thesis, Johannes Gutenberg Universität Mainz, Mainz, 1996.

[89] T. Schick. Manifolds with boundary and of bounded geometry. Math. Nachr., 223:103-120, 2001.

[90] P.A. Schweitzer. Surfaces not quasi-isometric to leaves of foliations of compact 3-manifolds. In Analysis and geometry in foliated manifolds, Proceedings of the VII International Colloquium on Differential Geometry, Santiago de Compostela, Spain, July 26-30, 1994, pages 223-238. World Sci. Publ., Singapore, 1995.

[91] P.A. Schweitzer. Riemannian manifolds not quasi-isometric to leaves in codimension one foliations. Ann. Inst. Fourier (Grenoble), 61(4):1599-1631, 2011.

[92] P.A. Schweitzer and F.S. Souza. Manifolds that are not leaves of codimension one foliations. Int. J. Math., 24(14):14 pp., 2013.

[93] M.A. Shubin. Spectral theory of elliptic operators on noncompact manifolds. Astérisque, 207:35-108, 1992. Méthodes semi-classiques, Vol. 1 (Nantes, 1991)

[94] F.S. Souza. Non-leaves of some foliations. PhD thesis, Pontifícia Universidade Católica do Rio de Janeiro, 2011.

[95] J.R. Stallings. On torsion-free groups with infinitely many ends. Ann. of Math. (2), 88:312$334,1968$.

[96] D. Sullivan. Inside and outside manifolds. In Proceedings of the International Congress of Mathematicians (Vancouver, B. C., 1974), volume 1, pages 201-207, Montreal, Quebec, 1975. Canad. Math. Congress.

[97] N Tsuchiya. Growth and depth of leaves. J. Fac. Sci. Univ. Tokyo Sect. IA Math., 26(3):473$500,1979$.

[98] N. Tsuchiya. Leaves with non-exact polynomial growth. Tôhoku Math. J. (2), 32(1):71-77, 1980.

[99] G. Yu. The Novikov conjecture for groups with finite asymptotic dimension. Ann. of Math. (2), 147(2):325-355, 1998.

[100] A. Zeghib. An example of a 2-dimensional no leaf. In Geometric study of foliations (Tokyo, 1993), pages 475-477. World Sci. Publ., River Edge, NJ, 1994. 